\title{
Two-loop RGE of a general renormalizable Yang-Mills theory in a renormalization scheme with an explicit UV cutoff
}

\author{
Piotr H. Chankowski, ${ }^{a}$ Adrian Lewandowski ${ }^{b, a}$ and Krzysztof A. Meissner ${ }^{a}$ \\ ${ }^{a}$ Institute of Theoretical Physics, Faculty of Physics, University of Warsaw, \\ Pasteura 5, 02-093 Warsaw, Poland \\ ${ }^{b}$ Max-Planck-Institut für Gravitationsphysik (Albert-Einstein-Institut), \\ Mühlenberg 1, D-14476 Potsdam, Germany \\ E-mail: piotr.chankowski@fuw.edu.pl, adrian.lewandowski@aei.mpg.de, \\ krzysztof.meissner@fuw.edu.pl
}

ABSTRACT: We perform a systematic one-loop renormalization of a general renormalizable Yang-Mills theory coupled to scalars and fermions using a regularization scheme with a smooth momentum cutoff $\Lambda$ (implemented through an exponential damping factor). We construct the necessary finite counterterms restoring the BRST invariance of the effective action by analyzing the relevant Slavnov-Taylor identities. We find the relation between the renormalized parameters in our scheme and in the conventional $\overline{\mathrm{MS}}$ scheme which allow us to obtain the explicit two-loop renormalization group equations in our scheme from the known two-loop ones in the $\overline{\mathrm{MS}}$ scheme. We calculate in our scheme the divergences of two-loop vacuum graphs in the presence of a constant scalar background field which allow us to rederive the two-loop beta functions for parameters of the scalar potential. We also prove that consistent application of the proposed regularization leads to counterterms which, together with the original action, combine to a bare action expressed in terms of bare parameters. This, together with treating $\Lambda$ as an intrinsic scale of a hypothetical underlying finite theory of all interactions, offers a possibility of an unconventional solution to the hierarchy problem if no intermediate scales between the electroweak scale and the Planck scale exist.

KEYwords: BRST Quantization, Renormalization Regularization and Renormalons, Renormalization Group, Anomalies in Field and String Theories

ArXIV EPrint: 1608.02270 


\section{Contents}

1 Introduction $\quad 2$

2 Lagrangian and conventions $\quad 4$

$\begin{array}{lll}3 & \text { The UV regularization } & 7\end{array}$

4 The subtraction procedure $\quad 10$

5 Determination of the BRST symmetry restoring counterterms $\quad 16$

$\begin{array}{ll}5.1 \text { Identity involving the }\langle\psi \psi A\rangle \text { function } & 18\end{array}$

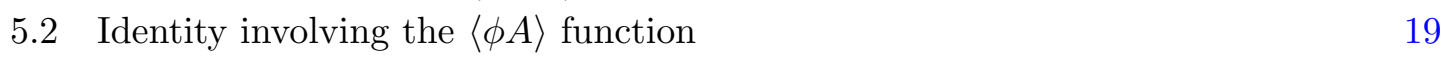

5.3 Identity involving the $\langle A A\rangle$ function 20

5.4 Identity involving the $\langle\phi \phi A\rangle$ function 21

5.5 Identities involving the $\langle\phi A A\rangle$ and $\langle\phi \phi A A\rangle$ functions 22

5.6 Identity involving the $\langle A A A\rangle$ function $\quad 22$

5.7 Identity involving the $\langle A A A A\rangle$ function 24

6 Relation between $\Lambda$ - $\overline{\mathrm{MS}}$ and DimReg- $\overline{\mathrm{MS}} \quad 26$

$\begin{array}{llr}7 & \text { Renormalization group equations } & 30\end{array}$

8 The "bare" scalar potential $\quad 38$

9 Bare parameters and the hierarchy problem $\quad 45$

10 Conclusions $\quad 51$

A Auxiliary conditions in the subtraction procedure $\quad \mathbf{5 2}$

$\begin{array}{lll}\text { A.1 Auxiliary conditions } & 52\end{array}$

A.2 Completion of the inductive step $\quad 55$

$\begin{array}{ll}\text { B One-loop diagrams } & 56\end{array}$

$\begin{array}{ll}\text { C Chiral anomaly } & 65\end{array}$

$\begin{array}{ll}\text { D RGE } & 68\end{array}$

$\begin{array}{lll}\text { E DimReg- } \overline{M S} \text { beta function } \check{\boldsymbol{\beta}} & 70\end{array}$

$\begin{array}{lll}\text { F } & \text { The Standard Model case } & 72\end{array}$

$\begin{array}{ll}\text { G Basic 2-loop integrals } & 75\end{array}$ 


\section{Introduction}

Renormalization is in quantum field theory a standard procedure. It not only renders calculated quantities finite but also, when the freedom in implementing it is judiciously exploited, allows to analyze the behavior of the computed Green's functions and observables when the characteristic energy scale changes. The first step in this procedure is usually the introduction of an ultraviolet (UV) regularization (an UV cutoff). The second one is performing appropriate subtractions (usually interpreted as an effect of taking into account contributions of suitable counterterms) after which the UV cutoff can be removed leaving finite amplitudes. The freedom in the subtractions (in the choice of the renormalization scheme) can be used either to directly parametrize the computed quantities in terms of a selected set of measured observables or to introduce an arbitrary scale $\mu$ and parametrize the theory predictions with a set of finite, $\mu$ dependent parameters (hybrid schemes are also possible). The requirement that physical results be independent of $\mu$ gives then rise to the renormalization group (RG) which in turn allows for the mentioned possibility of analyzing the dependence of predictions on the energy scale. The most frequently used scheme of this second type is the (modified) minimal subtraction $\overline{\mathrm{MS}}$ applied to dimensionally regularized amplitudes which automatically introduces an arbitrary scale $\mu$. Renormalization of Yang Mills (YM) theories is usually studied using this scheme [1] the main reason being that the dimensional regularization (DimReg), unlike other more physical UV cutoffs, automatically preserves (in theories like QCD, without fermions in chiral representations) the BRST symmetry. This greatly facilitates the construction of the finite (renormalized) effective action which must be BRST-symmetric. This property of the effective action is indispensable to ensure decoupling of unphysical degrees of freedom (Faddeev-Popov ghosts and antighost, scalar components of vector bosons, would-be Goldstone modes in the case of broken gauge symmetries or longitudinal vector bosons of unbroken gauge symmetries) and unitarity of the $S$-matrix in the physical subspace of the full (pseudo-)Hilbert space.

However, DimReg, while being elegant and convenient as a technical tool, has some rather unphysical features. In particular it sets (by definition) to zero the whole class of contributions to the effective action which are due to real fluctuations of quantum fields but which happen to be quadratically divergent with an explicit momentum ultra-violet cutoff $\Lambda$ (however introduced). It is also hard to interpret physically the departure from the integer dimension of the space-time. These drawbacks do not, of course, create any problem for practical calculations aiming at expressing low energy observables in terms of a selected set of other low energy observables (or in terms of another set of finite parameters), in which, after performing subtractions, the cutoff is completely removed, but certainly obscure understanding of the problem of stability of the electroweak scale $G_{\mathrm{F}}^{-1 / 2}$ versus the Planck scale $M_{\mathrm{Pl}}$.

In this paper we would like to adopt a more fundamental point of view on renormalization (close in spirit to the one taken in applications of field theory to statistical physics problems), proposed in [2] (see also [3]), which we motivate (in section 9) by its possible connection with the hierarchy problem. This view precludes using unphysical regularizations like DimReg and requires treating the momentum space cutoff $\Lambda$ as a bona fide 
physical scale which in our approach is viewed as an intrinsic scale of a fundamental theory of physics at the Planck scale (and, therefore, the limit $\Lambda \rightarrow \infty$ is not taken). This leads us to study renormalization of a general YM theory coupled to scalars and fermions using an explicit momentum cutoff $\Lambda$. The use of the momentum cutoff as the regulator in YM theories immediately brings in the problem that the regulated Green's functions do not satisfy the requisite Slavnov-Taylor (ST) identities following from the BRST invariance. This calls for a special form of subtractions which must restore these identities. ${ }^{1}$ We recall in this connection the general procedure for achieving this, which is based on the Quantum Action Principle (QAP) [6-9], and implement it in the explicit one-loop calculations. We point out, however, that strict BRST invariance is recovered with the help of this procedure only in the limit of infinite $\Lambda$; for finite $\Lambda$ the ST identities remain broken by terms suppressed by inverse powers of $\Lambda^{2}$ and one has to assume that other effects of the underlying fundamental theory act so that effectively all potential problems associated with this breaking are cured.

To our knowledge, renormalization of YM theories in the regularization based on an explicit momentum cutoff has never been studied systematically. In this paper we provide the necessary technical tools for developing the approach sketched in [2] and perform the systematic one-loop renormalization of a general renormalizable YM theory coupled to scalars and fermions in arbitrary (but non-anomalous) representations using the explicit UV cutoff proposed there. The paper is organized as follows. In section 2 we explain our notation and conventions and recall basic facts concerning the BRST symmetry. In section 3 we specify our choice of the UV cutoff which introduces a scale $\Lambda$ and present some technicalities concerning practical evaluation of Feynman diagrams. Section 4 is devoted to the general procedure of making subtractions restoring the BRST invariance. Here we also specify our renormalization scheme which, similarly as the ordinary $\overline{\mathrm{MS}}$ scheme, introduces an arbitrary scale $\mu$. Explicit determination of the one-loop counterterms and of the relation between renormalized parameters in our scheme and in DimReg occupy sections 5 and 6 . The results of section 6 can be also read as an extension to the most general case of the results of [10], namely as a proof of equivalence at one-loop of the $\overline{\mathrm{MS}}$ scheme with anticommuting $\gamma^{5}$ matrix with a fully consistent renormalization prescription. In section 7 we introduce the RG equation. We argue that the standard reasoning justifying it is not directly applicable to regularizations which break the BRST invariance and, therefore, $\mu$ independence of the results requires a separate proof (which we offer). The renormalization group allows for the use the concept of bare action whose structure in the case of our regularization is elucidated. In the same section using the relation of our subtraction scheme with the standard $\overline{\mathrm{MS}}$ scheme we derive two-loop renormalization group equations satisfied by parameters (couplings and mass parameters) of a general YM theory. In section 8 we apply our regularization prescription to the two-loop computation of the scalar fields effective potential focusing, however, only on its divergences. We determine in this way the two-loop coefficient proportional to $\Lambda^{2}$ of the counterterm to the effective potential which turns out to be different than that found using the dimensional reduction (DimRed) [11, 12] which has been recently

\footnotetext{
${ }^{1}$ An alternative approach is to device a cutoff regularization which preserves an appropriately modified version of the BRST symmetry $[4,5]$.
} 
reproduced in [13] using a cutoff regularization superficially similar to ours. We explain the difference between our result and that in [13]. We also determine the one-loop coefficient of $\Lambda^{2}$ in the counterterm to vector boson masses squared which is not present in DimReg (or DimRed) but is unavoidable in the regularization by a physical momentum cutoff.

The possibility to formulate the theory in terms of the bare action and treating the introduced momentum cutoff scale $\Lambda$ as a physical (finite) scale allows to discuss the hierarchy problem and to propose its possible solution along the lines of ref. [2]. In section 9 we recall the basic idea of this solution (which owing to the results presented in this paper gain more solid foundations) and use the derived two-loop RG equations and the coefficient of $\Lambda^{2}$ divergence of the effective potential to discuss (non) viability of this solution in the SM. Section 10 contains our conclusions.

\section{Lagrangian and conventions}

As the starting point of our approach we consider a general renormalizable Yang-Mills theory with the gauge group which is a direct product of an arbitrary number of compact simple Lie groups and U(1) groups coupled to scalar and fermionic fields in arbitrary representations of the gauge group. We work with real scalars $\phi^{i}$ and represent all fermionic fields as four-component Majorana spinors $\psi^{a}$ built out of fundamental two-component Weyl spinors. ${ }^{2}$ It is also convenient to consider the theory in the presence of an arbitrary constant scalar background $\varphi^{i}$ which can eventually be identified with the vacuum expectation value (VEV) of "the symmetric phase" field $\Phi^{i}=\phi^{i}+\varphi^{i}$. (This identification, however, will not be used in what follows). Thus the classical gauge-invariant action $I_{0}^{G I}$ (prior to regularization) is given by the integral of the Lagrangian density

$$
\begin{aligned}
\mathcal{L}_{0}^{G I}= & -\frac{1}{4} \delta_{\alpha \beta} F_{\mu \nu}^{\alpha} F^{\beta \mu \nu}+\frac{1}{2} \delta_{i j}\left(D_{\mu} \phi\right)^{i}\left(D^{\mu} \phi\right)^{j}-\mathcal{V}(\phi+\varphi) \\
& +\frac{1}{2} \bar{\psi}^{a}\left\{\delta_{a b} i\left(\gamma^{\mu} D_{\mu} \psi\right)^{b}-\left[\widehat{\mathcal{M}}_{F}(\phi+\varphi)\right]_{a b} \psi^{b}\right\} .
\end{aligned}
$$

The potential $\mathcal{V}(\Phi)$ is a fourth order polynomial. It is parametrized by the following coupling constants and mass parameters:

$$
\lambda_{i j k l}=\mathcal{V}_{i j k l}^{(4)}(\varphi), \quad\left[\rho_{i}\right]_{j k}=\mathcal{V}_{i j k}^{\prime \prime \prime}(\varphi), \quad m_{S i j}^{2}=\mathcal{M}_{S}^{2}(\varphi)_{i j}=\mathcal{V}_{i j}^{\prime \prime}(\varphi), \quad \mathcal{V}_{i}^{\prime}(\varphi) \neq 0
$$

which, with the exception of $\lambda_{i j k l}$, are $\varphi$-dependent. The generalized fermion mass matrix, which is a first order polynomial in $\Phi^{i}$, includes also the Yukawa couplings

$$
\widehat{\mathcal{M}}_{F}(\Phi)=\widehat{\mathcal{M}}_{F}(0)+y_{i} \Phi^{i} .
$$

Different kinds of indices are lowered/raised with the aid of the appropriate metrics: $\delta_{i j}$, $\delta_{\alpha \beta}, \delta_{a b}$ for internal indices and $\eta_{\mu \nu}=\operatorname{diag}(+1,-1,-1,-1)$ for Lorentz indices.

The explicit form of $F_{\mu \nu}^{\alpha}$ is

$$
F_{\mu \nu}^{\alpha}=\partial_{\mu} A_{\nu}^{\alpha}-\partial_{\nu} A_{\mu}^{\alpha}+e^{\alpha}{ }_{\beta \gamma} A_{\mu}^{\beta} A_{\nu}^{\gamma}
$$

\footnotetext{
${ }^{2}$ Although calculations with the Majorana fields involve the charge conjugation matrix $C$ defined by the relation $\bar{\psi}=\psi^{\mathrm{T}} C$, they are more convenient as they lead to a smaller number of diagrams.
} 
and the covariant derivatives read

$$
D_{\mu} \phi=\partial_{\mu} \phi+A_{\mu}^{\alpha}\left[\mathcal{T}_{\alpha}(\phi+\varphi)+\bar{P}_{\alpha}\right], \quad D_{\mu} \psi=\partial_{\mu} \psi+A_{\mu}^{\alpha} t_{\alpha} \psi
$$

$\mathcal{T}_{\alpha}$ are real antisymmetric generators of the gauge group in the representation formed by the scalars $\phi^{i}$; they satisfy the commutation relations $\left[\mathcal{T}_{\alpha}, \mathcal{T}_{\beta}\right]=\mathcal{T}_{\gamma} e^{\gamma}{ }_{\alpha \beta}$ with the real structure constants $e_{\alpha \beta}^{\gamma}$. Obviously, $e_{\alpha \beta}^{\gamma}$, which themselves are matrix elements of the generators $e_{\alpha}$ in the adjoint representation $\left(\left[e_{\alpha}\right]_{\beta}^{\gamma}=e_{\alpha \beta}^{\gamma}\right.$ ), are, similarly as $\mathcal{T}_{\alpha}$ and $t_{\alpha}$ (and $\mathfrak{f}_{\alpha}$ - see below), proportional to the gauge coupling constants. We work in a natural basis of the gauge Lie algebra, so that the indices $\alpha$ split into Abelian ones $\left(\alpha_{A}\right)$ and semisimple ones $\left(\alpha_{S}\right)$. Coefficients $\bar{P}_{\alpha}$ obeying $\mathcal{T}_{\beta} \bar{P}_{\alpha}=0$ must vanish for non-Abelian indices $\alpha=\alpha_{S}$. If $\bar{P}_{\alpha_{A}} \neq 0$ for some Abelian indices $\alpha_{A}$, Stueckelberg fields are present (see e.g. [14] and references therein) among components of the scalar fields $\phi$ as explained in appendix A.1. In the generators $t_{\alpha}$, similarly as in the generalized fermion mass matrix (2.3), the chiral projectors $P_{L, R}=\frac{1}{2}\left(1 \mp \gamma^{5}\right)$ are included:

$$
t_{\alpha}=\mathfrak{f}_{\alpha} P_{L}+\mathfrak{f}_{\alpha}^{*} P_{R}, \quad \widehat{\mathcal{M}}_{F}(\Phi)=\mathcal{M}_{F}(\Phi) P_{L}+\mathcal{M}_{F}^{*}(\Phi) P_{R},
$$

(likewise $y_{i} \equiv Y_{i} P_{L}+Y_{i}^{*} P_{R}$ ). Here $\mathfrak{f}_{\alpha}$ are ordinary antihermitian matrix generators (satisfying the relation $\left[\mathfrak{f}_{\alpha}, \mathfrak{f}_{\beta}\right]=\mathfrak{f}_{\gamma} e_{\alpha \beta}^{\gamma}$ ) of the gauge group representation realized by the Weyl fields. The background-dependent mass matrix $m_{F}$ of the Weyl fermions has the structure

$$
m_{F} \equiv \mathcal{M}_{F}(\varphi)=\mathcal{M}_{F}(0)+Y_{i} \varphi^{i},
$$

We also write $\widehat{m}_{F} \equiv \widehat{\mathcal{M}}_{F}(\varphi)$ for its Majorana counterpart. The mass matrix of the vector bosons is given by

$$
\left[m_{V}^{2}\right]_{\alpha \beta}=\left[\mathcal{M}_{V}^{2}(\varphi)\right]_{\alpha \beta} \equiv-\frac{1}{2} \varphi^{\mathrm{T}}\left\{\mathcal{T}_{\alpha}, \mathcal{T}_{\beta}\right\} \varphi+\bar{P}_{\alpha}^{\mathrm{T}} \bar{P}_{\beta}
$$

If Stueckelberg fields are absent, $m_{V}^{2}$ vanishes unless the background $\varphi$ has some nonzero components breaking (at least partly) the gauge group. Gauge invariance of $\mathcal{L}_{0}^{G I}$ implies also various important relations between parameters, like e.g.

$$
\left(m_{S}^{2} \mathcal{T}_{\alpha} \varphi\right)_{k}=-\mathcal{V}_{l}^{\prime}(\varphi)\left[\mathcal{T}_{\alpha}\right]_{k}^{l}=\left(\mathcal{T}_{\alpha} \mathcal{V}^{\prime}(\varphi)\right)_{k}
$$

To generate Green's functions of the quantum theory, the classical action $I_{0}^{G I}$ must be supplemented with a gauge fixing term and with the ghost fields action. The structure of divergences arising in the perturbative expansion can be then controlled by working with the BRST invariant tree-level action

$$
I_{0}=I_{0}^{G I}+I_{0}^{\text {Rest }}=\int \mathrm{d}^{4} x\left(\mathcal{L}_{0}^{G I}+\mathcal{L}_{0}^{\text {Rest }}\right),
$$

where $\mathcal{L}_{0}^{\text {Rest }}$ depends on the Nakanishi-Lautrup fields $h_{\beta}$, the ghost fields $\omega^{\alpha}$ and $\bar{\omega}_{\alpha}$ and the so-called antifields $K_{i}, \bar{K}_{a}, K_{\alpha}^{\mu}$ and $L_{\alpha}$ :

$$
\mathcal{L}_{0}^{\text {Rest }}=s\left(\bar{\omega}_{\alpha} \mathfrak{F}^{\alpha}+\frac{1}{2} \bar{\omega}_{\alpha} \xi^{\alpha \beta} h_{\beta}\right)+L_{\alpha} s\left(\omega^{\alpha}\right)+K_{i} s\left(\phi^{i}\right)+\bar{K}_{a} s\left(\psi^{a}\right)+K_{\alpha}^{\mu} s\left(A_{\mu}^{\alpha}\right) .
$$


Here $\xi^{\alpha \beta}$ are arbitrary gauge fixing parameters. In what follows we will work in the Landau gauge

$$
\mathfrak{F}^{\alpha} \equiv-\partial_{\mu} A^{\alpha \mu}, \quad \quad \xi^{\alpha \beta} \equiv 0,
$$

which leads to some simplifications due to the presence in this gauge of additional symmetries of $I_{0}^{\text {Rest }}$ (see appendix A.1).

The action on fields of the BRST "differential" $s(\cdot)$ is given by [15]

$$
\begin{aligned}
& s\left(\phi^{i}\right)=\omega^{\alpha}\left[\mathcal{T}_{\alpha}(\phi+\varphi)+\bar{P}_{\alpha}\right]^{i}, \quad s\left(\psi^{a}\right)=\omega^{\alpha}\left(t_{\alpha} \psi\right)^{a}, \quad s\left(A_{\mu}^{\gamma}\right)=-\partial_{\mu} \omega^{\gamma}+e^{\gamma}{ }_{\alpha \beta} \omega^{\alpha} A_{\mu}^{\beta}, \\
& s\left(\omega^{\alpha}\right)=\frac{1}{2} e_{\beta \gamma}^{\alpha} \omega^{\beta} \omega^{\gamma}, \quad s\left(\bar{\omega}_{\alpha}\right)=h_{\alpha}, \quad s\left(h_{\alpha}\right)=0 .
\end{aligned}
$$

The antifields $K_{i}, \bar{K}_{a}, K_{\alpha}^{\mu}$ and $L_{\alpha}$, treated as external sources, control the renormalization of the composite operators $s\left(\phi^{i}\right), s\left(\psi^{a}\right), s\left(A_{\mu}^{\alpha}\right)$ and $s\left(\omega^{\alpha}\right)$. Setting $s\left(K_{i}\right)=s\left(\bar{K}_{a}\right)=$ $s\left(K_{\alpha}^{\mu}\right)=s\left(L_{\alpha}\right)=0$ makes the action $I_{0}^{\text {Rest }}$ a BRST-exact functional: $I_{0}^{\text {Rest }}=s(W)$. Nilpotency $s^{2}=0$ of the $s(\cdot)$ operation ensures then the BRST invariance of the complete action $(2.9): s\left(I_{0}\right)=0$.

In writing identities expressing the BRST invariance of the effective action we will work in the momentum space representing fields by their Fourier images according to the formulae

$$
A_{\mu}^{\alpha}(x)=\int \mathrm{d}^{4} l e^{-i l x} \tilde{A}_{\mu}^{\alpha}(l), \quad \frac{\delta}{\delta A_{\mu}^{\alpha}(x)}=\int \frac{\mathrm{d}^{4} l}{(2 \pi)^{4}} e^{i l x} \frac{\delta}{\delta \tilde{A}_{\mu}^{\alpha}(l)} .
$$

Momentum space one-particle irreducible (1PI) Green's functions are then given by (all momenta are incoming into the 1 PI vertices)

$$
\begin{aligned}
\left\langle\tilde{\psi}^{b}\left(p^{\prime}\right) \tilde{\psi}^{a}(p) \tilde{A}_{\mu}^{\alpha}(l)\right\rangle_{1 \mathrm{PI}} & \left.\equiv \frac{\delta}{\delta \tilde{A}_{\mu}^{\alpha}(l)} \frac{\delta}{\delta \tilde{\psi}^{a}(p)} \frac{\delta}{\delta \tilde{\psi}^{b}\left(p^{\prime}\right)} \Gamma[\phi, \psi, A, \ldots]\right|_{0} \\
& =(2 \pi)^{4} \delta^{(4)}\left(p^{\prime}+p+l\right) \widetilde{\Gamma}_{b a \alpha}^{\mu}\left(p^{\prime}, p, l\right) .
\end{aligned}
$$

The functional derivatives (which act always from the left) in (2.14) are taken at the "point" at which all fields vanish. Notice also the order of the fermionic variables and the "wrong" height of indices inside the bracket $\langle\cdot\rangle_{1 \mathrm{PI}}$. For the 1PI functions we will also use the notation

$$
\left\langle\tilde{\psi}^{b}\left(p^{\prime}\right) \tilde{\psi}^{a}(p) \tilde{A}_{\mu}^{\alpha}(l)\right\rangle_{1 \mathrm{PI}}=(2 \pi)^{4} \delta^{(4)}\left(p^{\prime}+p+l\right)\left\langle\tilde{\psi}^{b}\left(p^{\prime}\right) \tilde{\psi}^{a}(p) \tilde{A}_{\mu}^{\alpha}(l)\right\rangle_{\widetilde{1 \mathrm{PI}}} .
$$

Green's functions like (2.14) become "physical" when the background $\varphi$ is chosen so that the following condition is satisfied

$$
\left.\frac{\delta \Gamma[\phi, \psi, A, \ldots]}{\delta \phi^{i}(z)}\right|_{0}=0 .
$$

As we have already said, in studying renormalization we do not impose the above relation, treating $\varphi^{i}$ as arbitrary external parameters. Contributions of order $\hbar^{n}$ to the 1PI function are denoted $\widetilde{\Gamma}(\cdots)^{(n)}$, e.g.:

$$
\widetilde{\Gamma}_{b a \alpha}^{\mu}\left(p^{\prime}, p, l\right)=\sum_{n=0}^{\infty} \hbar^{n} \widetilde{\Gamma}_{b a \alpha}^{\mu}\left(p^{\prime}, p, l\right)^{(n)} .
$$


In what follows it will be convenient to further split $\widetilde{\Gamma}_{b a \alpha}^{\mu}\left(p^{\prime}, p, l\right)^{(1)}$ into the contribution of the counterterm diagrams and the sum of genuine one-loop diagram contributions. The latter will be denoted $\widetilde{\Gamma}_{b a \alpha}^{\mu}\left(p^{\prime}, p, l\right)^{(1 B)}$. If a given function is convergent by power-counting, the superscripts ${ }^{(1 B)}$ and ${ }^{(1)}$ are used interchangeably.

\section{The UV regularization}

As the UV regularization in our study of the renormalization of a general YM theory we choose (out of many other possibilities) the prescription which consists of modifying every derivative in the Lagrangian according to the rule

$$
\partial_{\mu} \rightarrow \exp \left\{\frac{\partial^{2}}{2 \Lambda^{2}}\right\} \partial_{\mu}
$$

The replacement (3.1) is to be done at the level of the Lagrangian densities (2.1) and (2.10); in the latter the BRST operations $s(\cdot)$ have to be carried out first (this should be considered a part of the regularization definition).

In the momentum space the above prescription is equivalent to the replacement

$$
k_{\mu} \rightarrow \mathcal{R}_{\mu}(k) \equiv \exp \left\{-\frac{k^{2}}{2 \Lambda^{2}}\right\} k_{\mu}
$$

Strictly speaking, the rule (3.2) should be applied to the Euclidean counterpart of the action (2.9), in the form $k_{E \mu} \rightarrow \exp \left\{+k_{E}^{2} /\left(2 \Lambda^{2}\right)\right\} k_{E \mu}$. Indeed, if (3.2) is applied literally to, say, the massless one-loop one-point function in the Minkowski space-time, the integral w.r.t. the time-like component of the momentum is badly divergent. By contrast, the corresponding Euclidean integral is undoubtedly convergent owing to the exponential damping factor (see below for consideration of an arbitrary diagram), which effectively restricts the integration region to Euclidean momenta obeying $k_{E} \lesssim \Lambda$; therefore we will call $\Lambda$ in the following the UV cutoff. The resulting amplitudes computed perturbatively in the Euclidean space are easily continued to the Minkowski space-time. (Such a treatement of the regularization does not preclude investigating non-perturbative effects, e.g. bound states, by summing infinite series of subtracted and continued to the Minkowski space Feynman diagrams.) In actual calculations we prefer to work with the Minkowski space-time Feynman rules. Therefore, instead of explicitly reformulating the theory in the Euclidean space, we work with the action (2.9) and the prescription (3.1), but perform in Feynman diagrams a formal Wick rotation, that is neglect contributions arising from (divergent) integrals over contours at infinity (in other words, all integrals over time-like components of loop momenta are in practice taken over the imaginary axis). In the perturbative expansion this procedure just implements the analytic continuation of the corresponding (convergent) integrals of the Euclidean version of theory. We also stress that in principle one could try to find a similar regularization acting directly in the Minkowski space-time by replacing the exponential in (3.1) with a polynomial, what gives a variant of the higher derivative regularization, see e.g. [53-55] - however, we prefer to work with the exponential form for the sake of calculational simplicity. 
In the more fundamental perspective (see section 9) we would like to treat the Euclidean version of the Lagrangian density modified according to the prescription (3.1) as a part of the complete Lagrangian density of an effective field theory for some fundamental finite theory of all interactions. The scale $\Lambda$ should be therefore identified with an intrinsic physical scale of the putative fundamental theory rather than with the scale introduced by the Wilsonian procedure of integrating out some high energy degrees of freedom, and the limit $\Lambda \rightarrow \infty$ should not be taken. Consistency of such an interpretation requires probably the fundamental theory to be formulated in the Euclidean space. The question then arises whether the prescription (3.1) in the effective theory can have a meaning also outside the perturbative expansion. Since the action has then a nonlocal character, standard arguments (appealing to the Osterwalder-Schrader theorem, whose status in YM theories remains, however, unclear) in favor of uniqueness of the analytical continuation to the Minkowski space-time of non-perturbatively determined Green's functions may not apply. Moreover with the exponential factors (3.3) not expanded, the propagators can, after continuation, develop unphysical poles, signaling potential problems. However, as will be seen (see the end of the next section), if the limit $\Lambda \rightarrow \infty$ is not taken, the (Euclidean) action (2.1) and (2.10) with the substitution (3.1) cannot be considered a complete action of the effective theory: further terms suppressed by inverse powers of $\Lambda$ must be added to it to restore the BRST symmetry for finite values of $\Lambda$. In the spirit of our further considerations we can therefore speculate that the complete Euclidean effective theory action is not sick when treated non-perturbatively and does allow for a unique continuation to the Minkowski space of the non-perturbative amplitudes.

The important virtue of the proposed prescription (3.1) is that it preserves the formal invariance of the path integral with respect to shifting fields by constant backgrounds, leading to the 1PI effective action $\Gamma$ satisfying the "translational Ward identity" [16]

$$
\Gamma[A, \psi, \phi, \ldots, \varphi]=\Gamma[A, \psi, \phi+\varphi, \ldots, 0]
$$

It is therefore applicable without modifications also to theories with spontaneous symmetry breaking by nonzero VEVs of scalar fields. On the practical side, the prescription (3.1) allows for an easy extraction of finite and divergent parts of amplitudes which can be automatized using standard computer packages for symbolic manipulations.

With the prescription (3.1) the propagators of vector bosons (in the Landau gauge), fermions, scalars and ghosts take respectively the forms:

$$
\begin{aligned}
i D_{\mu_{1} \mu_{2}}^{\alpha_{1} \alpha_{2}}(k ; \Lambda) & =\left[\frac{-i}{\mathcal{R}^{2}(k)-m_{V}^{2}}\right]^{\alpha_{1} \alpha_{2}}\left(\eta_{\mu_{1} \mu_{2}}-\frac{k_{\mu_{1}} k_{\mu_{2}}}{k^{2}}\right), \\
i S^{a_{1} a_{2}}(p ; \Lambda) & =\left[\frac{i}{\mathcal{R}(p)-\widehat{m}_{F}} C^{-1}\right]^{a_{1} a_{2}}, \\
i \Delta^{i_{1} i_{2}}(q ; \Lambda) & =\left[\frac{i}{\mathcal{R}^{2}(q)-m_{S}^{2}}\right]^{i_{1} i_{2}}, \\
i D_{g h \beta}^{\alpha}(k ; \Lambda) & =\frac{i}{\mathcal{R}^{2}(k)} \delta_{\beta}^{\alpha} .
\end{aligned}
$$


The mixed scalar-vector propagator vanishes owing to the choice of the Landau gauge (2.11). We also list the vertices which get modified by the prescription (3.1):

$$
\begin{aligned}
& \tilde{\mathcal{L}}_{A A A}(\{k\})= \frac{i}{3 !} e_{\alpha_{1} \alpha_{2} \alpha_{3}}\left\{\left[\mathcal{R}\left(k_{3}\right)-\mathcal{R}\left(k_{2}\right)\right]^{\mu_{1}} \eta^{\mu_{3} \mu_{2}}+\left[\mathcal{R}\left(k_{1}\right)-\mathcal{R}\left(k_{3}\right)\right]^{\mu_{2}} \eta^{\mu_{1} \mu_{3}}\right. \\
&\left.+\left[\mathcal{R}\left(k_{2}\right)-\mathcal{R}\left(k_{1}\right)\right]^{\mu_{3}} \eta^{\mu_{2} \mu_{1}}\right\} \tilde{A}_{\mu_{1}}^{\alpha_{1}}\left(k_{1}\right) \tilde{A}_{\mu_{2}}^{\alpha_{2}}\left(k_{2}\right) \tilde{A}_{\mu_{3}}^{\alpha_{3}}\left(k_{3}\right), \\
& \tilde{\mathcal{L}}_{A \phi \phi}(\{k\})= \frac{i}{2 !}\left[\mathcal{R}\left(k_{2}\right)-\mathcal{R}\left(k_{1}\right)\right]^{\mu}\left(\mathcal{T}_{\alpha}\right)_{i_{1} i_{2}} \tilde{A}_{\mu}^{\alpha}\left(k_{3}\right) \tilde{\phi}^{i_{1}}\left(k_{1}\right) \tilde{\phi}^{i_{2}}\left(k_{2}\right), \\
& \tilde{\mathcal{L}}_{A \bar{\omega} \omega}(\{k\})=-i \mathcal{R}^{\mu}\left(k_{1}\right) e_{\beta \alpha_{2}}^{\alpha_{1}} \tilde{A}_{\mu}^{\beta}\left(k_{3}\right) \tilde{\bar{\omega}}_{\alpha_{1}}\left(k_{1}\right) \tilde{\omega}^{\alpha_{2}}\left(k_{2}\right) .
\end{aligned}
$$

We have used here the notation

$$
I_{0}^{\Lambda}=\sum_{\left\{\Phi_{1}, \ldots, \Phi_{n}\right\}} \int \prod_{i=1}^{n} \mathrm{~d}^{4} k_{i} \tilde{\mathcal{L}}_{\Phi_{1} \cdots \Phi_{n}}\left(k_{1}, \ldots, k_{n}\right)(2 \pi)^{4} \delta^{(4)}\left(k_{1}+\cdots+k_{n}\right) .
$$

The remaining vertices having $n \geq 3$ are not modified. ${ }^{3}$

To see that indeed all relevant diagrams are regularized by the prescription (3.1), consider a 1PI diagram $\gamma$ consisting of $V_{i}$ vertices of type $i$ involving (prior to regularization) $d_{i}$ derivatives and to which $n_{i \Phi}$ lines of fields of type $\Phi$ are attached, $I_{\Phi}$ internal and $E_{\Phi}$ external lines of type $\Phi$. The corresponding integrand (after formal Wick rotation) acquires the factor $\exp \left(\bar{\omega}(\gamma) k_{E}^{2} / 2 \Lambda^{2}\right)$, where

$$
\bar{\omega}(\gamma) \equiv \sum_{\Phi} I_{\Phi}\left(2 s_{\Phi}-2\right)+\sum_{i} V_{i} d_{i}
$$

(the factor $s_{\Phi}$ characterizes the $\Phi$ line propagator which behaves as $k_{E}^{2 s_{\Phi}-2}$ as $k_{E} \rightarrow \infty$ ). Obviously, a diagram $\gamma$ gets regularized if $\bar{\omega}(\gamma)<0$. Moreover, since $\omega(\gamma)=4 L+\bar{\omega}(\gamma)$, where $L \geq 0$ is the number of loops and $\omega(\gamma)$ is the textbook degree of superficial divergence, ${ }^{4}$ it follows that superficially convergent diagrams (of $\omega(\gamma)<0$ ) necessarily have $\bar{\omega}(\gamma)<0$. Using the standard identities one gets that

$$
\bar{\omega}(\gamma)=-4(L-1)-\sum_{\Phi} E_{\Phi}\left(1+s_{\Phi}\right)-\sum_{i} V_{i} \Delta_{i}
$$

where $\Delta_{i}=4-d_{i}-\sum_{\Phi} n_{i \Phi}\left(1+s_{\Phi}\right)$. This shows that in renormalizable theories, in which all vertices have $\Delta_{i} \geq 0$, unregulated by the prescription (3.1) remain only oneloop $(L=1)$ vacuum $\left(E_{\Phi}=0\right)$ diagrams which cannot appear in physically interesting amplitudes as divergent subdiagrams. All other diagrams arising in renormalizable theories get regularized.

Computation of diagrams regularized with the help of the prescription (3.2) is based on the following expansion

$$
\frac{i}{\mathcal{R}^{2}(k)-m^{2}}=e^{k^{2} / \Lambda^{2}} \frac{i}{k^{2}-m^{2}} \sum_{n=0}^{\infty}\left[\frac{m^{2}}{m^{2}-k^{2}}\left(1-e^{k^{2} / \Lambda^{2}}\right)\right]^{n},
$$

\footnotetext{
${ }^{3}$ The two-point vertex $\tilde{\mathcal{L}}_{K_{\alpha}^{\mu} \omega^{\beta}}$ is omitted here as it does not contribute to loop 1PI diagrams. For the same reason propagators involving the Nakanishi-Lautrup multipliers $h_{\alpha}$ are omitted.

${ }^{4}$ See e.g. ref. [17].
} 
( $k$ may stand for a sum of several loop and external line momenta). It is clear that in the Euclidean space, for $k^{2} \rightarrow-k_{E}^{2}$, the expansion (3.9) would be absolutely convergent. In particular, owing to the growing inverse powers of $m^{2}-k^{2}$ in successive terms, for a given one-loop diagram only a finite number of terms yield integrals that are divergent when the factors $e^{k^{2} / \Lambda^{2}}\left(1-e^{k^{2} / \Lambda^{2}}\right)^{n}$ are omitted. The remaining terms are integrable without these factors which implies that their contributions vanish in the limit $\Lambda \rightarrow \infty$. Thus the practical recipe for computing diagrams regularized with the help of (3.2) consists of the following steps (see also B): i) expanding all regularized propagators as in (3.9), ii) combining denominators using the standard trick introducing integrals over Feynman parameters $\alpha_{i}$, iii) shifting and formally Wick-rotating the momenta, iv) expanding the exponential factors in powers of external momenta, $v$ ) performing integrals over angular variables. After these steps every one-loop diagram gets represented in the form of the confluent hypergeometric function

$$
U(a, b, z)=\frac{1}{\Gamma(a)} \int_{0}^{\infty} \mathrm{d} t t^{a-1}(1+t)^{b-a-1} \exp (-z t),
$$

in which $a$ and $b$ are some real numbers, $t \propto k_{E}^{2}$ and $z$ is the ratio of a linear combination of masses squared and external momenta squared weighed by the Feynman parameters $\alpha_{i}$ and of $\Lambda^{2}$. One is therefore led to study the limit of $z \rightarrow 0$ of $U(a, b, z)$ which can be extracted using the well known formulae [18]. In this way one-loop diagrams get represented in the standard form of integrals over Feynman parameters.

Although this is not necessary for one-loop calculations, we note that in general extraction of the $\Lambda \rightarrow \infty$ asymptotics can be efficiently done by exploiting a theorem by Handelsman and Lew [19] which relates the requisite coefficients in the asymptotics of the Laplace transform of the general form

$$
L[f, z]=\int_{0}^{\infty} \mathrm{d} t f(t) e^{-z t}
$$

directly to the coefficients of the $t \rightarrow \infty$ asymptotics of the function $f(t)$ and to constant terms in the Laurent expansions of (the analytic continuation of) the Mellin transform

$$
M[f, z]=\int_{0}^{\infty} \mathrm{d} t f(t) t^{z-1} .
$$

around its poles. Thus, the Handelsman-Lew theorem is crucial for finding the asymptotic form of multi-loop diagrams, which cannot be expressed in terms of the function (3.10).

\section{The subtraction procedure}

The UV cutoff introduced in section 3 explicitly breaks the BRST symmetry $-s\left(I_{0}^{\Lambda}\right) \neq 0$, where $I_{0}^{\Lambda}$ is the action (2.9) modified according to the prescription (3.1). Consistency of the quantized gauge theory does not require, however, BRST invariance of $I_{0}^{\Lambda}$, but only BRST invariance of the 1PI effective action $\Gamma$ — the functional generating one-particle irreducible (1PI) Green's functions. This can be restored by using the general methodology based on the Quantum Action Principle [6-9] (see also [20, 21] for reviews). In practical terms it 
consists of starting with the local BRST invariant action expressed in terms of renormalized fields and parameters and in making in the computed Green's functions (or the effective action) order by order in the loop expansion appropriate subtractions in such a way, that the Zinn-Justin (ZJ) identity [22]

$$
\mathcal{S}(\Gamma)=0,
$$

in which $\mathcal{S}(\cdot)$ is the differential operator whose action on an arbitrary functional $F$ of fields and antifields is given by ${ }^{5}$

$$
\mathcal{S}(F) \equiv \frac{\delta F}{\delta K_{\alpha}^{\mu}} \cdot \frac{\delta F}{\delta A_{\mu}^{\alpha}}+\frac{\delta F}{\delta K_{i}} \cdot \frac{\delta F}{\delta \phi^{i}}+\frac{\delta F}{\delta \bar{K}_{a}} \cdot \frac{\delta F}{\delta \psi^{a}}+\frac{\delta F}{\delta L_{\alpha}} \cdot \frac{\delta F}{\delta \omega^{\alpha}}+h_{\alpha} \cdot \frac{\delta F}{\delta \bar{\omega}_{\alpha}} .
$$

is satisfied (up to higher order terms) by the subtracted effective action $\Gamma$. Within the general framework the possibility to restore BRST invariance of the effective action (in nonanomalous theories) in this way was first demonstrated in [15] using the BPHZ scheme [23] in which subtractions are made directly in integrands of the integrals corresponding to Feynman diagrams and thus no explicit regulator is introduced. This approach is usually used in formal proofs of existence (within the perturbation theory) of unitary gauge theories for which no symmetry preserving regularization is available [24-27]; some practical calculations within the Standard Model (SM) based on this approach can be found in [28-31].

The general QAP methodology can obviously be applied also in conjunction with any explicit BRST symmetry violating regulator. In such an approach one constructs order by order in the perturbative expansion the counterterms: the divergent (as the regulator is removed) ones, which in our scheme will be uniquely determined by the regularization and the adopted "minimal" subtraction prescription, and the additional finite counterterms restoring the ZJ identity. This approach has been used in particular to renormalize YM theories with chiral fermions using DimReg and the original 't Hooft-Veltman definition of the $\gamma^{5}$ matrix which avoids inconsistencies [32-34] but breaks the BRST symmetry already at one-loop. The full set of one-loop counterterms was determined in specific models [35], including supersymmetric ones [36] as well as in an arbitrary renormalizable gauge theory without scalars [10].

In this paper we apply this approach to the regularization of a general renormalizable YM theory by the explicit UV momentum cutoff defined in section 3 (see [37-43] for partially related applications in the context of the Wilson-Polchinski renormalization group). Below we recall the general procedure based on the QAP and specify our way of fixing its arbitrariness (our renormalization conditions).

As said, the starting point is the regularized action $I_{0}^{\Lambda}$ obtained by applying the prescription (3.1) to the local BRST symmetric action $I_{0}$ defined by (2.9). All fields and parameters of $I_{0}$ have the interpretation of renormalized quantities. The action $I_{0}$ is such that $\mathcal{S}\left(I_{0}\right)=0$ and satisfies a number of additional conditions listed in appendix A.1. Since

$$
\Gamma\left[I_{0}^{\Lambda}\right] \equiv \Gamma_{0}^{\Lambda}=I_{0}^{\Lambda}+\mathcal{O}(\hbar),
$$

the "asymptotic part" of $\Gamma_{0}^{\Lambda}$ (denoted $\Gamma_{0}$ ) obtained by neglecting all terms which vanish in the limit $\Lambda \rightarrow \infty$ satisfies the ZJ identity (4.1) up to terms of order $\hbar$.

\footnotetext{
${ }^{5}$ We use the notation $k \cdot g \equiv \int \mathrm{d}^{4} x k(x) g(x)$.
} 
We now show that having a local action $I_{n}$ (with all counterterms up to the order $\hbar^{n}$ included) satisfying the conditions of appendix A.1 and such that in the asymptotic part $\Gamma_{n}$ of $\Gamma_{n}^{\Lambda} \equiv \Gamma\left[I_{n}^{\Lambda}\right]$

$$
\Gamma_{n}=I_{0}+\sum_{k=1}^{\infty} \hbar^{k} \Gamma_{n}^{(k)}
$$

the functionals $\Gamma_{n}^{(k)}$ are already $\Lambda$-independent for $k \leq n$ and

$$
\mathcal{S}\left(\Gamma_{n}\right)=\hbar^{n+1} \Omega_{n}+\mathcal{O}\left(\hbar^{n+2}\right),
$$

it is possible to construct $I_{n+1}$ extending these results to the next order in $n$. Useful in this, in addition to the operator (4.2), is also its linearized version $\mathcal{S}_{F}$ [21] defined by $\mathcal{S}(F+\varepsilon G)=\mathcal{S}(F)+\varepsilon \mathcal{S}_{F}(G)+\mathcal{O}\left(\varepsilon^{2}\right)$, whose explicit form reads

$$
\begin{aligned}
\mathcal{S}_{F}= & \frac{\delta F}{\delta K_{\alpha}^{\mu}} \cdot \frac{\delta}{\delta A_{\mu}^{\alpha}}+\frac{\delta F}{\delta K_{i}} \cdot \frac{\delta}{\delta \phi^{i}}+\frac{\delta F}{\delta \bar{K}_{a}} \cdot \frac{\delta}{\delta \psi^{a}}+\frac{\delta F}{\delta L_{\alpha}} \cdot \frac{\delta}{\delta \omega^{\alpha}}+h_{\alpha} \cdot \frac{\delta}{\delta \bar{\omega}_{\alpha}} \\
& +\frac{\delta F}{\delta A_{\mu}^{\alpha}} \cdot \frac{\delta}{\delta K_{\alpha}^{\mu}}+\frac{\delta F}{\delta \phi^{i}} \cdot \frac{\delta}{\delta K_{i}}+\frac{\delta F}{\delta \psi^{a}} \cdot \frac{\delta}{\delta \bar{K}_{a}}+\frac{\delta F}{\delta \omega^{\alpha}} \cdot \frac{\delta}{\delta L_{\alpha}} .
\end{aligned}
$$

The operations $\mathcal{S}(\cdot)$ and $\mathcal{S}_{F}$ have two important properties [21]. Firstly,

$$
\mathcal{S}_{F} \mathcal{S}(F)=0 .
$$

for any functional $F$. Secondly, if $\mathcal{S}(F)=0$, then

$$
\mathcal{S}_{F}^{2}=0 \text {. }
$$

In particular, $\mathcal{S}_{I_{0}}^{2}=0$.

It is the well known property of the ordinary renormalization procedure that the lowest order divergent (in the infinite cutoff limit) part of $\Gamma_{n}$, that is $\Gamma_{n}^{(n+1) \text { div }}$, is an integral of a local operator which can be removed by adding to $I_{n}$ appropriate counterterms. Similarly, the QAP guarantees [6-9], that $\Omega_{n}$ in (4.4) is an integral of a local operator (of ghost number 1 and dimension $\leq 5)$. Moreover, using the identity (4.6) applied to $F=\Gamma_{n}$ in conjunction with the expansion $\mathcal{S}_{\Gamma_{n}}=\mathcal{S}_{I_{0}}+\mathcal{O}(\hbar)$ one learns that $\Omega_{n}$ satisfies the WessZumino consistency condition (WZCC)

$$
\mathcal{S}_{I_{0}} \Omega_{n}=0 .
$$

Restoring the BRST invariance of $\Gamma$ in the order $\hbar^{n+1}$ relies on the possibility of representing $\Omega_{n}$ in a cohomologically trivial form

$$
\Omega_{n}=\mathcal{S}_{I_{0}} \mathscr{C}_{n}
$$

with $\mathscr{C}_{n}$ being the integral of some local operator (of ghost number 0 and dimension $\leq 4$ ), which can therefore be used as an additional (symmetry restoring) counterterm. This is so if the representation of the gauge group realized on fermionic fields fulfills (cf. eq. (2.5))

$$
\operatorname{tr}\left(\mathfrak{f}_{\alpha}\left\{\mathfrak{f}_{\beta}, \mathfrak{f}_{\gamma}\right\}\right)=0
$$


for all triplets $(\alpha, \beta, \gamma)$ of the gauge indices. ${ }^{6}$

In the "algebraic renormalization" framework usually explicit renormalization conditions are used to fix the counterterm $\mathscr{C}_{n}$ [21]. Here, aiming at constructing a massindependent renormalization scheme, we adopt a two-step procedure instead. In the first step a local action

$$
\tilde{I}_{n}=I_{n}-\hbar^{n+1} \Gamma_{n}^{(n+1) \operatorname{div}},
$$

is constructed with the divergent part $\Gamma_{n}^{(n+1) \text { div }}$ defined in the spirit of (the modified) minimal subtraction as the "pure divergence", i.e. by imposing the condition

$$
\left.\left.\Gamma_{n}^{(n+1) \operatorname{div}}\right|_{\delta_{\Lambda}=0}\right|_{\Lambda^{2}=0}=0
$$

in which $\delta_{\Lambda}$ is the "basic logarithmic divergence"

$$
\delta_{\Lambda} \equiv \ln \frac{\Lambda^{2}}{\mu^{2}}-1-\gamma_{\mathrm{E}}-\ln 2=\ln \frac{\bar{\Lambda}^{2}}{\mu^{2}} .
$$

The arbitrary scale $\mu$ is introduced on dimensional ground to render the subtraction procedure mass-independent. The "asymptotic" (in the sense explained above) part $\tilde{\Gamma}_{n}$ of the effective action $\tilde{\Gamma}_{n}^{\Lambda} \equiv \Gamma\left[\tilde{I}_{n}^{\Lambda}\right]$ obtained from the regularized version $\tilde{I}_{n}^{\Lambda}$ of $\tilde{I}_{n}$ has then the form ${ }^{7}$

$$
\tilde{\Gamma}_{n}=\Gamma_{n}-\hbar^{n+1} \Gamma_{n}^{(n+1) \operatorname{div}}+\mathcal{O}\left(\hbar^{n+2}\right),
$$

and it is easy to see that

$$
\mathcal{S}\left(\tilde{\Gamma}_{n}\right)=\hbar^{n+1} \tilde{\Omega}_{n}+\mathcal{O}\left(\hbar^{n+2}\right),
$$

where $\tilde{\Omega}_{n}$ is related to $\Omega_{n}$ in (4.4) by

$$
\tilde{\Omega}_{n} \equiv \Omega_{n}-\mathcal{S}_{I_{0}} \Gamma_{n}^{(n+1) \mathrm{div}} .
$$

As all $\Lambda$-dependent terms in $\tilde{\Gamma}_{n}$ are at least of order $\hbar^{n+2}$, eq. (4.15) means that $\tilde{\Omega}_{n}$ is $\Lambda$-independent. Furthermore, (4.9) (if true) implies that

$$
\tilde{\Omega}_{n}=-\mathcal{S}_{I_{0}} \delta_{b} \Gamma_{n}^{(n+1)} .
$$

with $\delta_{b} \Gamma_{n}^{(n+1)}$ being the integral of a (cutoff-independent) local operator (of ghost number 0 and dimension $\leq 4)$. Regularized version $I_{n+1}^{\Lambda}$ of the next order local action

$$
I_{n+1}=\tilde{I}_{n}+\hbar^{n+1} \delta_{b} \Gamma_{n}^{(n+1)}=I_{n}+\hbar^{n+1}\left\{\delta_{b} \Gamma_{n}^{(n+1)}-\Gamma_{n}^{(n+1) \operatorname{div}}\right\}
$$

\footnotetext{
${ }^{6}$ For semisimple gauge groups the only cohomologically non-trivial solution to the WZCC (4.8) is the Adler-Bell-Jackiw anomaly, which vanishes to all orders if (4.10) holds (see e.g. [21]). Additional (Abelian) anomalies that could potentially appear in the case of non-semisimple gauge groups [44] are excluded if the Abelian antighost equation (A.6) is imposed as one of the conditions defining the theory (see [45] and references therein).

${ }^{7}$ The form (4.14) is correct, because quadratic divergences are independent of external momenta. For this reason, terms of the form

$$
\Lambda^{2} \exp \left\{-\frac{\ell^{2}}{\Lambda^{2}}\right\}=\Lambda^{2}-\ell^{2}+\mathcal{O}\left(\Lambda^{-2}\right)
$$

will not be produced by the prescription (3.1).
} 
leads then to $\Gamma_{n+1}^{\Lambda} \equiv \Gamma\left[I_{n+1}^{\Lambda}\right]$ whose asymptotic part $\Gamma_{n+1}$ reads

$$
\Gamma_{n+1}=\Gamma_{n}+\hbar^{n+1}\left\{\delta_{b} \Gamma_{n}^{(n+1)}-\Gamma_{n}^{(n+1) \operatorname{div}}\right\}+\mathcal{O}\left(\hbar^{n+2}\right),
$$

and breaks the ZJ identity (4.1) only at the $\hbar^{n+2}$ order:

$$
\mathcal{S}\left(\Gamma_{n+1}\right)=\hbar^{n+1}\left\{\Omega_{n}-\mathcal{S}_{I_{0}}\left[\Gamma_{n}^{(n+1) \operatorname{div}}-\delta_{b} \Gamma_{n}^{(n+1)}\right]\right\}+\mathcal{O}\left(\hbar^{n+2}\right)=0+\mathcal{O}\left(\hbar^{n+2}\right) .
$$

To complete the inductive step it is still necessary to show that $I_{n+1}$ satisfies also all the auxiliary conditions (A.1)-(A.8). This is done in appendix A.2.

Due to the non-triviality of $\operatorname{ker} \mathcal{S}_{I_{0}}$, the counterterm $\delta_{b} \Gamma_{n}^{(n+1)}$ is not uniquely determined by the condition (4.17) - any functional $v_{0}$ belonging to $\mathcal{V} \cap \operatorname{ker} \mathcal{S}_{I_{0}}$ can be added to it. Here $\mathcal{V}$ denotes the vector space of integrals of local operators of dimension $\leq 4$ and zero ghost number satisfying the homogeneous versions of the conditions (A.1)-(A.8) and having other symmetries of $I_{0}$. It is easy to check that any $v_{0} \in \mathcal{V} \cap \operatorname{ker} \mathcal{S}_{I_{0}}$ has the form

$$
\begin{aligned}
v_{0}=\int \mathrm{d}^{4} x\left\{\mathbb{E}_{\beta_{S}}^{\kappa_{S}}\left[\left(K_{\kappa_{S}}^{\mu}-\partial^{\mu} \bar{\omega}_{\kappa_{S}}\right) \partial_{\mu} \omega^{\beta_{S}}+\frac{\delta I_{0}^{G I}}{\delta A_{\mu}^{\kappa_{S}}} A_{\mu}^{\beta_{S}}\right]+\right. \\
\left.-\frac{1}{2} z_{\alpha \beta}^{A} F_{\mu \nu}^{\alpha} F^{\beta \mu \nu}+z_{i j}^{\phi}\left(D_{\mu} \phi\right)^{i}\left(D^{\mu} \phi\right)^{j}+i\left(\bar{\psi} \gamma^{\mu}\right)^{a} z_{a b}^{\psi}\left(D_{\mu} \psi\right)^{b}-w(\phi, \psi)\right\}
\end{aligned}
$$

with the matrices $\mathbb{E}, z$ (of course, $z^{\psi}=z^{F} P_{L}+z^{F *} P_{R}$ ) and the polynomial $w(\phi, \psi)$ constrained by the global symmetries of $I_{0}$ (including those which belong to the gauge group); moreover $z_{i j}^{\phi}=0$ if either $i$ or $j$ corresponds to the Stueckelberg scalar and $w(\phi, \psi)$ is independent of the Stueckelberg fields.

Remembering that the tree-level action $I_{0}$ is (up to a rescaling of field) the most general functional consistent with the power-counting and a given set of symmetries, it is easy to check that eq. (4.21) can be can be rewritten in the form (here $g^{C}$ denotes collectively all parameters of $I_{0}$ except for components of the background $\varphi$, i.e. couplings constants and explicit mass parameters)

$$
v_{0}=-\left\{\mathfrak{B}^{C} \frac{\partial}{\partial g^{C}}-\mathcal{N}_{\phi}\left(z^{\phi}\right)-\mathcal{N}_{\psi}\left(z^{\psi}\right)-\mathcal{N}_{\omega}\left(z^{A}\right)-\mathcal{N}_{A}\left(\overline{\mathbb{E}}+z^{A}\right)\right\} I_{0}
$$

where

$$
\begin{aligned}
& \mathcal{N}_{\phi}\left(z^{\phi}\right)=\left(z^{\phi}\right)^{i}{ }_{j}\left\{(\phi+\varphi)^{j} \cdot \frac{\delta}{\delta \phi^{i}}-K_{i} \cdot \frac{\delta}{\delta K_{j}}\right\}, \\
& \mathcal{N}_{\psi}\left(z^{\psi}\right)=\left(z^{\psi}\right)^{a}{ }_{b}\left\{\psi^{b} \cdot \frac{\delta}{\delta \psi^{a}}-\bar{K}_{a} \cdot \frac{\delta}{\delta \bar{K}_{b}}\right\}, \\
& \mathcal{N}_{\omega}(z)=z^{\alpha}{ }_{\beta}\left\{\omega^{\beta} \cdot \frac{\delta}{\delta \omega^{\alpha}}-L_{\alpha} \cdot \frac{\delta}{\delta L_{\beta}}\right\}, \\
& \mathcal{N}_{A}(z)=z^{\alpha}{ }_{\beta}\left\{A_{\mu}^{\beta} \cdot \frac{\delta}{\delta A_{\mu}^{\alpha}}-K_{\alpha}^{\mu} \cdot \frac{\delta}{\delta K_{\beta}^{\mu}}-\bar{\omega}_{\alpha} \cdot \frac{\delta}{\delta \bar{\omega}_{\beta}}-h_{\alpha} \cdot \frac{\delta}{\delta h_{\beta}}\right\}
\end{aligned}
$$

are the "counting operators" [21], $\overline{\mathbb{E}}$ equals $\mathbb{E}$ for non-Abelian indices and vanishes otherwise

$$
\overline{\mathbb{E}}_{\beta}^{\alpha}=\delta_{\kappa_{S}}^{\alpha} \mathbb{E}_{\theta_{S}}^{\kappa_{S}} \delta_{\beta}^{\theta_{S}},
$$


while the coefficients $\mathfrak{B}^{C}$ satisfy the relations

$$
\mathfrak{B}^{C} \frac{\partial}{\partial g^{C}} T_{\alpha}=\left[z^{A}\right]_{\alpha}^{\gamma} T_{\gamma}, \quad \text { for } \quad T_{\gamma}=\mathcal{T}_{\gamma}, t_{\gamma}, e_{\gamma},
$$

and

$$
\mathfrak{B}^{C} \frac{\partial}{\partial g} \bar{P}_{\alpha_{A}}=\left[z^{A}\right]_{\alpha_{A}}^{\delta_{A}} \bar{P}_{\delta_{A}}
$$

Of course, the coefficients $\mathfrak{B}^{C}$ corresponding to non-gauge couplings $g^{C}$ (which parametrize the $w(\phi, \psi)$ polynomial) are not constrained by the relations (4.25)-(4.26).

The form (4.22) of $v_{0}$ implies that this functional can be obtained from $I_{0}$ by an infinitesimal "finite renormalization" of its fields and couplings $g^{C}$. This shows that the necessity of fixing the freedom in the form of the counterterms $\delta_{b} \Gamma_{n}^{(n+1)}$ is equivalent to the usual necessity of specifying the renormalization conditions.

In our approach we impose the implicit renormalization conditions by requiring that the counterterms $\delta_{b} \Gamma_{n}^{(n+1)}$ belong to a subspace $\mathcal{W} \subset \mathcal{V}$ which is complementary to the subspace $\mathcal{V} \cap \operatorname{ker} \mathcal{S}_{I_{0}}$, that is such that $\mathcal{V}=\left[\mathcal{V} \cap \operatorname{ker} \mathcal{S}_{\mathcal{I}_{0}}\right] \oplus \mathcal{W}$. Different choices of $\mathcal{W}$ correspond to different mass-independent renormalization schemes. Since a generic element $v$ of $\mathcal{V}$ is of the form

$$
v=\mathcal{J}[A, \phi, \psi]+\tilde{\mathbb{E}}_{\beta_{S}}^{\kappa_{S}} \int \mathrm{d}^{4} x\left[K_{\kappa_{S}}^{\mu}-\partial^{\mu} \bar{\omega}_{\kappa_{S}}\right] \partial_{\mu} \omega^{\beta_{S}},
$$

where the functional $\mathcal{J}$ is independent of the Stueckelberg fields and constrained by power-counting and global (gauge and other) symmetries of $I_{0}$, it is easy to see that one (particularly natural) choice is the subspace $\mathcal{W}$ spanned by the following integrated operators (in the symbolic form)

$$
\begin{gathered}
\delta_{b} \Gamma_{n}^{(n+1)} \in \int\left(\partial^{\mu} A_{\mu}\right)\left(\partial^{\nu} A_{\nu}\right) \oplus A_{\mu} A^{\mu} \oplus A_{\mu} \bar{\psi} \gamma^{\mu} P_{L} \psi \oplus A_{\mu} \bar{\psi} \gamma^{\mu} P_{R} \psi \oplus \phi \phi A_{\mu} A^{\mu} \oplus \\
\oplus A_{\mu} \partial^{\mu} \phi \oplus \phi A_{\mu} \partial^{\mu} \phi \oplus \phi A_{\mu} A^{\mu} \oplus A A \partial A \oplus A A A A
\end{gathered}
$$

in which each component represents a set of operators with all possible assignments of the "color" (and "flavor") indices. In the last two terms suppressed Lorentz indices have to be contracted in a Lorentz-invariant way. The counterterm (4.27) vanishes for $A=0$ and does not involve the $A_{\mu} \partial_{\nu} \partial^{\nu} A^{\mu}$ operator nor the Stueckelberg fields. We will call this choice the $\Lambda$ - $\overline{\mathrm{MS}}$ scheme.

As a result of the procedure outlined above the action $I_{\infty}^{\Lambda}$ is constructed which, modulo exponents introduced according to the prescription (3.1), has a renormalizable form but is obviously not BRST symmetric. In typical applications of the procedure, mentioned at the beginning of this section, the structure of the resulting BRST symmetry violating counterterms is not very interesting in itself - the counterterms serve only as a technical mean to consistently calculate finite amplitudes satisfying the appropriate identities (which embody the requirements of the BRST invariance). Therefore one usually does not exploit the fact that, as will be shown in section 7 , the action $I_{\infty}^{\Lambda}$, can be given the interpretation of the "bare" action $I_{\mathrm{B}}$ expressed in terms of the "bare" parameters. This fact, however, will be crucial in discussing our view on the hierarchy problem in section 9 . 
Before closing this section an important comment must be made. From the above description of the procedure for constructing counterterms it is clear that the full BRST invariance of the effective action $\Gamma$ (i.e. the ZJ identity) is recovered only in the strict limit $\Lambda \rightarrow \infty$. This is perfectly fine if one does not ask about the origin of the low energy field theory model and is interested only in obtaining renormalized (finite) amplitudes satisfying the requirements of the BRST symmetry. On the other hand, if the bare action and the cutoff $\Lambda$ are to be given a physical meaning (and the limit $\Lambda \rightarrow \infty$ is not to be taken), one has to assume that the complete bare action $I_{\mathrm{B}}$ has additional terms, suppressed by inverse powers of $\Lambda$, which are not obtained with the help of the outlined procedure applied to the regularized renormalizable action (2.9), and which conspire to restore the full BRST invariance of the amplitudes. Indeed, the experimental limit on the photon mass $M_{\gamma}<10^{-18} \mathrm{eV}$ [46] does not leave room for BRST (gauge) symmetry breaking at order $M_{\mathrm{top}}^{4} / \Lambda^{2}$ (or $M_{W}^{4} / \Lambda^{2}$ ), even for $\Lambda$ as high as the Planck scale. As pointed out in the discussion of the regularization prescription (3.1), in the complete Euclidean action additional terms postulated here may be also important in the problem of the non-perturbative continuation to the Minkowski space-time. We do not attempt here to determine the form of these terms. We only point out that such a situation can be somewhat analogous to the one encountered in superstring theory: while the anomaly is shown to cancel out exactly at the string theory level, the minimal supergravity - the effective low energy theory of massless string excitations derived from string tree-level amplitudes is anomalous. Making it anomaly-free requires modifying the field strength $H=d B$ by adding a term which originates from one-loop string amplitudes; this correction taken alone breaks supersymmetry; restoring supersymmetry reintroduces, in turn, the anomaly and so on.

\section{Determination of the BRST symmetry restoring counterterms}

At the one-loop order the ZJ identity (4.1) is equivalent to the condition

$$
\mathcal{S}_{I_{0}} \Gamma^{(1)}=0
$$

where $\Gamma^{(1)} \equiv \Gamma_{1}^{(1)}$ is the one-loop contribution to the renormalized 1PI effective action (for the notation, see (4.3)). In our renormalization scheme (see section 4) the BRST symmetry restoring counterterm $\delta_{b} \Gamma_{0}^{(1)}$ must be of the form (4.27). In order to determine the coefficients of its individual terms it is sufficient to consider the derivative of (5.1) with respect to the ghost field restricted to the "physical submanifold"

$$
\omega^{\alpha}=\bar{\omega}_{\alpha}=K_{\alpha}^{\mu}=K_{i}=\bar{K}_{a}=L_{\alpha}=h_{\alpha}=0 .
$$

In the momentum space, cf. eq. (2.13), the resulting identity reads ${ }^{8}$

$$
\begin{aligned}
& i l_{\mu} \frac{\delta \Gamma_{p h}^{(1)}}{\delta \tilde{A}_{\mu}^{\gamma}(l)}+\int \mathrm{d}^{4} p e^{\alpha}{ }_{\gamma \beta} \tilde{A}_{\mu}^{\beta}(p-l) \frac{\delta \Gamma_{p h}^{(1)}}{\delta \tilde{A}_{\mu}^{\alpha}(p)}+\int \mathrm{d}^{4} p\left[t_{\gamma} \tilde{\psi}(p-l)\right]^{a} \frac{\delta \Gamma_{p h}^{(1)}}{\delta \tilde{\psi}^{a}(p)}+ \\
& +\int \mathrm{d}^{4} p\left[\mathcal{T}_{\gamma} \tilde{\phi}(p-l)\right]^{i} \frac{\delta \Gamma_{p h}^{(1)}}{\delta \tilde{\phi}^{i}(p)}+\left[\mathcal{T}_{\gamma} \varphi\right]^{i} \frac{\delta \Gamma_{p h}^{(1)}}{\delta \tilde{\phi}^{i}(l)}+
\end{aligned}
$$

${ }^{8}$ To simplify the notation we write $\Gamma_{p h}^{(1)}=\left.\Gamma^{(1)}\right|_{p h}$. 


$$
\begin{aligned}
& +\int \frac{\mathrm{d}^{4} p}{(2 \pi)^{4}} \frac{\delta I_{0}^{G I}}{\delta \tilde{A}_{\mu}^{\alpha}(p)}\left[\frac{\delta}{\delta \tilde{\omega}^{\gamma}(l)} \frac{\delta \Gamma^{(1)}}{\delta \tilde{K}_{\alpha}^{\mu}(-p)}\right]_{p h}-\int \frac{\mathrm{d}^{4} p}{(2 \pi)^{4}} \frac{\delta I_{0}^{G I}}{\delta \tilde{\psi}^{a}(p)}\left[\frac{\delta}{\delta \tilde{\omega}^{\gamma}(l)} \frac{\delta \Gamma^{(1)}}{\delta \tilde{\bar{K}}_{a}(-p)}\right]_{p h}+ \\
& +\int \frac{\mathrm{d}^{4} p}{(2 \pi)^{4}} \frac{\delta I_{0}^{G I}}{\delta \tilde{\phi}^{i}(p)}\left[\frac{\delta}{\delta \tilde{\omega}^{\gamma}(l)} \frac{\delta \Gamma^{(1)}}{\delta \tilde{K}_{i}(-p)}\right]_{p h}=0
\end{aligned}
$$

(the sum $\mathcal{T}_{\gamma} \varphi+\bar{P}_{\gamma}$ appearing in (2.12) has been replaced here by $\mathcal{T}_{\gamma} \varphi$, because the Stueckelberg fields, if present, are free in the Landau gauge - see eq. (A.7)).

As it is easy to realize (by looking at the Feynman rules), the last three terms of the left hand side of (5.3) vanish if the index $\gamma$ corresponds to an Abelian generator. The identity (5.3) takes then the form of the standard QED-like Ward-Takahashi (WT) identity. ${ }^{9}$

Taking functional derivatives of (5.3) w.r.t. "physical" fields and setting all fields to zero one obtains various Slavnov-Taylor (ST) identities. If the first term on the l.h.s., obtained as a result of differentiation of (5.3), is a 1PI function $X$, we call the resulting relation "the identity involving the $X$ function". At the one-loop order the 1PI functions related by a given ST identity receive contributions from bare one-loop diagrams, ${ }^{10}$ from minimal counterterms and from non-minimal ones. The strategy which we follow below is to take a ST identity and compute first the contributions (marked by the superscript $(1 B)$ ) of regularized bare one-loop diagrams. Because the regularization (3.1) (" $\Lambda$ Reg") breaks the BRST invariance, these contributions to the ST identity do not sum up to zero, but according to the QAP their sum, denoted $\Omega$ with appropriate indices, should be local in the infinite cutoff limit. This can be verified by doing more or less standard manipulations on regularized integrals. Since the calculations are rather lengthy, we do not show their details except for one case: in $\mathrm{C}$ we outline the steps necessary to work out the contribution of bare one-loop fermionic diagrams to the identity involving the $\langle A A A A\rangle$ function. The functions $\Omega$ obtained in this way represent one-loop breakings of the respective ST identities and have the obvious interpretation of appropriate derivatives w.r.t. to fields of the functional $\Omega_{0}$ defined in (4.4).

The next step is to take into account minimal counterterms specified by the prescription (4.12). The resulting one-loop breaking factors $\tilde{\Omega}$ with appropriate indices are just the appropriate derivatives w.r.t. to fields of the functional $\tilde{\Omega}_{0}$ defined in (4.15). From (4.16) and (4.15) it follows that obtaining $\tilde{\Omega}$ 's reduces to setting to zero in the corresponding $\Omega$ 's all factors $\delta_{\Lambda}$ defined in (4.13) and all terms proportional to $\Lambda^{2}$. (In fact, the universality of one-loop logarithmic divergences makes it clear that factors $\delta_{\Lambda}$ cannot appear in $\Omega$ 's and to obtain $\tilde{\Omega}$ 's it is enough to set quadratic divergences to zero in the corresponding $\Omega$ 's).

\footnotetext{
${ }^{9}$ Thus, Abelian ideals do not have to be considered separately — relevant constraints are already contained in the identity (5.3). This statement generalizes to higher orders, because the regularization (3.1) automatically preserves the Abelian antighost equation [45], see also eq. (A.6), in the infinite cutoff limit. In particular, Abelian WT identities follow from the ZJ identity (4.1) as a consequence of the algebraic relation (A.9).

${ }^{10}$ As there is no one-loop contribution to the function $\left\langle K_{i} \omega^{\gamma}\right\rangle$, the last term of (5.3) does not contribute if all differentiations act on the $I_{0}^{G I}$ factor. For this reason and because non-minimal counterterms are not allowed for this function (cf. (4.27)), all terms with $\left\langle K_{i} \omega^{\gamma}\right\rangle$ are omitted in the formulae below.
} 
The last step is the determination of the non-minimal counterterms, which in principle means solving eq. (4.17) with the auxiliary condition (4.27). Before presenting the systematic of this procedure, we remark that there is an alternative way of obtaining the necessary one-loop breaking factors $\Omega$. It relies on the fact that the bare 1PI functions $\widetilde{\Gamma}_{\text {Dim }}^{(1 B)}$ calculated using DimReg do satisfy the ST identities, provided the naive definition of $\gamma^{5}$ is employed. ${ }^{11}$ Thus, replacing in the ST identities each bare one-loop 1PI function $\widetilde{\Gamma}^{(1 B)} \equiv \widetilde{\Gamma}_{\Lambda}^{(1 B)}$ calculated in our regularization (3.1) by the difference

$$
\Delta \widetilde{\Gamma}^{(1 B)} \equiv \widetilde{\Gamma}^{(1 B)}-\widetilde{\Gamma}_{\operatorname{Dim}}^{(1 B)},
$$

must produce the same factors $\Omega$. The necessary differences $\Delta \widetilde{\Gamma}^{(1 B)}$, which will also be used in sections 6 and 7 to derive the two-loop RGE satisfied by the renormalized parameters in our scheme are calculated in B. This approach is obviously much simpler than the direct calculations in $\Lambda$ Reg, firstly, because the differences (5.4) are already local expressions ${ }^{12}$ and, secondly, because in this method the only 1PI function with antifields that contributes to the factors $\Omega$ is $\left\langle K_{\alpha}^{\mu} \omega^{\beta}\right\rangle$ (the corresponding difference is given in (B.5)); the remaining functions with antifields are the same in DimReg and $\Lambda$ Reg (even though the degree of divergence may indicate otherwise) due to the additional "symmetry" (A.5) of $\mathcal{L}_{0}^{\text {Rest }}(2.10)$ in the Landau gauge, which is preserved by both regularizations.

We stress however that, except for the bosonic contribution to the identity involving the $\langle A A A A\rangle$ function, all factors $\Omega$ have been computed directly in $\Lambda$ Reg (along the lines described in $\mathrm{C}$ ) and the results are, therefore, unaffected by ambiguities of DimReg with the naive prescription for $\gamma^{5}$.

Systematic determination of non-minimal counterterms restoring the BRST symmetry consists of considering first those ST identities in which only one 1PI function can have such a counterterm (this is established by inspection of the allowed set (4.27) of non-minimal counterterms) and moving successively to those in which more functions can have nonminimal counterterms but only one such counterterm which has not been determined yet. We have divided these steps into separate subsections. ${ }^{13}$

\subsection{Identity involving the $\langle\psi \psi A\rangle$ function}

Functionally differentiating (5.3) twice w.r.t. the Majorana fields one obtains the identity (we use the notation explained in $(2.14)-(2.15)$ )

$$
\begin{aligned}
& i l_{\mu} \widetilde{\Gamma}_{b_{1} b_{2} \gamma}^{\mu}\left(k_{1}, k_{2}, l\right)^{(1)}+t_{\gamma b_{1}}^{a} \widetilde{\Gamma}_{a b_{2}}\left(k_{1}+l, k_{2}\right)^{(1)}+\widetilde{\Gamma}_{b_{1} a}\left(k_{1}, k_{2}+l\right)^{(1)} t_{\gamma b_{2}}^{a}+ \\
& +\left(\mathcal{T}_{\gamma} \varphi\right)^{n} \widetilde{\Gamma}_{b_{1} b_{2} n}\left(k_{1}, k_{2}, l\right)^{(1)}-\mathcal{V}_{n}^{\prime}(\varphi)\left\langle\tilde{K}_{n}(0) \tilde{\psi}^{b_{1}}\left(k_{1}\right) \tilde{\psi}^{b_{2}}\left(k_{2}\right) \tilde{\omega}^{\gamma}(l)\right\rangle_{\widetilde{\mathrm{PI}}}^{(1)}+
\end{aligned}
$$

\footnotetext{
${ }^{11}$ Terms which are ambiguous due to using the anticommuting $\gamma^{5}$ in $d$-dimensions vanish if the condition (4.10) is fulfilled.

${ }^{12}$ Strictly speaking, functions on the r.h.s. of (5.4) depend on two different sets of couplings, say, $\left\{g^{C}\right\}$ and $\left\{\check{g}^{C}\right\}$. However, as will be shown in the next section, $g^{C}-\check{g}^{C}=\mathcal{O}(\hbar)$ and thus the resulting non-localities are of $\mathcal{O}\left(\hbar^{2}\right)$ order. Similarly, we assume here that non-local terms of order of $\mathcal{O}\left(\Lambda^{-1}\right)$ (or $\left.\mathcal{O}(d-4)\right)$ are neglected.

${ }^{13}$ Since minimal counterterms can be immediately obtained from divergent parts of formulae listed in $B$ we do not give them explicitly here; those needed for the calculation of the $\mathcal{O}\left(\hbar^{2}\right)$ vacuum graphs are given in section 8 .
} 


$$
\begin{aligned}
& +i\left(C \gamma^{\mu} t_{\alpha}\right)_{b_{1} b_{2}}\left\langle\tilde{K}_{\alpha}^{\mu}\left(k_{1}+k_{2}\right) \tilde{\omega}^{\gamma}(l)\right\rangle_{\widetilde{1 P I}}^{(1)}-\left[C\left(k_{1}-m_{F}\right)\right]_{a b_{1}}\left\langle\tilde{\bar{K}}_{a}\left(k_{1}\right) \tilde{\omega}^{\gamma}(l) \tilde{\psi}^{b_{2}}\left(k_{2}\right)\right\rangle_{\widetilde{1 P I}}^{(1)}+ \\
& +\left[C\left(k_{2}-m_{F}\right)\right]_{a b_{2}}\left\langle\tilde{\bar{K}}_{a}\left(k_{2}\right) \tilde{\omega}^{\gamma}(l) \tilde{\psi}^{b_{1}}\left(k_{1}\right)\right\rangle_{\widetilde{1 P I}}^{(1)}=0
\end{aligned}
$$

The contribution $\Omega_{b_{1} b_{2} \gamma}\left(k_{1}, k_{2}, l\right)$ of the bare one-loop diagrams to the l.h.s. of (5.5) (in the limit of infinite cutoff) is

$$
\begin{aligned}
\Omega_{b_{1} b_{2} \gamma}\left(k_{1}, k_{2}, l\right)=\frac{1}{2(4 \pi)^{2}}\left\{C l_{\mu} \gamma^{\mu}\right. & {\left[t_{\epsilon} \epsilon^{\epsilon} t_{\gamma}+\frac{3}{2}\left(1-\ln \frac{3}{4}\right) e^{\kappa}{ }_{\epsilon \beta} e_{\gamma}^{\epsilon \beta} t_{\kappa}+y_{i}^{*} y_{j} \mathcal{T}_{\gamma}^{i j}+\right.} \\
& \left.\left.-\left(\frac{1}{3}+\ln \frac{3}{4}\right) y_{i}^{*} y^{i} t_{\gamma}\right]\right\}_{b_{1} b_{2}} .
\end{aligned}
$$

Since $\Omega_{b_{1} b_{2} \gamma}\left(k_{1}, k_{2}, l\right)$ turns out to be $\Lambda$-independent, it is just equal $\tilde{\Omega}_{b_{1} b_{2} \gamma}\left(k_{1}, k_{2}, l\right)$ (notice that none of the 1PI functions involved is quadratically divergent). Inspection of (4.27) reveals that only the vertex $\langle\psi \psi A\rangle$ can have a non-minimal counterterm, of the general form

$$
\delta \widetilde{\Gamma}_{b_{1} b_{2} \gamma}^{\mu}\left(k_{1}, k_{2}, l\right)=i\left(C \gamma^{\mu} \delta^{b} \widehat{\mathscr{X}}_{F \gamma}\right)_{b_{1} b_{2}},
$$

(for simplicity we write $\delta \Gamma$ rather than $\delta_{b} \Gamma_{0}^{(1)}$ from now on). The equation

$$
\tilde{\Omega}_{b_{1} b_{2} \gamma}\left(k_{1}, k_{2}, l\right)+i l_{\mu} \delta \widetilde{\Gamma}_{b_{1} b_{2} \gamma}^{\mu}\left(k_{1}, k_{2}, l\right)=0
$$

necessary for fulfilling the ST identity (5.5) has the unique solution

$$
\begin{aligned}
\delta^{b} \widehat{\mathscr{X}}_{F \gamma}=\frac{1}{2(4 \pi)^{2}}[ & t_{\alpha} t^{\alpha} t_{\gamma}+\frac{3}{2}\left(1-\ln \frac{3}{4}\right) e^{\kappa}{ }_{\alpha \beta} e^{\alpha \beta}{ }_{\gamma} t_{\kappa}+y_{i}^{*} y_{j} \mathcal{T}_{\gamma}^{i j}+ \\
& \left.-\left(\frac{1}{3}+\ln \frac{3}{4}\right) y_{i}^{*} y^{i} t_{\gamma}\right] .
\end{aligned}
$$

\subsection{Identity involving the $\langle\phi A\rangle$ function}

Functional differentiation of (5.3) w.r.t. the scalar field yields the ST identity

$$
\begin{aligned}
& i l_{\mu} \widetilde{\Gamma}_{i \alpha}^{\mu}(p, l)^{(1)}+\mathcal{T}_{\alpha i}^{j} \widetilde{\Gamma}_{j}(p+l)^{(1)}+\left(\mathcal{T}_{\alpha} \varphi\right)^{j} \widetilde{\Gamma}_{i j}(p, l)^{(1)}+ \\
& \quad-\mathcal{V}_{j}^{\prime}(\varphi)\left\langle\tilde{K}_{j}(0) \tilde{\phi}^{i}(p) \tilde{\omega}^{\alpha}(l)\right\rangle_{\widetilde{1 \mathrm{PI}}}^{(1)}-i p^{\mu}\left(\mathcal{T}_{\gamma} \varphi\right)_{i}\left\langle\tilde{K}_{\gamma}^{\mu}(-l) \tilde{\omega}^{\alpha}(l)\right\rangle_{\widetilde{1 \mathrm{PI}}}^{(1)}=0 .
\end{aligned}
$$

The contribution $\Omega_{i \alpha}(p, l)$ of bare one-loop diagrams reads

$$
\begin{aligned}
\Omega_{i \alpha}(p, l)=\frac{l^{2}}{(4 \pi)^{2}}\{ & \left(\frac{7}{12}+\ln \frac{3}{2}\right) \operatorname{tr}\left[Y_{i} \mathfrak{f}_{\alpha} m_{F}^{*}-Y_{i}^{*} m_{F} \mathfrak{f}_{\alpha}\right]+ \\
& \left.+\frac{3}{4} \ln \frac{3}{4}\left(\varphi^{\mathrm{T}} \mathcal{T}^{\kappa} \mathcal{T}_{\alpha} \mathcal{T}_{\kappa}\right)_{i}-\frac{3}{4} \ln \frac{3}{2}\left(\varphi^{\mathrm{T}} \mathcal{T}_{\alpha} \mathcal{T}^{\kappa} \mathcal{T}_{\kappa}\right)_{i}\right\} .
\end{aligned}
$$

Again, since $\Omega_{i \alpha}^{\text {div }}(p, l)=0, \tilde{\Omega}_{i \alpha}(p, l)=\Omega_{i \alpha}(p, l)$. Only the $\langle\phi A\rangle$ function can have a non-minimal counterterm of the form

$$
\delta \widetilde{\Gamma}_{i \alpha}^{\mu}(p,-p)=i p^{\mu} \delta^{b} c_{i \alpha}
$$


in which $\delta^{b} c_{i \alpha}$ is a constant matrix. The Slavnov-Taylor identity (5.9) requires

$$
\tilde{\Omega}_{i \alpha}(p,-p)-i p_{\mu} \delta \widetilde{\Gamma}_{i \alpha}^{\mu}(p,-p)=0,
$$

whose unique solution is

$$
\begin{aligned}
\delta^{b} c_{i \alpha}=-\frac{1}{(4 \pi)^{2}}\{ & \left(\frac{7}{12}+\ln \frac{3}{2}\right) \operatorname{tr}\left[Y_{i} \mathfrak{f}_{\alpha} m_{F}^{*}-Y_{i}^{*} m_{F} \mathfrak{f}_{\alpha}\right]+ \\
& \left.+\frac{3}{4} \ln \frac{3}{4}\left(\varphi^{\mathrm{T}} \mathcal{T}^{\kappa} \mathcal{T}_{\alpha} \mathcal{T}_{\kappa}\right)_{i}-\frac{3}{4} \ln \frac{3}{2}\left(\varphi^{\mathrm{T}} \mathcal{T}_{\alpha} \mathcal{T}^{\kappa} \mathcal{T}_{\kappa}\right)_{i}\right\} .
\end{aligned}
$$

\subsection{Identity involving the $\langle A A\rangle$ function}

The identity involving the vacuum polarization tensor reads

$$
\begin{aligned}
& i l_{\mu} \widetilde{\Gamma}_{\alpha \beta}^{\mu \nu}(l, p)^{(1)}+\left(\mathcal{T}_{\alpha} \varphi\right)^{i} \widetilde{\Gamma}_{i \beta}^{\nu}(l, p)^{(1)}-\mathcal{V}_{i}^{\prime}(\varphi)\left\langle\tilde{K}_{i}(0) \tilde{\omega}^{\alpha}(l) \tilde{A}_{\nu}^{\beta}(p)\right\rangle_{\widetilde{1 \mathrm{PI}}}^{(1)}+ \\
& +\left\{m_{V \beta \kappa}^{2} \eta^{\nu \sigma}+\delta_{\beta \kappa}\left(p^{\nu} p^{\sigma}-\eta^{\nu \sigma} p^{2}\right)\right\}\left\langle\tilde{K}_{\kappa}^{\sigma}(p) \tilde{\omega}^{\alpha}(l)\right\rangle_{\widetilde{1 \mathrm{PI}}}^{(1)}=0 .
\end{aligned}
$$

The breaking $\Omega_{\alpha \beta}^{\nu}(l, p)$ calculated directly from the bare one-loop diagrams has the form

$$
\Omega_{\alpha \beta}^{\nu}(l, p)=i l^{\nu} \frac{1}{(4 \pi)^{2}} \mathbb{W}_{\alpha \beta}(l, p),
$$

where

$$
\begin{aligned}
\mathbb{W}_{\alpha \beta}(l, p)=\left\{\Lambda^{2}+\frac{l^{2}}{3}\right\} \operatorname{tr}\left[\mathfrak{f}_{\alpha} \mathfrak{f}_{\beta}\right]-\left\{\frac{1}{3}+\ln \frac{3}{4}\right\} \operatorname{tr}\left[\left\{\mathfrak{f}_{\alpha}, \mathfrak{f}_{\beta}\right\} m_{F}^{*} m_{F}\right]+ \\
-\left\{\frac{1}{3}-2 \ln \frac{3}{4}\right\} \operatorname{tr}\left[\mathfrak{f}_{\alpha} m_{F}^{*} \mathfrak{f}_{\beta}^{*} m_{F}\right]-\left\{\frac{\Lambda^{2}}{2}+\frac{5}{48} l^{2}\right\} \operatorname{tr}\left[\mathcal{T}_{\alpha} \mathcal{T}_{\beta}\right]+\frac{1}{2} \operatorname{tr}\left[m_{S}^{2} \mathcal{T}_{\alpha} \mathcal{T}_{\beta}\right]+ \\
\quad-\frac{3}{4} \ln \frac{3}{4} \varphi^{\mathrm{T}}\left\{\mathcal{T}_{\beta}, \mathcal{T}^{\kappa}\right\} \mathcal{T}_{\alpha} \mathcal{T}_{\kappa} \varphi-\left\{\Lambda^{2}+\frac{5}{24} l^{2}\right\} \operatorname{tr}\left[e_{\alpha} e_{\beta}\right]+ \\
\quad+\frac{3}{4}\left\{2-\ln \frac{3}{4}\right\} \operatorname{tr}\left[m_{V}^{2} e_{\alpha} e_{\beta}\right] .
\end{aligned}
$$

Taking into account minimal counterterms (i.e. setting $\Lambda^{2}$ to zero in $\Omega_{\alpha \beta}^{\nu}(l, p)$ ) yields $\tilde{\Omega}_{\alpha \beta}^{\nu}(l, p)$. Comparison of (5.14) with (4.27) reveals that two non-minimal counterterms can contribute to (5.14): the already determined counterterm (5.11) (contributing to the $\langle\phi A\rangle$ function) and the one for the vacuum polarization which must be of the general form

$$
\delta \widetilde{\Gamma}_{\beta \kappa}^{\nu \rho}(p,-p)=\eta^{\nu \rho}\left(\delta^{b} m_{V}^{2}\right)_{\beta \kappa}+p^{\nu} p^{\rho}\left(\delta^{b} z_{A}\right)_{\beta \kappa},
$$

with symmetric matrices $\delta^{b} m_{V}^{2}$ and $\delta^{b} z_{A}$. Fulfilling the identity (5.14) requires that

$$
\tilde{\Omega}_{\alpha \beta}^{\nu}(l,-l)+i l_{\mu} \delta \widetilde{\Gamma}_{\alpha \beta}^{\mu \nu}(l,-l)+\left(\mathcal{T}_{\alpha} \varphi\right)^{i} \delta \widetilde{\Gamma}_{i \beta}^{\nu}(l,-l)=0 .
$$

Using the explicit form (5.13) of $\delta \widetilde{\Gamma}_{i \beta}^{\nu}(l,-l)$ one finds the unique solution:

$$
\left(\delta^{b} z_{A}\right)_{\alpha \beta}=\frac{1}{(4 \pi)^{2}}\left\{-\frac{1}{3} \operatorname{tr}\left[\mathfrak{f}_{\alpha} \mathfrak{f}_{\beta}\right]+\frac{5}{48} \operatorname{tr}\left[\mathcal{T}_{\alpha} \mathcal{T}_{\beta}\right]+\frac{5}{24} \operatorname{tr}\left[e_{\alpha} e_{\beta}\right]\right\},
$$


and

$$
\begin{aligned}
(4 \pi)^{2}\left(\delta^{b} m_{V}^{2}\right)_{\alpha \beta}= & -\left\{\frac{1}{4}+\ln 2\right\} \operatorname{tr}\left[\left\{\mathfrak{f}_{\alpha}, \mathfrak{f}_{\beta}\right\} m_{F}^{*} m_{F}\right]+ \\
& +\left\{\frac{3}{2}+2 \ln 2\right\} \operatorname{tr}\left[\mathfrak{f}_{\alpha} m_{F}^{*} \mathfrak{f}_{\beta}^{*} m_{F}\right]-\frac{1}{2} \operatorname{tr}\left[m_{S}^{2} \mathcal{T}_{\alpha} \mathcal{T}_{\beta}\right]+ \\
& -\frac{3}{4}\left\{2-\ln \frac{3}{4}\right\} \operatorname{tr}\left[m_{V}^{2} e_{\alpha} e_{\beta}\right]+\frac{3}{4} \ln \frac{3}{4} \varphi^{\mathrm{T}}\left\{\mathcal{T}_{\beta}, \mathcal{T}^{\kappa}\right\} \mathcal{T}_{\alpha} \mathcal{T}_{\kappa} \varphi+ \\
& +\frac{3}{4} \ln \frac{3}{4} \varphi^{\mathrm{T}} \mathcal{T}^{\kappa} \mathcal{T}_{\beta} \mathcal{T}_{\kappa} \mathcal{T}_{\alpha} \varphi-\frac{3}{4} \ln \frac{3}{2} \varphi^{\mathrm{T}} \mathcal{T}_{\beta} \mathcal{T}^{\kappa} \mathcal{T}_{\kappa} \mathcal{T}_{\alpha} \varphi .
\end{aligned}
$$

\subsection{Identity involving the $\langle\phi \phi A\rangle$ function}

The ST identity involving the $\widetilde{\Gamma}_{i j \alpha}^{\mu}$ vertex has the form

$$
\begin{aligned}
& i l_{\mu} \widetilde{\Gamma}_{i j \alpha}^{\mu}\left(p, p^{\prime}, l\right)^{(1)}+\mathcal{T}_{\alpha i}^{n} \widetilde{\Gamma}_{n j}\left(p+l, p^{\prime}\right)^{(1)}+\widetilde{\Gamma}_{\mathrm{in}}\left(p, p^{\prime}+l\right)^{(1)} \mathcal{T}_{\alpha j}^{n}+ \\
& +\left(\mathcal{T}_{\alpha} \varphi\right)^{n} \widetilde{\Gamma}_{i j n}\left(p, p^{\prime}, l\right)^{(1)}-\mathcal{V}_{n}^{\prime}(\varphi)\left\langle\tilde{K}_{n}(0) \tilde{\phi}^{i}(p) \tilde{\phi}^{j}\left(p^{\prime}\right) \tilde{\omega}^{\alpha}(l)\right\rangle_{\widetilde{1 \mathrm{PI}}}^{(1)}+ \\
& -i p^{\prime \mu}\left(\mathcal{T}_{\gamma} \varphi\right)_{j}\left\langle\tilde{\phi}^{i}(p) \tilde{K}_{\gamma}^{\mu}\left(p^{\prime}\right) \tilde{\omega}^{\alpha}(l)\right\rangle_{\widetilde{1 \mathrm{PI}}}^{(1)}-i p^{\mu}\left(\mathcal{T}_{\gamma} \varphi\right)_{i}\left\langle\tilde{\phi}^{j}\left(p^{\prime}\right) \tilde{K}_{\gamma}^{\mu}(p) \tilde{\omega}^{\alpha}(l)\right\rangle_{\widetilde{1 \mathrm{PI}}}^{(1)}+ \\
& +\left(p^{2}-m_{S}^{2}\right)_{\mathrm{in}}\left\langle\tilde{K}_{n}(p) \tilde{\phi}^{j}\left(p^{\prime}\right) \tilde{\omega}^{\alpha}(l)\right\rangle_{\widetilde{\mathrm{PPI}}}^{(1)}+\left(p^{\prime 2}-m_{S}^{2}\right)_{j n}\left\langle\tilde{K}_{n}\left(p^{\prime}\right) \tilde{\phi}^{i}(p) \tilde{\omega}^{\alpha}(l)\right\rangle_{\widetilde{\mathrm{PI}}}^{(1)}+ \\
& +i\left(p-p^{\prime}\right)^{\mu} \mathcal{T}_{\gamma j i}\left\langle\tilde{K}_{\gamma}^{\mu}\left(p+p^{\prime}\right) \tilde{\omega}^{\alpha}(l)\right\rangle_{\widetilde{\mathrm{PI}}}^{(1)}=0 .
\end{aligned}
$$

The contribution of purely one-loop diagrams to the 1.h.s. of (5.21) in the limit $\Lambda \rightarrow \infty$ is finite and reads

$$
\begin{aligned}
\tilde{\Omega}_{i j \alpha}\left(p, p^{\prime}, l\right)=\frac{1}{(4 \pi)^{2}}\left(p^{2}-p^{\prime 2}\right)\{ & \left(\frac{7}{12}+\ln \frac{3}{2}\right) \operatorname{tr}\left[Y_{i} \mathfrak{f}_{\alpha} Y_{j}^{*}-Y_{i}^{*} Y_{j} \mathfrak{f}_{\alpha}\right]+ \\
+ & \left.\frac{3}{4}\left[\ln 2\left(\mathcal{T}^{\kappa} \mathcal{T}_{\alpha} \mathcal{T}_{\kappa}\right)_{i j}-\ln \frac{3}{2} e^{\kappa \delta}\left(\mathcal{T}_{\kappa} \mathcal{T}_{\delta}\right)_{i j}\right]\right\}
\end{aligned}
$$

In agreement with the expectation $\tilde{\Omega}_{i j \alpha}\left(p, p^{\prime}, l\right)$ is related to $(5.10)$ by

$$
\tilde{\Omega}_{i j \alpha}(p, 0,-p)=\frac{\partial}{\partial \varphi^{j}} \tilde{\Omega}_{i \alpha}(p,-p) .
$$

According to (4.27) only the function $\langle\phi \phi A\rangle$ can have a non-minimal counterterm. Its form

$$
\delta \widetilde{\Gamma}_{i j \alpha}^{\mu}\left(p, p^{\prime}, l\right)=i\left(p^{\mu} \delta^{b} \mathscr{X}_{\alpha j i}+p^{\prime \mu} \delta^{b} \mathscr{X}_{\alpha i j}\right),
$$

with an arbitrary constant tensor $\delta^{b} \mathscr{X}_{\alpha j i}$ is dictated by the requirement of the Bose-Einstein statistics. Fulfillment of (5.21) imposes the condition

$$
\tilde{\Omega}_{i j \alpha}\left(p, p^{\prime}, l\right)+i l_{\mu} \delta \widetilde{\Gamma}_{i j \alpha}^{\mu}\left(p, p^{\prime}, l\right)=0,
$$

and the unique solution is the tensor

$$
\begin{aligned}
\delta^{b} \mathscr{X}_{\alpha i j}=\frac{1}{(4 \pi)^{2}}\{ & \left(\frac{7}{12}+\ln \frac{3}{2}\right) \operatorname{tr}\left[Y_{i} \mathfrak{f}_{\alpha} Y_{j}^{*}-Y_{i}^{*} Y_{j} \mathfrak{f}_{\alpha}\right]+ \\
& \left.+\frac{3}{4}\left[\ln 2\left(\mathcal{T}^{\kappa} \mathcal{T}_{\alpha} \mathcal{T}_{\kappa}\right)_{i j}-\ln \frac{3}{2} e^{\kappa \delta}\left(\mathcal{T}_{\kappa} \mathcal{T}_{\delta}\right)_{i j}\right]\right\},
\end{aligned}
$$


which is antisymmetric in the $i j$ indices. It is related to the counterterm (5.13) by

$$
\delta^{b} \mathscr{X}_{\alpha j i}=\frac{\partial}{\partial \varphi^{j}} \delta^{b} c_{i \alpha}
$$

\subsection{Identities involving the $\langle\phi A A\rangle$ and $\langle\phi \phi A A\rangle$ functions}

The relations (5.23) and (5.27) reflect two facts: the preservation by our regularization prescription of the shift symmetry (3.3) and that the same requirement has been imposed on non-minimal counterterms in section 4 (see also appendix A.2). Therefore the nonminimal counterterms for the $\langle\phi A A\rangle$ and $\langle\phi \phi A A\rangle$ 1PI functions must be given by (power counting implies they are momentum independent)

$$
\delta \widetilde{\Gamma}_{i \beta \kappa}^{\nu \rho}\left(l, p, p^{\prime}\right)=\delta \widetilde{\Gamma}_{i \beta \kappa}^{\nu \rho}(0,0,0)=\frac{\partial}{\partial \varphi^{i}} \delta \widetilde{\Gamma}_{\beta \kappa}^{\nu \rho}(0,0)=\eta^{\nu \rho} \frac{\partial}{\partial \varphi^{i}}\left(\delta^{b} m_{V}^{2}\right)_{\beta \kappa},
$$

and

$$
\delta \widetilde{\Gamma}_{i j \beta \kappa}^{\nu \rho}\left(l, l^{\prime}, p, p^{\prime}\right)=\frac{\partial^{2}}{\partial \varphi^{i} \partial \varphi^{j}} \delta \widetilde{\Gamma}_{\beta \kappa}^{\nu \rho}(0,0)=\eta^{\nu \rho} \frac{\partial^{2}}{\partial \varphi^{i} \partial \varphi^{j}}\left(\delta^{b} m_{V}^{2}\right)_{\beta \kappa} .
$$

The matrix $\left(\delta^{b} m_{V}^{2}\right)_{\beta \kappa}$ is given in eq. (5.20).

\subsection{Identity involving the $\langle A A A\rangle$ function}

Because of its relation to anomalies, one of the most interesting is the ST identity involving the triple vector boson vertex $\widetilde{\Gamma}_{\alpha \beta \gamma}^{\mu \nu \rho}\left(l, p, p^{\prime}\right)^{(1)}$

$$
\begin{aligned}
& i l_{\mu} \widetilde{\Gamma}_{\alpha \beta \gamma}^{\mu \nu \rho}\left(l, p, p^{\prime}\right)^{(1)}+e^{\kappa}{ }_{\alpha \beta} \widetilde{\Gamma}_{\kappa \gamma}^{\nu \rho}\left(p+l, p^{\prime}\right)^{(1)}+e^{\kappa}{ }_{\alpha \gamma} \widetilde{\Gamma}_{\kappa \beta}^{\rho \nu}\left(p^{\prime}+l, p\right)^{(1)}+ \\
& +\left(\mathcal{T}_{\alpha} \varphi\right)^{i} \widetilde{\Gamma}_{i \beta \gamma}^{\nu \rho}\left(l, p, p^{\prime}\right)^{(1)}-\mathcal{V}_{i}^{\prime}(\varphi)\left\langle\tilde{K}_{i}(0) \tilde{\omega}^{\alpha}(l) \tilde{A}_{\nu}^{\beta}(p) \tilde{A}_{\rho}^{\gamma}\left(p^{\prime}\right)\right\rangle_{\widetilde{1 P I}}^{(1)}+ \\
& +i p^{\rho}\left(\mathcal{T}_{\gamma} \varphi\right)_{i}\left\langle\tilde{K}_{i}\left(p^{\prime}\right) \tilde{\omega}^{\alpha}(l) \tilde{A}_{\nu}^{\beta}(p)\right\rangle_{\widetilde{1 P I}}^{(1)}+i p^{\nu}\left(\mathcal{T}_{\beta} \varphi\right)_{i}\left\langle\tilde{K}_{i}(p) \tilde{\omega}^{\alpha}(l) \tilde{A}_{\rho}^{\gamma}\left(p^{\prime}\right)\right\rangle_{\widetilde{1 \mathrm{PI}}}^{(1)}+ \\
& +\left\{m_{V \gamma \kappa}^{2} \eta^{\rho \sigma}+\delta_{\gamma \kappa}\left(p^{\prime \rho} p^{\prime \sigma}-\eta^{\rho \sigma} p^{\prime 2}\right)\right\}\left\langle\tilde{K}_{\kappa}^{\sigma}\left(p^{\prime}\right) \tilde{\omega}^{\alpha}(l) \tilde{A}_{\nu}^{\beta}(p)\right\rangle_{\widetilde{1 \mathrm{PI}}}^{(1)}+ \\
& +\left\{m_{V \beta \kappa}^{2} \eta^{\nu \sigma}+\delta_{\beta \kappa}\left(p^{\nu} p^{\sigma}-\eta^{\nu \sigma} p^{2}\right)\right\}\left\langle\tilde{K}_{\kappa}^{\sigma}(p) \tilde{\omega}^{\alpha}(l) \tilde{A}_{\rho}^{\gamma}\left(p^{\prime}\right)\right\rangle_{\widetilde{1 \mathrm{PI}}}^{(1)}+ \\
& +i e_{\gamma \beta \kappa}\left\{\left(p-p^{\prime}\right)^{\sigma} \eta^{\rho \nu}+\left(2 p^{\prime}+p\right)^{\nu} \eta^{\rho \sigma}-\left(2 p+p^{\prime}\right)^{\rho} \eta^{\nu \sigma}\right\}\left\langle\tilde{K}_{\kappa}^{\sigma}\left(p+p^{\prime}\right) \tilde{\omega}^{\alpha}(l)\right\rangle_{\widetilde{1 \mathrm{PI}}}^{(1)}=0 .
\end{aligned}
$$


Contribution $\Omega_{\alpha \beta \gamma}^{\nu \rho}\left(l, p, p^{\prime}\right)$ of bare one-loop diagrams to the left hand side of (5.30) obtained by direct manipulation of regularized integrals is

$$
\begin{aligned}
\Omega_{\alpha \beta \gamma}^{\nu \rho}\left(l, p, p^{\prime}\right)= & \Omega_{\alpha \beta \gamma}^{\nu \rho}\left(l, p, p^{\prime}\right)^{\text {anom }}+ \\
& +\frac{1}{6(4 \pi)^{2}} \operatorname{tr}\left(\left[\mathcal{T}_{\gamma}, \mathcal{T}_{\beta}\right] \mathcal{T}_{\alpha}\right) \times \\
& \quad \times\left\{\left(p^{2}-p^{\prime 2}\right) \eta^{\nu \rho}\left(\frac{19}{24}+\ln \frac{3}{2}\right)-\left(p^{\rho} p^{\nu}-p^{\prime \rho} p^{\prime \nu}\right)\left(\frac{17}{12}+\ln \frac{3}{2}\right)\right\} \\
& +\frac{1}{36(4 \pi)^{2}} \operatorname{tr}\left(e_{\gamma} e_{\alpha} e_{\beta}\right) \times\left\{\eta^{\nu \rho}\left(p^{2}-p^{\prime 2}\right)[24 \ln 2-6 \ln 3-19]+\right. \\
& \left.\left.+\frac{1}{18} \cdot \frac{1}{(4 \pi)^{2}} \operatorname{tr}\left(\mathfrak{f}_{\alpha}\left[\mathfrak{f}_{\beta}, \mathfrak{f}_{\gamma}\right]\right) \times p^{\prime \rho} p^{\prime \nu}\right)[24 \ln 2-6 \ln 3-34]\right\}+ \\
& \times\left[\left(1-12 \ln \frac{3}{2}\right)\left(p^{\prime \nu} p^{\prime \rho}-p^{\nu} p^{\rho}\right)-\eta^{\nu \rho}\left(5+12 \ln \frac{3}{2}\right)\left(p^{2}-p^{\prime 2}\right)\right]
\end{aligned}
$$

Once again $\tilde{\Omega}_{\alpha \beta \gamma}^{\nu \rho}\left(l, p, p^{\prime}\right)=\Omega_{\alpha \beta \gamma}^{\nu \rho}\left(l, p, p^{\prime}\right)$. The first term of (5.31) is the true anomaly which in our regularization has the form

$$
\Omega_{\alpha \beta \gamma}^{\nu \rho}\left(l, p, p^{\prime}\right)^{\text {anom }}=\frac{2 i}{3(4 \pi)^{2}} \operatorname{tr}\left(\mathfrak{f}_{\alpha}\left\{\mathfrak{f}_{\beta}, \mathfrak{f}_{\gamma}\right\}\right) \cdot p_{\sigma} p_{\tau}^{\prime} \epsilon^{\sigma \tau \nu \rho}, \quad \epsilon^{0123}=-1 .
$$

Except for this one, all the remaining terms of (5.31) can be also obtained (as already explained) by inserting in (5.30) the appropriate differences (5.4). The part of the $\langle A A A\rangle$ vertex that involves the Levi-Civita tensor is ambiguous ${ }^{14}$ in the DimReg with naive (anticommuting) $\gamma^{5}$ and therefore the term (5.32) can be obtained only directly in $\Lambda$ Reg; the calculation is similar to the one for the $\langle A A A A\rangle$ vertex which is outlined in $\mathrm{C}$ (we show there that the anomalies are independent of the shape of regularizing function in (3.1) as long as it satisfies the appropriate boundary conditions).

According to (4.27) non-minimal counterterms are allowed for the $\langle A A A\rangle,\langle A A\rangle$ and $\langle\phi A A\rangle$ vertices. The last two have already been determined (the formulae (5.17) and (5.28), respectively). It is well known that in general the metric-independent part of the counterterm to the $\langle A A A\rangle$ vertex converts only one form of the anomaly into another one but cannot remove it - the anomaly is cohomologically nontrivial. Therefore, we seek only a metric-dependent non-minimal counterterm. The most general form of such a counterterm (which takes into account the requirements of the Bose-Einstein statistics) reads

$$
\begin{aligned}
& \delta \widetilde{\Gamma}_{\alpha_{1} \alpha_{2} \alpha_{3}}^{\mu_{1} \mu_{2} \mu_{3}}\left(l_{1}, l_{2}, l_{3}\right)=-i\left\{\eta^{\mu_{1} \mu_{2}}\left[l_{1}^{\mu_{3}} \delta^{b} \mathrm{a}_{\alpha_{1} \alpha_{2} \alpha_{3}}+l_{2}^{\mu_{3}} \delta^{b} \mathrm{a}_{\alpha_{2} \alpha_{1} \alpha_{3}}\right]+\right. \\
& \left.\quad+\eta^{\mu_{1} \mu_{3}}\left[l_{1}^{\mu_{2}} \delta^{b_{a}} a_{\alpha_{1} \alpha_{3} \alpha_{2}}+l_{3}^{\mu_{2}} \delta^{b} \mathrm{a}_{\alpha_{3} \alpha_{1} \alpha_{2}}\right]+\eta^{\mu_{2} \mu_{3}}\left[l_{2}^{\mu_{1}} \delta^{b} \mathrm{a}_{\alpha_{2} \alpha_{3} \alpha_{1}}+l_{3}^{\mu_{1}} \delta^{b} \mathrm{a}_{\alpha_{3} \alpha_{2} \alpha_{1}}\right]\right\},
\end{aligned}
$$

with an arbitrary constant tensor $\delta^{b} a_{\alpha_{1} \alpha_{2} \alpha_{3}}$. The condition

$$
\begin{aligned}
& \tilde{\Omega}_{\alpha \beta \gamma}^{\nu \rho}\left(l, p, p^{\prime}\right)+i l_{\mu} \delta \widetilde{\Gamma}_{\alpha \beta \gamma}^{\mu \nu \rho}\left(l, p, p^{\prime}\right)+ \\
& \quad+e^{\kappa}{ }_{\alpha \beta} \delta \widetilde{\Gamma}_{\kappa \gamma}^{\nu \rho}\left(p+l, p^{\prime}\right)+e^{\kappa}{ }_{\alpha \gamma} \delta \widetilde{\Gamma}_{\kappa \beta}^{\rho \nu}\left(p^{\prime}+l, p\right)+\left(\mathcal{T}_{\alpha} \varphi\right)^{i} \delta \widetilde{\Gamma}_{i \beta \gamma}^{\nu \rho}\left(l, p, p^{\prime}\right)=0,
\end{aligned}
$$

\footnotetext{
${ }^{14}$ Ambiguous terms are multiplied by $\operatorname{tr}\left(\mathfrak{f}_{\alpha}\left\{\mathfrak{f}_{\beta}, \mathfrak{f}_{\gamma}\right\}\right)$ and thus vanish if the gauge group representation furnished by fermions is non-anomalous.
} 
has the unique solution (provided the condition (4.10) is fulfilled):

$$
\begin{aligned}
(4 \pi)^{2} \delta^{b} \mathrm{a}_{\alpha \beta \gamma}=e_{\alpha \beta}^{\kappa}\{ & -\frac{1}{18}\left(5+12 \ln \frac{3}{2}\right) \operatorname{tr}\left(\mathfrak{f}_{\gamma} \mathfrak{f}_{\kappa}\right)-\frac{1}{6}\left(\frac{19}{24}+\ln \frac{3}{2}\right) \operatorname{tr}\left(\mathcal{T}_{\gamma} \mathcal{T}_{\kappa}\right)+ \\
& \left.+\frac{1}{72}[24 \ln 2-6 \ln 3-19] \operatorname{tr}\left(e_{\gamma} e_{\kappa}\right)\right\}
\end{aligned}
$$

Owing to the total antisymmetry of $\delta^{\mathrm{b}} \mathrm{a}$, the counterterm (5.33) differs from the tree-level vertex (3.5) only by the replacement of structure constants with $\delta^{b}$ a.

\subsection{Identity involving the $\langle A A A A\rangle$ function}

The last non-minimal counterterm from the list (4.27) to be determined is the one for the four-vector boson vertex. To make the formulae simpler it is convenient to introduce the following notation

$$
\imath_{n} \equiv\left(\alpha_{n}, \mu_{n}, l_{n}\right), \quad \tilde{A}_{\imath_{n}} \equiv \tilde{A}_{\mu_{n}}^{\alpha_{n}}\left(l_{n}\right),
$$

and to define the operator $\mathbb{S}$ which symmetrizes expressions w.r.t. $\left(\imath_{2}, \imath_{3}, \imath_{4}\right)$ :

$$
\mathbb{S}\left\{F\left(\imath_{1}, \imath_{2}, \imath_{3}, \imath_{4}\right)\right\} \equiv \frac{1}{3 !} \sum_{\sigma \in S(\{2,3,4\})} F\left(\imath_{1}, \imath_{\sigma(2)}, \imath_{\sigma(3)}, \imath_{\sigma(4)}\right) .
$$

In this notation the relevant ST identity takes the form

$$
\begin{aligned}
& i\left(l_{1}\right)_{\mu_{1}} \widetilde{\Gamma}_{\alpha_{1} \alpha_{2} \alpha_{3} \alpha_{4}}^{\mu_{1} \mu_{2} \mu_{3} \mu_{4}}\left(l_{1}, l_{2}, l_{3}, l_{4}\right)^{(1)}+\left(\mathcal{T}_{\alpha_{1}} \varphi\right)^{j}\left\langle\tilde{\phi}^{j}\left(l_{1}\right) \tilde{A}_{l_{2}} \tilde{A}_{l_{3}} \tilde{A}_{l_{4}}\right\rangle_{\widetilde{1 P I}}^{(1)}+ \\
& +3 \mathbb{S}\left\{e_{\alpha_{1} \alpha_{2}}^{\kappa}\left\langle\tilde{A}_{\mu_{2}}^{\kappa}\left(l_{1}+l_{2}\right) \tilde{A}_{\imath_{3}} \tilde{A}_{\imath_{4}}\right\rangle_{\widetilde{1 P I}}^{(1)}\right\}+ \\
& +\left\langle\tilde{A}_{\rho}^{\kappa}\left(l_{1}\right) \tilde{A}_{\imath_{2}} \tilde{A}_{\imath_{3}} \tilde{A}_{\imath_{4}}\right\rangle_{\widetilde{1 \mathrm{PI}}}^{(0)}\left\langle\tilde{K}_{\kappa}^{\rho}\left(-l_{1}\right) \tilde{\omega}^{\alpha_{1}}\left(l_{1}\right)\right\rangle_{\widetilde{\mathrm{PI}}}^{(1)}+ \\
& +3 \mathbb{S}\left\{\left\langle\tilde{A}_{\rho}^{\kappa}\left(l_{1}+l_{4}\right) \tilde{A}_{\imath_{2}} \tilde{A}_{\imath_{3}}\right\rangle_{\widetilde{1 \mathrm{PI}}}^{(0)}\left\langle\tilde{K}_{\kappa}^{\rho}\left(-l_{1}-l_{4}\right) \tilde{\omega}^{\alpha_{1}}\left(l_{1}\right) \tilde{A}_{\imath_{4}}\right\rangle_{\widetilde{1 \mathrm{PI}}}^{(1)}\right\}+ \\
& +3 \mathbb{S}\left\{\left\langle\tilde{A}_{\rho}^{\kappa}\left(-l_{2}\right) \tilde{A}_{\imath_{2}}\right\rangle_{\widetilde{1 \mathrm{PI}}}^{(0)}\left\langle\tilde{K}_{\kappa}^{\rho}\left(l_{2}\right) \tilde{\omega}^{\alpha_{1}}\left(l_{1}\right) \tilde{A}_{\imath_{3}} \tilde{A}_{\imath_{4}}\right\rangle_{\widetilde{\mathrm{PI}}}^{(1)}\right\}+ \\
& +3 \mathbb{S}\left\{\left\langle\tilde{\phi}^{j}\left(-l_{2}\right) \tilde{A}_{\imath_{2}}\right\rangle_{\widetilde{\mathrm{PI}}}^{(0)}\left\langle\tilde{K}_{j}\left(l_{2}\right) \tilde{\omega}^{\alpha_{1}}\left(l_{1}\right) \tilde{A}_{\imath_{3}} \tilde{A}_{\imath_{4}}\right\rangle_{\widetilde{\mathrm{PPI}}}^{(1)}\right\}+ \\
& +3 \mathbb{S}\left\{\left\langle\tilde{\phi}^{j}\left(-l_{2}-l_{3}\right) \tilde{A}_{\imath_{2}} \tilde{A}_{\imath_{3}}\right\rangle_{\widetilde{1 \mathrm{PI}}}^{(0)}\left\langle\tilde{K}_{j}\left(l_{2}+l_{3}\right) \tilde{\omega}^{\alpha_{1}}\left(l_{1}\right) \tilde{A}_{\imath_{4}}\right\rangle_{\widetilde{1 P I}}^{(1)}\right\}+ \\
& -\mathcal{V}_{j}^{\prime}(\varphi)\left\langle\tilde{K}_{j}(0) \tilde{\omega}^{\alpha_{1}}\left(l_{1}\right) \tilde{A}_{\imath_{2}} \tilde{A}_{\imath_{3}} \tilde{A}_{\imath_{4}}\right\rangle_{\widetilde{1 \mathrm{PI}}}^{(1)}=0 .
\end{aligned}
$$

Power counting, Lorentz properties and the antighost equation (A.5) imply that in (5.38) only the functions $\langle A A A A\rangle,\langle A A A\rangle$, and $\left\langle K_{\kappa}^{\rho} \omega^{\alpha}\right\rangle$ can be different in $\Lambda$ Reg and DimReg. Therefore, $\Omega_{\alpha_{1} \alpha_{2} \alpha_{3} \alpha_{4}}^{\mu_{2} \mu_{3} \mu_{4}}\left(l_{1}, l_{2}, l_{3}, l_{4}\right)$ which is identical with $\tilde{\Omega}_{\alpha_{1} \alpha_{2} \alpha_{3} \alpha_{4}}^{\mu_{2} \mu_{3} \mu_{4}}\left(l_{1}, l_{2}, l_{3}, l_{4}\right)$ can be obtained using the differences (B.16), (B.7) and (B.5). ${ }^{15}$ As follows from (4.27), only the

\footnotetext{
${ }^{15}$ Unlike the previously considered identities, only the (potentially anomalous) contribution of fermions to (5.38) has been worked out directly in $\Lambda$ Reg (the calculation is outlined in $\mathrm{C}$ ). This contribution is correctly reproduced by the differences (B.16), (B.7) and (B.5) if (4.10) is satisfied.
} 
vertices $\langle A A A A\rangle$ and $\langle A A A\rangle$ have non-minimal counterterms; (5.38) requires therefore that

$$
\begin{gathered}
\tilde{\Omega}_{\alpha_{1} \alpha_{2} \alpha_{3} \alpha_{4}}^{\mu_{2} \mu_{3} \mu_{4}}\left(l_{1}, l_{2}, l_{3}, l_{4}\right)+3 \mathbb{S}\left\{e^{\kappa}{ }_{\alpha_{1} \alpha_{2}} \delta \widetilde{\Gamma}_{\kappa}^{\mu_{2} \mu_{3} \mu_{3} \mu_{4}}\left(l_{1}+l_{2}, l_{3}, l_{4}\right)\right\}+ \\
+i\left(l_{1}\right)_{\mu_{1}} \delta \widetilde{\Gamma}_{\alpha_{1} \alpha_{2} \alpha_{3} \alpha_{4}}^{\mu_{1} \mu_{2} \mu_{3} \mu_{4}}\left(l_{1}, l_{2}, l_{3}, l_{4}\right)=0 .
\end{gathered}
$$

The explicit form of $\tilde{\Omega}_{\alpha_{1} \alpha_{2} \alpha_{3} \alpha_{4}}^{\mu_{2} \mu_{3} \mu_{4}}\left(l_{1}, l_{2}, l_{3}, l_{4}\right)$ is rather complicated, however simplifications occur after combining it with the second term in which $\delta \widetilde{\Gamma}_{\kappa}^{\mu_{2} \mu_{3} \mu_{4} \alpha_{4}}\left(l_{1}+l_{2}, l_{3}, l_{4}\right)$ is given by (5.33). The general form of the $\langle A A A A\rangle$ counterterm (again, neglecting a possible metric-independent part) is

$$
\begin{aligned}
\delta \widetilde{\Gamma}_{\alpha_{1} \alpha_{2} \alpha_{3} \alpha_{4}}^{\mu_{1} \mu_{2} \mu_{3} \mu_{4}}\left(l_{1}, l_{2}, l_{3}, l_{4}\right)=\left\{\eta^{\mu_{1} \mu_{2}} \eta^{\mu_{3} \mu_{4}} \delta^{b} \mathrm{q}_{\left(\alpha_{1}, \alpha_{2}\right),\left(\alpha_{3}, \alpha_{4}\right)}+\right. \\
\left.+\eta^{\mu_{1} \mu_{3}} \eta^{\mu_{2} \mu_{4}} \delta^{b} \mathrm{q}_{\left(\alpha_{1}, \alpha_{3}\right),\left(\alpha_{2}, \alpha_{4}\right)}+\eta^{\mu_{1} \mu_{4}} \eta^{\mu_{2} \mu_{3}} \delta^{b} \mathrm{q}_{\left(\alpha_{1}, \alpha_{4}\right),\left(\alpha_{2}, \alpha_{3}\right)}\right\}
\end{aligned}
$$

where the otherwise arbitrary constant tensor $\delta^{b} \mathrm{q}_{\left(\alpha_{1}, \alpha_{2}\right),\left(\alpha_{3}, \alpha_{4}\right)}$ must be symmetric w.r.t. interchanges of the grouped pairs of indices and w.r.t. interchanges of the indices within the pairs. The solution to (5.39) exists (if (4.10) is satisfied) and is unique:

$$
\begin{aligned}
-2 & (4 \pi)^{2} \delta^{b} \mathrm{q}_{\left(\alpha_{1}, \alpha_{2}\right),\left(\alpha_{3}, \alpha_{4}\right)}= \\
= & (13+8 \ln 2) \operatorname{tr}\left(\mathcal{T}_{\alpha_{1}} \mathcal{T}_{\alpha_{2}}\left\{\mathcal{T}_{\alpha_{3}}, \mathcal{T}_{\alpha_{4}}\right\}\right)-2(9+8 \ln 2) \operatorname{tr}\left(\mathcal{T}_{\alpha_{1}} \mathcal{T}_{\alpha_{3}} \mathcal{T}_{\alpha_{2}} \mathcal{T}_{\alpha_{4}}\right)+ \\
& +2(13-4 \ln 2) \operatorname{tr}\left(e_{\alpha_{1}} e_{\alpha_{2}}\left\{e_{\alpha_{3}}, e_{\alpha_{4}}\right\}\right)-4(9-4 \ln 2) \operatorname{tr}\left(e_{\alpha_{1}} e_{\alpha_{3}} e_{\alpha_{2}} e_{\alpha_{4}}\right)+ \\
& -16(1+2 \ln 2) \operatorname{tr}\left(\mathfrak{f}_{\alpha_{1}} \mathfrak{f}_{\alpha_{3}} \mathfrak{f}_{\alpha_{2}} \mathfrak{f}_{\alpha_{4}}+\mathfrak{f}_{\alpha_{1}} \mathfrak{f}_{\alpha_{4}} \mathfrak{f}_{\alpha_{2}} \mathfrak{f}_{\alpha_{3}}\right)+ \\
& +4(1+4 \ln 2) \operatorname{tr}\left(\left\{\mathfrak{f}_{\alpha_{1}}, \mathfrak{f}_{\alpha_{2}}\right\}\left\{\mathfrak{f}_{\alpha_{3}}, \mathfrak{f}_{\alpha_{4}}\right\}\right) .
\end{aligned}
$$

This completes the determination at the one-loop order of the non-minimal counterterms listed in (4.27). Adding them to the action $\tilde{I}_{0}$ obtained from $I_{0}$ according to the rules (4.11) and (4.12) one obtains the action $I_{1}$. In agreement with the results of section 4 , applying the operator $\mathcal{S}$ given by (4.2) to the effective action $\Gamma_{1}$, which is the asymptotic part (in the sense explained in section 4 ) of ${ }^{16} \Gamma\left[I_{1}^{\Lambda}\right]$ one gets in general (using (5.32) and (C.11)) that

$$
\mathcal{S}\left(\Gamma_{1}\right)=\hbar \mathcal{S}_{I_{0}} \Gamma^{(1)}+\mathcal{O}\left(\hbar^{2}\right)
$$

with (using the notation of differential forms in which $A \equiv \mathfrak{f}_{\alpha} A_{\mu}^{\alpha} \mathrm{d} x^{\mu}, \omega \equiv \mathfrak{f}_{\alpha} \omega^{\alpha}$ )

$$
\mathcal{S}_{I_{0}} \Gamma^{(1)}=-\frac{i}{24 \pi^{2}} \operatorname{tr} \int \omega \mathrm{d}\left\{A \wedge \mathrm{d} A+\frac{1}{2} A \wedge A \wedge A\right\}
$$

(in our conventions $\mathrm{d} x^{0} \wedge \mathrm{d} x^{1} \wedge \mathrm{d} x^{2} \wedge \mathrm{d} x^{3} \simeq \mathrm{d}^{4} x$ ). This means that, when minimal and non-minimal counterterms are taken into account, the Zinn-Justin identity is broken only by the true anomaly, which in our regularization and subtraction prescription (part of which is the condition that non-minimal counterterms (5.33) and (5.40) do not involve the Levi-Civita tensor) has the well known canonical form (see e.g. [21, 47]). In the rest of the paper we assume that the condition (4.10) for absence of anomalies is satisfied.

\footnotetext{
${ }^{16}$ Recall that in $I_{1}^{\Lambda}$ the substitution (3.1) is made also in the counterterms (both, minimal and nonminimal); the (momentum space) form of a regularized counterterm can be unambiguously fixed by the comparison with the corresponding regularized tree-level vertex (see the formulae (3.5)).
} 


\section{Relation between $\Lambda$ - $\overline{\mathrm{MS}}$ and DimReg- $\overline{\mathrm{MS}}$}

Having determined all one-loop counterterms, minimal ${ }^{17}$ and non-minimal ones, we can prove the equivalence at this order of the $\Lambda-\overline{\mathrm{MS}}$ scheme and the DimReg- $\overline{\mathrm{MS}}$ scheme with the naive, i.e. fully anticommuting, prescription for the $\gamma^{5}$ matrix. Equivalence at oneloop of renormalizable YM theories without scalar fields renormalized in the latter scheme and in a consistent DimReg-based scheme with the 't Hooft-Veltman-Breitenlohner-Maison prescription for $\gamma^{5}$ [32-34] has been demonstrated in [10]. Our calculation can be therefore treated as an extension of the result of [10], i.e. as a proof that at one-loop the naive DimReg- $\overline{\mathrm{MS}}$ scheme is consistent for the most general renormalizable YM theories. ${ }^{18}$ This requires relating renormalized parameters and fields in both schemes and constitutes a nontrivial check of the renormalization procedure developed in sections 4 and 5: for example, relations of the gauge couplings in the two schemes determined using different vertices must come out the same.

To make the formulae simple we denote collectively all parameters (masses and couplings) and fields (including antifields) in the $\Lambda$ - $\overline{\mathrm{MS}}$ scheme $g^{C}, C=1, \ldots$ and $\Phi$, respectively. Their counterparts in the DimReg- $\overline{\mathrm{MS}}$ scheme will be denoted $\check{g}$ and $\check{\Phi}$. Equivalence of the two schemes means that the renormalized effective action $\Gamma_{\operatorname{Dim}}[\check{\Phi}, \check{g}, \check{\mu}]$ which is the asymptotic (in the sense explained in section 4 ) part of ${ }^{19} \Gamma\left[I_{\infty}^{d}\right]$ in the naive DimReg- $\overline{\mathrm{MS}}$ can be obtained from its $\Lambda$ - $\overline{\mathrm{MS}}$ scheme counterpart $\Gamma_{\Lambda}[\Phi, g, \mu]$ - the asymptotic part of $\Gamma\left[I_{\infty}^{\Lambda}\right]$ — through a "finite renormalization" of fields and couplings:

$$
\Gamma_{\operatorname{Dim}}[\check{\Phi}, G(g), \check{\mu}]=\Gamma_{\Lambda}[\zeta \check{\Phi}, g, \check{\mu}]
$$

where $\zeta$ is a matrix field rescaling

$$
\zeta=\zeta(g)=\mathbb{1}-\frac{\hbar}{(4 \pi)^{2}} \xi_{(1)}(g)+\mathcal{O}\left(\hbar^{2}\right),
$$

and

$$
\check{g}^{C}=G^{C}(g)=g^{C}+\frac{\hbar}{(4 \pi)^{2}} \theta_{(1)}^{C}(g)+\mathcal{O}\left(\hbar^{2}\right) .
$$

The formula (6.1) assumes that the two renormalization scales: $\mu$ of the $\Lambda$ - $\overline{\mathrm{MS}}$ scheme and $\check{\mu}$ of the DimReg- $\overline{\mathrm{MS}}$ are identified (in other words, one seeks to relate fields and parameters of both schemes taken at the same numerical value of the two respective renormalization scales).

The first step in relating the two schemes is to determine the rescaling factors (matrices) $\zeta$ (6.2) for all the fields. To this end we equate the terms quadratic in the fields $\check{\Phi}$ on both sides of the condition (6.1). Having determined $\zeta$ 's in this way one can proceed to finding relations between the parameters. We consider first the matching conditions which do not depend on non-minimal counterterms.

\footnotetext{
${ }^{17}$ These can be obtained immediately from divergent parts of formulae listed in B.

${ }^{18}$ In view of this, it is natural to expect that renormalizable YM theories renormalized in the $\Lambda$ - $\overline{\mathrm{MS}}$ scheme and in DimReg-based schemes with non-naive $\gamma^{5}$ are also equivalent (at least at one-loop).

${ }^{19}$ In full analogy with the notation introduced in section $4, I_{\infty}^{d}$ denotes the dimensionally regularized action with all order counterterms included.
} 
For the scalar fields (up to the one-loop accuracy) one has the relation:

$$
\left[\zeta_{\phi}^{\mathrm{T}}\left(p^{2}-m_{S}^{2}\right) \zeta_{\phi}\right]_{i j}-\left(p^{2}-\check{m}_{S}^{2}\right)_{i j}=-\hbar \Delta_{R} \widetilde{\Gamma}_{i j}(p,-p)^{(1)}+\mathcal{O}\left(\hbar^{2}\right)
$$

On the right hand side of (6.4) the factor

$$
\Delta_{R} \widetilde{\Gamma}_{i j}(p,-p)^{(1)} \equiv \widetilde{\Gamma}_{i j}(p,-p)_{\Lambda}^{(1)}-\widetilde{\Gamma}_{i j}(p,-p)_{\operatorname{Dim}}^{(1)}
$$

is the difference of renormalized one-loop contributions in the two schemes. Since in this case the $1 \mathrm{PI}$ function $\widetilde{\Gamma}_{i j}(p,-p)_{\Lambda}^{(1)}$ (subtracted in the $\Lambda$ - $\overline{\mathrm{MS}}$ scheme) is not affected by non-minimal counterterms (cf. (4.27)), the difference (6.5) is obtained by simply setting $\delta_{\text {Div }}=\Lambda^{2}=0$ in the corresponding "bare difference" of the form (5.4) which is given explicitly by (B.11). (The formula (B.3) for $\delta_{\text {Div }}$ implies that for $\mu=\check{\mu}$ setting $\delta_{\text {Div }}=0$ is just the minimal subtraction of logarithmic divergences in both schemes). Solving (6.4) for $\zeta_{\phi}=\zeta_{\phi}^{\mathrm{T}}$ (because we work with real scalar fields) one finds (here $\hbar=1$ ):

$$
\left(\zeta_{\phi}\right)_{i j}=\delta_{i j}-\frac{1}{(4 \pi)^{2}}\left[\left\{\frac{3}{16}+\frac{1}{4} \ln 2\right\} \operatorname{tr}\left[Y_{i} Y_{j}^{*}+Y_{i}^{*} Y_{j}\right]+\left\{\frac{1}{4}+\frac{3}{8} \ln \frac{32}{9}\right\}\left(\mathcal{T}^{\alpha} \mathcal{T}_{\alpha}\right)_{i j}\right] .
$$

The formula (6.4) yields also the relation between the mass matrices $\check{m}_{S}^{2}$ and $m_{S}^{2}$ of the scalar fields in both schemes:

$$
\left[\check{m}_{S}^{2}\right]_{i j}=\left[\zeta_{\phi}^{\mathrm{T}} m_{S}^{2} \zeta_{\phi}\right]_{i j}-\Delta_{R} \widetilde{\Gamma}_{i j}(0,0)^{(1)} .
$$

We do not give the explicit form of this relation here, because it can be also obtained from the general relation between the scalar potentials in both schemes which we derive below.

In the analogous manner one finds the relation $\psi=\zeta_{\psi} \check{\psi}$ between the Majorana fields in the two schemes. Using the difference (B.13) with $\delta_{\text {Div }}$ set to zero and solving the analog of the condition (6.4) for $\zeta_{\psi}=\zeta_{F} P_{L}+\zeta_{F}^{*} P_{R}$ with Hermitian $\zeta_{F}$ one gets

$$
\zeta_{F}=\mathbb{1}+\frac{1}{(4 \pi)^{2}}\left\{\frac{1}{2} \mathfrak{f}_{\alpha} f^{\alpha}+\frac{1}{4}\left[\ln \frac{3}{4}-\frac{1}{6}\right] Y_{i}^{*} Y^{i}\right\}
$$

The mass matrices $\check{m}_{F}$ and $m_{F}$ of the left-chiral Weyl fields in the two schemes are related by

$$
\begin{aligned}
\check{m}_{F}=m_{F}+\frac{1}{(4 \pi)^{2}} & \left\{\varphi^{i} Y^{j}\left(\mathcal{T}^{\alpha} \mathcal{T}_{\alpha}\right)_{j i}-\frac{1}{2}\left[\mathfrak{f}_{\alpha}^{\mathrm{T}} \mathfrak{f}^{\alpha \mathrm{T}} m_{F}+m_{F} \mathfrak{f}_{\alpha} \mathfrak{f}^{\alpha}\right]+\right. \\
& \left.+\frac{1}{4}\left[\ln \frac{3}{4}-\frac{1}{6}\right]\left[Y^{j} Y_{j}^{*} m_{F}+m_{F} Y_{j}^{*} Y^{j}\right]\right\} .
\end{aligned}
$$

The two mass matrices depend on the background scalar fields renormalized in two different schemes: $\check{m}_{F}=\check{\mathcal{M}}_{F}(0)+\check{Y}_{i} \check{\varphi}^{i}$ and $m_{F}=\mathcal{M}_{F}(0)+Y_{i} \varphi^{i}$ (cf. (2.6)). Since in both schemes the 1PI generating functional depends only on the sum $\phi+\varphi$, it is natural to set

$$
\varphi=\zeta_{\phi} \check{\varphi}
$$


(with $\zeta_{\phi}$ given in (6.6)). This allows to rewrite (6.9) in the form (neglecting higher order terms)

$$
\begin{aligned}
\check{\mathcal{M}}_{F}(\varphi)-\mathcal{M}_{F}(\varphi)= & Y_{i}\left(\zeta_{\phi}-\mathbb{1}\right)^{i}{ }_{j} \varphi^{j}+\frac{1}{(4 \pi)^{2}}\left[\varphi^{i} Y^{j}\left(\mathcal{T}^{\alpha} \mathcal{T}_{\alpha}\right)_{j i}+\right. \\
& \left.-\frac{1}{2}\left\{\mathcal{M}_{F}(\varphi) \mathfrak{f}_{\alpha} \mathfrak{f}^{\alpha}+t p \cdot\right\}+\frac{1}{4}\left\{\ln \frac{3}{4}-\frac{1}{6}\right\}\left\{\mathcal{M}_{F}(\varphi) Y_{j}^{*} Y^{j}+t p .\right\}\right]
\end{aligned}
$$

( $t p$. stands for the transposition of the preceding term). The advantage of the relation (6.11) is that differentiating it w.r.t. $\varphi^{i}$ yields the difference of the Yukawa couplings $Y_{i}$ and $\check{Y}_{i}$ in both schemes. The result agrees with the one obtained directly from the $\phi \psi \psi$ vertex using the difference (B.15). (This confirms the relation (6.10)).

Considering the terms linear in the scalar fields $\check{\phi}$ on both sides of the condition (6.1) one gets (using (6.10)) the relation

$$
\left(\zeta_{\phi}\right)_{i}^{j} \mathcal{V}_{j}^{\prime}\left(\zeta_{\phi} \check{\varphi}\right)-\check{\mathcal{V}}_{i}^{\prime}(\check{\varphi})=\Delta_{R} \widetilde{\Gamma}_{i}(p)^{(1)}
$$

Again, $\Delta_{R} \widetilde{\Gamma}_{i}(p)^{(1)}$ is obtained from the difference (B.9) by setting in it $\delta_{\text {Div }}=\Lambda^{2}=0$. Integrating both sides of (6.12) w.r.t. the background field $\check{\varphi}$ and taking the difference of the resulting potentials $\mathcal{V}$ and $\check{\mathcal{V}}$ at the same "point" $\varphi$ one obtains the relation (neglecting higher order terms)

$$
\check{\mathcal{V}}(\varphi)-\mathcal{V}(\varphi)=-\frac{1}{2(4 \pi)^{2}} \operatorname{tr}\left\{\mathcal{M}_{V}^{2}(\varphi)^{2}\right\}+\left(\zeta_{\phi}-\mathbb{1}\right)^{i}{ }_{j} \varphi^{j} \frac{\partial}{\partial \varphi^{i}} \mathcal{V}(\varphi)
$$

Differentiating it w.r.t. the background $\varphi$ one gets the formulae relating the mass matrices and self-couplings of the scalar fields in the two schemes. The relations obtained in this way agree with the one obtained from (6.7) and the other relations obtained by considering the matching conditions relating directly the $\phi^{3}$ vertices in the two schemes.

In comparing the terms bilinear in the gauge fields on both sides of (6.1) one has to take into account also the non-minimal counterterm $\delta \widetilde{\Gamma}_{\alpha \beta}^{\mu \nu}$ (5.17) (with $\delta^{b} z_{A}$ and $\delta^{b} m_{V}^{2}$ given by (5.19) and (5.20), respectively) which affects the relevant "renormalized difference":

$$
\Delta_{R} \widetilde{\Gamma}_{\alpha \beta}^{\mu \nu}(p,-p)^{(1)}=\delta \widetilde{\Gamma}_{\alpha \beta}^{\mu \nu}(p,-p)+\left.\Delta \widetilde{\Gamma}_{\alpha \beta}^{\mu \nu}(p,-p)^{(1 B)}\right|_{\delta_{\text {Div }}=\Lambda^{2}=0},
$$

(the "bare" difference $\Delta \widetilde{\Gamma}_{\alpha \beta}^{\mu \nu}(p,-p)^{(1 B)}$ is given by (B.6)). The comparison gives

$$
\begin{aligned}
\left(\zeta_{A}\right)_{\alpha \beta}=\delta_{\alpha \beta}+\frac{1}{2(4 \pi)^{2}} & \left\{\left(\frac{11}{18}+\frac{2}{3} \ln 2\right) \operatorname{tr}\left[\mathfrak{f}_{\alpha} \mathfrak{f}_{\beta}\right]+\left(\frac{7}{144}+\frac{1}{6} \ln 2\right) \operatorname{tr}\left[\mathcal{T}_{\alpha} \mathcal{T}_{\beta}\right]+\right. \\
& \left.-\left(\frac{23}{72}+\frac{19}{6} \ln 2-\frac{3}{2} \ln 3\right) \operatorname{tr}\left[e_{\alpha} e_{\beta}\right]\right\}
\end{aligned}
$$

and

$$
\left[\check{m}_{V}^{2}\right]_{\alpha \beta}=\left[\zeta_{A}^{\mathrm{T}} m_{V}^{2} \zeta_{A}\right]_{\alpha \beta}+\Delta_{R} \widetilde{\Gamma}_{\alpha \beta}^{00}(0,0)^{(1)} .
$$


In the similar way, matching the terms proportional to the product $\phi A$ on both sides of (6.1) (using the "bare" difference (B.10), the non-minimal counterterm (5.11) and eq. (6.10)) one gets the relation

$$
\check{\mathcal{T}}_{\alpha}=\mathcal{T}_{\alpha^{\prime}}\left\{\left(\zeta_{A}\right)^{\alpha^{\prime}}+\delta \Xi^{\alpha^{\prime}}{ }_{\alpha}\right\}
$$

between the gauge group generators $\mathcal{T}_{\alpha}$ and $\check{\mathcal{T}}_{\alpha}$ in the two schemes (that is between the gauge coupling constants) in which $\delta \Xi$ is given by

$$
\delta \Xi^{\alpha^{\prime}}=\frac{1}{(4 \pi)^{2}}\left(\frac{3}{4} \ln \frac{3}{4}-\frac{1}{8}\right) \operatorname{tr}\left[e^{\alpha^{\prime}} e_{\alpha}\right],
$$

$\left(\zeta_{A}\right.$ is given in $\left.(6.15)\right)$ and the relation

$$
\check{\bar{P}}_{\alpha_{A}}=\left(\zeta_{A}\right)_{\alpha_{A}}^{\beta_{A}} \bar{P}_{\beta_{A}}
$$

between the Stueckelberg parameters (cf. (2.4)) in the two schemes.

We have verified that the formulae (6.17), (6.19) and (6.10) in conjunction with the explicit expression (2.7) for $m_{V}^{2}$ in the $\Lambda$ - $\overline{\mathrm{MS}}$ (and its DimReg counterpart) reproduce the relation (6.16). The same relation (6.17) follows also, upon using (B.12) and (5.24), from matching the $\phi \phi A$ vertices in the two schemes. Furthermore, using (6.8) together with (B.14) and (5.6) we have verified that the relation between the fermionic generators $t_{\alpha}$ and $\check{t}_{\alpha}$ in both schemes obtained by considering the vertex $\psi \psi A$ is identical to (6.17), as expected. Similarly, using eqs. (B.7) and (5.33) the same relation for the adjoint generators $e_{\alpha}$ and $\check{e}_{\alpha}$ is obtained from matching the corresponding $A A A$ vertices. Moreover, the relation (6.17) is also consistent with the form of the $A A A A$ vertices (cf. eqs. (B.16) and (5.40)).

To complete establishing the equivalence of the $\Lambda$ - $\overline{\mathrm{MS}}$ and DimReg- $\overline{\mathrm{MS}}$ schemes at the one-loop order, it is necessary to relate vertices involving antifields (these vertices do not have non-minimal counterterms). Of these only the two-point function $\left\langle K_{\alpha}^{\mu} \omega^{\gamma}\right\rangle$ has a non-vanishing "bare difference" (see B). Eq. (B.5) after minimal renormalization yields

$$
\zeta_{\mathbb{K}}^{\mathrm{T}} \zeta_{\omega}=\mathbb{1}-\frac{1}{(4 \pi)^{2}}\left(\frac{1}{8}+\frac{3}{4} \ln \frac{4}{3}\right) e_{\gamma} e^{\gamma},
$$

where $\zeta_{\mathbb{K}}$ relates the vector antifields $K^{\mu}$ and $\check{K}^{\mu}$. Introducing the notation $\Psi \equiv$ $\left(\phi, \psi, A_{\mu}, \omega\right)$ and $\mathcal{K} \equiv\left(K, \bar{K}, K^{\mu}, L\right)$ and matching the $\langle\mathcal{K} \omega \Psi\rangle$ vertices in the two schemes we get the relation

$$
\check{T}_{\gamma}^{(\Psi)}=\zeta_{\mathcal{K}}^{\mathrm{T}} T_{\gamma^{\prime}}^{(\Psi)} \zeta_{\Psi}\left(\zeta_{\omega}\right)^{\gamma^{\prime}}
$$

with $T_{\gamma}^{(\Psi)}=\left(\mathcal{T}_{\gamma}, t_{\gamma}, e_{\gamma}, e_{\gamma}\right)$. It follows that the formulae (6.20) and (6.21) are consistent with (6.17) (and its counterparts for the other kinds of generators) provided

$$
\zeta_{\mathcal{K}}=\left(\zeta_{\Psi}^{-1}\right)^{\mathrm{T}}
$$

and

$$
\left(\zeta_{\omega}\right)_{\alpha}^{\alpha^{\prime}}=\left(\zeta_{A}\right)_{\alpha}^{\alpha^{\prime}}+\delta \Xi_{\alpha}^{\alpha^{\prime}}+\mathcal{O}\left(\hbar^{2}\right)
$$


with $\delta \Xi_{\alpha}^{\alpha^{\prime}}$ introduced in (6.18). The matrix $\zeta_{\bar{\omega}}$ relating the antighost fields in both schemes is equal to $\zeta_{\mathbb{K}}$, because of the ghost equation (A.3). Similarly, the corresponding NakanishiLautrup multipliers are related by $\zeta_{h}=\left(\zeta_{A}^{\mathrm{T}}\right)^{-1}$. Finally, the block of the $\zeta_{\phi}$ matrix (6.6) corresponding to the Stueckelberg fields is the unit matrix. By comparison of the two point functions $\left\langle K_{i} \omega^{\gamma}\right\rangle$ in both schemes one concludes that the same statement holds for Stueckelberg antifields, so that (6.22) is correct in this case as well. This establishes the equivalence of the two considered schemes at least with the one-loop accuracy.

The relations between quantities defined in the $\Lambda$ - $\overline{\mathrm{MS}}$ and DimReg- $\overline{\mathrm{MS}}$ schemes found in this section, apart from providing a useful consistency test of the entire subtraction procedure defined in section 4, will allow us to obtain the two-loop RGEs satisfied by the running parameters of the former scheme using the known RGEs in the latter one. Moreover, since usually the parameters that are extracted by fitting the SM to the data are the gauge (and other) couplings in the DimReg- $\overline{\mathrm{MS}}$ scheme (at $\check{\mu}=M_{Z}$ or $M_{t}$ ), the relations established here will allow us (in section 9) to give the proper numerical input to the RGEs in the $\Lambda$ - $\overline{\mathrm{MS}}$ scheme when analyzing the hierarchy problem.

\section{Renormalization group equations}

The relations (6.3) and (6.2) imply that the one-loop RG equations in the $\Lambda$ - $\overline{\mathrm{MS}}$ and the ordinary DimReg- $\overline{\mathrm{MS}}$ schemes are identical. Moreover, having the one-loop relations between renormalized parameters and fields in the two schemes, it is possible, using the known two-loop RG equations in the DimReg- $\overline{\mathrm{MS}}$ scheme [48-51], to obtain also the two-loop RG equations for the parameters in the $\Lambda$ - $\overline{\mathrm{MS}}$ one. From the point of view of the RGE it is more convenient to treat the background $\varphi$ as a part of the scalar field $\Phi=\phi+\varphi$. The renormalized parameters of the $\Lambda$ - $\overline{\mathrm{MS}}$ scheme, collectively denoted $g^{A}$, whose two-loop $\beta$ functions are derived in this section, are therefore the gauge couplings (one per each independent gauge group factor, at least in the absence of the mixing of gauge fields corresponding to different $\mathrm{U}(1)$ groups), derivatives of the scalar potential $\mathcal{V}(\Phi)$ at $\Phi=0$, the Yukawa matrices $Y_{i}$, the mass matrices $\mathcal{M}_{F}(0)$ of the fermionic fields and the Stueckelberg parameters $\bar{P}_{\alpha_{A}}^{i}$. Before deriving these equations it is instructive, however, to take a look at how the RG arises in YM theories regularized with the help of a BRST-symmetry breaking cutoff.

The subtraction procedure defined in section 4 introduces an arbitrary mass parameter $\mu$. As a result, the action $I_{\infty}^{\Lambda}$ depends on this scale and on $\Lambda$ through the counterterms (this dependence on $\Lambda$ comes on the top on the dependence through the exponential factors (3.1)). The arbitrary scale $\mu$ is expected to play a similar role as in the DimReg- $\overline{\mathrm{MS}}$ scheme. In particular, one expects that observables computed in terms of renormalized parameters are, for fixed value of the cutoff scale $\Lambda$, independent of $\mu$, if these parameters vary appropriately with $\mu$ and that Green's functions computed in terms of renormalized parameters satisfy the appropriate differential renormalization group equations.

In the case of non-gauge theories, or if the regularization does not break the BRST invariance, the RG equations follow from the observation that $I_{\infty}^{\Lambda}$ can be written in the form of the bare action $I_{\mathrm{B}}^{\Lambda}$ which has the same form as the starting action $I_{0}^{\Lambda}$, but with the renormalized parameters $g^{A}$ replaced by the "bare" ones, $g_{\mathrm{B}}^{A}$, and with each type of field 
multiplied by its $Z^{1 / 2}$ factor. The bare parameters $g_{\mathrm{B}}^{A}$ and the $Z^{1 / 2}$ factors are constructed as power series in renormalized couplings $g^{A}$ with coefficients formally divergent in the limit of removed cutoff. The important fact (actually, more important than the precise form of $I_{\mathrm{B}}^{\Lambda}$ ) is that bare and renormalized parameters, $g_{\mathrm{B}}^{A}$ and $g^{A}$, are in the strict one-toone correspondence and that to each field corresponds a unique $Z^{1 / 2}$ factor. Thus, in this case $I_{\infty}^{\Lambda}$ depends on $\mu$ only through the $Z^{1 / 2}$ factors and the bare parameters. The formal equivalence of the perturbative expansions in renormalized parameters and in bare ones (the latter with a non-perturbative treatment of the $Z^{1 / 2}$ factors):

$$
\Gamma\left[I_{\infty}^{\Lambda}[\phi, g, \mu, \Lambda]\right]=\Gamma\left[I_{0}^{\Lambda}\left[Z^{1 / 2} \phi, g_{\mathrm{B}}\right]\right]
$$

then firstly implies, that observables computed in terms of renormalized parameters and depending explicitly on $\mu$ are in fact $\mu$-independent (if bare parameters are treated as $\mu$-independent, which is ensured by giving the renormalized parameters an implicit $\mu$ dependence, which in turn is unambiguous owing to the one-to-one correspondence of bare and renormalized parameters and uniqueness of the field renormalization $Z$ factors) and, secondly, allows, by applying to (7.1) the chain differentiation rule, to show that the effective action $\Gamma\left[I_{\infty}^{\Lambda}\right]$ satisfies the standard RG equation with beta functions which express the independence of $\mu$ (for fixed value of the UV regulator) of the bare parameters. Moreover, the equality ${ }^{20} I_{\infty}^{\Lambda}[\phi, g, \mu, \Lambda]=I_{0}^{\Lambda}\left[Z^{1 / 2} \phi, g_{\mathrm{B}}\right]$ implies also that the same RG equation is satisfied by $I_{\infty}^{\Lambda}[\phi, g, \mu, \Lambda]$.

As emphasized in [10], this standard reasoning cannot be directly extended to the BRST symmetry breaking regularizations, because the action $I_{\infty}^{\Lambda}$ constructed in the process of removing divergences and restoring the BRST invariance of the effective action does not have the form which allows for immediate identification of the $Z^{1 / 2}$ factors and bare couplings: trivially speaking, as illustrated by the explicit one-loop calculations presented in section 5, to each gauge field there correspond in fact two different $Z$ factors - one multiplying the structure $\partial_{\mu} A_{\nu} \partial^{\mu} A^{\nu}$ and another one (affected by non-minimal counterterms) multiplying $\partial_{\mu} A^{\mu} \partial_{\nu} A^{\nu}$. Furthermore, because of the non-minimal counterterms, different operator structures involving gauge fields in the interaction part of $I_{\infty}^{\Lambda}$ are multiplied by different power series (with divergent, as $\Lambda \rightarrow \infty$ and $\mu$-dependent coefficients) in renormalized couplings, so that even if it were possible to extract in each vertex the appropriate combination of field renormalization constants $Z^{1 / 2}$, one would end up with several "bare" gauge couplings $g_{\mathrm{B}}^{A(i)}$ (here $i$ labels different bare couplings corresponding to an independent gauge group factor $A$ ). It would not be then obvious that all the bare couplings $g_{\mathrm{B}}^{A(i)}$ yield the same beta function $\beta^{A} \equiv \beta^{A(i)}$ for the renormalized coupling $g^{A}$ (in other words, that requiring $\mu$ independence of one of these bare gauge couplings will automatically make $\mu$ independent also the remaining ones).

On the other hand, it is well known that the concept of bare couplings is not indispensable to prove that observables and Green's functions do satisfy the standard RG equations. Indeed, QAP allows to derive $[10,21]$ the RG equation directly in terms of the $\Gamma$ functional.

\footnotetext{
${ }^{20}$ In the regularization of section 3 the relation $I_{\infty}^{\Lambda}[\phi, g, \mu, \Lambda]=I_{0}^{\Lambda}\left[Z^{1 / 2} \phi, g_{\mathrm{B}}\right]$ is ensured (in theories without gauge symmetries) provided the substitution (3.1) is made in all counterterm vertices.
} 
However, since from our point view the action $I_{\infty}^{\Lambda}[\phi, g, \mu, \Lambda]$ should have the physical interpretation of a bare action, it is important to show that $I_{\infty}^{\Lambda}[\phi, g, \mu, \Lambda]$ and $\Gamma$ obey the same RGE. Therefore below, (modifying the reasoning of [21]) we present a recursive proof of this important fact.

We first notice that ${ }^{21} I_{0}, I_{0}^{\Lambda}$ and $\Gamma_{0}$ trivially satisfy the following relations

$$
R_{0} I_{0}=R_{0} I_{0}^{\Lambda}=R_{0} \Gamma_{0}=0, \quad \text { where } \quad R_{0} \equiv \mu \frac{\partial}{\partial \mu} .
$$

In the next step, defining the differential operator

$$
R_{n}=\mu \frac{\partial}{\partial \mu}+\beta_{n}^{C} \frac{\partial}{\partial g^{C}}-\mathcal{N}_{\phi}\left(\gamma_{n}^{\phi}\right)-\mathcal{N}_{\psi}\left(\gamma_{n}^{\psi}\right)-\mathcal{N}_{\omega}\left(\gamma_{n}^{\omega}\right)-\mathcal{N}_{A}\left(\gamma_{n}^{A}\right),
$$

in which the "counting operators" $\mathcal{N}_{X}, X=\phi, \psi, \omega, A$ are given by (4.23), while $\beta_{n}^{C}$ and $\gamma_{n}^{X}$, are some $\Lambda$-independent coefficients, we prove that if

$$
R_{n} I_{n}=\bar{r}_{n} \equiv \hbar^{n+1} r_{n}+\mathcal{O}\left(\hbar^{n+2}\right),
$$

then also

$$
R_{n} \Gamma_{n}=\hbar^{n+1} r_{n}+\mathcal{O}\left(\hbar^{n+2}\right)
$$

with precisely the same local functional $r_{n}$. The proof, relegated to $\mathrm{D}$, relies on the fact that the regularization (3.1) is such that (7.4) implies that the regularized functional $I_{n}^{\Lambda}$ automatically obeys a similar equation ${ }^{22}$

$$
R_{n} I_{n}^{\Lambda}=\bar{r}_{n}^{\Lambda}=\hbar^{n+1} r_{n}^{\Lambda}+\mathcal{O}\left(\hbar^{n+2}\right),
$$

where $\bar{r}_{n}^{\Lambda}$ is obtained from $\bar{r}_{n}$ by the replacement (3.1), so that

$$
\bar{r}_{n}^{\Lambda}=\bar{r}_{n}+\mathcal{O}\left(\Lambda^{-1}\right),
$$

because quadratically divergent terms of $I_{n}$ are momentum-independent.

To argue that (7.4) can be extended to the next order we notice that the functional

$$
J_{n+1} \equiv r_{n}+\mu \frac{\partial}{\partial \mu} \delta \Gamma_{\text {tot }}^{(n+1)}
$$

where the complete counterterm $\delta \Gamma_{\text {tot }}^{(n+1)}=-\Gamma_{n}^{(n+1) \text { div }}+\delta_{b} \Gamma_{n}^{(n+1)}$ is constructed as in section 4 , belongs to the kernel of $\mathcal{S}_{I_{0}}$. This follows from the fact that, owing to the structure of the counting operators (4.23), $R_{n}$ given by (7.3) satisfies the identity

$$
R_{n} \mathcal{S}(F)=\mathcal{S}_{F} R_{n} F
$$

\footnotetext{
${ }^{21}$ Recall (see section 4), that $\Gamma_{n}$ is obtained from the 1PI effective action $\Gamma\left[I_{n}^{\Lambda}\right]$ by neglecting terms that vanish in the infinite cutoff limit.

${ }^{22}$ Since it is $I_{n}^{\Lambda}$ that generates Feynman rules, in the reasoning of D it is crucial that $I_{n}^{\Lambda}$ (rather than $I_{n}$ ) obeys the RGE (7.6). For this it is crucial that the derivatives in counterterms have to be also replaced according to the rule (3.1); otherwise there would be no coefficients $\beta_{1}$ and $\gamma_{1}$ for which the condition $R_{1} I_{1}^{\Lambda}=\mathcal{O}\left(\hbar^{2}\right)$ would be satisfied.
} 
in which $F$ is an arbitrary functional. This allows to write $R_{n} \mathcal{S}\left(\Gamma_{n}\right)$ in two different ways:

$$
R_{n} \mathcal{S}\left(\Gamma_{n}\right)=\mathcal{S}_{\Gamma_{n}} R_{n} \Gamma_{n}=\hbar^{n+1} \mathcal{S}_{I_{0}} r_{n}+\mathcal{O}\left(\hbar^{n+2}\right),
$$

and, using (4.4),

$$
R_{n} \mathcal{S}\left(\Gamma_{n}\right)=\hbar^{n+1} R_{n} \Omega_{n}+\mathcal{O}\left(\hbar^{n+2}\right)=\hbar^{n+1} \mu \frac{\partial}{\partial \mu} \Omega_{n}+\mathcal{O}\left(\hbar^{n+2}\right) .
$$

Combining both results and recalling that $\Omega_{n}=-\mathcal{S}_{I_{0}} \delta \Gamma_{\text {tot }}^{(n+1)}$ we find that indeed $\mathcal{S}_{I_{0}} J_{n+1}=0$.

In the similar way one can show recursively that $r_{n}$ (and hence $J_{n+1}$ ) satisfies the homogeneous versions of the auxiliary conditions listed in appendix A.1. As an element of $\mathcal{V} \cap \operatorname{ker} \mathcal{S}_{I_{0}}$ (for the definition of the space $\mathcal{V}$ see the text above eq. (4.21)), $J_{n+1}$ can be represented in the form (cf. (4.22)):

$$
J_{n+1}=-\delta R I_{0} \equiv-\left\{\delta \beta^{A} \frac{\partial}{\partial g^{A}}-\mathcal{N}_{\phi}\left(\delta \gamma^{\phi}\right)-\mathcal{N}_{\psi}\left(\delta \gamma^{\psi}\right)-\mathcal{N}_{\omega}\left(\delta \gamma^{\omega}\right)-\mathcal{N}_{A}\left(\delta \gamma^{A}\right)\right\} I_{0},
$$

with some coefficients ${ }^{23} \delta \beta^{A}, \delta \gamma^{X}$. Defining then $\beta_{n+1}^{A}=\beta_{n}^{A}+\hbar^{n+1} \delta \beta^{A}$ etc. it is easy to see that $\left(R_{n+1} \equiv R_{n}+\hbar^{n+1} \delta R\right)$

$$
\begin{aligned}
R_{n+1} I_{n+1} & =R_{n} I_{n}+\hbar^{n+1} R_{n} \delta \Gamma_{\mathrm{tot}}^{(n+1)}+\hbar^{n+1} \delta R I_{0}+\mathcal{O}\left(\hbar^{n+2}\right) \\
& =\hbar^{n+1}\left\{r_{n}+\mu \frac{\partial}{\partial \mu} \delta \Gamma_{\mathrm{tot}}^{(n+1)}+\delta R I_{0}\right\}+\mathcal{O}\left(\hbar^{n+2}\right)
\end{aligned}
$$

Since $J_{n+1}=-\delta R I_{0}$, the curly bracket vanishes and we get $R_{n+1} I_{n+1}=\mathcal{O}\left(\hbar^{n+2}\right)$. The reasoning presented in $\mathrm{D}$ then shows that also $R_{n+1} \Gamma_{n+1}=\mathcal{O}\left(\hbar^{n+2}\right)$. This in turn implies that the coefficients in $\delta R$ are $\Lambda$-independent. On the other hand, the relation $\delta R I_{0}=$ $-J_{n+1}$ tells us that coefficients of $\delta R$ are polynomials in dimensional parameters of $I_{0}$; this (in conjunction with their $\Lambda$-independence) ensures that they do not depend explicitly on $\mu$. This completes the inductive step.

The above result shows that $R_{\infty} I_{\infty}=R_{\infty} I_{\infty}^{\Lambda}=0$ and, therefore (D), $R_{\infty} \Gamma_{\infty}=0$. The solution of the first of these equations by the method of characteristics tells us in general [52] that the value of $I_{\infty}$ at a "point" $(\Phi, g, \mu)$ is equal to the value assumed by $I_{\infty}$ at a particular point $\left(\Phi_{\Sigma}, g_{\Sigma}, \mu_{\Sigma}\right)$ on an arbitrarily chosen hypersurface $\Sigma$ of codimension one, connected to the point $(\Phi, g, \mu)$ by the characteristic curves specified by the equations

$$
\begin{aligned}
\frac{\mathrm{d}}{\mathrm{d} t} \bar{\mu}(t, \mu) & =\bar{\mu}(t, \mu), & \bar{\mu}(0, \mu) & =\mu, \\
\frac{\mathrm{d}}{\mathrm{d} t} \bar{g}^{A}(t, g) & =\beta^{A}(\bar{g}(t, g)), & \bar{g}^{A}(0, g) & =g^{A}, \\
\frac{\mathrm{d}}{\mathrm{d} t} \bar{\Phi}^{i}(t, \Phi, g) & =-[\gamma(\bar{g}(t, g))]_{j}^{i} \bar{\Phi}^{j}(t, \Phi, g), & \bar{\Phi}^{i}(0, \Phi, g) & =\Phi^{i} .
\end{aligned}
$$

\footnotetext{
${ }^{23}$ Note that the conditions (4.24)-(4.26) impose some constraints on these coefficients; the most interesting one of them relates the beta functions of gauge coupling to the anomalous dimension (in the Landau gauge) of the corresponding ghost field $\delta \beta^{C} \partial T_{\alpha} / \partial g^{C}=\left[\delta \gamma^{\omega}\right]_{\alpha}^{\kappa} T_{\kappa}$.
} 
In the case of the $\Lambda$ - $\overline{\mathrm{MS}}$ scheme distinguished is the hypersurface $\Sigma$ defined by the condition $(\bar{\Lambda}$ is defined in $(4.13))$

$$
f\left(\Phi_{\Sigma}, g_{\Sigma}, \mu_{\Sigma}\right) \equiv \mu_{\Sigma}-\bar{\Lambda}=0,
$$

on which $I_{\infty}$ takes the simplest form because all minimal logarithmically divergent as $\Lambda \rightarrow \infty$ counterterms vanish there (non-vanishing are only the minimal counterterms proportional to $\Lambda^{2}$ and the non-minimal ones). Thus,

$$
I_{\infty}[\Phi, g, \mu, \Lambda]=I_{\infty}\left[\Phi_{\Sigma}, g_{\Sigma}, \mu_{\Sigma}, \Lambda\right]=I_{\infty}\left[\bar{\Phi}\left(t_{\mu}^{\Lambda}, \Phi, g\right), \bar{g}\left(t_{\mu}^{\Lambda}, g\right), \bar{\Lambda}, \Lambda\right] .
$$

where (cf. eq. (4.13))

$$
t_{\mu}^{\Lambda}=\ln \frac{\bar{\Lambda}}{\mu}=\frac{1}{2} \delta_{\Lambda}
$$

The formula (7.13) together with the identification $\Phi_{\mathrm{B}} \equiv \bar{\Phi}\left(t_{\mu}^{\Lambda}, \Phi, g\right)$, provides the definition of the bare action $I_{\mathrm{B}}$ as the action defined on $\Sigma$ :

$$
I_{\mathrm{B}}\left[\Phi_{\mathrm{B}}, g_{\mathrm{B}}\right] \equiv I_{\infty}\left[\Phi_{\Sigma}, g_{\Sigma}, \mu_{\Sigma}, \Lambda\right] .
$$

The bare couplings (cubic and quartic couplings in the scalar field potential, Yukawa couplings, gauge couplings as well as the explicit mass parameters of fermions) are then naturally defined as

$$
g_{\mathrm{B}}^{A} \equiv \bar{g}^{A}\left(t_{\mu}^{\Lambda}, g\right) .
$$

Independence of $g_{\mathrm{B}}^{A}$ of $\mu$, that is $\mu(\mathrm{d} / \mathrm{d} \mu) g_{\mathrm{B}}^{A}=0$, determines then, as usual, the $\mu$ dependence of the running couplings $g^{A}(\mu)$. Since the autonomous ordinary differential equations (7.11) imply automatically that [52]

$$
\frac{\partial}{\partial t} \bar{g}^{A}(t, g)=\beta^{C}(g) \frac{\partial}{\partial g^{C}} \bar{g}^{A}(t, g),
$$

one obtains $\mu(\mathrm{d} / \mathrm{d} \mu) g^{A}(\mu)=\beta^{A}(g(\mu))$ as the RG equations allowing to relate $g^{A}(\mu)$ to $g^{A}\left(\mu^{\prime}\right)$. (Inverting the relations (7.16) expresses, of course, $g^{A}(\mu)$ through the bare couplings $g_{\mathrm{B}}^{A}$.)

According to this definition of the bare couplings, in the bare action the coefficients of the various gauge field dependent interaction vertices (affected by non-minimal counterterms) are given by different infinite power series in the bare couplings. ${ }^{24}$ Furthermore, the $Z_{A}=Z_{A}(g, \mu, \Lambda)$ factor of a gauge field $A$ is in this way uniquely defined (it is the coefficient of the $\partial_{\mu} A_{\nu} \partial^{\mu} A^{\nu}$ structure in $I_{\infty}$ which is not affected by non-minimal counterterms), whereas the coefficient of the structure $\partial_{\mu} A^{\mu} \partial_{\nu} A^{\nu}$ must be of the form $Z_{A} \times F\left(\bar{g}\left(t_{\mu}^{\Lambda}, g\right)\right) \equiv Z_{A} \times F\left(g_{\mathrm{B}}\right)$ with $F\left(g_{\mathrm{B}}\right)$ being an infinite powers series in the (dimensionless) bare couplings. Finally, the bare masses squared of the scalar fields are uniquely defined by (7.15) as the coefficients of the terms quadratic in bare scalar fields and have the form (notice that on the left hand side of (7.15) there in no explicit $\Lambda$ dependence!)

$$
\begin{aligned}
\left(m_{\mathrm{B}}^{2}\right)^{C} & =(4 \pi)^{-2} \Lambda^{2} f^{C}\left(\bar{\lambda}\left(t_{\mu}^{\Lambda}, \lambda\right)\right)+\left(\bar{m}^{2}\right)^{C}\left(t_{\mu}^{\Lambda}, m^{2}, \rho, \lambda\right) \\
& =(4 \pi)^{-2} \Lambda^{2} f^{C}\left(\lambda_{\mathrm{B}}\right)+\left(\bar{m}^{2}\right)^{C}\left(t_{\mu}^{\Lambda}, m^{2}, \rho, \lambda\right),
\end{aligned}
$$

\footnotetext{
${ }^{24}$ The exception are the terms coupling the ghost and gauge fields which, having no non-minimal counterterms, are simply proportional to the bare gauge couplings (7.16).
} 
where $\lambda$ denote generically parameters of dimension 0 (gauge, Yukawa and quartic scalar couplings) and $\rho$ stands for generic cubic scalar couplings or explicit fermionic mass parameters. It is also important to notice that because of the minimal counterterms proportional to $\Lambda^{2}$ (as well as due to the presence of non-minimal counterterms) the bare action $I_{\mathrm{B}}$ includes also bare vector boson masses squared

$$
\begin{aligned}
\left(M_{V}^{2}\right)_{\mathrm{B}} & =\Lambda^{2} H\left(\bar{\lambda}\left(t_{\mu}^{\Lambda}, \lambda\right)\right)+K\left(\bar{m}^{2}\left(t_{\mu}^{\Lambda}, m^{2}, \rho, \lambda\right), \bar{\rho}\left(t_{\mu}^{\Lambda}, \rho, \lambda\right), \bar{\lambda}\left(t_{\mu}^{\Lambda}, \lambda\right)\right) \\
& =\Lambda^{2} H\left(\lambda_{\mathrm{B}}\right)+K\left(\bar{m}^{2}\left(t_{\mu}^{\Lambda}, m^{2}, \rho, \lambda\right), \rho_{\mathrm{B}}, \lambda_{\mathrm{B}}\right),
\end{aligned}
$$

— in the cutoff regularization there are unavoidably quadratically divergent corrections also to the vector boson two-point functions (see section 5).

Summarizing, we have shown, that the action $I_{\infty}$ obtained in the process of constructing minimal and non-minimal counterterms indeed winds up to a "bare" action $I_{\mathrm{B}}$ which has no explicit dependence on $\mu$ : the entire dependence on $\mu$ enters through the bare parameters and the field renormalization factors $Z$. In particular, the result (7.18) provides the general justification of the conjecture first formulated in [53] and used in $[2,13]$, namely that coefficients of quadratic divergences are $\Lambda$-independent functions of bare couplings. It should be stressed once again, that this result relies on the consistent application of the regularization prescription of section 3 (that is, on making the substitution (3.1) also in the counterterms).

After these considerations we return to the derivation of the two-loop beta functions in the $\Lambda$ - $\overline{\mathrm{MS}}$ scheme. The relation (6.1) allows us to express the beta functions and the field anomalous dimensions in $\Lambda$ - $\overline{\mathrm{MS}}$ in terms of their DimReg- $\overline{\mathrm{MS}}$ counterparts

$$
\begin{aligned}
\beta^{A}(g) & =\left[\Omega(g)^{-1}\right]_{C}^{A} \check{\beta}^{C}(G(g)), \\
\gamma(g) & =\zeta(g) \check{\gamma}(G(g))[\zeta(g)]^{-1}+\beta^{A}(g) \zeta(g) \frac{\partial}{\partial g^{A}}[\zeta(g)]^{-1},
\end{aligned}
$$

(matrix multiplications in the second line are implicit), where $[\Omega(g)]_{A}^{C} \equiv \partial G^{C}(g) / \partial g^{A}$. Expanding ${ }^{25}$ the relations (7.20) in powers of $\hbar$ and using the differential operators (cf. eqs. (6.2)-(6.3))

we get

$$
\mathcal{B}=\check{\beta}_{(1)}^{C}(g) \frac{\partial}{\partial g^{C}}, \quad \Theta=\theta_{(1)}^{C}(g) \frac{\partial}{\partial g^{C}},
$$

$$
\begin{aligned}
\beta^{A}(g) & =\check{\beta}^{A}(g)+\frac{\hbar^{2}}{(4 \pi)^{4}}\left\{\Theta \check{\beta}_{(1)}^{A}(g)-\mathcal{B} \theta_{(1)}^{A}(g)\right\}+\mathcal{O}\left(\hbar^{3}\right), \\
\gamma(g) & =\check{\gamma}(g)+\frac{\hbar^{2}}{(4 \pi)^{4}}\left\{\Theta \check{\gamma}_{(1)}(g)+\mathcal{B} \xi_{(1)}(g)-\left[\xi_{(1)}(g), \check{\gamma}_{(1)}(g)\right]\right\}+\mathcal{O}\left(\hbar^{3}\right) .
\end{aligned}
$$

Instead of listing the beta functions for various couplings $g^{C}$ we follow Jack and Osborn [48] and give formulae for the quantities

$$
\beta_{(1)}^{T_{\alpha}} \equiv \mathcal{B} T_{\alpha}, \quad \beta_{(1)}^{\mathcal{M}_{F}}(\varphi) \equiv \mathcal{B} \mathcal{M}_{F}(\varphi), \quad \beta_{(1)}^{\mathcal{V}}(\varphi) \equiv \mathcal{B} \mathcal{V}(\varphi)
$$

\footnotetext{
${ }^{25}$ We use the obvious notation

$$
\check{\beta}(\check{g})=\sum_{\ell=1}^{\infty} \frac{\hbar^{\ell}}{(4 \pi)^{2 \ell}} \check{\beta}_{(\ell)}(\check{g}), \quad \check{\gamma}(\check{g})=\sum_{\ell=1}^{\infty} \frac{\hbar^{\ell}}{(4 \pi)^{2 \ell}} \check{\gamma}_{(\ell)}(\check{g}) .
$$
}


Since the scalar background $\varphi$ is not one of the couplings $g^{C}, \beta_{(1)}^{\mathcal{V}}(\varphi)$ is simply the scalar potential $\mathcal{V}$ present in the Lagrangian (2.1) but with each coupling replaced by its one-loop beta function. Similarly, the beta function of the Yukawa matrices can be immediately obtained as the derivatives

$$
\beta_{(1)}^{Y_{i}}=\frac{\partial}{\partial \varphi^{i}} \beta_{(1)}^{\mathcal{M}_{F}}(\varphi)
$$

The explicit forms of (7.24) $\operatorname{read}^{26}[48]$

$$
\begin{aligned}
\beta_{(1)}^{\mathcal{M}_{F}}(\varphi)= & 3\left\{\mathcal{M}_{F}(\varphi) \mathfrak{f}_{\alpha} \mathfrak{f}^{\alpha}+t p .\right\}+2 Y_{j} \mathcal{M}_{F}(\varphi)^{*} Y^{j}+ \\
& +\frac{1}{2}\left\{\mathcal{M}_{F}(\varphi) Y_{j}^{*} Y^{j}+t p \cdot\right\}+\frac{1}{2} \varphi^{i} Y^{j} \operatorname{tr}\left\{Y_{i} Y_{j}^{*}+c c .\right\}, \\
\beta_{(1)}^{\mathcal{V}}(\varphi)= & \frac{1}{2} \operatorname{tr}\left\{\mathcal{M}_{S}^{2}(\varphi)^{2}\right\}-\operatorname{tr}\left\{\left[\mathcal{M}_{F}(\varphi) \mathcal{M}_{F}(\varphi)^{*}\right]^{2}\right\}+\frac{3}{2} \operatorname{tr}\left\{\mathcal{M}_{V}^{2}(\varphi)^{2}\right\}+ \\
& +\gamma_{(1) j}^{\phi i} \varphi^{j} \frac{\partial}{\partial \varphi^{i}} \mathcal{V}(\varphi),
\end{aligned}
$$

where

$$
\gamma_{(1) i j}^{\phi}(g)=\check{\gamma}_{(1) i j}^{\phi}(g)=\frac{1}{2} \operatorname{tr}\left\{Y_{i} Y_{j}^{*}+c c .\right\}+3\left(\mathcal{T}_{\alpha} \mathcal{T}^{\alpha}\right)_{i j},
$$

is the the one-loop anomalous dimension of the scalar fields in the Landau gauge (see e.g. [49-51]). Finally, the well-known expression for the beta functions of the gauge couplings has the form

$$
\beta_{(1)}^{T_{\alpha}}=\mathcal{B} T_{\alpha}=T_{\kappa} \mathcal{A}_{\alpha}^{\kappa}, \quad T_{\alpha}=\mathfrak{f}_{\alpha}, \mathcal{T}_{\alpha}, e_{\alpha}
$$

with

$$
\mathcal{A}_{\kappa \alpha}=\frac{11}{3} \operatorname{tr}\left\{e_{\kappa} e_{\alpha}\right\}-\frac{1}{6} \operatorname{tr}\left\{\mathcal{T}_{\kappa} \mathcal{T}_{\alpha}\right\}-\frac{2}{3} \operatorname{tr}\left\{\mathfrak{f}_{\kappa} \mathfrak{f}_{\alpha}\right\} .
$$

For completeness we give here also the one-loop anomalous dimensions of the vector fields and the left-chiral Weyl fermions (in the Landau gauge)

$$
\begin{aligned}
\gamma_{(1) \kappa \alpha}^{A}(g) & =\check{\gamma}_{(1) \kappa \alpha}^{A}(g)=\frac{13}{6} \operatorname{tr}\left\{e_{\kappa} e_{\alpha}\right\}-\frac{1}{6} \operatorname{tr}\left\{\mathcal{T}_{\kappa} \mathcal{T}_{\alpha}\right\}-\frac{2}{3} \operatorname{tr}\left\{\mathfrak{f}_{\kappa} \mathfrak{f}_{\alpha}\right\} . \\
\gamma_{(1)}^{F}(g) & =\check{\gamma}_{(1)}^{F}(g)=\frac{1}{2} Y_{i}^{*} Y^{i} .
\end{aligned}
$$

In complete analogy with (7.24) we define also

$$
\theta_{(1)}^{\mathcal{M}_{F}}(\varphi) \equiv \Theta \mathcal{M}_{F}(\varphi)=(4 \pi)^{2}\left(\check{\mathcal{M}}_{F}(\varphi)-\mathcal{M}_{F}(\varphi)\right)
$$

etc.; their explicit forms follow immediately from (6.11), (6.13) and (6.17); e.g.

$$
\theta_{(1)}^{T_{\alpha}}=\Theta T_{\alpha}=(4 \pi)^{2}\left(\check{T}_{\alpha}-T_{\alpha}\right)=T_{\kappa} \Omega_{\alpha}^{\kappa},
$$

where

$$
\begin{aligned}
\Omega_{\alpha \beta}= & \left(\frac{11}{36}+\frac{1}{3} \ln 2\right) \operatorname{tr}\left\{\mathfrak{f}_{\alpha} \mathfrak{f}_{\beta}\right\}+\left(\frac{7}{288}+\frac{1}{12} \ln 2\right) \operatorname{tr}\left\{\mathcal{T}_{\alpha} \mathcal{T}_{\beta}\right\}+ \\
& -\left(\frac{41}{144}+\frac{19}{12} \ln 2-\frac{3}{4} \ln \frac{9}{4}\right) \operatorname{tr}\left\{e_{\alpha} e_{\beta}\right\}
\end{aligned}
$$

\footnotetext{
${ }^{26}$ The one-loop functions given below can also be obtained from the formulae listed in B, or, more specifically, from their parts proportional to $\delta_{\text {Div }}$.
} 
We are now in a position to compute the differences of the beta functions in the two schemes. The formula (7.22) allows us to obtain these differences by means of simple algebraic manipulations ${ }^{27}$ on objects $\beta_{(1)}^{\mathcal{X}}$ and $\theta_{(1)}^{\mathcal{X}}$. The results can be conveniently expressed in terms of the two-loop counterparts of (7.24), i.e.

$$
\begin{aligned}
& \beta_{(2)}^{\mathcal{M}_{F}(\varphi)} \equiv \beta_{(2)}^{A}(g) \frac{\partial}{\partial g^{A}} \mathcal{M}_{F}(\varphi), \\
& \check{\beta}_{(2)}^{\mathcal{M}_{F}}(\varphi) \equiv \check{\beta}_{(2)}^{A}(g) \frac{\partial}{\partial g^{A}} \mathcal{M}_{F}(\varphi),
\end{aligned}
$$

etc. In the case of the gauge couplings we get

$$
\beta_{(2)}^{T_{\alpha}}(g)=\check{\beta}_{(2)}^{T_{\alpha}}(g)+T_{\kappa}[\Omega, \mathcal{A}]_{\alpha}^{\kappa} .
$$

Both $\Omega$ and $\mathcal{A}$ are matrices of invariant forms on a Lie algebra, hence the commutator in (7.38) vanishes if the gauge group contains at most a single $\mathrm{U}(1)$ factor. In such a case the functions $\beta_{(1)}$ and $\theta_{(1)}$ for a given gauge coupling depend only on this coupling and the two-loop beta function is the same in both schemes, similarly as in theories with a single coupling. In theories with multiple U(1) factors there are more Abelian gauge couplings than independent Abelian generators and all of them can mix with each other (see e.g. [57] and references therein). The two-loop beta functions for Abelian couplings are then in general different in both schemes.

The beta functions of the couplings parametrizing the potential $\mathcal{V}$ can be obtained from

$$
\begin{aligned}
\beta_{(2)}^{\mathcal{V}}(\varphi)= & \check{\beta}_{(2)}^{\mathcal{V}}(\varphi)+\left[\gamma_{(2)}^{\phi}(g)-\check{\gamma}_{(2)}^{\phi}(g)\right]_{j}^{i} \varphi^{j} \frac{\partial \mathcal{V}(\varphi)}{\partial \varphi^{i}}+ \\
& +2 \operatorname{tr}\left\{\mathcal{M}_{V}^{2}(\varphi)^{2}[\mathcal{A}+3 \Omega]\right\}-2 \mathcal{M}_{V}^{2}(\varphi)_{\alpha \beta} \varphi^{\mathrm{T}}\left[3 \xi_{(1)}^{\phi}-\gamma_{(1)}^{\phi}\right] \mathcal{T}^{\alpha} \mathcal{T}^{\beta} \varphi+ \\
& -2 \operatorname{tr}\left\{\mathcal{M}_{S}^{2}(\varphi)^{2} \xi_{(1)}^{\phi}\right\}+2 \mathcal{M}_{V}^{2}(\varphi)_{\alpha \beta} \operatorname{tr}\left\{\mathcal{M}_{S}^{2}(\varphi) \mathcal{T}^{\alpha} \mathcal{T}^{\beta}\right\}+ \\
& -\varphi^{\mathrm{T}}\left\{\mathcal{T}^{\alpha}, \mathcal{T}^{\beta}\right\} \mathcal{M}_{S}^{2}(\varphi)\left\{\mathcal{T}_{\alpha}, \mathcal{T}_{\beta}\right\} \varphi+2 \operatorname{tr}\left\{\mathfrak{f}^{\alpha *} \mathfrak{f}_{\alpha}^{*}\left(\mathcal{M}_{F}(\varphi) \mathcal{M}_{F}(\varphi)^{*}\right)^{2}+c c .\right\}+ \\
& -2\left[\mathcal{T}^{\alpha} \mathcal{T}_{\alpha} \varphi\right]^{j} \operatorname{tr}\left\{Y_{j} \mathcal{M}_{F}(\varphi)^{*} \mathcal{M}_{F}(\varphi) \mathcal{M}_{F}(\varphi)^{*}+c c .\right\}+ \\
& -\left[\ln \frac{3}{4}-\frac{1}{6}\right] \operatorname{tr}\left\{Y^{j} Y_{j}^{*}\left(\mathcal{M}_{F}(\varphi) \mathcal{M}_{F}(\varphi)^{*}\right)^{2}+c c .\right\},
\end{aligned}
$$

where $\xi_{(1)}^{\phi}$ is the one-loop contribution to $\zeta_{\phi}$ given in (6.6) taken with the opposite sign (in agreement with the definition (6.2)). The two-loop anomalous dimension of the scalar fields in the Landau gauge reads

$$
\begin{aligned}
\gamma_{(2) i j}^{\phi}(g)= & \check{\gamma}_{(2) i j}^{\phi}(g)+\left\{\left[\frac{1}{2}+\frac{3}{4} \ln \frac{32}{9}\right] \mathcal{A}^{\kappa \lambda}+6 \Omega^{\kappa \lambda}\right\}\left(\mathcal{T}_{\kappa} \mathcal{T}_{\lambda}\right)_{i j}+ \\
& +\left[\frac{5}{4}+3 \ln 2\right] \operatorname{tr}\left\{Y_{i}^{*} Y_{j} \mathfrak{f}^{\alpha} \mathfrak{f}_{\alpha}+c c .\right\}+\left[\frac{3}{4}+\ln 2\right] \operatorname{tr}\left\{Y_{i}^{*} Y^{\ell} Y_{j}^{*} Y_{\ell}+c c .\right\}+ \\
& +\left[\frac{7}{24}+\frac{1}{2} \ln \frac{3}{2}\right] \operatorname{tr}\left\{\left(Y_{i} Y_{j}^{*}+Y_{j} Y_{i}^{*}\right) Y^{\ell} Y_{\ell}^{*}\right\}+\left[\frac{3}{4}-\frac{3}{8} \ln \frac{32}{9}\right]\left(\mathcal{T}^{\kappa} \mathcal{T}_{\kappa}\right)_{i \ell} \mathbb{Y}_{j}^{\ell},
\end{aligned}
$$

\footnotetext{
${ }^{27}$ Note that $\beta_{(1)}^{\mathcal{X}}$ and $\theta_{(1)}^{\mathcal{X}}$ are linear combinations of $\beta_{(1)}^{A}(g)$ and $\theta_{(1)}^{A}(g)$, respectively, with $g$-independent coefficients.
} 
where

$$
\mathbb{Y}_{i j} \equiv \operatorname{tr}\left\{Y_{i} Y_{j}^{*}+c c .\right\} .
$$

For the beta function of the Weyl fermions mass matrices one obtains

$$
\begin{aligned}
& \beta_{(2)}^{\mathcal{M}_{F}}(\varphi)=\check{\beta}_{(2)}^{\mathcal{M}_{F}}(\varphi)+4\left[\mathcal{T}^{\alpha} \mathcal{T}_{\alpha}-\xi_{(1)}^{\phi}\right]_{i j} Y^{i} \mathcal{M}_{F}(\varphi)^{*} Y^{j}+ \\
& +\left[\mathcal{T}^{\alpha} \mathcal{T}_{\alpha}-\xi_{(1)}^{\phi}-\frac{1}{4}\left(\ln \frac{3}{4}-\frac{1}{6}\right) \mathbb{Y}\right]_{i j}\left\{Y^{i} Y^{j *} \mathcal{M}_{F}(\varphi)+t p .\right\}+ \\
& +\varphi^{i} Y^{j}\left\{\left[\frac{3}{4}-\frac{3}{8} \ln \frac{32}{9}\right]\left[\mathbb{Y} \mathcal{T}^{\kappa} \mathcal{T}_{\kappa}-2 \mathcal{A}^{\lambda \kappa} \mathcal{T}_{\lambda} \mathcal{T}_{\kappa}\right]_{i j}+\left[\frac{5}{4}+3 \ln 2\right] \operatorname{tr}\left[Y_{i} Y_{j}^{*} \mathfrak{f}_{\alpha}^{*} \mathfrak{f}^{\alpha *}+c c .\right]\right. \\
& \left.+\left[\frac{7}{24}+\frac{1}{2} \ln \frac{3}{2}\right] \operatorname{tr}\left[Y_{i} Y_{j}^{*} Y^{\ell} Y_{\ell}^{*}+c c .\right]+\left[\frac{3}{4}+\ln 2\right] \operatorname{tr}\left[Y_{i} Y_{\ell}^{*} Y_{j} Y^{\ell *}+c c .\right]\right\} \\
& +\left[\ln \frac{3}{4}-\frac{1}{6}\right]\left\{\left(Y^{j} \mathcal{M}_{F}(\varphi)^{*} Y^{\ell} Y_{\ell}^{*} Y_{j}+t p .\right)-\left(Y^{j} Y_{\ell}^{*} Y_{j} Y^{\ell *} \mathcal{M}_{F}(\varphi)+t p .\right)\right\}+ \\
& -\left[\frac{3}{2} \ln \frac{3}{4}+\frac{1}{4}\right]\left\{\left(\mathcal{M}_{F}(\varphi) Y_{\ell}^{*} \mathfrak{f}_{\alpha}^{*} \mathfrak{f}^{\alpha *} Y^{\ell}+t p .\right)+\left(Y^{\ell} Y_{\ell}^{*} \mathfrak{f}_{\alpha}^{*} \mathfrak{f}^{\alpha *} \mathcal{M}_{F}(\varphi)+t p .\right)\right\}+ \\
& -2\left(Y^{\ell} \mathfrak{f}_{\alpha} \boldsymbol{f}^{\alpha} \mathcal{M}_{F}(\varphi)^{*} Y_{\ell}+t p .\right)+\left(\mathcal{A}^{\kappa \lambda}+6 \Omega^{\kappa \lambda}\right)\left(\mathcal{M}_{F}(\varphi) \mathfrak{f}_{\kappa} \mathfrak{f}_{\lambda}+t p .\right) .
\end{aligned}
$$

The two-loop anomalous dimension of the left-chiral Weyl fields (in the Landau gauge) reads

$$
\begin{aligned}
\gamma_{(2)}^{F}(g)= & \check{\gamma}_{(2)}^{F}(g)-\mathcal{A}^{\kappa \lambda} \mathfrak{f}_{\kappa} \mathfrak{f}_{\lambda}+\left[\mathcal{T}^{\alpha} \mathcal{T}_{\alpha}-\xi_{(1)}^{\phi}-\frac{1}{4}\left(\ln \frac{3}{4}-\frac{1}{6}\right) \mathbb{Y}\right]_{i j} Y^{i *} Y^{j}+ \\
& -\left[\frac{3}{2} \ln \frac{3}{4}+\frac{1}{4}\right]\left\{Y_{\ell}^{*} \mathfrak{f}_{\alpha}^{*} \mathfrak{f}^{\alpha *} Y^{\ell}+\mathfrak{f}^{\alpha} \mathfrak{f}_{\alpha} Y_{\ell}^{*} Y^{\ell}\right\}-\left[\ln \frac{3}{4}-\frac{1}{6}\right] Y^{\ell *} Y_{j} Y_{\ell}^{*} Y^{j},
\end{aligned}
$$

while that of the vector fields has the form

$$
\gamma_{(2) \alpha \beta}^{A}(g)=\check{\gamma}_{(2) \alpha \beta}^{A}(g)+[\Omega, \mathcal{A}]_{\alpha \beta}+\left\{\left[\frac{3}{2} \ln \frac{3}{4}-\frac{1}{4}\right] \mathcal{A}_{\alpha \gamma}-3 \Omega_{\alpha \gamma}\right\} \operatorname{tr}\left(e^{\gamma} e_{\beta}\right) .
$$

One should expect that the relation $\beta^{T_{\alpha_{A}}}=T_{\kappa_{A}}\left(\gamma^{A}\right)^{\kappa_{A}}{ }_{\alpha_{A}}$ holds in both schemes, ${ }^{28}$ so that (7.43) agrees with (7.38). Similarly, the beta functions for Stueckelberg parameters in (2.4) are determined by the anomalous dimensions of the Abelian vector fields

$$
\beta^{C} \frac{\partial}{\partial g^{C}} \bar{P}_{\alpha_{A}}=\bar{P}_{\beta_{A}}\left(\gamma^{A}\right)_{\alpha_{A}}^{\beta_{A}} .
$$

The above formulae have to be supplemented with the Jack-Osborn expressions [48] for $\check{\beta}_{(2)}$ functions in the DimReg- $\overline{\mathrm{MS}}$ scheme (to be distinguished from DimRed results, which are also given in [48]) and with the Machacek-Vaughn formulae [49-51] for $\check{\gamma}_{(2)}$ matrices. For completeness we list them (using our conventions) in E. The explicit expressions for the $\beta$ and $\theta$ functions in the $\mathrm{SM}$ are given in $\mathrm{F}$.

\section{The "bare" scalar potential}

As a further consistency check of the renormalization scheme defined in section 4 and as an example of dealing with the regularization (3.2) in higher orders we consider in

\footnotetext{
${ }^{28}$ For non-Abelian indices the relation $\beta^{T_{\alpha}}=T_{\kappa}\left(\gamma^{A}\right)^{\kappa}{ }_{\alpha}$ holds only in the background field gauge and provided $\gamma^{A}$ is the anomalous dimension of the background vector fields.
} 

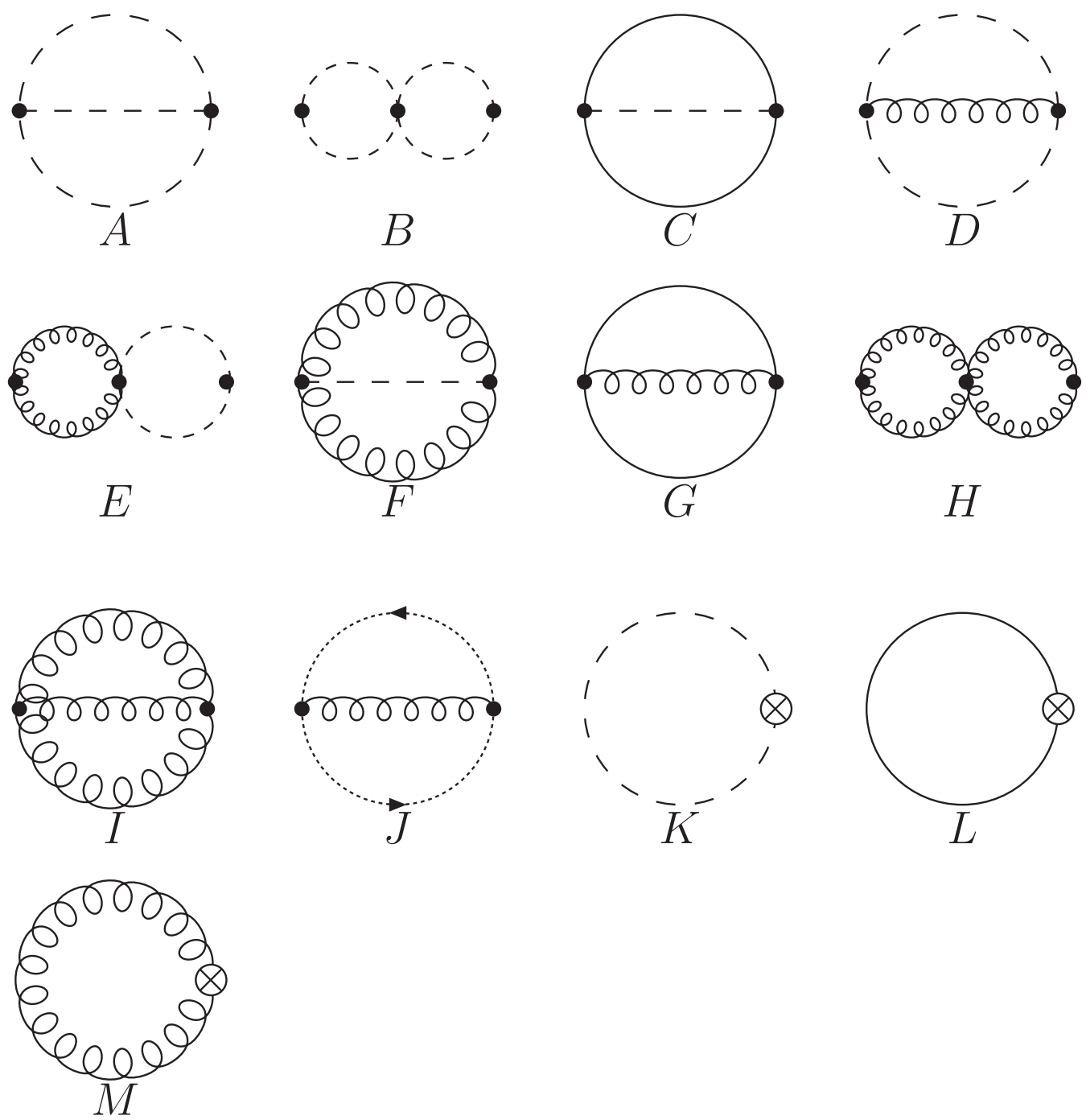

Figure 1. Order $\hbar^{2}$ vacuum graphs.

this section the order $\hbar^{2}$ contribution to the constant term $\Gamma[0]$ of the effective action $\Gamma[\phi, A, \ldots]$, i.e. to the background field dependent zero-point 1PI function. Owing to the "shift" symmetry (3.3) which is preserved by the cutoff regularization of section 3, calculating $\Gamma[0]$ in order $\hbar^{2}$ is equivalent to the determination of the two-loop contribution to the effective potential $\mathcal{V}_{\text {eff }}(\varphi)$

$$
\Gamma[\phi, \text { other (anti)fields }=0]=-\int \mathrm{d}^{4} x\left\{\mathcal{V}_{\text {eff }}(\phi+\varphi)+\text { derivative terms }\right\} .
$$

However because calculation of the complete two-loop Feynman integrals in the regularization of section 3 is quite cumbersome, here we will content ourselves ${ }^{29}$ with calculating only the divergent part of $\Gamma_{1}[0]$ (in agreement with the notation introduced in section 4 the subscript 1 indicates that the calculation proceeds from the action $\left.I_{1}^{\Lambda}\right)$. In other words, we want

\footnotetext{
${ }^{29}$ The complete two-loop $\mathcal{V}_{\text {eff }}(\varphi)$ of an arbitrary renormalizable gauge theory in the DimReg- $\overline{\mathrm{MS}}$ and DimRed schemes is given in [58].
} 
to find the $\varphi$-dependent counterterm that ensures the finiteness of $\Gamma[0]$ in the $\mathcal{O}\left(\hbar^{2}\right)$ order. This will provide some nontrivial consistency checks and will also allow to determine the two-loop coefficients of quadratic divergences in the bare action $I_{\mathrm{B}}$ introduced in section 7 .

Diagrams relevant for calculating the two-loop contribution to the zero-point function $\Gamma[0]$ are shown in figure 1 . By an appropriate change of the basis in the field space the background field dependent mass matrices $\mathcal{M}_{S}^{2}(\varphi), \mathcal{M}_{V}^{2}(\varphi)$ and $\mathcal{M}_{F}(\varphi)$ can be made diagonal. In this special basis the integrals corresponding to the genuine two-loop diagrams $A-J$ of figure 1 , which can be written down using the rules for propagators and vertices given in section 3, reduce to the nine integrals listed in G. All these integrals are fully regularized by the prescription (3.1) and can, in turn, be reduced to the four basic integrals (G.1)-(G.4) whose divergent parts we are here interested in, are determined in G. The results for divergent parts can be then written back in the initial field basis.

Diagrams $K, L$ and $M$ of figure 1 are the one-loop diagrams with insertions of the oneloop counterterms corresponding to the "wave function" and mass renormalization. We discuss them in more detail here in order to illustrate the working of our regularization scheme. As explained in section 4, the momenta in the counterterms must also be replaced according to (3.2); for example, the counterterm for the $\left\langle A_{\mu}^{\alpha} A_{\nu}^{\beta}\right\rangle_{1 \mathrm{PI}}$ function must have the form

$$
\delta_{\text {tot }} \widetilde{\Gamma}_{\alpha \beta}^{\mu \nu}(p,-p)=\eta^{\mu \nu}\left(\tilde{\delta} m_{V}^{2}\right)_{\alpha \beta}+\mathcal{R}^{\mu}(p) \mathcal{R}^{\nu}(p)\left(\delta Z_{A, L}\right)_{\alpha \beta}-\eta^{\mu \nu} \mathcal{R}(p)^{2}\left(\delta Z_{A, T}\right)_{\alpha \beta}
$$

As stressed, this is necessary for consistency of the $\Lambda$ - $\overline{\mathrm{MS}}$ scheme based on the regularization (3.2): as revealed by the analysis of section 7 (and D) only then it is possible to derive the RG equations and give the action $I_{\infty}$ the meaning of the bare action $I_{\mathrm{B}}$. Because of this rule the integrals corresponding to the diagrams $K, L$ and $M$ of figure 1 are not completely regularized by the prescription (3.1). As found in section 3, one-loop vacuum graphs are the only ones for which such a situation can occur. However, unregularized parts of these diagrams are background-independent and can be omitted in the calculation of the effective potential $\mathcal{V}_{\text {eff }}(\varphi)$. Indeed, $\mathcal{V}_{\text {eff }}(\varphi)$ can be also determined by computing the background-dependent contributions to the scalar one-point 1PI functions (i.e. to the scalar field tadpoles, which according to the analysis of section 3 get completely regularized by the prescription (3.2)), and integrating them with respect to $\varphi$. Similarly, we will omit also all other $\varphi$-independent terms proportional to $\Lambda^{4}$ in eqs. (8.17)-(8.25) below (in particular, the contribution of the ghost analog of the diagram $K$ which is background independent in the Landau gauge).

The background-dependent contributions of the diagrams $K, L, M$ reduce to the single integral

$$
\begin{aligned}
(4 \pi)^{2} \int \frac{\mathrm{d}^{4} k}{(2 \pi)^{4}} \frac{i}{\mathcal{R}(k)^{2}-m^{2}}= & \Lambda^{2}+m^{2}\left\{\ln \frac{m^{2}}{\mu^{2}}-1-\delta_{\Lambda}\right\}+ \\
& +\frac{m^{4}}{\Lambda^{2}}\left\{3 \ln \frac{m^{2}}{\mu^{2}}+\ln \frac{27}{8}-5-3 \delta_{\Lambda}\right\}+\mathcal{O}\left(\Lambda^{-3}\right)
\end{aligned}
$$


and read (tildes on $\Gamma$ indicate that the factors $(2 \pi)^{4} \delta_{\text {mom }}^{(4)}(0) \equiv \int d^{4} x$ have been removed)

$$
\begin{aligned}
\widetilde{\Gamma}(\mid K)=\frac{1}{2} \int \frac{\mathrm{d}^{4} k}{(2 \pi)^{4}} i \operatorname{tr}\left\{\left[\mathcal{R}(k)^{2}-m_{S}^{2}\right]^{-1}\left[m_{S}^{2} \delta Z_{\phi}-\tilde{\delta} m_{S}^{2}\right]\right\} \\
\widetilde{\Gamma}(\mid M)=\frac{3}{2} \int \frac{\mathrm{d}^{4} k}{(2 \pi)^{4}} i \operatorname{tr}\left\{\left[\mathcal{R}(k)^{2}-m_{V}^{2}\right]^{-1}\left[m_{V}^{2} \delta Z_{A, T}-\tilde{\delta} m_{V}^{2}\right]\right\}, \\
\widetilde{\Gamma}(\mid L)=-\int \frac{\mathrm{d}^{4} k}{(2 \pi)^{4}} i \operatorname{tr}\left\{\left[m_{F}^{*} m_{F} \delta Z_{F}+\delta Z_{F} m_{F}^{*} m_{F}-\left(m_{F}^{*} \tilde{\delta} m_{F}+\tilde{\delta} m_{F}^{*} m_{F}\right)\right] \times\right. \\
\left.\times\left[\mathcal{R}(k)^{2}-m_{F}^{*} m_{F}\right]^{-1}\right\} .
\end{aligned}
$$

(The traces reduce to simple sums over mass eigenvalues in the basis in which the mass matrices are diagonal). Here

$$
\begin{aligned}
\left(\delta Z_{A, T}\right)_{\alpha \beta} & =\frac{\delta_{\Lambda}}{(4 \pi)^{2}}\left\{-\frac{13}{6} \operatorname{tr}\left[e_{\alpha} e_{\beta}\right]+\frac{1}{6} \operatorname{tr}\left[\mathcal{T}_{\alpha} \mathcal{T}_{\beta}\right]+\frac{2}{3} \operatorname{tr}\left[\mathfrak{f}_{\alpha} \mathfrak{f}_{\beta}\right]\right\} \\
\left(\delta Z_{\phi}\right)_{i j} & =\frac{\delta_{\Lambda}}{(4 \pi)^{2}}\left\{-\frac{1}{2} \operatorname{tr}\left\{Y_{i} Y_{j}^{*}+c c .\right\}-3\left(\mathcal{T}_{\alpha} \mathcal{T}^{\alpha}\right)_{i j}\right\} \\
\delta Z_{F} & =\frac{\delta_{\Lambda}}{(4 \pi)^{2}}\left\{-\frac{1}{2} Y_{i}^{*} Y^{i}\right\}
\end{aligned}
$$

as well as (cf. eq. (2.2))

$$
\begin{aligned}
& \left(\tilde{\delta} m_{S}^{2}\right)_{i j}=\frac{\delta_{\Lambda}}{(4 \pi)^{2}}\left\{-\frac{3}{2}\left(m_{V}^{2}\right)_{\alpha \beta}\left\{\mathcal{T}^{\alpha}, \mathcal{T}^{\beta}\right\}_{i j}+\frac{3}{2}\left(\varphi^{\mathrm{T}}\left\{\mathcal{T}_{\alpha}, \mathcal{T}_{\kappa}\right\}\right)_{i}\left(\left\{\mathcal{T}^{\alpha}, \mathcal{T}^{\kappa}\right\} \varphi\right)_{j}\right. \\
& +\frac{1}{2} \lambda_{i j k l}\left(m_{S}^{2}\right)^{k l}+\frac{1}{2} \operatorname{tr}\left[\rho_{i} \rho_{j}\right]-2 \operatorname{tr}\left[Y_{j} Y_{i}^{*} m_{F} m_{F}^{*}+c c .\right]+ \\
& \left.-\operatorname{tr}\left[Y_{i} m_{F}^{*} Y_{j} m_{F}^{*}+c c .\right]\right\}+ \\
& +\frac{\Lambda^{2}}{(4 \pi)^{2}}\left\{3\left(\mathcal{T}^{\alpha} \mathcal{T}_{\alpha}\right)_{i j}-\frac{1}{2} \lambda_{i j k l} \delta^{k l}+\operatorname{tr}\left[Y_{i} Y_{j}^{*}+c c .\right]\right\} \\
& \tilde{\delta} m_{F}=\frac{\delta_{\Lambda}}{(4 \pi)^{2}}\left\{-3 \mathfrak{f}_{\gamma}^{\mathrm{T}} m_{F} \mathfrak{f}^{\gamma}+Y_{i} m_{F}^{*} Y^{i}\right\}
\end{aligned}
$$

are minimal counterterms extracted from the expressions (B.6), (B.11) and (B.13). Nonminimal counterterms enter only through the diagram $M$ in which

$$
\tilde{\delta} m_{V}^{2}=\tilde{\delta}^{0} m_{V}^{2}+\delta^{b} m_{V}^{2},
$$

consists of the minimal part

$$
\begin{aligned}
\left(\tilde{\delta}^{0} m_{V}^{2}\right)_{\alpha \beta}= & \frac{\delta_{\Lambda}}{(4 \pi)^{2}}\left\{\frac{3}{4} \operatorname{tr}\left[m_{V}^{2} e_{\alpha} e_{\beta}\right]-2 \operatorname{tr}\left[\mathfrak{f}_{\alpha} m_{F}^{*} \mathfrak{f}_{\beta}^{*} m_{F}\right]+\operatorname{tr}\left[\left\{\mathfrak{f}_{\alpha}, \mathfrak{f}_{\beta}\right\} m_{F}^{*} m_{F}\right]+\right. \\
& \left.+\frac{3}{4} \varphi^{\mathrm{T}}\left\{\mathcal{T}_{\alpha}, \mathcal{T}^{\kappa}\right\}\left\{\mathcal{T}_{\beta}, \mathcal{T}_{\kappa}\right\} \varphi\right\}+ \\
& +\frac{\Lambda^{2}}{(4 \pi)^{2}}\left\{\operatorname{tr}\left[e_{\alpha} e_{\beta}\right]+\frac{1}{2} \operatorname{tr}\left[\mathcal{T}_{\alpha} \mathcal{T}_{\beta}\right]-\operatorname{tr}\left[\mathfrak{f}_{\alpha} \mathfrak{f}_{\beta}\right]\right\}
\end{aligned}
$$


and the non-minimal one, $\delta^{b} m_{V}^{2}$, given by (5.20). The counterterm $\delta Z_{A, L}$ in (8.1) in which $\delta Z_{A, L}=\delta Z_{A, T}+\delta^{b} z_{A}$, where the non-minimal $\delta^{b} z_{A}$ part is given by (5.19), does not contribute because the vector propagator is transverse in the Landau gauge (cf. (3.4)).

In combining the contributions of the genuine two-loop diagrams $A-J$ with those of the counterterm diagrams $K, M, L$, it is convenient to decompose the diagrams $K$ - $M$ into pieces proportional to different types of couplings; schematically:

$$
L=L_{Y} \oplus L_{\mathfrak{f}}, \quad M=M_{\mathcal{T}} \oplus M_{\mathfrak{f}} \oplus M_{e} \oplus M_{\varphi}, \quad K=K_{Y} \oplus K_{\mathcal{T}} \oplus K_{\lambda} \oplus K_{\rho} \oplus K_{\varphi} .
$$

Similarly, it is convenient to decompose contributions of the fermionic two-loop diagrams $C$ and $G$ into pieces $I$ and $I I$

$$
C=C_{I} \oplus C_{I I}, \quad G=G_{I} \oplus G_{I I}
$$

corresponding to the product of, respectively, two masses and two momenta arising from numerators of propagators of the Majorana fields. As usually, combining contributions of genuine two-loop diagrams with those of the counterterm diagrams should remove all divergences non-polynomial in the background field dependent mass matrices providing thereby a nontrivial check of the consistency of the whole computation.

Having computed the divergent ( $\varphi$-dependent) contributions to the zero-point 1PI function one can determine those counterterms of $I_{2}$ which are necessary to renormalize up to the order $\hbar^{2}$ the effective potential $\mathcal{V}_{\text {eff }}$. In other words, one can determine the counterterm $\mathcal{V}_{\infty}^{(2)}(\varphi)$ in

$$
I_{2}=-\left\{\mathcal{V}(\phi+\varphi)+\sum_{\ell=1}^{2} \frac{\hbar^{\ell}}{(4 \pi)^{2 \ell}} \mathcal{V}_{\infty}^{(\ell)}(\phi+\varphi)\right\}+\ldots,
$$

(the ellipsis stand for derivative terms, and terms involving fields other than $\phi$ ). In the $\Lambda$ - $\overline{\mathrm{MS}}$ scheme defined in section 4 the functions $\mathcal{V}_{\infty}^{(\ell)}$ are pure divergences, that is, vanish if one sets first $\delta_{\Lambda}=0$ and then $\Lambda^{2}=0$. The one-loop counterterm $\mathcal{V}_{\infty}^{(1)}$ can be read off from (B.9) and reads

$$
\begin{aligned}
\mathcal{V}_{\infty}^{(1)}(\varphi)= & -\frac{\Lambda^{2}}{2}\left[\operatorname{tr}\left\{\mathcal{M}_{S}^{2}(\varphi)\right\}-2 \operatorname{tr}\left\{\mathcal{M}_{F}(\varphi) \mathcal{M}_{F}(\varphi)^{*}\right\}+3 \operatorname{tr}\left\{\mathcal{M}_{V}^{2}(\varphi)\right\}\right] \\
& +\frac{\delta_{\Lambda}}{2}\left[\frac{1}{2} \operatorname{tr}\left\{\mathcal{M}_{S}^{2}(\varphi)^{2}\right\}-\operatorname{tr}\left\{\left[\mathcal{M}_{F}(\varphi) \mathcal{M}_{F}(\varphi)^{*}\right]^{2}\right\}+\frac{3}{2} \operatorname{tr}\left\{\mathcal{M}_{V}^{2}(\varphi)^{2}\right\}\right] .
\end{aligned}
$$

We present the result for $\mathcal{V}_{\infty}^{(2)}$ dividing it (using an obvious notation, e.g. writing $\mathcal{M}_{X}$ for $\mathcal{M}_{X}(\varphi)$ and $\mathcal{M}_{X}^{4}$ for $\left[\mathcal{M}_{X}^{2}(\varphi)\right]^{2}$ etc., see also the definitions (2.2) and (7.41)) into pieces which remove divergences from the sums of genuine two-loop diagrams and the counterterm diagrams of figure 1, in which cancellations of nonlocal divergences occur:

$$
\begin{aligned}
\mathcal{V}_{\infty}^{(2)}\left(\varphi \mid A \oplus K_{\rho}\right)= & \frac{1}{4} \Lambda^{2}\left(-\delta_{\Lambda}+\ln \frac{4}{3}\right) \delta^{i j} \delta^{k m} \delta^{l n} \mathcal{V}_{i k l}^{\prime \prime \prime}(\varphi) \mathcal{V}_{j m n}^{\prime \prime \prime}(\varphi)+ \\
& +\frac{1}{8}\left(\delta_{\Lambda}^{2}-2 \delta_{\Lambda}\right) \mathcal{M}_{S}^{2}(\varphi)^{i j} \delta^{k m} \delta^{l n} \mathcal{V}_{i k l}^{\prime \prime \prime}(\varphi) \mathcal{V}_{j m n}^{\prime \prime \prime}(\varphi)
\end{aligned}
$$




$$
\begin{aligned}
& \mathcal{V}_{\infty}^{(2)}\left(\varphi \mid B \oplus K_{\lambda}\right)=-\frac{1}{4} \Lambda^{2} \delta_{\Lambda} \lambda_{i j k l} \mathcal{M}_{S}^{2 i j} \delta^{k l}+\frac{1}{8} \delta_{\Lambda}^{2} \lambda_{i j k l} \mathcal{M}_{S}^{2 i j} \mathcal{M}_{S}^{2 k l}, \\
& \mathcal{V}_{\infty}^{(2)}\left(\varphi \mid C_{I} \oplus K_{Y, I} \oplus L_{Y, I}\right)=\frac{3}{2} \Lambda^{2}\left(\delta_{\Lambda}-\ln \frac{4}{3}\right) \operatorname{tr}\left\{Y^{i} \mathcal{M}_{F}^{*} Y_{i} \mathcal{M}_{F}^{*}+c c .\right\}+ \\
& -\frac{1}{4}\left(\delta_{\Lambda}^{2}-2 \delta_{\Lambda}\right)\left\{\mathcal{M}_{S i j}^{2} \operatorname{tr}\left[Y^{i} \mathcal{M}_{F}^{*} Y^{j} \mathcal{M}_{F}^{*}+c c .\right]+2 \operatorname{tr}\left[Y^{j} \mathcal{M}_{F}^{*} Y_{j} \mathcal{M}_{F}^{*} \mathcal{M}_{F} \mathcal{M}_{F}^{*}+c c .\right]\right\}, \\
& \mathcal{V}_{\infty}^{(2)}\left(\varphi \mid C_{I I} \oplus K_{Y, I I} \oplus L_{Y, I I}\right)=\Lambda^{2} \delta_{\Lambda}\left\{\frac{3}{2} \operatorname{tr}\left[Y^{i} \mathcal{M}_{F}^{*} \mathcal{M}_{F} Y_{i}^{*}+c c .\right]-\frac{1}{4} \operatorname{tr}\left[\mathcal{M}_{S}^{2} \mathbb{Y}\right]\right\} \\
& +\Lambda^{2}\left\{\frac{1}{12}(64 \ln 2+15 \ln 3-25 \ln 5-11 \ln 11) \operatorname{tr}\left[Y^{i} \mathcal{M}_{F}^{*} \mathcal{M}_{F} Y_{i}^{*}+c c .\right]\right. \\
& \left.+\frac{1}{2}\left[-1-\frac{45}{2} \ln 3+\frac{25}{2} \ln 5+9 \ln 2\right] \operatorname{tr}\left[\mathcal{M}_{S}^{2} \mathbb{Y}\right]\right\}+ \\
& -\frac{\delta_{\Lambda}^{2}}{8}\left\{2 \operatorname{tr}\left[Y_{i}^{*}\left(\mathcal{M}_{F} \mathcal{M}_{F}^{*}\right)^{2} Y^{i}+c c .\right]+4 \mathcal{M}_{S i j}^{2} \operatorname{tr}\left[Y^{i *} \mathcal{M}_{F} \mathcal{M}_{F}^{*} Y^{j}+c c .\right]-\operatorname{tr}\left[\mathcal{M}_{S}^{4} \mathbb{Y}\right]\right\} \\
& +\delta_{\Lambda}\left\{\frac{1}{2} \operatorname{tr}\left[Y_{i}^{*} \mathcal{M}_{F} \mathcal{M}_{F}^{*} Y^{i} \mathcal{M}_{F}^{*} \mathcal{M}_{F}+c c .\right]-\frac{1}{16}(7+4 \ln 2) \operatorname{tr}\left[\mathcal{M}_{S}^{4} \mathbb{Y}\right]+\right. \\
& \left.+\frac{1}{12}\left[7+6 \ln \frac{4}{3}\right] \operatorname{tr}\left[Y_{i}^{*}\left(\mathcal{M}_{F} \mathcal{M}_{F}^{*}\right)^{2} Y^{i}+c c .\right]\right\}, \\
& \mathcal{V}_{\infty}^{(2)}\left(\varphi \mid D \oplus E \oplus K_{\mathcal{T}} \oplus M_{\mathcal{T}}\right)=\Lambda^{2} \delta_{\Lambda}\left\{-\frac{3}{2} \operatorname{tr}\left[\mathcal{M}_{S}^{2} \mathcal{T}_{\alpha} \mathcal{T}^{\alpha}\right]+\frac{7}{4} \operatorname{tr}\left[\mathcal{T}^{\alpha} \mathcal{T}^{\beta}\right] \mathcal{M}_{V \alpha \beta}^{2}\right\} \\
& +\Lambda^{2}\left\{\left[\frac{17}{8}-\frac{51}{4} \ln 2+\frac{459}{16} \ln 3-\frac{125}{8} \ln 5\right] \operatorname{tr}\left[\mathcal{T}^{\alpha} \mathcal{T}^{\beta}\right] \mathcal{M}_{V \alpha \beta}^{2}+\right. \\
& \left.+\left[\frac{29}{16}-\frac{1901}{72} \ln 2-\frac{369}{32} \ln 3+\frac{125}{72} \ln 5+\frac{847}{72} \ln 11\right] \operatorname{tr}\left[\mathcal{M}_{S}^{2} \mathcal{T}_{\alpha} \mathcal{T}^{\alpha}\right]\right\} \\
& +\frac{\delta_{\Lambda}^{2}}{8}\left\{6 \operatorname{tr}\left[\mathcal{M}_{S}^{4} \mathcal{T}_{\alpha} \mathcal{T}^{\alpha}\right]-6 \operatorname{tr}\left[\mathcal{M}_{S}^{2} \mathcal{T}^{\beta} \mathcal{T}^{\alpha}\right] \mathcal{M}_{V \alpha \beta}^{2}-\operatorname{tr}\left[\mathcal{T}^{\alpha} \mathcal{T}^{\beta}\right] \mathcal{M}_{V \alpha \beta}^{4}\right\}+ \\
& +\delta_{\Lambda}\left\{\frac{1}{96}[47+24 \ln 2] \operatorname{tr}\left[\mathcal{T}^{\alpha} \mathcal{T}^{\beta}\right] \mathcal{M}_{V \alpha \beta}^{4}-\frac{3}{8}\left[2+\ln \frac{32}{9}\right] \operatorname{tr}\left[\mathcal{M}_{S}^{4} \mathcal{T}_{\alpha} \mathcal{T}^{\alpha}\right]+\right. \\
& \left.-\frac{3}{2} \operatorname{tr}\left[\mathcal{T}_{\alpha} \mathcal{M}_{S}^{2} \mathcal{T}^{\alpha} \mathcal{M}_{S}^{2}\right]-\frac{3}{2} \operatorname{tr}\left[\mathcal{M}_{S}^{2} \mathcal{T}^{\beta} \mathcal{T}^{\alpha}\right] \mathcal{M}_{V \alpha \beta}^{2}\right\}, \\
& \mathcal{V}_{\infty}^{(2)}\left(\varphi \mid F \oplus K_{\varphi} \oplus M_{\varphi}\right)=\left[-\frac{15}{8} \Lambda^{2} \delta_{\Lambda}+\Lambda^{2}\left(-\frac{1}{8}+\frac{189}{32} \ln 2-\frac{45}{16} \ln 3\right)\right] \times \\
& \times \varphi^{\mathrm{T}}\left\{\mathcal{T}_{\alpha}, \mathcal{T}_{\beta}\right\}\left\{\mathcal{T}^{\alpha}, \mathcal{T}^{\beta}\right\} \varphi+ \\
& +\delta_{\Lambda}^{2}\left[\frac{3}{8} \varphi^{\mathrm{T}}\left\{\mathcal{T}_{\alpha}, \mathcal{T}_{\beta}\right\} \mathcal{M}_{S}^{2}\left\{\mathcal{T}^{\alpha}, \mathcal{T}^{\beta}\right\} \varphi+\frac{9}{16} \varphi^{\mathrm{T}}\left\{\mathcal{T}_{\alpha}, \mathcal{T}^{\gamma}\right\}\left\{\mathcal{T}^{\alpha}, \mathcal{T}^{\delta}\right\} \varphi \mathcal{M}_{V \gamma \delta}^{2}\right] \\
& -\delta_{\Lambda}\left[\frac{3}{8} \varphi^{\mathrm{T}}\left\{\mathcal{T}_{\alpha}, \mathcal{T}_{\beta}\right\} \mathcal{M}_{S}^{2}\left\{\mathcal{T}^{\alpha}, \mathcal{T}^{\beta}\right\} \varphi+\right. \\
& \left.+\frac{3}{32}\left(14+3 \ln \frac{32}{9}\right) \varphi^{\mathrm{T}}\left\{\mathcal{T}_{\alpha}, \mathcal{T}^{\gamma}\right\}\left\{\mathcal{T}^{\alpha}, \mathcal{T}^{\delta}\right\} \varphi \mathcal{M}_{V \gamma \delta}^{2}\right], \\
& \mathcal{V}_{\infty}^{(2)}\left(\varphi \mid G_{I I} \oplus M_{\mathfrak{f}, I I}\right)=\Lambda^{2} \delta_{\Lambda}\left\{\operatorname{tr}\left[\mathfrak{f}^{\alpha} \mathfrak{f}^{\beta}\right] \mathcal{M}_{V \alpha \beta}^{2}-\frac{3}{2} \operatorname{tr}\left[\mathcal{M}_{F}^{*} \mathcal{M}_{F} \mathfrak{f}_{\alpha} \mathfrak{f}^{\alpha}+c c .\right]\right\}+ \\
& +\Lambda^{2}\left\{\left[-\frac{13}{4}+\frac{61}{2} \ln 2-54 \ln 3+25 \ln 5\right] \operatorname{tr}\left[\mathfrak{f}^{\alpha} \mathfrak{f}^{\beta}\right] \mathcal{M}_{V \alpha \beta}^{2}+\right.
\end{aligned}
$$




$$
\begin{aligned}
&+ {\left.\left[\frac{667}{18} \ln 2+21 \ln 3+\frac{25}{9} \ln 5-\frac{187}{9} \ln 11-\frac{13}{8}\right] \operatorname{tr}\left[\mathcal{M}_{F}^{*} \mathcal{M}_{F} \mathfrak{f}_{\alpha} \mathfrak{f}^{\alpha}+c c .\right]\right\} } \\
&+ \delta_{\Lambda}^{2}\left\{\frac{3}{4} \operatorname{tr}\left[\mathcal{M}_{F}^{*} \mathcal{M}_{F} \mathfrak{f}^{\alpha} \mathfrak{f}^{\beta}+c c .\right] \mathcal{M}_{V \alpha \beta}^{2}-\frac{1}{2} \operatorname{tr}\left[\mathfrak{f}^{\alpha} \mathfrak{f}^{\beta}\right] \mathcal{M}_{V \alpha \beta}^{4}\right\}+ \\
&+ \delta_{\Lambda}\left\{-\operatorname{tr}\left[\left(\mathcal{M}_{F}^{*} \mathcal{M}_{F}\right)^{2} \mathfrak{f}^{\alpha} \mathfrak{f}_{\alpha}+c c .\right]+\frac{3}{8}(1-4 \ln 2) \operatorname{tr}\left[\mathcal{M}_{F}^{*} \mathcal{M}_{F} \mathfrak{f}^{\alpha} \mathfrak{f}^{\beta}+c c .\right] \mathcal{M}_{V \alpha \beta}^{2}\right. \\
&\left.+\frac{1}{12}(19+6 \ln 4) \operatorname{tr}\left[\mathfrak{f}^{\alpha} \mathfrak{f}^{\beta}\right] \mathcal{M}_{V \alpha \beta}^{4}\right\} \\
& \mathcal{V}_{\infty}^{(2)}\left(\varphi \mid G_{I} \oplus M_{\mathfrak{f}, I} \oplus L_{\mathfrak{f}}\right)=\Lambda^{2}\left[\frac{9}{2} \delta_{\Lambda}+\frac{9}{2} \ln 3-\frac{21}{2} \ln 2-\frac{9}{8}\right] \operatorname{tr}\left[\mathcal{M}_{F} \mathfrak{f}^{\alpha} \mathcal{M}_{F}^{*} \mathfrak{f}_{\alpha}^{*}+c c .\right] \\
&-\delta_{\Lambda}^{2}\left\{\frac{3}{2} \operatorname{tr}\left[\mathcal{M}_{F} \mathcal{M}_{F}^{*} \mathcal{M}_{F} \mathfrak{f}^{\alpha} \mathcal{M}_{F}^{*} \mathfrak{f}_{\alpha}^{*}+c c \cdot\right]+\frac{3}{4} \operatorname{tr}\left[\mathcal{M}_{F} \mathfrak{f}^{\alpha} \mathcal{M}_{F}^{*} \mathfrak{f}^{\beta *}+c c .\right] \mathcal{M}_{V \alpha \beta}^{2}\right\}+ \\
&+\delta_{\Lambda}\left\{3 \operatorname{tr}\left[\mathcal{M}_{F} \mathcal{M}_{F}^{*} \mathcal{M}_{F} \mathfrak{f}^{\alpha} \mathcal{M}_{F}^{*} \mathfrak{f}_{\alpha}^{*}+c c .\right]+\right. \\
&\left.+\frac{3}{8}(7+4 \ln 2) \operatorname{tr}\left[\mathcal{M}_{F} \mathfrak{f}^{\alpha} \mathcal{M}_{F}^{*} \mathfrak{f}^{\beta *}+c c .\right] \mathcal{M}_{V \alpha \beta}^{2}\right\} \\
& \mathcal{V}_{\infty}^{(2)}\left(\varphi \mid H \oplus I \oplus J \oplus M_{e}\right)=\Lambda^{2} \delta_{\Lambda}\left\{-\frac{35}{8} \operatorname{tr}\left[e^{\beta} e^{\alpha}\right] \mathcal{M}_{V \beta \alpha}^{2}\right\}+ \\
&+\Lambda^{2}\left[\frac{39}{4}-\frac{9695}{96} \ln 2+\frac{81}{4} \ln 3-\frac{625}{24} \ln 5+\frac{847}{24} \ln 11\right] \operatorname{tr}\left[e^{\beta} e^{\alpha}\right] \mathcal{M}_{V}^{2} \beta \alpha \\
&+\delta_{\Lambda}^{2}\left\{\frac{13}{8} \operatorname{tr}\left[e^{\beta} e^{\alpha}\right] \mathcal{M}_{V \beta \alpha}^{4}+\frac{9}{16} \operatorname{tr}\left[e_{\alpha} \mathcal{M}_{V}^{2} e^{\alpha} \mathcal{M}_{V}^{2}\right]\right\}+ \\
&-\delta_{\Lambda}\left\{\left[\frac{61}{48}+\frac{103}{16} \ln 2-\frac{27}{8} \ln 3\right] \operatorname{tr}\left[e^{\beta} e^{\alpha}\right] \mathcal{M}_{V \beta \alpha}^{4}+\right. \\
&\left.+\left[\frac{129}{16}+\frac{3}{32} \ln \frac{2^{15}}{3^{6}}\right] \operatorname{tr}\left[e_{\alpha} \mathcal{M}_{V}^{2} e^{\alpha} \mathcal{M}_{V}^{2}\right]\right\}
\end{aligned}
$$

The function $\mathcal{V}_{\infty}^{(2)}$ given by the sum of the expressions (8.17)-(8.25) is indeed polynomial in the $\varphi$-dependent masses, in agreement with the expectations. Furthermore, it has been established in section 7 that the (local) action (8.15) with the two-loop counterterms included should satisfy the RGE of the form $R_{2} I_{2}=\mathcal{O}\left(\hbar^{3}\right)$ (cf. eq. (7.3)); this, in particular, implies the following relation

$$
\beta_{(2)}^{\mathcal{V}}(\varphi)-\left[\gamma_{(2)}^{\phi}\right]_{j}^{i} \varphi^{j} \frac{\partial}{\partial \varphi^{i}} \mathcal{V}(\varphi)=v^{(2)}(\varphi)
$$

where $v^{(2)}(\varphi)$ is the coefficient of $\frac{1}{2} \delta_{\Lambda}$ (i.e. of $\ln (\Lambda / \mu)$ ) in $\mathcal{V}_{\infty}^{(2)}(\varphi)$. We have verified that $v^{(2)}(\varphi)$ extracted from the formulae (8.17)-(8.25) agrees with the left hand side of (8.26) computed using the result (7.39) combined with the DimReg result (E.2). (Notice that (7.39) gives precisely the difference appearing on the left hand side of (8.26).) Moreover, the RGE $R_{2} I_{2}=\mathcal{O}\left(\hbar^{3}\right)$ implies that the coefficients of the $\Lambda^{2} \times \delta_{\Lambda}$ and $\delta_{\Lambda}^{2}$ terms in (8.17)-(8.25) should be entirely fixed by the 1-loop divergences (8.16) and the 1-loop $\beta$ and $\gamma$ functions; we have verified that this is indeed the case. In particular, up to the (background) field renormalization, in $\mathcal{V}_{\infty}^{(2)}(\varphi)$ the terms proportional to $\Lambda^{2} \times \delta_{\Lambda}$ can be ob- 
tained from quadratic divergences in (8.16) by replacing there the renormalized couplings with the bare ones.

Finally, in the results (8.17)-(8.25) there is a new information, which is not a mere consistency check of our earlier results: this is the 2-loop coefficients of the quadratic divergence (of $\Lambda^{2}$ ) which is important for the hierarchy problem (section 9). The explicit form of this coefficient for the SM is given by (F.8). It differs from the one derived in [13] where superficially a similar regularization was used.

A possible explanation of the discrepancy of our result (F.8) and that of [13] follows from the observation that the latter one is reproduced if: i) after the reduction to the basic integrals (G.1)-(G.4) only the "sunset" integrals (G.1) contribute to the two-loop coefficients of the quadratic divergences (in other words, contributions of the remaining basic integrals are assumed to cancel exactly with the contributions of the counterterm diagrams $K, L$ and $M$, ii) all the sunset integrals (G.1) occur in the same version $n_{1}=n_{2}=n_{3}=1$. These assumptions are satisfied in the DimRed scheme because the coefficient of the quadratic divergence of the sunset integral (G.1) is in DimRed given by the residue of the pole at $d=3[11,12]$, while the quadratic divergences of the remaining $\mathcal{O}\left(\hbar^{2}\right)$ contributions correspond to residues of the poles at $d=2$. The fact that the result of [13] agrees with the one of [11, 12] suggests that the cutoff on the integrals was in [13] imposed after their reduction to the basic integrals. In contrast, our result (F.8) is obtained using the consistent implementation of the cutoff procedure of section 3 (which requires making the substitution (3.1) at the level of the complete action, including the counterterms, that is before reducing Feynman integrals to the basic ones, so that no operations on divergent integrals are performed) which violates the above assumptions (for example ii) is violated by the fermionic loop of the diagram $C$ ). It is this consistent implementation which allows to prove the RGE and is therefore the one in which the conjecture of $[13,53]$ (proved in section 7 ) is valid.

\section{Bare parameters and the hierarchy problem}

In its most applications the role of QFT is to establish relations between various low energy data. In this context renormalization allows to parametrize predictions of a concrete model, like the SM, in terms of a small set of finite parameters. Regularization is then only an auxiliary procedure which is chosen following the requirements of calculational convenience and counterterms implementing subtractions are not treated as carrying any physical information - they become infinite when, at the end, the regularization is removed. In such applications of QFT the origin and magnitude of finite parameters, like masses of physical particles, are not an issue.

With an explicit UV cutoff, like the one introduced in section 3, one can, however, take another point of view (ubiquitous in applications of field theory to critical phenomena) and, keeping the UV cutoff finite and fixed, treat the action $I_{\infty}^{\Lambda}$ with the counterterms constructed in the process of renormalization as the fundamental object - the "bare" action expressed in terms of "bare" parameters and "bare" fields. That such a bare action 
can be defined when the cutoff breaks the BRST symmetry ${ }^{30}$ has been shown in section 7 . Once such a bare action $I_{\mathrm{B}}^{\Lambda}$ is obtained, there is in fact no need to split bare parameters (and fields) into renormalized ones and counterterms: it is perfectly possible to compute Green's functions directly in terms of bare parameters (keeping the regularizing cutoff finite) - when they are used to express, order by order in the perturbative expansion, physical quantities in terms of a selected set of other physical quantities (like $M_{Z}, G_{F}^{-1 / 2}, \alpha_{\mathrm{EM}}$, etc. in the $\mathrm{SM}$ ) all potential infinities disappear leaving relations which would remain finite in the limit of removed UV cutoff (in practical application it is then convenient to remove the cutoff entirely to simplify the results; nothing however prevents in principle keeping the regulator finite, at least when no gauge fields are present - see the remarks at the end of section 4). In such an approach the UV cutoff can be given a physical meaning e.g. of the inverse of the lattice spacing of a statistical model underlying the considered field theory model or, as we want to treat it here, the characteristic scale of a more fundamental finite theory. The question why the measured masses of physical particles described by the model, like $W^{ \pm}, Z^{0}$ or the Higgs boson are orders of magnitude smaller than the value of the physical UV cutoff $\Lambda$, which should be comparable to the Planck scale, ${ }^{31}$ becomes then important and is known as the hierarchy problem.

To study the hierarchy problem as described above one has to assume that at the most fundamental level physics of all interactions, including the gravitational ones, is described by some (most probably finite) theory, which may be not a QFT, and (like Loop Quantum Gravity) may even give a completely different view on space and time, which predicts all measured quantities in terms of a single dimensionful parameter, to be identified with $\Lambda$, which is its intrinsic scale. It is then quite natural to expect that all predictions of this hypothetical fundamental theory pertaining to low energy physics (low with respect to $\Lambda$ ), in the limit in which departures of the space-time from the flat Minkowski space-time are neglected and coupling to the gravitational sector ignored, can be obtained from an effective finite field theory whose bare action $I_{\mathrm{B}}^{\Lambda}$ and bare parameters are fixed by the fundamental theory. Moreover, taking into account the putative finiteness of the fundamental theory, it is natural to assume that it is the intrinsic scale of the latter that acts in the effective theory as the UV cutoff. It is also conceivable that the complete effective field theory action $I_{\mathrm{B}}^{\Lambda}$ contains also terms suppressed by $\Lambda$ whose effect is such that amplitudes computed in the effective theory eventually do satisfy for finite $\Lambda$ all the necessary ST identities, even though $I_{\mathrm{B}}^{\Lambda}$ is not BRST invariant. In such a scenario the underlying hypothetical fundamental theory of all interactions must by itself solve the fundamental aspect of the hierarchy problem, that is predict the ratio $M_{W} / M_{\mathrm{Pl}} \sim M_{W} / \Lambda$. But even if it does, the hierarchy problem generically manifests itself at the level of the effective low energy field theory as the fine cancellation between the bare mass square parameters $m_{\mathrm{B}}^{2}$ (like (7.18))

\footnotetext{
${ }^{30}$ If there is a physical regulator preserving all symmetries necessary for quantum consistency (or as in the $\phi^{4}$ model, there is simply no continuous symmetries) there is no need to construct counterterms: it is possible to start directly from the bare action which takes then the same form as $I_{0}^{\Lambda}$.

${ }^{31}$ Each physical intermediate scale between the electroweak scale and the Planck one potentially generates a hierarchy problem, if the effective quantum field theory valid below the intermediate scale involves scalar fields; in our considerations we assume absence of such intermediate scales.
} 
of the scalar fields (if such fields are present in the low energy theory) and, as is clear from (7.19), also of bare masses squared of the vector fields $\left(M_{V}^{2}\right)_{\mathrm{B}}$ (if the built-in cutoff violates explicitly the gauge symmetry) and the order $\Lambda^{2}$ contributions in the perturbative calculation of the physical $W^{ \pm}, Z^{0}$ and the Higgs boson masses.

Of course, if it is assumed, as it must, that the fundamental theory predicts correctly the ratio $M_{W} / M_{\mathrm{Pl}}$ (and $M_{h} / M_{\mathrm{Pl}}$ ), the above cancellation is an artifact of using the effective field theory. Nevertheless it is precisely this cancellation (which can be termed the "technical" aspect of the hierarchy problem), which from the point of view of the low energy effective theory is perceived as the main hierarchy problem and attempts at solving it entirely within the effective theory, undertaken over years, have led to many ideas such as technicolor or low energy supersymmetry, extra dimensions, etc.

If one adopts this attitude toward the hierarchy problem, it is just the cutoff dependent bare action of the effective theory which is of special interest. Of course the fundamental theory is unknown and, therefore, neither the corresponding bare action nor the way the intrinsic scale $\Lambda$ of the fundamental theory acts in it as a cutoff are known. Nevertheless, it may by enlightening, using the bare action $I_{\mathrm{B}}^{\Lambda}$ of section 7 (which, with the cutoff $\Lambda$ implemented as in section 3, has many features expected from the realistic effective theory - after expanding the regularizing exponential functions (3.1) it consists of infinite set of operators of growing dimensions, coefficients of operators containing gauge fields are given by infinite series in bare couplings) and assuming a concrete form of the action $I_{0}$ - be it the SM or some of its extensions - to pursue a kind of a "bottom-up" approach and investigate the resulting structure of the bare effective action (as a function of the unknown scale $\Lambda$ ) implied by the low energy data. In particular it can be interesting within such an approach to see, using the RG equations of section 7 to evolve the renormalized parameters from the electroweak scale up to the high scale (of order $M_{\mathrm{Pl}}$ ) where they become bare parameters of $I_{\mathrm{B}}^{\Lambda}$ (see (7.16)), whether one can get some clues to the technical aspect of the hierarchy problem, at least as far as the cancellation between bare masses squared of scalar fields and the $\Lambda^{2}$ contributions are concerned. ${ }^{32}$

In [2], inspired by the study [13], we have envisaged a possibility which, if realized in Nature, would in fact imply absence of such a cancellation. This possibility - viewed from the perspective of the bottom-up approach — is the potential existence of a particular cutoff scale ${ }^{33} \bar{\Lambda}=\bar{\Lambda}_{\star}$ at which all contributions proportional to $\Lambda^{2}$ to the counterterms to the scalar field masses squared, that is all coefficients $f^{C}\left(\lambda_{\mathrm{B}}(\bar{\Lambda})\right)$ in (7.18), simultaneously vanish. If such a value of $\bar{\Lambda}$ exists and is reasonably close to Planck scale, one can take the position that $\Lambda_{\star} \approx 3 \bar{\Lambda}_{\star}$ is perhaps the intrinsic scale of the fundamental theory and that the obtained bare action $I_{\mathrm{B}}^{\Lambda}$ defined as in (7.15) is the bare action of the corresponding effective field theory. Absence of terms proportional to $\Lambda^{2}$ in (7.18), i.e. the vanishing of all coefficients $f^{C}$, would of course mean absence of the technical hierarchy problem in the effective theory. While this bears some resemblance to the well known Veltman condition [56],

\footnotetext{
${ }^{32}$ It seems, however, that the problem of a similar cancellation for vector fields must be taken care of by some other mechanism operating at the level of the fundamental theory, possibly related to the one which is necessary to restore the BRST invariance for finite $\Lambda$ - see the remarks at the end of section 4 .

${ }^{33}$ It is more convenient to work with the rescaled cutoff $\bar{\Lambda} \approx 0.32 \Lambda$ introduced in eq. (4.13).
} 
the important differences should be noted: the Veltman condition was imposed on the renormalized couplings at the electroweak scale. Moreover, if the DimRed is used as the regularization (as advocated by Veltman), the leading quadratic divergences correspond, at $L$-th loop, to simple poles at $d=4-2 / L$; therefore vanishing of quadratic divergences requires in DimRed an infinite number of constraints on coupling constants (which can be simultaneously satisfied only if there is a special symmetry, like e.g. the supersymmetry). In contrasts, in the consistent regularization based on a physical UV momentum cutoff $\Lambda$, like the one of section 3 , coefficients of quadratic divergences arising from consecutive loops combine (as shown in section 7) to cutoff independent functions of bare couplings, and the number of constraints coincides with the (finite and small) number of scalar field multiplets and, therefore, their vanishing does not require any additional (super)symmetry.

If all coefficients $f^{C}\left(\lambda_{\mathrm{B}}\right)$ in (7.18) do vanish simultaneously, the smallness of the electroweak scale $G_{F}^{-1 / 2}$ and Higgs boson mass(es) compared to the Planck scale must be ensured by the smallness compared to the scale $\Lambda$ of the functions $\left(\bar{m}^{2}\right)^{C}$ in (7.18); this, in turn, must be ensured by the fundamental theory, much in the same way as the smallness of soft supersymmetry breaking scalar masses in supersymmetric low energy effective theories must be ensured by a supersymmetry breaking mechanism operating in the underlying more fundamental theory.

In [2] using the one-loop RG equations (which are identical in $\Lambda$ - $\overline{\mathrm{MS}}$ and in DimReg$\overline{\mathrm{MS}}$ schemes) and one-loop approximation to the functions $f^{C}$ in (7.18) we have shown that the scenario described above can be realized in the extension of the SM considered earlier in $[59,60]$ and consisting of an extra complex singlet scalar field and three right-chiral gauge singlet neutrino fields. Here, using the two-loop RG equations derived in section 7 and the two-loop approximation to the functions $f^{C}$ we analyze this possibility taking for $I_{0}$ the $\mathrm{SM}$ action. One of the reasons for doing this exercise is to get an estimate on the simplest possible example of changes brought in by the systematic inclusion of all two-loop effects.

In the $\mathrm{SM}$ there is only one $\mathrm{SU}(2)$ doublet of scalar fields and, consequently, only one function $f$ defined by (7.18) (in the model analyzed in [2] there were two such functions). The one-loop contribution $f^{(1)}$ to

$$
f=f^{(1)}+(4 \pi)^{-2} f^{(2)}+\ldots
$$

can be read off from (8.16) and reads (for the normalization of the couplings - see eqs. (F.1)-(F.3); all Yukawa couplings other than the top one, $y_{t}$, are neglected):

$$
f^{(1)} \equiv-6 \lambda_{1}-\frac{3}{4}\left(3 g_{w}^{2}+g_{y}^{2}\right)+6 y_{t}^{2} .
$$

$f^{(2)}$ is given in (F.8). All couplings in (9.2) and (F.8) are the bare couplings (the subscripts B are omitted for simplicity).

To find the dependence of the SM function $f$ on the rescaled cutoff scale $\bar{\Lambda} \approx 0.32 \Lambda$ (cf. (4.13)) we evolve the SM couplings in the $\Lambda$ - $\overline{\mathrm{MS}}$ scheme using the two-loop RG equations of $\mathrm{F}$ from the scale $\mu=M_{t}$ up to some high scale. (Recall that, in agreement with (7.16), the cutoff-dependent dimensionless bare coupling $g_{\mathrm{B}}(\bar{\Lambda})$ is simply given by the running one $g(\mu)$ extrapolated to high scales, i.e. $g_{\mathrm{B}}(\bar{\Lambda})=g(\mu=\bar{\Lambda})$.) As the initial 
conditions for the RG evolution we take the known values of the SM DimReg- $\overline{\mathrm{MS}}$ scheme couplings [61]

$$
\begin{aligned}
\check{\lambda}\left(M_{t}\right)= & 0.12604+0.00206\left\{\frac{M_{h}}{\mathrm{GeV}}-125.15\right\}+ \\
& -0.00004\left\{\frac{M_{t}}{\mathrm{GeV}}-173.34\right\} \pm 0.0003_{\mathrm{th}}, \\
\check{y}_{t}\left(M_{t}\right)= & 0.93690+0.00556\left\{\frac{M_{t}}{\mathrm{GeV}}-173.34\right\} \pm 0.0005_{\mathrm{th}}, \\
\check{g}_{w}\left(M_{t}\right)= & 0.64779+0.00004\left\{\frac{M_{t}}{\mathrm{GeV}}-173.34\right\}, \\
\check{g}_{y}\left(M_{t}\right)= & 0.35830+0.00011\left\{\frac{M_{t}}{\mathrm{GeV}}-173.34\right\}, \\
\check{g}_{s}\left(M_{t}\right)= & 1.1666-0.00046\left\{\frac{M_{t}}{\mathrm{GeV}}-173.34\right\},
\end{aligned}
$$

(the central value of $g_{s}$ corresponds to $\left.\alpha_{s}\left(M_{Z}\right)=0.1184\right)$ in which $M_{t}=(173.34 \pm 0.75) \mathrm{GeV}$ and $M_{h}=(125.15 \pm 0.24) \mathrm{GeV}$ are the pole top quark and Higgs boson masses, and convert them with the help of the relation (6.3) which takes here the form

$$
g^{C}\left(M_{t}\right)=\check{g}^{C}\left(M_{t}\right)-\frac{1}{(4 \pi)^{2}} \theta_{(1)}^{C}\left(\check{g}\left(M_{t}\right)\right)
$$

with the one-loop $\theta$ functions given in (F.18)-(F.23), into the values appropriate for the $\Lambda$ - $\overline{\mathrm{MS}}$ scheme. ${ }^{34}$

The dependence of the SM function $f$ of (7.18) on the rescaled cutoff $\bar{\Lambda}$ for the central values of the couplings (9.3) is shown in figure 2 . It is seen that the two-loop effects lower the scale $\bar{\Lambda}$ at which $f$ vanishes by about 3 orders of magnitude. Nevertheless, this scale remains too high to reasonably identify $\Lambda \approx 3 \bar{\Lambda}$ with the intrinsic scale of a fundamental theory which, as argued, should be related to the Planck scale $M_{\mathrm{Pl}}=1.8 \times 10^{18} \mathrm{GeV}$.

In figure 3 we compare the results of various approaches. It is clear that replacing only $f^{(2)}$ given in [13] by the result (F.8) of the systematic calculation in the consistent regularization scheme of section 3 is not very significant numerically. The difference is larger if the actual approach taken in [13] (dashed line) is compared with our result (solid line). Still, this comparison shows that the estimate of the scale $\bar{\Lambda}$ at which $f$ vanishes is not very sensitive to the details (nor to the consistency) of the approach taken to estimate the twoloop effects. This is important for the interpretation of the hierarchy problem proposed in this section. Since the one-loop beta functions are (for mass independent schemes) universal and the function $f^{(1)}$ in (9.1) is (up to a multiplicative constant) independent of the precise form of the momentum cutoff ${ }^{35}$ it should be possible, unless large values of some couplings

\footnotetext{
${ }^{34} \mathrm{Up}$ to the two-loop accuracy we could alternatively evolve the couplings (9.3) using the two-loop RG equations of the DimReg- $\overline{\mathrm{MS}}$ scheme and convert them at the scale $\mu=\bar{\Lambda}$ into the $\Lambda$ - $\overline{\mathrm{MS}}$ scheme couplings using (9.4) with $M_{t}$ replaced by $\bar{\Lambda}$.

${ }^{35}$ At least if the cutoff does not differentiate between fields of different spins — but this seems a reasonable assumption in view of the universality of gravity.
} 


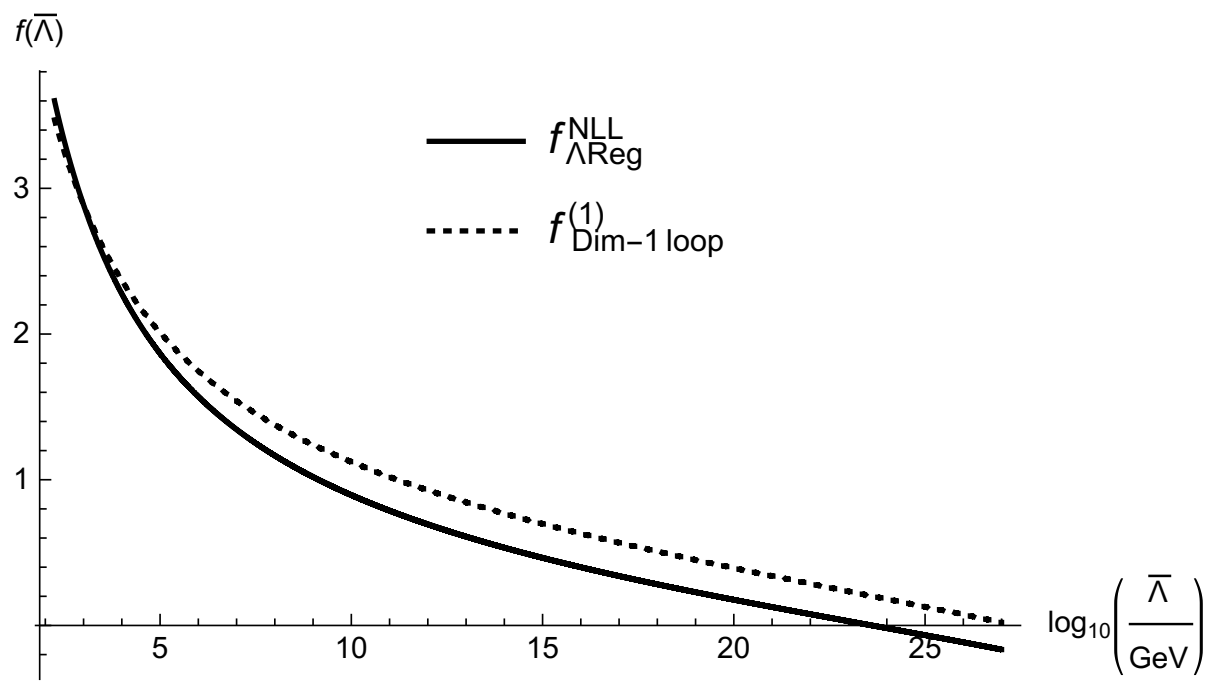

Figure 2. Coefficient $f$ of the quadratic divergence (of the term proportional to $\Lambda^{2}$ in (7.18)) in the SM. The solid line shows the results of the full two-loop (NLL) calculation (i.e. the full two-loop coefficient $f$ with $\Lambda$ - $\overline{\mathrm{MS}}$ couplings running according to two-loop beta functions). The short-dashed line shows the one-loop coefficient $f^{(1)}$ with DimReg- $\overline{\mathrm{MS}}$ couplings running according to one-loop beta functions. Both curves correspond to the central values of the DimReg- $\overline{\mathrm{MS}}$ initial data given by (9.3).

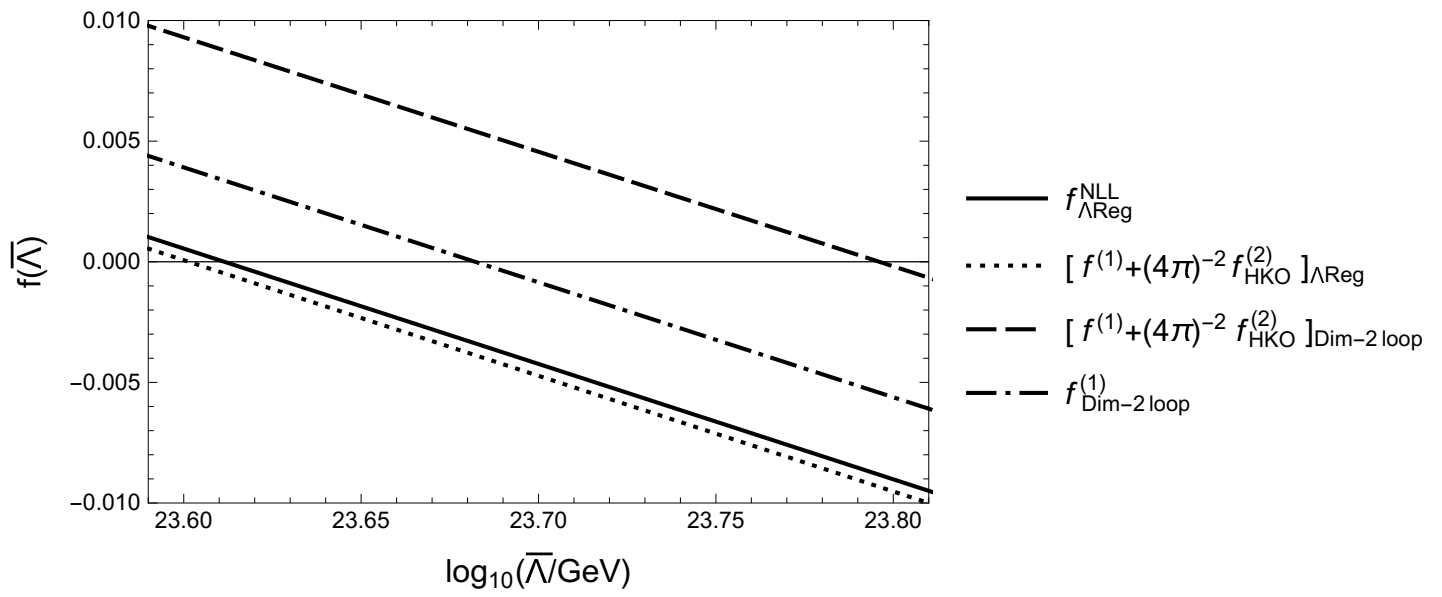

Figure 3. Comparison of the result of the consistent two-loop calculation of $f$ (solid line) with other approaches: as indicated, the dotted line shows the result of replacing $f^{(2)}$ given in (F.8) by $f^{(2)}$ of [13], the dashed line corresponds to using in addition the two-loop running couplings of the DimReg- $\overline{\mathrm{MS}}$ (instead of $\Lambda$ - $\overline{\mathrm{MS}}$ ) scheme. Finally, the dot-dashed line shows the result of approximating $f$ by $f^{(1)}$ and using the two-loop running couplings of the DimReg- $\overline{M S}$ scheme. In all cases central values of the initial values of (9.3) are used. 


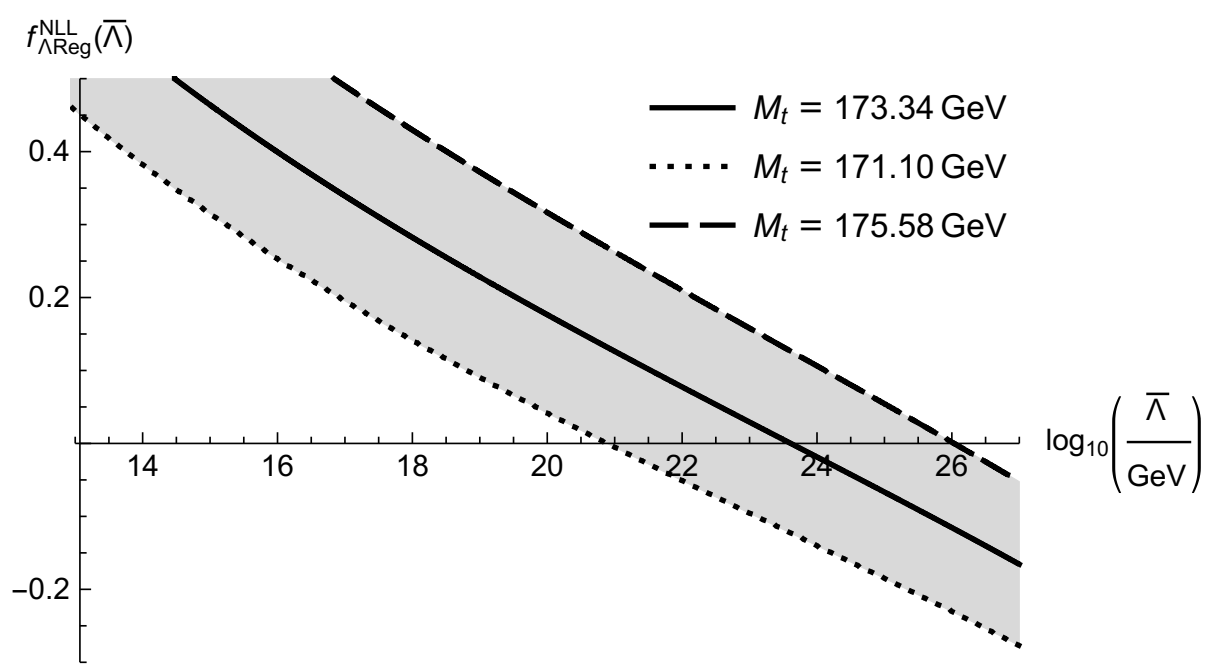

Figure 4. Uncertainty of the SM function $f_{\Lambda \text { Reg }}^{\mathrm{NLL}}$ corresponding to the uncertainty in the value of the top mass. The band corresponds to $3 \sigma$ deviations of $M_{t}$ from the central value $M_{t}=$ $(173.34 \pm 0.75) \mathrm{GeV}$. Central value of $M_{h}$ is used.

come into play, and if the uncertainty in the top mass is reduced - see below - to reliably test whether a given extension of the SM involving elementary scalar fields is consistent with the proposed solution to the hierarchy problem, that is whether it predicts (with the uncertainty of one-two orders of magnitude) $\bar{\Lambda}$ sufficiently close to the Planck scale.

As illustrated in figure 4 the value of scale $\bar{\Lambda}$ at which the SM function $f$ vanishes strongly depends on the actual value of top mass. This is, however, not surprising since the instability scale of the SM is also strongly dependent on the value of $M_{t}$ [61].

\section{Conclusions}

In this paper we have considered renormalization of a general renormalizable YM theory with scalar and spinor fields in the regularization based on a physical UV momentum cutoff which explicitly breaks the BRST symmetry. In this connection we have recalled the general renormalization procedure based on QAP. We have proposed a concrete consistent realization of such a regularization and formulated a mass-independent renormalization procedure in terms of counterterms to the action which implement the necessary subtractions. Using our scheme we have performed a systematic one-loop renormalization of a general YM theory obtaining explicitly the one-loop counterterms (minimal and non-minimal ones). The proposed renormalization scheme, similarly to the conventional $\overline{\mathrm{MS}}$ scheme, introduces an arbitrary renormalization scale $\mu$. Therefore, we have proved that the parameters and Green's functions computed in this scheme satisfy the appropriate RG equations ensuring independence of $\mu$ of physical quantities. We have also established the relations between parameters of the theory renormalized in our scheme and those of the ordinary $\overline{\mathrm{MS}}$ scheme. This allowed us to obtain explicit expressions for the two-loop RG equations satisfied by the parameters renormalized in our scheme. Their correctness has been partly checked by the direct calculation in our scheme of divergences of two-loop vacuum graphs. 
The established RG invariance of physics allowed to define the $\mu$ independent bare couplings and formulate the theory in terms of the bare action dependent on the cutoff scale $\Lambda$ only through the regularizing exponential function. The structure of this bare action has been elucidated.

Finally, the concept of the bare action allowed us to speculate in the last part of the paper on the hierarchy problem. We have formulated a condition which, if realized in Nature, could be considered a solution of this problem, at least as far as it concerns scalar fields only. It should be stressed that this solution does not require any additional (super)symmetry. Using the results of the paper we have analyzed whether the SM itself can be consistent with this possibility. While it turns out that the renormalization scale at which the parameters of the SM satisfy the necessary condition is too high to be accepted, from comparing on the example of the SM different approximations we have gained some useful insight into the reliability of the similar checks based on simple one-loop calculations for potential extensions of the SM.

\section{Acknowledgments}

We thank Hermann Nicolai for discussions. K.A.M. thanks the Albert Einstein Institute in Potsdam for hospitality and support. A.L. and K.A.M. were supported by the Polish NCN grant DEC-2013/11/B/ST2/04046.

\section{A Auxiliary conditions in the subtraction procedure}

\section{A.1 Auxiliary conditions}

Here we list the auxiliary conditions which together with the ZJ identity $\mathcal{S}(\Gamma)=0$ specify the 1PI effective action $\Gamma$. For convenience we write these conditions for an arbitrary functional $G$. These are (see [21] and references therein):

- The "translational Ward identity" [16]

$$
\tau_{i} G=0, \quad \tau_{i} \equiv-\frac{\partial}{\partial \varphi^{i}}+\int \mathrm{d}^{4} x \frac{\delta}{\delta \phi^{i}(x)} .
$$

- Symmetry w.r.t. global gauge transformations (cf. formulae (A.11)-(A.12) below for the definition of $\mathcal{W}_{\alpha}$ )

$$
\mathcal{W}_{\alpha} G=0
$$

- The ghost equation

$$
\mathcal{G}^{\alpha}(x) G=0, \quad \mathcal{G}^{\alpha}(x) \equiv \frac{\delta}{\delta \bar{\omega}_{\alpha}(x)}-\frac{\partial}{\partial x_{\mu}} \frac{\delta}{\delta K_{\alpha}^{\mu}(x)},
$$

- The (Landau) gauge condition

$$
\frac{\delta G}{\delta h_{\beta}(x)}=\bar{\Delta}_{h}^{\beta}(x), \quad \bar{\Delta}_{h}^{\beta}(x) \equiv-\partial^{\nu} A_{\nu}^{\beta}(x),
$$


- The antighost equation [62]

$$
\overline{\mathcal{G}}_{\alpha} G=\Delta_{\alpha}^{\overline{\mathcal{G}}}, \quad \overline{\mathcal{G}}_{\alpha} \equiv \int \mathrm{d}^{4} x\left\{\frac{\delta}{\delta \omega^{\alpha}(x)}-\bar{\omega}_{\gamma}(x) e_{\alpha \beta}^{\gamma} \frac{\delta}{\delta h_{\beta}(x)}\right\},
$$

in which

$$
\Delta_{\alpha}^{\overline{\mathcal{G}}} \equiv \int \mathrm{d}^{4} x\left\{L_{\beta} e_{\alpha \gamma}^{\beta} \omega^{\gamma}-K_{\beta}^{\mu} e_{\alpha \gamma}^{\beta} A_{\mu}^{\gamma}-K_{i}\left[\mathcal{T}_{\alpha}(\phi+\varphi)+\bar{P}_{\alpha}\right]^{i}+\bar{K}_{a}\left[t_{\alpha} \psi\right]^{a}\right\}
$$

If the gauge Lie algebra has an Abelian ideal, an additional condition, the local Abelian antighost equation (AAE) [45]

$$
\frac{\delta G}{\delta \omega^{\alpha_{A}}(x)}=\bar{\Delta}_{\alpha_{A}}^{\overline{\mathcal{G}}}(x)
$$

with

$$
\bar{\Delta}_{\alpha_{A}}^{\overline{\mathcal{G}}} \equiv-\partial_{\mu}\left\{K_{\alpha_{A}}^{\mu}-\partial^{\mu} \bar{\omega}_{\alpha_{A}}\right\}-K_{i}\left[\mathcal{T}_{\alpha_{A}}(\phi+\varphi)+\bar{P}_{\alpha_{A}}\right]^{i}+\bar{K}_{a}\left[t_{\alpha_{A}} \psi\right]^{a}
$$

is imposed.

If Stueckelberg fields are present in the model (i.e. at least one vector $\bar{P}_{\beta_{A}}$ in (2.4) is nonzero) one can ensure (by performing, if necessary, an orthogonal rotation in the space of scalars $\phi^{i}$ ) that only the last $N_{S t}$ rows $\bar{p}^{s}{ }_{\alpha_{A}}$ with $s=1, \ldots, N_{S t}$ of the matrix $\left[\bar{P}^{i}{ }_{\alpha_{A}}\right]$ are non-vanishing and that they are linearly independent. The corresponding components of the (rotated) scalar field $\phi$ are the Stueckelberg fields $\xi^{s}$. In such a case two further conditions (the Stueckelberg equations)

$$
\frac{\delta G}{\delta \xi^{s^{\prime}}(x)}=\bar{\Delta}_{s^{\prime}}^{\Xi}(x), \quad \bar{\Delta}_{s^{\prime}}^{\Xi} \equiv-\delta_{s^{\prime} s} \partial^{\mu}\left\{\partial_{\mu} \xi^{s}+\bar{p}_{\gamma_{A}}^{s} A_{\mu}^{\gamma_{A}}\right\}
$$

and

$$
\mathscr{T}_{\mu}^{s}(x) G=0, \quad \mathscr{T}_{\mu}^{s}(x) \equiv \frac{\partial}{\partial x^{\mu}} \frac{\delta}{\delta K_{s}(x)}+\bar{p}_{\gamma_{A}} \frac{\delta}{\delta K_{\gamma_{A}}^{\mu}(x)},
$$

are imposed. Further conditions on $I_{0}$ and on $\Gamma$ may result from imposing other continuous or discrete global (non-gauge) symmetries.

All the conditions (A.1)-(A.8) are satisfied by the tree-level action $I_{0}(2.9)$ in the Landau gauge (2.11) expressing their "accidental symmetries" or specifying their breaking (factors $\Delta$ ). It the analysis it is important that because all the $\Delta$ factors are linear in the quantum (propagating) fields they do not affect quantum corrections.

Most of the conditions (A.1)-(A.8) play only a simplifying role in our analysis: imposed on $\Gamma$, they enforce the Landau gauge as a particular choice in the class of $R_{\xi}$ gauges. An important exception is the Abelian antighost equation (A.6) which specifies Abelian gauge currents beyond the tree-level: if the theory has continuous (non-gauge) symmetries, in $\operatorname{ker} \mathcal{S}_{I_{0}}$ there are terms corresponding to couplings of Abelian gauge fields to conserved currents of these symmetries [25, 44]. Such terms, which unlike other elements of ker $\mathcal{S}_{I_{0}}$, do not correspond to infinitesimal changes of parametrization of the tree-level action $I_{0}$, 
are excluded by the AAE $[25,45]$. That this is indeed so can be seen by noticing that for an arbitrary functional $F[45]$ the following "anti-commutation relation" holds:

$$
\frac{\delta}{\delta \omega^{\alpha_{A}}(x)} \mathcal{S}(F)+\mathcal{S}_{F}\left(\frac{\delta F}{\delta \omega^{\alpha_{A}}(x)}-\bar{\Delta}_{\alpha_{A}}^{\overline{\mathcal{G}}}(x)\right)=\mathfrak{W}_{\alpha_{A}}(x) F-\partial_{\mu} \partial^{\mu} h_{\alpha_{A}}(x)
$$

Here $\mathfrak{W}_{\alpha_{A}}(x)$ is the infinitesimal generator of Abelian gauge transformations

$$
\begin{aligned}
\mathfrak{W}_{\alpha_{A}}= & \partial_{\mu} \frac{\delta}{\delta A_{\mu}^{\alpha_{A}}}+\left[\mathcal{T}_{\alpha_{A}}(\phi+\varphi)+\bar{P}_{\alpha_{A}}\right]^{i} \frac{\delta}{\delta \phi^{i}}+\left[t_{\alpha_{A}} \psi\right]^{a} \frac{\delta}{\delta \psi^{a}}+ \\
& -K_{j}\left[\mathcal{T}_{\alpha_{A}}\right]_{i}^{j} \frac{\delta}{\delta K_{i}}-\bar{K}_{b}\left[t_{\alpha_{A}}\right]_{a}^{b} \frac{\delta}{\delta \bar{K}_{a}}
\end{aligned}
$$

From (A.9) one learns that if $\Gamma$ satisfies the ZJ identity and the AAE then it also obeys Abelian Ward-Takahashi (WT) identities which ensure that Abelian gauge bosons couple only to gauge currents.

(Anti)commutation relations, similar to (A.9), hold also for all other differential operators in (A.1)-(A.8) (some of them can be found in [21]). Here we show only the one satisfied by $\overline{\mathcal{G}}_{\alpha}$ in order to specify the $\mathcal{W}_{\alpha}$ operator in (A.2)

$$
\overline{\mathcal{G}}_{\alpha} \mathcal{S}(F)+\mathcal{S}_{F}\left(\overline{\mathcal{G}}_{\alpha} F-\Delta_{\alpha}^{\overline{\mathcal{G}}}\right)=\mathcal{W}_{\alpha} F
$$

In particular, comparing (A.11) with (A.9) we get the relation between $\mathcal{W}_{\alpha_{A}}$ and (A.10)

$$
\mathcal{W}_{\alpha_{A}}=\int \mathrm{d}^{4} x \mathfrak{W}_{\alpha_{A}}(x)
$$

Relations like (A.11) mean that for functionals $G$ which satisfy the ZJ identity (4.1) not all conditions (A.1)-(A.8) are independent. They are, however, all necessary to specify the actions $I_{n}$ which do not satisfy this identity.

Finally, we remark that, as can be seen from (4.2), gauge singlet fields are in our formalism treated on an equal footing with non-singlet ones. In particular, we do not exclude the possibility that antifields corresponding to gauge singlets (i.e. $L_{\alpha_{A}}$ and, say, $K_{i_{s}}$ and $\bar{K}_{a_{s}}$ ) appear in counterterms even though they are absent in the tree-level action $I_{0}$. Assigning to them the same ghost numbers and power-counting dimensions as to their non-singlet counterparts one concludes that the conditions

$$
\frac{\delta I_{n}}{\delta K_{\alpha_{A}}^{\mu}}=-\partial_{\mu} \omega^{\alpha_{A}}, \quad \frac{\delta I_{n}}{\delta L_{\alpha_{A}}}=0, \quad \frac{\delta I_{n}}{\delta K_{i_{s}}}=0, \quad \frac{\delta I_{n}}{\delta \bar{K}_{a_{s}}}=0,
$$

follow already from the conditions (A.1)-(A.8) imposed on the $I_{n}$ functional - they do not have to be imposed separately. ${ }^{36}$

\footnotetext{
${ }^{36}$ Alternatively, these new constraints can be imposed by appropriately restricting the form of the $\mathcal{S}(\cdot)$ operation $[24,25]$.
} 


\section{A.2 Completion of the inductive step}

To complete the inductive step discussed in section 4 we have to show that the auxiliary conditions (A.1)-(A.8) are satisfied by $I_{n+1}$. To this end, we first notice that the identities (A.1), (A.2) and (A.5) are preserved by the regularization prescription (3.1), i.e. if $I_{n}$ obeys them, then so does $I_{n}^{\Lambda}$. By using the well-known arguments [22] one concludes that $\Gamma_{n}^{\Lambda}$ and its "asymptotic part" $\Gamma_{n}$ satisfy these identities. In particular, this means that $\Gamma_{n}^{(n+1) \text { div }}$ obeys their homogeneous counterparts. The same arguments show that $\Gamma_{n}^{(n+1) d i v}$ possesses all global (non-gauge) symmetries of $I_{0}$. In fact, $\Gamma_{n}^{(n+1) \text { div }}$ satisfies also homogeneous versions of all the remaining conditions listed in appendix A.1, even though, due to their dependence on derivatives, these identities are not (exactly) preserved by the regularization (3.1). Let us consider first (A.3) with $G=I_{n}$. This identity implies that $I_{n}$ depends on the antighost $\bar{\omega}_{\alpha}$ only through the difference $K_{\alpha}^{\mu}-\partial^{\mu} \bar{\omega}_{\alpha}$. That the same is true also for $\Gamma_{n}$ is obvious from Feynman diagrams, the only subtlety being that the derivative acting on the antighost field in $I_{n}^{\Lambda}$ is replaced according to (3.1). However, the additional exponential that could spoil this identity for $\Gamma_{n}^{\Lambda}$ necessarily contains an external momentum. Thus, the breaking of identity (A.3) for $\Gamma_{n}^{\Lambda}$ tends to zero in the infinite cutoff limit (1PI functions with external antighost lines are at most linearly divergent, due to the derivative on each antighost field in the action $I_{n}$ ). Thus, the entire $\Gamma_{n}^{(n+1)}$ and in particular, $\Gamma_{n}^{(n+1) \text { div }}$ satisfy the identity (A.3). The same arguments show that $\Gamma_{n}^{(n+1) \text { div }}$ obeys (A.8). Finally, the conditions, (A.4), (A.6) and (A.7) applied to $I_{n}$ restrict its vertices in such a way that it is impossible to construct 1PI loop diagrams which would contribute to functions with external lines of, respectively, $h_{\alpha}, \omega^{\alpha_{A}}$ and $\xi^{\epsilon_{G}}$. Thus, $\Gamma_{n}^{(n+1) \operatorname{div}}$ obeys homogeneous versions of these identities as well.

To prove that $I_{n+1}$ obeys (A.1)-(A.8) we still have to show that the non-minimal counterterm $\delta_{b} \Gamma_{n}^{(n+1)}$ in eq. (4.18) satisfies their homogeneous versions. This is an important point since a priori these identities could be in conflict with the condition (4.17) of restoration of the ZJ identity. That this is not the case follows from (anti)commutation relations like (A.11). More precisely, the above arguments show that the $\tilde{I}_{n}$ functional, cf. (4.11), obeys all the conditions (A.1)-(A.8). Similarly as above one concludes that $\tilde{\Gamma}_{n}$ obeys them as well. Thus, the relation (A.11) applied to $F=\tilde{\Gamma}_{n}$ in conjunction with (4.15) tells us that $\tilde{\Omega}_{n}$ obeys the homogeneous version of the antighost equation (A.5)

$$
\overline{\mathcal{G}}_{\alpha} \tilde{\Omega}_{n}=0 .
$$

Using the counterparts of (A.11) for the other functional differential operators appearing in (A.1)-(A.8) one concludes that $\tilde{\Omega}_{n}$ satisfies also homogeneous versions of all the remaining conditions (A.1)-(A.8) except for the Abelian antighost equation (A.6). ${ }^{37}$ Ex-

\footnotetext{
${ }^{37}$ The difference between the Abelian antighost equation and other auxiliary identities is caused by the fact that the Abelian WT identity is badly broken by our regularization prescription and thus $\tilde{\Gamma}_{n}$ does not satisfy it. From (A.9) we get

$$
\hbar^{n+1} \frac{\delta \tilde{\Omega}_{n}}{\delta \omega^{\alpha_{A}}(x)}+\mathcal{O}\left(\hbar^{n+2}\right)=\mathfrak{W}_{\alpha_{A}}(x) \tilde{\Gamma}_{n}-\partial_{\mu} \partial^{\mu} h_{\alpha_{A}}(x)
$$

with $\mathfrak{W}_{\alpha_{A}}(x)$ defined in (A.10). Incidentally this relation shows that the counterterms which remove the breakings $\tilde{\Omega}_{n}$ (4.15) automatically restore also Abelian WT identities.
} 


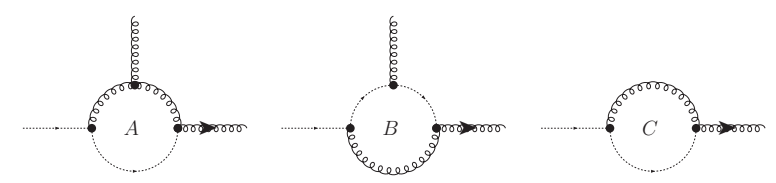

Figure 5. Corrections to the BRST transformation of vector fields.

ploiting these constraints and assuming that $\tilde{\Omega}_{n}$ is cohomologically trivial (cf. the remarks below eq. (4.9)),

$$
\tilde{\Omega}_{n}=\mathcal{S}_{I_{0}} \tilde{\mathcal{C}}_{n}
$$

we have verified that $\tilde{\mathcal{C}}_{n}$ must be the sum of two terms

$$
\tilde{\mathcal{C}}_{n}=\tilde{\mathcal{C}}_{n}^{0}+\tilde{\mathcal{C}}_{n}^{1}
$$

of which $\tilde{\mathcal{C}}_{n}^{0}$ satisfies the homogeneous versions of all the conditions (A.1)-(A.8) (including (A.6)) and can be assumed to be invariant under global and discrete symmetries of $I_{0}$, while $\tilde{\mathcal{C}}_{n}^{1}$ belongs to $\operatorname{ker} \mathcal{S}_{I_{0}}$. Let us consider $\left(\right.$ A.1) as an example. For $\tilde{\mathcal{C}}_{n}=\tilde{\mathcal{C}}_{n}[\phi, \ldots ; \varphi]$ one can define $\tilde{\mathcal{C}}_{n}^{0}$ as

$$
\tilde{\mathcal{C}}_{n}^{0}=\tilde{\mathcal{C}}_{n}^{0}[\phi, \ldots ; \varphi] \equiv \tilde{\mathcal{C}}_{n}[\phi+\varphi, \ldots ; 0]
$$

so that

$$
\begin{aligned}
\tilde{\mathcal{C}}_{n}^{1}=\tilde{\mathcal{C}}_{n}-\tilde{\mathcal{C}}_{n}^{0} & \equiv \int_{0}^{1} \mathrm{~d} t \frac{\mathrm{d}}{\mathrm{d} t} \tilde{\mathcal{C}}_{n}[\phi+(1-t) \varphi, \ldots ; t \varphi] \\
& =-\left.\varphi^{i} \int_{0}^{1} \mathrm{~d} t\left\{\left(\tau_{i} \tilde{\mathcal{C}}_{n}\right)[\underline{\phi}, \ldots ; \underline{\varphi}]\right\}\right|_{\substack{\phi \\
\underline{\varphi}=t \varphi}} .(1-t) \varphi
\end{aligned}
$$

Using the relation $\tau_{i} \mathcal{S}_{I_{0}} \tilde{\mathcal{C}}_{n}=0$ and the fact that $\left[\tau_{i}, \mathcal{S}_{I_{0}}\right]=0$, one concludes that the above difference belongs to the kernel of $\mathcal{S}_{I_{0}}$. In order to arrive at similar conclusions for the identities (A.4), (A.3) and (A.2) we have used arguments of [21]. For the remaining ones we have performed a "brute force" analysis of all possible terms in $\tilde{\mathcal{C}}_{n}$ consistent with the power-counting. Finally, for continuous global symmetries of $I_{0}$ Ward identities can be used, in parallel with (A.2) while for discrete symmetries $\tilde{\mathcal{C}}_{n}^{0}$ can be averaged over the group of discrete symmetries to obtain "new" $\tilde{\mathcal{C}}_{n}^{0}$ possessing discrete symmetries in question.

Obviously, $\tilde{\mathcal{C}}_{n}^{1} \in \operatorname{ker} \mathcal{S}_{I_{0}}$ can be discarded as far as restoration of the ZJ identity is concerned, cf. (4.17). In other words, for the counterterm restoring the BRST symmetry in the order $\hbar^{n+1}$ one can take

$$
\delta_{b} \Gamma_{n}^{(n+1)}=-\tilde{\mathcal{C}}_{n}^{0}
$$

preserving in this way the additional symmetries (A.1)-(A.8) of the next order local action $I_{n+1}$. This completes the inductive step.

\section{B One-loop diagrams}

Here we list the differences, defined in eq. (5.4), between the values of the one-loop diagrams in $\Lambda$ Reg and DimReg. They have been generated by a dedicated Mathematica based package in which the steps explained in section 3 have been implemented. These are 

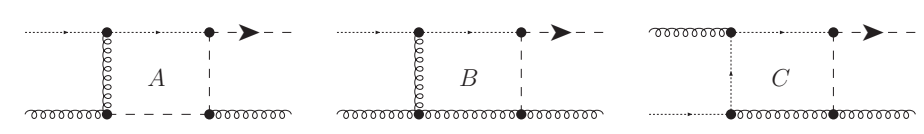

Figure 6. Function $\left\langle\tilde{K}_{i}(q) \tilde{\omega}^{\alpha}(l) \tilde{A}_{\nu}^{\beta}(p) \tilde{A}_{\rho}^{\gamma}\left(p^{\prime}\right)\right\rangle_{\widetilde{1 P I}}^{(1 B)}$.

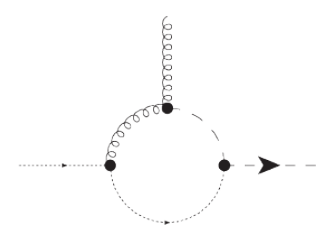

Figure 7. Function $\left\langle\tilde{K}_{i}(q) \tilde{\omega}^{\alpha}(l) \tilde{A}_{\nu}^{\beta}(p)\right\rangle_{\widetilde{1 P I}}^{(1 B)}$.

Figure 8. Corrections to the BRST transformation of scalars.

- expansion of regularized propagators according to (3.9),

- introduction of the Feynman parameters (at the level of tensor integrals),

- shift of the integration variable producing "spherically" symmetric denominators,

- expansion of the exponential factors in powers of external momenta,

- carrying out the integrations over angular variables in $d$ dimensions (making the standard replacements $k^{\mu} k^{\nu} \mapsto k^{2} \eta^{\mu \nu} / d$, etc.),

- transition to the Euclidean space (i.e. formal Wick rotation),

- contractions of tensor structures in $d$ dimensions. 


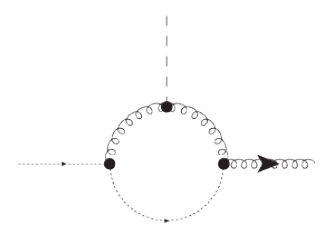

Figure 9. Function $\left\langle\tilde{K}_{\gamma}^{\mu}(q) \tilde{\omega}^{\alpha}(l) \tilde{\phi}^{j}(p)\right\rangle_{\widetilde{1 \mathrm{PI}}}^{(1 B)}$.

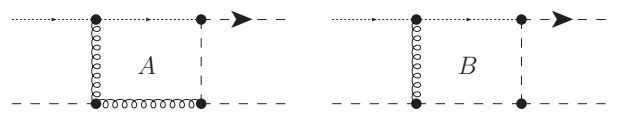

Figure 10. Function $\left\langle\tilde{K}_{n}(q) \tilde{\omega}^{\alpha}(l) \tilde{\phi}^{i}(p) \tilde{\phi}^{j}\left(p^{\prime}\right)\right\rangle_{\widetilde{1 P I}}^{(1)}$.

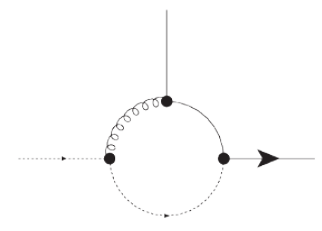

Figure 11. Corrections to the BRST transformation of fermions.

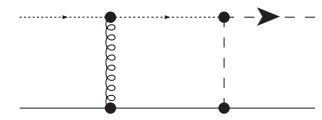

Figure 12. Function $\left\langle\tilde{K}_{i}(l) \tilde{\omega}^{\gamma}(q) \tilde{\psi}^{b_{1}}\left(p_{1}\right) \tilde{\psi}^{b_{2}}\left(p_{2}\right)\right\rangle_{\widetilde{1 P I}}^{(1)}$.

The expression corresponding to a one-loop diagram, obtained according to the above prescription, has the form of an integral over the Feynman parameters and over the length of the Euclidean momentum $k_{E}$. For $d \rightarrow 4$ it gives the value of the diagram in the $\Lambda$ Reg, while for $\Lambda \rightarrow \infty$ - in the DimReg. Starting from this point the package treats both cases separately. The expression corresponding to $\Lambda$ Reg is integrated over $k_{E}$ "algebraically", that is by exploiting the definition (3.10) of the confluent hypergeometric function. Analogous "algebraic" integration in the DimReg case exploits, instead of (3.10), the standard representation of the Euler beta function. Both are the Mathematica built-in functions (HypergeometricU and Beta, respectively) and their asymptotic forms can be found by calling the Series procedure. After the expansion nonlocal parts of both expressions manifestly cancel out in the difference, which becomes, therefore, a polynomial in the Feynman parameters which can be integrated over by using the Mathematica Integrate function. The package has been tested on many examples. In particular, we have verified that violations of the ST identities analyzed in sections 5.1-5.6, which were obtained by the direct calculation in $\Lambda$ Reg (of the type presented in $\mathrm{C}$ ), are reproduced by employing the trick discussed around eq. (5.4) using the formulae listed below.

Two remarks are in order. Firstly, the algorithm is simple, because the Feynman parameters are introduced at the level of tensor integrals. While this methods does yield 


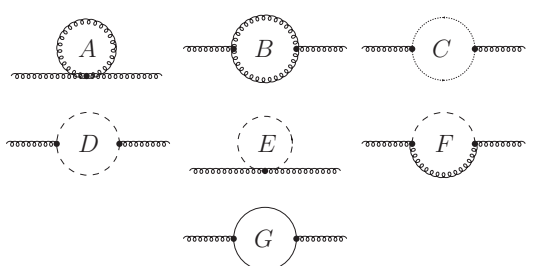

Figure 13. One-loop contributions to the vacuum polarization $\widetilde{\Gamma}_{\alpha \beta}^{\mu \nu}(l,-l)$.

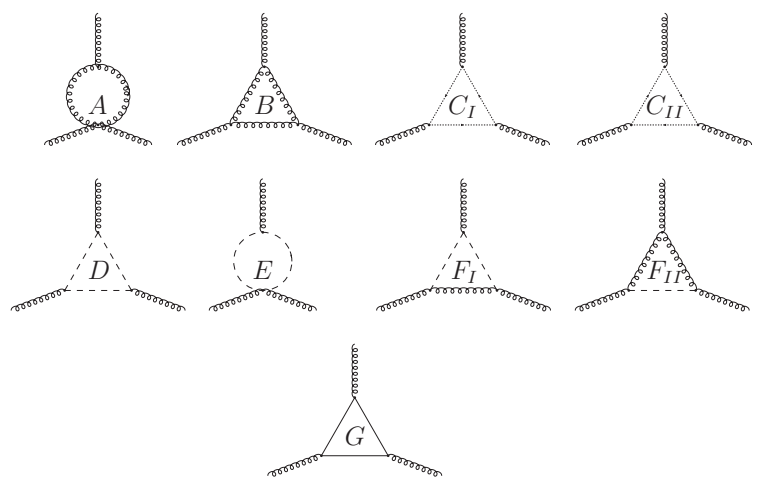

Figure 14. One-loop contributions to $\widetilde{\Gamma}_{\alpha \beta \gamma}^{\mu \nu \rho}\left(l, p, p^{\prime}\right)$.

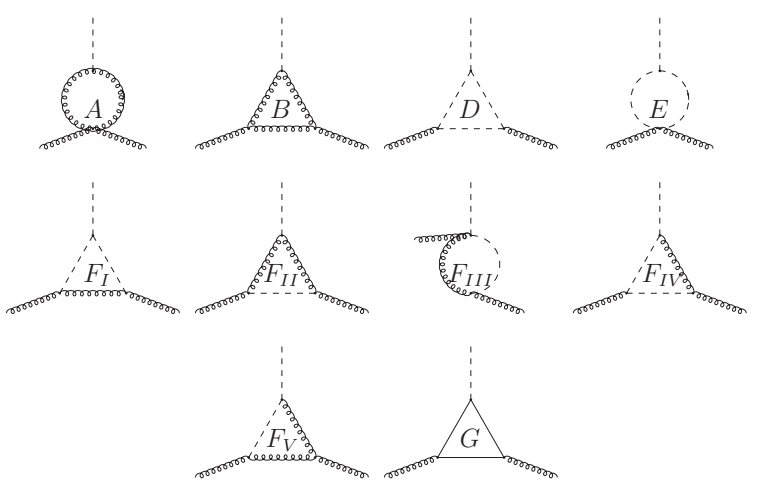

Figure 15. One-loop contributions to $\widetilde{\Gamma}_{i \beta \gamma}^{\nu \rho}\left(l, p, p^{\prime}\right)$.

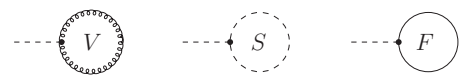

Figure 16. One-loop contributions to $\widetilde{\Gamma}_{i}$.

also expressions for the nonlocal parts of diagrams, their comparison with the result obtained with the help of the standard Passarino-Veltman reduction usually requires lengthy integrations by parts. Secondly, the package assumes that the $\varphi$-dependent mass matrices $m_{F}, m_{S}^{2}$ and $m_{V}^{2}$ are real and diagonal. These assumptions are satisfied only in a special basis in the field space, but results for the general case can always be unambiguously recovered. In particular, all the formulae given in this appendix and in the main text are correct for arbitrary mass matrices. 


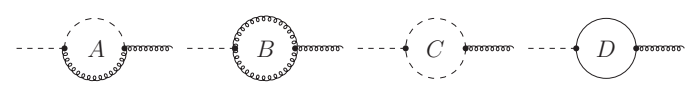

Figure 17. One-loop contributions to $\widetilde{\Gamma}_{i \beta}^{\nu}(l,-l)$.

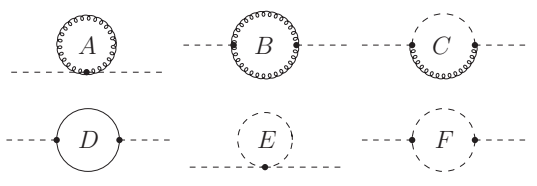

Figure 18. One-loop contribution to $\widetilde{\Gamma}_{i_{1} i_{2}}(p,-p)$.

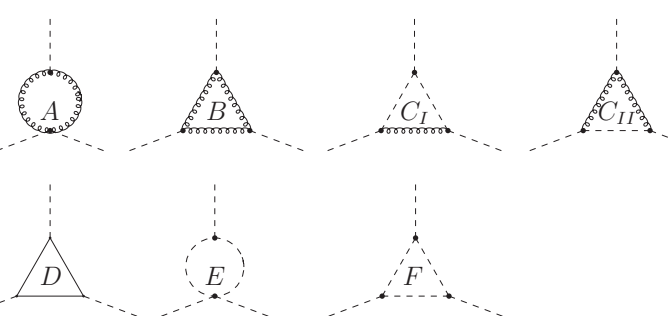

Figure 19. One loop contributions to $\widetilde{\Gamma}_{i j n}\left(p, p^{\prime}, p^{\prime \prime}\right)$.

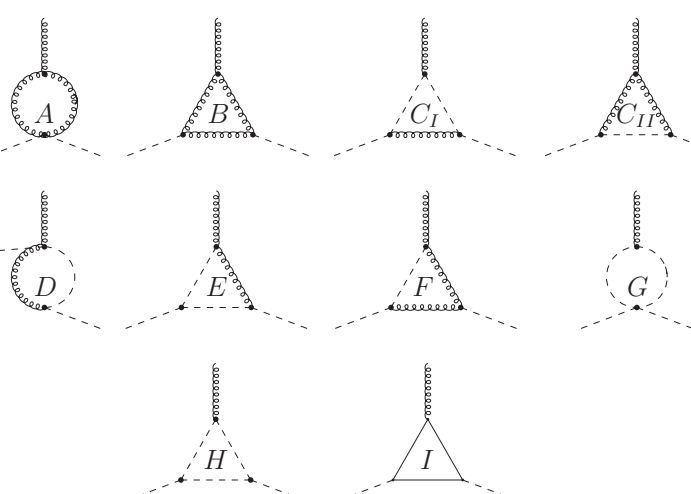

Figure 20. One-loop contributions to $\widetilde{\Gamma}_{i j \alpha}^{\mu}\left(p, p^{\prime}, l\right)$.

In the differences of the 1PI functions generated by the package one-loop logarithmic divergences always appear in the combination ${ }^{38}$

$$
\delta_{\text {Div }}=\ln \frac{\Lambda^{2}}{\mu_{H}^{2}}-\frac{1}{\epsilon}-1-\ln 8 \pi
$$

in which $\epsilon \equiv(4-d) / 2$ and $\mu_{H}$ is the 't Hooft mass - the natural mass unit of the DimReg (see e.g. the expression (B.4) below), which is also the renormalization scale of the ordinary DimReg-MS scheme. Since we are interested in renormalized parameters of the DimReg$\overline{\mathrm{MS}}$ scheme, it is more convenient to express $\delta_{\text {Div }}$ through the renormalization scale $\check{\mu}$ of

\footnotetext{
${ }^{38}$ This reflects the universality of one-loop logarithmic divergences which are related to the structure of non-local terms in the 1PI effective action.
} 


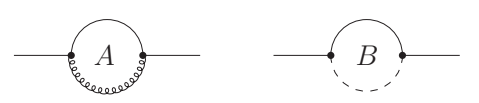

Figure 21. One-loop contributions to $\widetilde{\Gamma}_{a_{1} a_{2}}(p,-p)$.

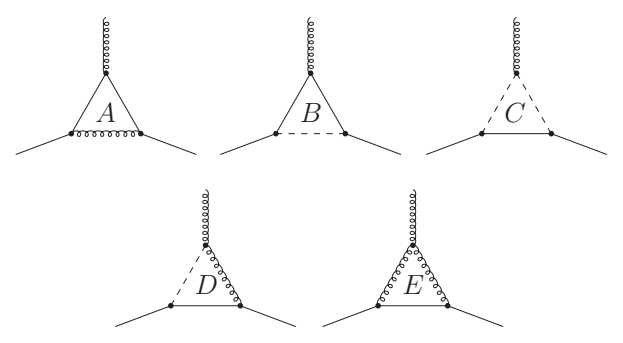

Figure 22. One-loop contributions to $\widetilde{\Gamma}_{a_{1} a_{2} \alpha}^{\mu}\left(p_{1}, p_{2}, q\right)$.

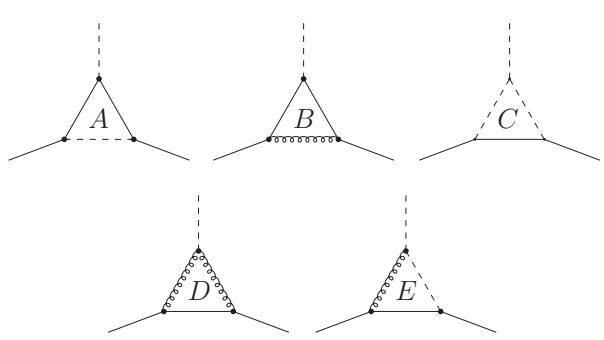

Figure 23. One-loop contributions to $\widetilde{\Gamma}_{a_{1} a_{2} i}\left(p_{1}, p_{2}, q\right)$.
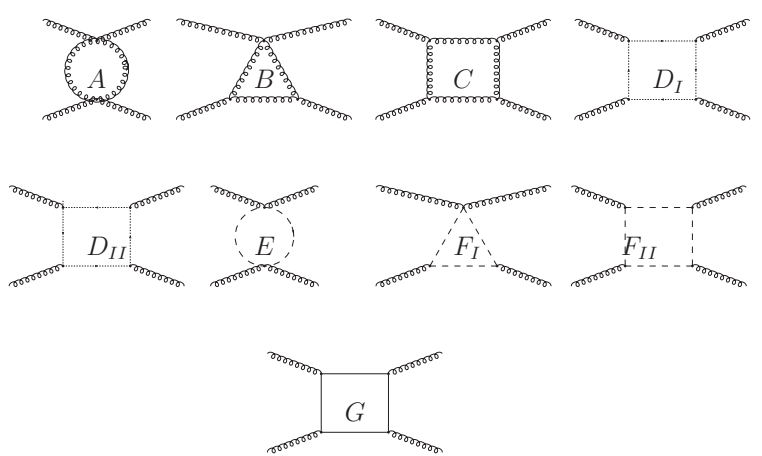

Figure 24. Regularization-dependent one-loop contributions to $\widetilde{\Gamma}_{\alpha_{1} \alpha_{2} \alpha_{3} \alpha_{4}}^{\mu_{1} \mu_{2} \mu_{3} \mu_{4}}\left(l_{1}, l_{2}, l_{3}, l_{4}\right)$.

the latter scheme which is related to $\mu_{H}$ by

$$
\check{\mu} \equiv \mu_{H} \sqrt{4 \pi} e^{-\gamma_{E} / 2},
$$

and the "fundamental divergence" $\delta_{\Lambda}$ of the $\Lambda$ - $\overline{\mathrm{MS}}$ scheme, defined in (4.13):

$$
\delta_{\text {Div }}=\delta_{\Lambda}-\frac{1}{\epsilon}-2 \ln \frac{\check{\mu}}{\mu} .
$$

It is clear that divergent parts of bare 1PI functions (in either regularization) can be easily recovered from the formulae listed below. 
To illustrate the method described above we quote here the explicit expression for the one-loop correction (diagram $C$ of figure 5) to the function $\left\langle K_{\alpha}^{\mu} \omega^{\gamma}\right\rangle$ entering all the ST identities analyzed in section 5

$$
\begin{aligned}
\left\langle\tilde{K}_{\alpha}^{\mu}(-q) \tilde{\omega}^{\gamma}(q)\right\rangle_{\overparen{1 \widetilde{P I}}}^{(1 B)}= & -i\left(e_{\beta} e_{\kappa}\right)_{\gamma}^{\alpha} \times \\
& \times \mu_{H}^{4-d} \int \frac{\mathrm{d}^{d} k}{(2 \pi)^{d}}\left[\frac{i}{\mathcal{R}(k)^{2}-m_{V}^{2}}\right]^{\beta \kappa}\left[\eta_{\mu \nu}-\frac{k_{\mu} k_{\nu}}{k^{2}}\right] \frac{\mathcal{R}^{\nu}(k+q)}{\mathcal{R}(k+q)^{2}}
\end{aligned}
$$

(In diagrams external lines of antifields are marked in the same way as those of the corresponding fields but carry the extra arrow pointing the direction of the flow of the ghost number). For $d=4$ the integral in (B.4) is regularized according to the prescription (3.2), whereas for $\Lambda=\infty$ it is regularized dimensionally. For the difference defined in (5.4), omitting terms which vanish in the limits $\Lambda \rightarrow \infty, \epsilon \rightarrow 0$, one gets

$$
\Delta\left\langle\tilde{K}_{\kappa}^{\sigma}(-l) \tilde{\omega}^{\alpha}(l)\right\rangle_{\widetilde{1 P I}}^{(1 B)}=\frac{i l_{\sigma}}{(4 \pi)^{2}}\left(e_{\gamma} e^{\gamma}\right)_{\alpha}^{\kappa}\left(\frac{1}{8}+\frac{3}{4} \ln \frac{4}{3}+\frac{3}{4} \delta_{\text {Div }}\right) .
$$

As to the other 1PI functions of antifields, there are no one-loop diagrams contributing to the function $\left\langle\tilde{K}_{i}(q) \tilde{\omega}^{\alpha}(l)\right\rangle_{\widetilde{1 P I}}^{(1 B)}$. To the function $\left\langle\tilde{K}_{\kappa}^{\sigma}\left(p^{\prime}\right) \tilde{\omega}^{\alpha}(l) \tilde{A}_{\nu}^{\beta}(p)\right\rangle_{\widetilde{1 P I}}^{(1 B)}$ contribute the diagrams $A$ and $B$ shown in figure 5 . However, owing to the antighost equation (A.5) both these contributions are independent of the regularization (despite being superficially logarithmically divergent). Likewise diagrams with external lines of antifields, shown in figures $6-12$, which contribute to the following functions of the antifields

$$
\begin{array}{lll}
\left\langle\tilde{K}_{i}(q) \tilde{\omega}^{\alpha}(l)\right\rangle_{\widetilde{\mathrm{PI}}}^{(1 B)}, & \left\langle\tilde{K}_{\kappa}^{\sigma}\left(p^{\prime}\right) \tilde{\omega}^{\alpha}(l) \tilde{A}_{\nu}^{\beta}(p)\right\rangle_{\widetilde{1 \mathrm{PI}}}^{(1 B)}, & \left\langle\tilde{K}_{i}\left(p^{\prime}\right) \tilde{\omega}^{\alpha}(l) \tilde{A}_{\nu}^{\beta}(p)\right\rangle_{\widetilde{1 \mathrm{PI}}}^{(1 B)}, \\
\left\langle\tilde{K}_{i}(q) \tilde{\omega}^{\alpha}(l) \tilde{A}_{\nu}^{\beta}(p) \tilde{A}_{\rho}^{\gamma}\left(p^{\prime}\right)\right\rangle_{\widetilde{1 \mathrm{PI}}}^{(1 B)}, & \left\langle\tilde{K}_{n}(r) \tilde{\omega}^{\alpha}(l) \tilde{\phi}^{i}(p)\right\rangle_{\widetilde{1 P I}}^{(1 B)}, & \left\langle\tilde{K}_{\gamma}^{\mu}(q) \tilde{\omega}^{\alpha}(l) \tilde{\phi}^{j}(p)\right\rangle_{\widetilde{\mathrm{PI}}}^{(1 B)}, \\
\left\langle\tilde{K}_{n}(q) \tilde{\omega}^{\alpha}(l) \tilde{\phi}^{i}(p) \tilde{\phi}^{j}\left(p^{\prime}\right)\right\rangle_{\widetilde{\mathrm{IPI}}}^{(1)}, & \left\langle\tilde{K}_{n}(l) \tilde{\omega}^{\gamma}(q) \tilde{\psi}^{b_{1}}\left(p_{1}\right) \tilde{\psi}^{b_{2}}\left(p_{2}\right)\right\rangle_{\widetilde{1 \mathrm{PI}}}^{(1)}, & \\
\left\langle\tilde{K}_{a_{1}}\left(p_{1}\right) \tilde{\omega}^{\gamma}(q) \tilde{\psi}^{a_{2}}\left(p_{2}\right)\right\rangle_{\widetilde{\mathrm{PI}}}^{(1 B)} & &
\end{array}
$$

are independent of regularization. Therefore, the differences (5.4) corresponding to these function vanish.

One-loop diagrams contributing to the two-point function $\widetilde{\Gamma}_{\alpha \beta}^{\mu \nu}$ are shown in figure 13. The corresponding difference (5.4) reads

$$
\begin{gathered}
(4 \pi)^{2} \Delta \widetilde{\Gamma}_{\alpha \beta}^{\mu \nu}(p,-p)^{(1 B)}=\operatorname{tr}\left(e_{\alpha} e_{\beta} m_{V}^{2}\right) \eta^{\mu \nu}\left(\frac{11}{8}-\frac{3}{4} \delta_{\text {Div }}\right)+ \\
+\operatorname{tr}\left(e_{\alpha} e_{\beta}\right)\left[p^{\mu} p^{\nu}\left(\frac{1}{9}+\frac{13}{6} \delta_{\text {Div }}+\frac{19}{6} \ln 2-\frac{3}{2} \ln 3\right)+\right. \\
\left.+\eta^{\mu \nu}\left(-\frac{23}{72} p^{2}-\frac{13}{6} p^{2} \delta_{\text {Div }}-\frac{19}{6} p^{2} \ln 2+\frac{3}{2} p^{2} \ln 3-\Lambda^{2}\right)\right]+ \\
+\operatorname{tr}\left(\mathcal{T}_{\alpha} \mathcal{T}_{\beta}\right)\left[p^{\mu} p^{\nu}\left(-\frac{11}{72}-\frac{1}{6} \delta_{\text {Div }}-\frac{1}{6} \ln 2\right)+\right.
\end{gathered}
$$




$$
\begin{gathered}
\left.+\eta^{\mu \nu}\left(\frac{7}{144} p^{2}+\frac{1}{6} p^{2} \delta_{\text {Div }}+\frac{1}{12} p^{2} \ln 4-\frac{\Lambda^{2}}{2}\right)\right]+ \\
+\frac{1}{4} \eta^{\mu \nu} \operatorname{tr}\left(\left\{\mathcal{T}_{\alpha}, \mathcal{T}_{\beta}\right\} m_{S}^{2}\right)-\left\{\frac{1}{8}+\frac{3}{4} \delta_{\text {Div }}\right\} \eta^{\mu \nu} \varphi^{\mathrm{T}}\left\{\mathcal{T}_{\alpha}, \mathcal{T}_{\epsilon}\right\}\left\{\mathcal{T}^{\epsilon}, \mathcal{T}_{\beta}\right\} \varphi+ \\
-\operatorname{tr}\left(\mathfrak{f}_{\alpha} \mathfrak{f}_{\beta}\right)\left[p^{\mu} p^{\nu}\left(\frac{5}{18}+\frac{2}{3} \delta_{\text {Div }}+\frac{2}{3} \ln 2\right)+\right. \\
\left.-\eta^{\mu \nu}\left(\Lambda^{2}+\frac{11}{18} p^{2}+\frac{2}{3} p^{2} \delta_{\text {Div }}+\frac{2}{3} p^{2} \ln 2\right)\right]+ \\
+\eta^{\mu \nu}\left[2 \delta_{\text {Div }} \operatorname{tr}\left(\mathfrak{f}_{\alpha} m_{F}^{*} \mathfrak{f}_{\beta}^{*} m_{F}\right)-\left(\delta_{\text {Div }}+\frac{1}{2}\right) \operatorname{tr}\left(\left\{\mathfrak{f}_{\alpha}, \mathfrak{f}_{\beta}\right\} m_{F}^{*} m_{F}\right)\right] .
\end{gathered}
$$

One-loop corrections to the three-point function $\widetilde{\Gamma}_{\alpha \beta \gamma}^{\mu \nu \rho}\left(l, p, p^{\prime}\right)$ are displayed in figure 14. For fermions in a non-anomalous representation the corresponding difference (5.4) (diagrams $E, F_{I}$ and $F_{I I}$ do not contribute to it) reads

$$
\begin{aligned}
& (4 \pi)^{2} \Delta \widetilde{\Gamma}_{\alpha \beta \gamma}^{\mu \nu \rho}\left(l, p, p^{\prime}\right)^{(1 B)}=\left[\eta^{\mu \rho}\left(l-p^{\prime}\right)^{\nu}+\eta^{\mu \nu}(p-l)^{\rho}+\eta^{\nu \rho}\left(p^{\prime}-p\right)^{\mu}\right] \times \\
& \times\left\{i \operatorname{tr}\left(e_{\alpha} e_{\beta} e_{\gamma}\right)\left(\frac{11}{12}+\frac{17}{6} \delta_{\text {Div }}+\frac{4}{3} \ln \frac{4}{3}\right)-\frac{i}{6} \operatorname{tr}\left(\mathcal{T}_{\alpha} \mathcal{T}_{\beta} \mathcal{T}_{\gamma}\right)\left(-1+2 \delta_{\text {Div }}+2 \ln \frac{4}{3}\right)+\right. \\
& \left.\quad-\frac{i}{3} \operatorname{tr}\left(\mathfrak{f}_{\alpha}\left[\mathfrak{f}_{\beta}, \mathfrak{f}_{\gamma}\right]\right)\left(1+2 \delta_{\text {Div }}+2 \ln \frac{4}{3}\right)\right\}
\end{aligned}
$$

Diagrams contributing to the function $\langle\phi A A\rangle$ are shown in figure 15 (the diagram $C$ is not shown, because ghosts do not couple to scalars in the Landau gauge). Power-counting, the Lorentz symmetry and the translational invariance (3.3) of the effective action imply the relation

$$
\Delta \widetilde{\Gamma}_{i \beta \gamma}^{\nu \rho}\left(l, p, p^{\prime}\right)^{(1 B)}=\Delta \widetilde{\Gamma}_{i \beta \gamma}^{\nu \rho}(0, p,-p)^{(1 B)}=\frac{\partial}{\partial \varphi^{i}} \Delta \widetilde{\Gamma}_{\beta \gamma}^{\nu \rho}(p,-p)^{(1 B)}
$$

between (B.6) and $\Delta \widetilde{\Gamma}_{i \beta \gamma}^{\nu \rho}\left(l, p, p^{\prime}\right)^{(1 B)}$. By computing the latter difference directly we have checked that the relation (B.8) does indeed hold.

The difference (5.4) corresponding to the one-loop tadpole diagrams shown in figure 16 is

$$
\begin{gathered}
(4 \pi)^{2} \Delta \widetilde{\Gamma}_{i}(p)^{(1 B)}=\Lambda^{2} \operatorname{tr}\left\{Y_{i} m_{F}^{*}+c c .\right\}-\delta_{\text {Div }} \operatorname{tr}\left\{Y_{i} m_{F}^{*} m_{F} m_{F}^{*}+c c .\right\}-\frac{1}{2} \Lambda^{2} \operatorname{tr}\left\{\rho_{i}\right\}+ \\
+\frac{1}{2} \delta_{\text {Div }} \operatorname{tr}\left\{\rho_{i} m_{S}^{2}\right\}+\frac{1}{2}\left[3 \Lambda^{2} \delta_{\alpha \beta}-\left(3 \delta_{\text {Div }}+2\right) m_{V \alpha \beta}^{2}\right]\left(\varphi^{\mathrm{T}}\left\{\mathcal{T}^{\alpha}, \mathcal{T}^{\beta}\right\}\right)_{i} .
\end{gathered}
$$

(The couplings $\rho_{i j k}$ and the scalar fields mass matrix $m_{S}^{2}$ are defined in (2.2)).

Diagrams contributing to the $\langle\phi A\rangle$ function are presented in figure 17 (diagrams $B$ and $C$ have the same value in both regularizations). The corresponding difference (5.4) reads

$$
\begin{aligned}
(4 \pi)^{2} \Delta & \widetilde{\Gamma}_{i \beta}^{\nu}(l,-l)^{(1 B)}=-i l^{\nu}\left(\frac{1}{4}+\frac{3}{2} \delta_{\text {Div }}-\frac{3}{4} \ln \frac{3}{4}\right)\left(\mathcal{T}^{\kappa}\left\{\mathcal{T}_{\kappa}, \mathcal{T}_{\beta}\right\} \varphi\right)_{i}+ \\
& +i l^{\nu}\left(\frac{1}{12}+\frac{1}{2} \delta_{\text {Div }}-\frac{1}{2} \ln \frac{3}{4}\right) \operatorname{tr}\left(Y_{i} m_{F}^{*} \mathfrak{f}_{\beta}^{*}-Y_{i} \mathfrak{f}_{\beta} m_{F}^{*}+Y_{i}^{*} m_{F} \mathfrak{f}_{\beta}-Y_{i}^{*} \mathfrak{f}_{\beta}^{*} m_{F}\right) .
\end{aligned}
$$


Diagrams contributing to the function $\langle\phi \phi\rangle$ are shown in figure 18. They give

$$
\begin{aligned}
& (4 \pi)^{2} \Delta \widetilde{\Gamma}_{i_{1} i_{2}}(l,-l)^{(1 B)}=3 \Lambda^{2}\left(\mathcal{T}^{\alpha} \mathcal{T}_{\alpha}\right)_{i_{1} i_{2}}+ \\
& \quad-\left[\frac{3}{2} \delta_{\text {Div }}+1\right]\left[\left(m_{V}^{2}\right)_{\alpha \beta}\left\{\mathcal{T}^{\alpha}, \mathcal{T}^{\beta}\right\}_{i_{1} i_{2}}-\left(\left\{\mathcal{T}^{\alpha}, \mathcal{T}^{\beta}\right\} \varphi\right)_{i_{1}}\left(\left\{\mathcal{T}_{\alpha}, \mathcal{T}_{\beta}\right\} \varphi\right)_{i_{2}}\right]+ \\
& \quad+l^{2}\left(3 \delta_{\text {Div }}+\frac{1}{2}+\frac{3}{4} \ln \frac{32}{9}\right)\left(\mathcal{T}^{\alpha} \mathcal{T}_{\alpha}\right)_{i_{1} i_{2}}+ \\
& \quad+\left[\Lambda^{2}+l^{2}\left(\frac{1}{2} \delta_{\text {Div }}+\frac{3}{8}+\frac{1}{2} \ln 2\right)\right] \operatorname{tr}\left[Y_{i_{1}} Y_{i_{2}}^{*}+c c .\right]+ \\
& \quad-\delta_{\text {Div }} \operatorname{tr}\left[\left(Y_{i_{1}} m_{F}^{*} m_{F} Y_{i_{2}}^{*}+Y_{i_{1}} Y_{i_{2}}^{*} m_{F} m_{F}^{*}+Y_{i_{1}} m_{F}^{*} Y_{i_{2}} m_{F}^{*}\right)+c c .\right]+ \\
& \quad-\frac{1}{2} \lambda_{i_{1} i_{2} j_{1} j_{2}}\left[\delta^{j_{1} j_{2}} \Lambda^{2}-\delta_{\text {Div }}\left(m_{S}^{2}\right)^{j_{1} j_{2}}\right]+\frac{1}{2} \delta_{\text {Div }} \operatorname{tr}\left(\rho_{i_{1}} \rho_{i_{2}}\right) .
\end{aligned}
$$

Contributions to the $\langle\phi \phi \phi\rangle$ vertex are displayed in figure 19 (only diagrams $A, D$ and $E$ are different in $\Lambda$ Reg and DimReg). Since both regularizations preserve (3.3), $\Delta \tilde{\Gamma}_{i_{1} i_{2} i_{3}}^{(1 B)}$ obtained by direct calculation coincides with the result of differentiating $\Delta \widetilde{\Gamma}_{i_{1} i_{2}}(l,-l)^{(1 B)}$ given in (B.11) with respect to the background $\varphi$.

Only diagrams $D$ and $I$ of figure 20 contribute to the difference

$$
\begin{aligned}
& (4 \pi)^{2} \Delta \widetilde{\Gamma}_{i j \alpha}^{\mu}\left(p, p^{\prime}, l\right)^{(1 B)}=i\left(p^{\mu}-p^{\prime \mu}\right) \times \\
& \quad \times\left\{\left[\frac{1}{6}+\delta_{\text {Div }}-\ln \frac{3}{4}\right] \operatorname{tr}\left[Y_{i}^{*} Y_{j} \mathfrak{f}_{\alpha}+c c .\right]-\left[\frac{1}{4}+\frac{3}{2} \delta_{\text {Div }}-\frac{3}{4} \ln \frac{3}{4}\right]\left(\mathcal{T}_{\beta}\left\{\mathcal{T}_{\alpha}, \mathcal{T}^{\beta}\right\}\right)_{i j}\right\} .
\end{aligned}
$$

corresponding to the $\langle\phi \phi A\rangle$ vertex.

For 1PI functions with two external fermionic lines (recall that we work with Majorana fermions) we use the matrix notation in which spinor indices are omitted; we write for example

$$
\widetilde{\Gamma}_{\psi \otimes \psi}\left(p_{1}, p_{2}\right) \equiv\left[\widetilde{\Gamma}_{a_{1} a_{2}}\left(p_{1}, p_{2}, q\right)\right], \quad \widetilde{\Gamma}_{\psi \otimes \psi \alpha}^{\mu}\left(p_{1}, p_{2}, q\right) \equiv\left[\widetilde{\Gamma}_{a_{1} a_{2} \alpha}^{\mu}\left(p_{1}, p_{2}, q\right)\right],
$$

etc. In this notation the diagrams of figure 21 give

$$
\begin{aligned}
(4 \pi)^{2} \Delta \widetilde{\Gamma}_{\psi \otimes \psi}(p,-p)^{(1 B)}= & C \not p\left\{t_{\gamma} t^{\gamma}+\frac{1}{2}\left[\ln \frac{3}{4}-\frac{1}{6}-\delta_{\text {Div }}\right] y_{i}^{*} y^{i}\right\}+ \\
& -\left(3 \delta_{\text {Div }}+2\right) C t_{\gamma}^{\mathrm{T}} \widehat{m}_{F} t^{\gamma}+\delta_{\text {Div }} C y_{i} \widehat{m}_{F}^{*} y^{i} .
\end{aligned}
$$

for the difference (5.4) of the corresponding $\langle\psi \psi\rangle$ functions in the two schemes,

$$
\begin{aligned}
(4 \pi)^{2} \Delta \widetilde{\Gamma}_{\psi \otimes \psi \alpha}^{\mu}\left(p_{1}, p_{2}, q\right)^{(1 B)}=i C & \left\{-\frac{3}{2} \gamma^{\mu} t_{\kappa} t_{\alpha} t^{\kappa}+\frac{1}{4}\left(2 \delta_{\text {Div }}+1\right) y^{i} t_{\alpha} y_{i}^{*} \gamma^{\mu}+\right. \\
& \left.+\frac{1}{4}\left(2 \delta_{\text {Div }}-1\right) \gamma^{\mu} y_{i}^{*} y_{j} \mathcal{T}_{\alpha}^{i j}+\frac{3}{2}\left[\delta_{\text {Div }}+\frac{1}{6}\right] \gamma^{\mu} t_{\kappa} t_{\beta} e_{\alpha \beta}^{\kappa \beta}\right\} .
\end{aligned}
$$

for the difference of the $\langle\psi \psi A\rangle$ vertices (diagrams of figure 22) and

$$
(4 \pi)^{2} \Delta \widetilde{\Gamma}_{\psi \otimes \psi i}\left(p_{1}, p_{2}, q\right)^{(1 B)}=C\left\{-\left(3 \delta_{\text {Div }}+2\right) t_{\gamma}^{\mathrm{T}} y_{i} t^{\gamma}+\delta_{\text {Div }} y_{j} y_{i}^{*} y^{j}\right\} .
$$


for the difference of the $\langle\psi \psi \phi\rangle$ vertices (diagrams of figure 23). As expected, (B.15) is just the derivative of (B.13) w.r.t. $\varphi$.

Regularization-dependent contributions to the four-point function $\langle A A A A\rangle$ are shown in figure 24. The corresponding difference (5.4) has (for non-anomalous representations $\mathfrak{f}_{\alpha}$ ) the unambiguous form

$$
\begin{aligned}
& (4 \pi)^{2} \Delta \widetilde{\Gamma}_{\alpha_{1} \alpha_{2} \alpha_{3} \alpha_{4}}^{\mu_{1} \mu_{2} \mu_{3} \mu_{4}}\left(l_{1}, l_{2}, l_{3}, l_{4}\right)^{(1 B)}= \\
& =\eta^{\mu_{1} \mu_{2}} \eta^{\mu_{3} \mu_{4}} \mathbb{V}_{\alpha_{1} \alpha_{2} \alpha_{3} \alpha_{4}}+\eta^{\mu_{1} \mu_{4}} \eta^{\mu_{3} \mu_{2}} \mathbb{V}_{\alpha_{1} \alpha_{4} \alpha_{3} \alpha_{2}}+\eta^{\mu_{1} \mu_{3}} \eta^{\mu_{2} \mu_{4}} \mathbb{V}_{\alpha_{1} \alpha_{3} \alpha_{2} \alpha_{4}}
\end{aligned}
$$

where

$$
\begin{aligned}
\mathbb{V}_{\alpha_{1} \alpha_{2} \alpha_{3} \alpha_{4}}= & \frac{1}{9}\left\{\left(11+12 \delta_{\text {Div }}\right) \operatorname{tr}\left(e_{\alpha_{1}} e_{\alpha_{2}}\left\{e_{\alpha_{3}}, e_{\alpha_{4}}\right\}\right)-8\left(2+3 \delta_{\text {Div }}\right) \operatorname{tr}\left(e_{\alpha_{1}} e_{\alpha_{3}} e_{\alpha_{2}} e_{\alpha_{4}}\right)\right\}+ \\
+ & \frac{1}{6}\left\{\left(\delta_{\text {Div }}-1\right) \operatorname{tr}\left(\left[\mathcal{T}_{\alpha_{2}}, \mathcal{T}_{\alpha_{3}}\right]\left[\mathcal{T}_{\alpha_{1}}, \mathcal{T}_{\alpha_{4}}\right]+\left[\mathcal{T}_{\alpha_{1}}, \mathcal{T}_{\alpha_{3}}\right]\left[\mathcal{T}_{\alpha_{2}}, \mathcal{T}_{\alpha_{4}}\right]\right)+\right. \\
& \left.\quad+2 \operatorname{tr}\left(\mathcal{T}_{\left(\alpha_{1}\right.} \mathcal{T}_{\alpha_{2}} \mathcal{T}_{\alpha_{3}} \mathcal{T}_{\left.\alpha_{4}\right)}\right)\right\}+ \\
& -\frac{2}{3} \delta_{\text {Div }} \operatorname{tr}\left(\left[\mathfrak{f}_{\alpha_{4}}, \mathfrak{f}_{\alpha_{1}}\right]\left[\mathfrak{f}_{\alpha_{2}}, \mathfrak{f}_{\alpha_{3}}\right]+\left[\mathfrak{f}_{\alpha_{1}}, \mathfrak{f}_{\alpha_{3}}\right]\left[\mathfrak{f}_{\alpha_{4}}, \mathfrak{f}_{\alpha_{2}}\right]\right)+ \\
& -\frac{4}{9} \operatorname{tr}\left(\left\{\mathfrak{f}_{\alpha_{1}}, \mathfrak{f}_{\alpha_{2}}\right\}\left\{\mathfrak{f}_{\alpha_{3}}, \mathfrak{f}_{\alpha_{4}}\right\}\right)+\frac{5}{9} \operatorname{tr}\left(\mathfrak{f}_{\alpha_{1}} \mathfrak{f}_{\alpha_{3}} \mathfrak{f}_{\alpha_{2}} \mathfrak{f}_{\alpha_{4}}+\mathfrak{f}_{\alpha_{3}} \mathfrak{f}_{\alpha_{1}} \mathfrak{f}_{\alpha_{4}} \mathfrak{f}_{\alpha_{2}}\right),
\end{aligned}
$$

(notice the symmetrization of the indices $\alpha_{1}, \ldots, \alpha_{4}$ in the third line). Divergences are associated only with the structure constants as expected. In general (i.e. not assuming (4.10)) the difference (B.16) would contain also terms proportional to the Levi-Civita tensor $\epsilon^{\alpha_{1} \alpha_{2} \alpha_{3} \alpha_{4}}$ which cannot be determined uniquely because of the ambiguities of the DimReg scheme with the naive $\gamma^{5}$. Such terms are multiplied by tensors of the form

$$
\operatorname{tr}\left(\mathfrak{f}_{\alpha_{1}} \mathfrak{f}_{\alpha_{2}} \mathfrak{f}_{\alpha_{3}} \mathfrak{f}_{\alpha_{4}}-c c .\right) \equiv \frac{1}{2} \operatorname{tr}\left(\left\{\mathfrak{f}_{\alpha_{1}}, \mathfrak{f}_{\alpha_{2}}\right\}\left[\mathfrak{f}_{\alpha_{3}}, \mathfrak{f}_{\alpha_{4}}\right]\right)+\frac{1}{2} \operatorname{tr}\left(\left[\mathfrak{f}_{\alpha_{1}}, \mathfrak{f}_{\alpha_{2}}\right]\left\{\mathfrak{f}_{\alpha_{3}}, \mathfrak{f}_{\alpha_{4}}\right\}\right)
$$

and vanish for a non-anomalous fermionic representation.

\section{Chiral anomaly}

In this appendix we determine directly the contribution of fermionic loops to the ST identity (5.38) involving the $\langle A A A A\rangle$ vertex. We use the notation introduced in subsection 5.7 (eqs. (5.36) and (5.37)).

Fermionic loops contribute only to the first three terms of the l.h.s. of the identity (5.38). Since it potentially can have a true anomaly, it will be instructive to generalize the regularization $(3.2)$ by not specifying explicitly the profile $g(\cdot)$ of the function entering the regularization prescription

$$
k^{\mu} \rightarrow \mathcal{R}^{\mu}(k)=\frac{1}{\tilde{g}(k)} \times k^{\mu}, \quad \tilde{g}(k) \equiv g\left(k^{2} / \Lambda^{2}\right),
$$

but assuming only that $g$ is an analytic function satisfying the boundary conditions

$$
g(0)=1, \quad g(x) \stackrel{x \rightarrow-\infty}{\longrightarrow} 0 .
$$


We also use the following notation for fermionic propagators (cf. eq. (3.4)):

$$
\mathscr{S}_{\Lambda}(k) \equiv S(k ; \Lambda) C, \quad \mathscr{S}(k) \equiv \lim _{\Lambda \rightarrow \infty} \mathscr{S}_{\Lambda}(k) .
$$

The contribution of fermions to the $\langle A A A A\rangle$ vertex (diagram $G$ of figure 24) reads

$$
\widetilde{\Gamma}_{\alpha_{1} \alpha_{2} \alpha_{3} \alpha_{4}}^{\mu_{1} \mu_{2} \mu_{3} \mu_{4}}\left(l_{1}, l_{2}, l_{3}, l_{4} \mid G\right) \equiv \mathscr{G}_{\imath_{1} \imath_{2} \imath_{3} \imath_{4}}=\mathbb{G}_{\imath_{1} \imath_{2} \imath_{3} \imath_{4}}+\mathbb{G}_{\imath_{1} \imath_{2} \imath_{4} \imath_{3}}+\mathbb{G}_{\imath_{1} \imath_{3} \imath_{2} \imath_{4}},
$$

where

$$
\begin{aligned}
& \mathbb{G}_{\imath_{1} \imath_{2} \imath_{3} \imath_{4}} \\
& \quad=\operatorname{tr} \int \frac{\mathrm{d}^{4} k}{(2 \pi)^{4}} i \gamma^{\mu_{1}} t_{\alpha_{1}} \mathscr{S}_{\Lambda}(k) \gamma^{\mu_{2}} t_{\alpha_{2}} \mathscr{S}_{\Lambda}\left(k-l_{2}\right) \gamma^{\mu_{3}} t_{\alpha_{3}} \mathscr{S}_{\Lambda}\left(k-l_{2}-l_{3}\right) \gamma^{\mu_{4}} t_{\alpha_{4}} \mathscr{S}_{\Lambda}\left(k+l_{1}\right),
\end{aligned}
$$

while the analogous contribution to the $\langle\phi A A A\rangle$ vertex has the form

$$
\widetilde{\Gamma}_{j \alpha_{2} \alpha_{3} \alpha_{4}}^{\mu_{2} \mu_{3} \mu_{4}}\left(l_{1}, l_{2}, l_{3}, l_{4} \mid G\right) \equiv \overline{\mathbb{G}}_{j \imath_{2} \imath_{3} \imath_{4}}+\overline{\mathbb{G}}_{j \imath_{2} \imath_{4} \imath_{3}}+\overline{\mathbb{G}}_{j \imath_{3} \imath_{2} \imath_{4}},
$$

with

$$
\begin{aligned}
& \overline{\mathbb{G}}_{\mathfrak{j}_{2} \imath_{3} \imath_{4}} \\
& \quad=i \operatorname{tr} \int \frac{\mathrm{d}^{4} k}{(2 \pi)^{4}} i y_{j} \mathscr{S}_{\Lambda}(k) \gamma^{\mu_{2}} t_{\alpha_{2}} \mathscr{S}_{\Lambda}\left(k-l_{2}\right) \gamma^{\mu_{3}} t_{\alpha_{3}} \mathscr{S}_{\Lambda}\left(k-l_{2}-l_{3}\right) \gamma^{\mu_{4}} t_{\alpha_{4}} \mathscr{S}_{\Lambda}\left(k+l_{1}\right) .
\end{aligned}
$$

Finally the diagram $G$ of figure 14 gives

$$
\widetilde{\Gamma}_{\alpha \beta \gamma}^{\mu \nu \rho}\left(l, p, p^{\prime} \mid G\right)=i \operatorname{tr} \int \frac{\mathrm{d}^{4} k}{(2 \pi)^{4}} i \gamma^{\nu} t_{\beta} \mathscr{S}_{\Lambda}(k) \gamma^{\rho} t_{\gamma} \mathscr{S}_{\Lambda}\left(k-p^{\prime}\right) \gamma^{\mu} t_{\alpha} \mathscr{S}_{\Lambda}(k+p) .
$$

The total contribution of these three functions to the l.h.s. of (5.38) will be denoted $\boldsymbol{\Omega}_{4 F}$. To simplify it, we decompose the contribution of (C.4) contracted with the momentum $l_{1}^{\mu_{1}}$ using the identity

$$
l_{1} t_{\alpha_{1}}=\left\{\not k+\not l_{1}-\widehat{m}_{F}\right\} t_{\alpha_{1}}+t_{\alpha_{1}}^{\mathrm{T}}\left\{\not k-\widehat{m}_{F}\right\}-\left(\mathcal{T}_{\alpha_{1}} \varphi\right)^{j} y_{j},
$$

(following from the gauge symmetry of $I_{0}^{G I}$ ) into three parts. The one that originates from the last term of (C.9) cancels exactly in $\boldsymbol{\Omega}_{4 F}$ the contribution of (C.6). Furthermore, after expanding the propagators in the remaining terms of $\boldsymbol{\Omega}_{4 F}$ according to (3.9) and retaining only those integrals that do not vanish in the limit $\Lambda \rightarrow \infty$, "similar" terms ${ }^{39}$ can be combined. As a result of these operations $\boldsymbol{\Omega}_{4 F}$ can be represented in the form

$$
\boldsymbol{\Omega}_{4 F}=\left(\mathbb{X}^{I}+\mathbb{X}^{I I}\right)_{\imath_{1} \imath_{2} \imath_{3} \imath_{4}}+\left(\mathbb{X}^{I}+\mathbb{X}^{I I}\right)_{\imath_{1} \imath_{4} \imath_{3} \imath_{2}}+\left(\mathbb{X}^{I}+\mathbb{X}^{I I}\right)_{\imath_{1} \imath_{2} \imath_{4} \imath_{3}}+\mathcal{O}\left(\Lambda^{-1}\right),
$$

\footnotetext{
${ }^{39}$ Some of these "similarities" become visible only after shifting the integration momentum and replacing the matrix under the trace by its transposition, with the aid of standard relations

$$
C \gamma^{\mu \mathrm{T}} C=\gamma^{\mu}, \quad C \gamma^{5}=\gamma^{5} C, \quad C^{\mathrm{T}}=C^{-1}=-C .
$$
}


(notice some reordering of the symbols $\imath_{n}$ as compared to (C.4)!) where the integrands of the integrals

$$
\begin{aligned}
\mathbb{X}_{\imath_{1} \imath_{2} \imath_{3} \imath_{4}}^{I}=\frac{i}{2} \operatorname{tr} \int \frac{\mathrm{d}^{4} k}{(2 \pi)^{4}} i \mathscr{S}(k) \gamma^{\mu_{2}} t_{\alpha_{2}} \mathscr{S}\left(k-l_{2}\right) \gamma^{\mu_{3}} t_{\alpha_{3}} \mathscr{S}\left(k-l_{2}-l_{3}\right) \gamma^{\mu_{4}}\left\{t_{\alpha_{4}}, t_{\alpha_{1}}\right\} \times \\
\times\left\{\tilde{g}\left(k+l_{1}\right)-\tilde{g}\left(k+l_{4}\right)\right\} \tilde{g}(k) \tilde{g}\left(k-l_{2}\right) \tilde{g}\left(k-l_{2}-l_{3}\right),
\end{aligned}
$$

and

$$
\begin{aligned}
\mathbb{X}_{l_{1} \imath_{2} \imath_{3} \imath_{4}}^{I I}=\frac{i}{2} e_{\alpha_{4} \alpha_{1}}^{\kappa} \operatorname{tr} \int \frac{\mathrm{d}^{4} k}{(2 \pi)^{4}} i \mathscr{S}(k) \gamma^{\mu_{2}} t_{\alpha_{2}} \mathscr{S}\left(k-l_{2}\right) \gamma^{\mu_{3}} t_{\alpha_{3}} \mathscr{S}\left(k-l_{2}-l_{3}\right) \gamma^{\mu_{4}} t_{\kappa} \times \\
\times\left\{\tilde{g}\left(k+l_{1}\right)+\tilde{g}\left(k+l_{4}\right)-2\right\} \tilde{g}(k) \tilde{g}\left(k-l_{2}\right) \tilde{g}\left(k-l_{2}-l_{3}\right) .
\end{aligned}
$$

vanish in the limit $\Lambda \rightarrow \infty$. (The term corresponding to -2 in the curly brackets of $\mathbb{X}^{I I}$ originates from the contribution of the $\langle A A A\rangle$ vertex to the considered ST identity.) The factors $\tilde{g}(k+l)$ can be now expanded in powers of the external momentum $l$. Performing next the integrals over the angular variables one finds that in the limit $\Lambda \rightarrow \infty$ the fermionic contribution can be written in the form

$$
\boldsymbol{\Omega}_{4 F} \equiv \boldsymbol{\Omega}_{4 F}^{\mathbb{A}}+\boldsymbol{\Omega}_{4 F}^{\mathbb{N}}=(\mathbb{A}+\mathbb{N})_{\imath_{1} \imath_{2} \imath_{3} \imath_{4}}+(\mathbb{A}+\mathbb{N})_{\imath_{1} \imath_{4} \imath_{3} \imath_{2}}+(\mathbb{A}+\mathbb{N})_{\imath_{1} \imath_{2} \imath_{4} \imath_{3}}+\mathcal{O}\left(\Lambda^{-1}\right)
$$

in which

$$
\begin{aligned}
\mathbb{A}_{\imath_{1} \imath_{2} \imath_{3} \imath_{4}}=\frac{\mathscr{C}_{0}}{(4 \pi)^{2}} \epsilon^{\nu \mu_{2} \mu_{3} \mu_{4}}\{ & \frac{1}{24}\left(l_{3}-l_{2}\right)_{\nu} e^{\kappa}{ }_{\alpha_{4} \alpha_{1}} \operatorname{tr}\left(\mathfrak{f}_{\kappa}\left\{\mathfrak{f}_{\alpha_{3}}, \mathfrak{f}_{\alpha_{2}}\right\}\right)+ \\
& \left.+\frac{1}{8}\left(l_{1}-l_{4}\right)_{\nu} e^{\kappa}{ }_{\alpha_{3} \alpha_{2}} \operatorname{tr}\left(\mathfrak{f}_{\kappa}\left\{\mathfrak{f}_{\alpha_{4}}, \mathfrak{f}_{\alpha_{1}}\right\}\right)\right\},
\end{aligned}
$$

is the true anomaly, while

$$
\begin{aligned}
& \mathbb{N}_{\underline{1}_{1} 2_{2} \imath_{3} \imath_{4}}= \\
& =\frac{i \mathscr{C}_{0}}{(4 \pi)^{2}} \times \frac{1}{24}\left\{\eta^{\nu \mu_{2}} \eta^{\mu_{3} \mu_{4}}+\eta^{\nu \mu_{3}} \eta^{\mu_{2} \mu_{4}}+\eta^{\nu \mu_{4}} \eta^{\mu_{2} \mu_{3}}\right\} \times \\
& \quad \times\left\{\frac{1}{3}\left(l_{3}-l_{2}\right)_{\nu} e^{\kappa}{ }_{\alpha_{4} \alpha_{1}} \operatorname{tr}\left(\mathfrak{f}_{\kappa}\left[\mathfrak{f}_{\alpha_{2}}, \mathfrak{f}_{\alpha_{3}}\right]\right)+\left(l_{4}-l_{1}\right)_{\nu} \operatorname{tr}\left(\left\{\mathfrak{f}_{\alpha_{2}}, \mathfrak{f}_{\alpha_{3}}\right\}\left\{\mathfrak{f}_{\alpha_{4}}, \mathfrak{f}_{\alpha_{1}}\right\}\right)\right\}+ \\
& +\frac{i \mathscr{C}_{1}}{(4 \pi)^{2}} \times \frac{2}{3}\left\{-\left(l_{2}+2 l_{3}\right)^{\mu_{2}} \eta^{\mu_{3} \mu_{4}}+\left(2 l_{2}+l_{3}\right)^{\mu_{3}} \eta^{\mu_{2} \mu_{4}}-\left(l_{2}-l_{3}\right)^{\mu_{4}} \eta^{\mu_{2} \mu_{3}}\right\} \times \\
& \quad \times e_{\alpha_{4} \alpha_{1}}^{\kappa} \operatorname{tr}\left(\mathfrak{f}_{\kappa}\left[\mathfrak{f}_{\alpha_{2}}, \mathfrak{f}_{\alpha_{3}}\right]\right) .
\end{aligned}
$$

is the cohomologically trivial breaking (the "spurious anomaly") of the ST identity. Thus, the complete result for $\boldsymbol{\Omega}_{4 F}$ depends (in the $\Lambda \rightarrow \infty$ limit) on only two integrals:

$$
\mathscr{C}_{0}=4 \int_{-\infty}^{0} \mathrm{~d} t g(t)^{3} g^{\prime}(t)=1, \quad \text { and } \quad \mathscr{C}_{1}=\int_{-\infty}^{0} \frac{\mathrm{d} t}{t}\left\{g(t)^{4}-g(t)^{3}\right\}
$$

of which only $\mathscr{C}_{1}$, entering the spurious part of the anomaly depends on the shape of the regularizing function $g\left(\mathscr{C}_{1}=\ln (4 / 3)\right.$ for $g(x)=\exp (x / 2)$, which corresponds to the 
prescription (3.1)), while $\mathscr{C}_{0}$, which multiplies the true anomaly, is independent of the specific shape of $g$ and depends only on the boundary conditions (C.2).

To close the analysis of the anomalies we will argue that in the regularization (3.1) the ST identity involving the $\langle A A A A A\rangle$ vertex is free of anomaly, that is that $\boldsymbol{\Omega}_{5 F}$ vanishes in the limit $\Lambda \rightarrow \infty$. To this end we first notice that all integrals that enter in this identity, whose form is analogous to (5.38), are (at worst) logarithmically divergent. As far as fermionic contributions are concerned relevant are only the $\left\langle A^{5}\right\rangle,\left\langle A^{4} \phi\right\rangle$ and $\left\langle A^{4}\right\rangle$ functions. In the logarithmically divergent contribution (C.4) to the $\left\langle A^{4}\right\rangle$ function one can make the replacement

$$
\mathscr{S}_{\Lambda}\left(p_{i}\right) \mapsto \tilde{g}\left(p_{i}\right) \mathscr{S}\left(p_{i}\right)
$$

because the terms of the integral (C.4) omitted in this way all vanish in the limit $\Lambda \rightarrow \infty$. In the convergent fermionic diagrams contributing to the $\left\langle A^{5}\right\rangle$ and $\left\langle A^{4} \phi\right\rangle$ functions $\Lambda$ can be sent to infinity. However, to make the cancellations in the ST identity manifest, the fermionic contribution to $\left\langle A^{5}\right\rangle$ (contracted with the momentum $l_{1}^{\mu_{1}}$ ) has to be decomposed using (C.9) into a combinations of integrals which individually are logarithmically divergent. Therefore, before making the decomposition (C.9), we multiply the unregulated integrands (i.e. the ones in which $\Lambda$ has been sent to infinity) of the fermionic contributions to the $\left\langle A^{5}\right\rangle$ and $\left\langle A^{4} \phi\right\rangle$ functions by the factor $\tilde{g}(k)^{4}$, where $k$ is the loop momentum. (Making instead the replacement (C.14) in the regulated integrands would produce five factors of $\tilde{g}$.) Performing next, just as before, appropriate shifts of the integration momentum gives $\boldsymbol{\Omega}_{5 F}$ in the form of the integral whose integrand is a homogeneous function of fourth degree in $\tilde{g}$ and vanishes in the limit $\Lambda \rightarrow \infty$. The integral is therefore similar to $\mathbb{X}^{I}$ given above. However, because the present integral is (unlike $\mathbb{X}^{I}$ ) only logarithmically divergent when all the $\tilde{g}$ factors are omitted, the momenta $q_{i}$ in $\tilde{g}\left(k+q_{i}\right)$ can give only a contribution of the order of $\Lambda^{-2} \times \mathcal{O}(\Lambda \ln \Lambda)$ which vanishes in the infinite cutoff limit. Thus, $\Omega_{5 F}$ indeed vanishes for $\Lambda \rightarrow \infty$. To complete the argument, it is sufficient to notice that the counterterm (5.40) does not break the considered ST identity either, because $\delta^{b} \mathrm{q}$ is an invariant tensor of the Lie algebra.

\section{RGE}

Here we show that eq. (7.5) indeed follows from (7.4). To this end we notice that the functional $\bar{r}_{n}^{\Lambda}$ defined by (7.6) and (7.3) is local and of renormalizable form when the regularization is neglected. The arguments of section 3 then ensure that Feynman diagrams generated by the auxiliary action ( $\Phi$ and $\mathcal{K}$ stand for all fields and antifields, respectively)

$$
\bar{I}_{n}^{\Lambda}[\Phi, \mathcal{K} ; g, c, \mu, \Lambda] \equiv I_{n}^{\Lambda}[\Phi, \mathcal{K} ; g, \mu, \Lambda]-c \bar{r}_{n}^{\Lambda}[\Phi, \mathcal{K} ; g, \mu, \Lambda]
$$

with the additional "coupling constant" $c$, are convergent. The RGE (7.6) can be rewritten as

$$
\left.\bar{R}_{n} \bar{I}_{n}^{\Lambda}[\Phi, \mathcal{K} ; g, c, \mu, \Lambda]\right|_{c=0}=0, \quad \text { with } \quad \bar{R}_{n} \equiv R_{n}+\frac{\partial}{\partial c}
$$


In the following it is convenient to decompose the $R_{n}$ operator defined in (7.3) (and analogously $\bar{R}_{n}$ ) in the following way

$$
R_{n}=B_{n}-\left(\gamma_{n}^{\Phi}\right)_{j}^{i} \Phi^{j} \cdot \frac{\delta}{\delta \Phi^{i}}
$$

$B_{n}$ contains then only derivatives w.r.t. the parameters (including the antifields):

$$
B_{n}=\mu \frac{\partial}{\partial \mu}+\beta_{n}^{A} \frac{\partial}{\partial g^{A}}-\left(\gamma_{n}^{\mathcal{K}}\right)_{i}^{j} \mathcal{K}_{j} \cdot \frac{\delta}{\delta \mathcal{K}_{i}} .
$$

Using eq. (D.2) and the functional integration by parts one easily checks that the generating functional

$$
\bar{Z}_{n}[J, \mathcal{K} ; g, c, \mu, \Lambda]=\int[\mathcal{D} \Phi] e^{i\left(\bar{I}_{n}^{\Lambda}+J_{k} \cdot \Phi^{k}\right)},
$$

satisfies the following RGE:

$$
\left.\overline{\mathscr{R}}_{n} \bar{Z}_{n}[J, \mathcal{K} ; g, c, \mu, \Lambda]\right|_{c=0}=\Theta \times \bar{Z}_{n}[J, \mathcal{K} ; g, 0, \mu, \Lambda]
$$

in which

$$
\overline{\mathscr{R}}_{n} \equiv \bar{B}_{n}+\left(\gamma_{n}^{\Phi}\right)^{i}{ }_{j} J_{i} \cdot \frac{\delta}{\delta J_{j}}=B_{n}+\frac{\partial}{\partial c}+\left(\gamma_{n}^{\Phi}\right)^{i}{ }_{j} J_{i} \cdot \frac{\delta}{\delta J_{j}} .
$$

Although $\Theta$ is a badly divergent factor

$$
\Theta=\sum_{i}\left(\mp \gamma_{n}^{\Phi}\right)_{i}^{i} \times \int \mathrm{d}^{4} x \delta_{\text {position }}^{(4)}(0)
$$

(upper/lower sign corresponds to bosonic/fermionic $\Phi^{i}$ ), it drops out from the RGE satisfied by the functional $\bar{W}_{n}$ generating connected Green's functions defined by

$$
e^{i \bar{W}_{n}[J, \mathcal{K} ; g, c, \mu, \Lambda]}=\frac{\bar{Z}_{n}[J, \mathcal{K} ; g, c, \mu, \Lambda]}{\bar{Z}_{n}[0, \mathcal{K} ; g, c, \mu, \Lambda]}
$$

which satisfies the simple relation

$$
\left.\overline{\mathscr{R}}_{n} \bar{W}_{n}[J, \mathcal{K} ; g, c, \mu, \Lambda]\right|_{c=0}=0 .
$$

Passing next to the functional $\bar{\Gamma}_{n}^{\Lambda} \equiv \Gamma\left[\bar{I}_{n}^{\Lambda}\right]$ generating 1PI functions, which is given as usually by the Legendre transform

$$
\bar{\Gamma}_{n}^{\Lambda}[\Phi, \mathcal{K} ; g, c, \mu, \Lambda] \equiv \bar{W}_{n}\left[\mathcal{J}^{\Phi}, \mathcal{K} ; g, c, \mu, \Lambda\right]-\mathcal{J}_{k}^{\Phi} \cdot \Phi^{k},
$$

with the source $\mathcal{J}^{\Phi}$ determined by the condition

$$
\left.\frac{\delta \bar{W}_{n}[J, \mathcal{K} ; g, c, \mu, \Lambda]}{\delta J_{i}(x)}\right|_{J=\mathcal{J}^{\Phi}}=\Phi^{i}(x),
$$

and using the inverse relations

$$
\frac{\delta \bar{\Gamma}_{n}^{\Lambda}[\Phi, \mathcal{K} ; g, c, \mu, \Lambda]}{\delta \Phi^{i}(x)}=\mp \mathcal{J}_{i}^{\Phi}(x),
$$


one finds that

$$
\begin{aligned}
\bar{R}_{n} \bar{\Gamma}_{n}^{\Lambda}[\Phi, \mathcal{K} ; g, c, \mu, \Lambda] & =\left.\left\{\bar{B}_{n} \bar{W}_{n}[J, \mathcal{K} ; g, c, \mu, \Lambda]+\left(\gamma_{n}^{\Phi}\right)_{j}^{i}{ }_{j} J_{i} \cdot \frac{\delta \bar{W}_{n}[J, \mathcal{K} ; g, c, \mu, \Lambda]}{\delta J_{j}}\right\}\right|_{J=\mathcal{J}^{\Phi}} \\
& =\left.\overline{\mathscr{R}}_{n} \bar{W}_{n}[J, \mathcal{K} ; g, c, \mu, \Lambda]\right|_{J=\mathcal{J}^{\Phi}} .
\end{aligned}
$$

This means that

$$
\left.\bar{R}_{n} \bar{\Gamma}_{n}^{\Lambda}[\Phi, \mathcal{K} ; g, c, \mu, \Lambda]\right|_{c=0}=0 .
$$

Since $\Gamma_{n}^{\Lambda} \equiv \Gamma\left[I_{n}^{\Lambda}\right]=\bar{\Gamma}_{n}^{\Lambda}[\Phi, \mathcal{K} ; g, 0, \mu, \Lambda],(\mathrm{D} .15)$ implies that

$$
R_{n} \Gamma_{n}^{\Lambda}[\Phi, \mathcal{K} ; g, \mu, \Lambda]=-\left.\frac{\partial \bar{\Gamma}_{n}^{\Lambda}[\Phi, \mathcal{K} ; g, c, \mu, \Lambda]}{\partial c}\right|_{c=0},
$$

However, from (D.1) it follows immediately that

$$
\left.\frac{\partial \bar{\Gamma}_{n}^{\Lambda}[\Phi, \mathcal{K} ; g, c, \mu, \Lambda]}{\partial c}\right|_{c=0}=-\bar{r}_{n}^{\Lambda}+\mathcal{O}\left(\hbar^{n+2}\right) .
$$

To see that the quantum correction in (D.17) is indeed of order $\mathcal{O}\left(\hbar^{n+2}\right)$, it is sufficient to notice that $c$-dependent contributions to $\bar{\Gamma}_{n}^{\Lambda}$ (except for the tree-level one, i.e. $-c \bar{r}_{n}^{\Lambda}$ ) are generated only by loop diagrams which contain one or more $-c \bar{r}_{n}^{\Lambda}$ vertices. Since by the inductive hypothesis $\bar{r}_{n}^{\Lambda}=\mathcal{O}\left(\hbar^{n+1}\right)$, such diagrams are necessarily at least of order of $\mathcal{O}\left(\hbar^{n+2}\right)$. Finally, using (7.7) and the $\Lambda$-independence of the coefficients in $R_{n}$ one concludes that eq. (7.5) indeed follows from (7.4).

\section{E DimReg- $\overline{M S}$ beta function $\check{\boldsymbol{\beta}}$}

In this appendix, for the reader's convenience, we recall in our notation (see section 2) the Jack-Osborn [48] expressions for the two-loop contributions to the beta functions $\check{\beta}$ in the DimReg- $\overline{\mathrm{MS}}$ scheme. We begin, however, with Machacek-Vaughn [49-51] formulae for two-loop contributions to the anomalous dimensions $\check{\gamma}$ of the scalars and left-chiral Weyl fermions in this scheme in the Landau gauge:

$$
\begin{aligned}
\check{\gamma}_{(2) i j}^{\phi}= & \left\{-\frac{35}{3} \operatorname{tr}\left[e_{\alpha} e_{\beta}\right]+\frac{11}{12} \operatorname{tr}\left[\mathcal{T}_{\alpha} \mathcal{T}_{\beta}\right]+\frac{20}{12} \operatorname{tr}\left[\mathfrak{f}_{\alpha} \mathfrak{f}_{\beta}\right]\right\}\left(\mathcal{T}^{\alpha} \mathcal{T}^{\beta}\right)_{i j}+ \\
& +\frac{1}{12} \lambda_{i k l n} \lambda_{j}{ }^{k l n}+\frac{3}{2}\left[\left(\mathcal{T}^{\alpha} \mathcal{T}_{\alpha}\right)^{2}\right]_{i j}-\frac{5}{2} \operatorname{tr}\left\{\mathfrak{f}^{\alpha} \mathfrak{f}_{\alpha} Y_{i}^{*} Y_{j}+c c .\right\}+ \\
& -\frac{3}{4} \operatorname{tr}\left\{Y_{i} Y_{j}^{*} Y^{\ell} Y_{\ell}^{*}+c c .\right\}-\frac{1}{2} \operatorname{tr}\left\{Y_{i}^{*} Y^{\ell} Y_{j}^{*} Y_{\ell}+c c .\right\} \\
\check{\gamma}_{(2)}^{F}= & \left\{\frac{25}{4} \operatorname{tr}\left[e_{\alpha} e_{\beta}\right]-\frac{1}{4} \operatorname{tr}\left[\mathcal{T}_{\alpha} \mathcal{T}_{\beta}\right]-\operatorname{tr}\left[\mathfrak{f}_{\alpha} \mathfrak{f}_{\beta}\right]\right\} \mathfrak{f}^{\alpha} \mathfrak{f}^{\beta}-\frac{3}{2}\left(\mathfrak{f}_{\kappa} \mathfrak{f}^{\kappa}\right)^{2}-\frac{3}{8} \mathbb{Y}^{i j} Y_{j}^{*} Y_{i}+ \\
& -\frac{1}{8} Y_{i}^{*} Y^{j} Y_{j}^{*} Y^{i}-\frac{9}{2}\left[\mathcal{T}^{\alpha} \mathcal{T}_{\alpha}\right]^{i j} Y_{i}^{*} Y_{j}+\frac{7}{4} \mathfrak{f}_{\alpha} \mathfrak{f}^{\alpha} Y_{\ell}^{*} Y^{\ell}+\frac{1}{4} Y_{\ell}^{*} \mathfrak{f}_{\alpha}^{*} \mathfrak{f}^{\alpha *} Y^{\ell} .
\end{aligned}
$$

We have not found in the literature the analogous Landau gauge two-loop contributions to the anomalous dimensions of the vector fields. (Of course, anomalous dimensions of Abelian 
gauge fields can be read off from the expression for the beta function of the corresponding Abelian gauge couplings given below.)

Two-loop contributions to the beta functions of the parameters of the scalar fields potential can be extracted by taking the appropriate derivatives with respect to $\varphi$ (to save the space we write $\mathcal{M}_{X}$ for $\mathcal{M}_{X}(\varphi)$, and $\mathcal{M}_{X}^{4}$ for $\left.\left[\mathcal{M}_{X}^{2}(\varphi)\right]^{2}\right)$ of

$$
\begin{aligned}
& \check{\beta}_{(2)}^{\mathcal{V}}(\varphi)=\left[\check{\gamma}_{(2)}^{\phi}(g)-\frac{1}{4} \operatorname{tr}\left[e_{\alpha} e_{\beta}\right] \mathcal{T}^{\alpha} \mathcal{T}^{\beta}\right]_{j}^{i} \varphi^{j} \frac{\partial \mathcal{V}(\varphi)}{\partial \varphi^{i}}+\varphi^{\mathrm{T}} \mathcal{T}^{\alpha} \mathcal{T}^{\beta} \mathcal{M}_{S}^{2} \mathcal{T}_{\beta} \mathcal{T}_{\alpha} \varphi+ \\
& +\left\{-\frac{161}{12} \operatorname{tr}\left[e_{\alpha} e_{\beta}\right]+\frac{7}{6} \operatorname{tr}\left[\mathcal{T}_{\alpha} \mathcal{T}_{\beta}\right]+\frac{8}{3} \operatorname{tr}\left[\mathfrak{f}_{\alpha} \mathfrak{f}_{\beta}\right]\right\}\left[\mathcal{M}_{V}^{4}\right]^{\alpha \beta}-\frac{27}{2} \operatorname{tr}\left\{e_{\alpha} \mathcal{M}_{V}^{2} e^{\alpha} \mathcal{M}_{V}^{2}\right\}+ \\
& -15\left[\mathcal{M}_{V}^{2}\right]_{\alpha \beta} \varphi^{\mathrm{T}} \mathcal{T}^{\alpha} \mathcal{T}^{\beta} \mathcal{T}^{\kappa} \mathcal{T}_{\kappa} \varphi-5\left[\mathcal{M}_{V}^{2}\right]_{\alpha \beta} \operatorname{tr}\left\{\mathcal{M}_{S}^{2} \mathcal{T}^{\alpha} \mathcal{T}^{\beta}\right\}-\operatorname{tr}\left\{\mathcal{M}_{S}^{4} \mathcal{T}^{\alpha} \mathcal{T}_{\alpha}\right\}+ \\
& -3 \operatorname{tr}\left\{\mathcal{M}_{S}^{2} \mathcal{T}^{\alpha} \mathcal{M}_{S}^{2} \mathcal{T}_{\alpha}\right\}-\frac{1}{2}\left[\mathcal{M}_{S}^{2}\right]^{i j} \delta^{k m} \delta^{l n} \mathcal{V}_{i k l}^{\prime \prime \prime}(\varphi) \mathcal{V}_{j m n}^{\prime \prime \prime}(\varphi)+ \\
& +6 \mathcal{M}_{V \alpha \beta}^{2} \operatorname{tr}\left\{\mathfrak{f}^{\alpha} \mathcal{M}_{F}^{*} \mathfrak{f}^{\beta *} \mathcal{M}_{F}+c c .\right\}+2 \operatorname{tr}\left\{\mathfrak{f}^{\alpha *} \mathfrak{f}_{\alpha}^{*}\left(\mathcal{M}_{F} \mathcal{M}_{F}^{*}\right)^{2}+c c .\right\}+ \\
& -\left[\mathcal{T}^{\alpha} \mathcal{T}_{\alpha} \varphi\right]^{j} \operatorname{tr}\left\{Y_{j} \mathcal{M}_{F}^{*} \mathcal{M}_{F} \mathcal{M}_{F}^{*}+c c .\right\}+\mathcal{M}_{S i j}^{2} \operatorname{tr}\left\{Y^{i} \mathcal{M}_{F}^{*} Y^{j} \mathcal{M}_{F}^{*}+c c .\right\}+ \\
& -\frac{1}{2} \mathbb{Y}^{i j}\left\{\mathcal{M}_{S}^{4}-\left[\mathcal{M}_{V}^{2}\right]_{\alpha \beta} \mathcal{T}^{\alpha} \varphi \varphi^{\mathrm{T}} \mathcal{T}^{\alpha}\right\}_{i j}+\operatorname{tr}\left\{Y^{j} Y_{j}^{*}\left(\mathcal{M}_{F} \mathcal{M}_{F}^{*}\right)^{2}+c c .\right\}+ \\
& +2 \operatorname{tr}\left\{Y^{j} \mathcal{M}_{F}^{*} Y_{j} \mathcal{M}_{F}^{*} \mathcal{M}_{F} \mathcal{M}_{F}^{*}+c c .\right\}+\operatorname{tr}\left\{Y^{j} \mathcal{M}_{F}^{*} \mathcal{M}_{F} Y_{j}^{*} \mathcal{M}_{F} \mathcal{M}_{F}^{*}+c c .\right\}
\end{aligned}
$$

where $\mathbb{Y}$ defined in (7.41). The above formula is basically the sum of the expressions (3.46) and (3.47) given in [48]. We have however explicitly rewritten the traces over Dirac's (or Majorana's) indices to the traces over the Weyl's indices, expressing (E.2) (and the formulae below) in terms of simpler matrices corresponding to the Weyl fermions. The second term in the bracket in the first line "correcting" the anomalous dimension originates from the fact that the contribution to $\breve{\beta}_{(2)}^{\mathcal{V}}(\varphi)$ naturally generated by the Feynman rules has the form

$$
\frac{1}{4} \varphi^{\mathrm{T}}\left\{\mathcal{T}^{\alpha}, \mathcal{T}^{\beta}\right\} \mathcal{M}_{S}^{2}\left\{\mathcal{T}_{\alpha}, \mathcal{T}_{\beta}\right\} \varphi=\varphi^{\mathrm{T}} \mathcal{T}^{\alpha} \mathcal{T}^{\beta} \mathcal{M}_{S}^{2} \mathcal{T}_{\beta} \mathcal{T}_{\alpha} \varphi-\frac{1}{4} \operatorname{tr}\left[e_{\alpha} e_{\beta}\right]\left[\mathcal{T}^{\alpha} \mathcal{T}^{\beta} \varphi\right]^{i} \mathcal{V}_{i}^{\prime}(\varphi)
$$

(the decomposition follows from the gauge invariance of the tree-level potential $\mathcal{V}$ ).

The beta function for the $\mathcal{M}_{F}(\varphi)$ matrix reads [48]

$$
\begin{aligned}
& \check{\beta}_{(2)}^{\mathcal{M}_{F}}(\varphi)=\left\{-\frac{97}{6} \operatorname{tr}\left[e_{\alpha} e_{\beta}\right]+\frac{11}{12} \operatorname{tr}\left[\mathcal{T}_{\alpha} \mathcal{T}_{\beta}\right]+\frac{5}{3} \operatorname{tr}\left[\mathfrak{f}_{\alpha} \mathfrak{f}_{\beta}\right]\right\}\left\{\mathcal{M}_{F} \mathfrak{f}^{\alpha} \mathfrak{f}^{\beta}+t p .\right\}+ \\
& -\frac{3}{2}\left\{\mathcal{M}_{F}\left(\mathfrak{f}_{\kappa} \mathfrak{f}^{\kappa}\right)^{2}+t p .\right\}+6\left[\mathcal{T}^{\alpha} \mathcal{T}_{\alpha} \varphi\right]^{i}\left\{Y_{i} \mathfrak{f}_{\kappa} \mathfrak{f}^{\kappa}+t p .\right\}-3\left\{Y_{\ell} \mathcal{M}_{F}^{*} Y^{\ell} \mathfrak{f}_{\alpha} \mathfrak{f}^{\alpha}+t p .\right\}+ \\
& -5\left\{Y^{\ell} \mathfrak{f}_{\alpha} \mathfrak{f}^{\alpha} \mathcal{M}_{F}^{*} Y_{\ell}+t p .\right\}-3\left\{Y^{\ell} Y_{\ell}^{*} \mathcal{M}_{F} \mathfrak{f}_{\alpha} \mathfrak{f}^{\alpha}+t p .\right\}+ \\
& +\frac{7}{4}\left\{Y^{\ell} Y_{\ell}^{*} \mathfrak{f}_{\alpha}^{*} f^{\alpha *} \mathcal{M}_{F}+t p .\right\}-\frac{11}{4}\left\{\mathcal{M}_{F} Y_{\ell}^{*} \mathfrak{f}_{\alpha}^{*} \mathfrak{f}^{\alpha *} Y^{\ell}+t p .\right\}+ \\
& -6\left[\mathcal{T}^{\alpha} \varphi\right]^{i} \mathcal{T}_{\alpha}^{j k}\left\{Y_{j} Y_{k}^{*} Y_{i}+t p .\right\}-\frac{3}{2}\left[\mathcal{T}^{\alpha} \mathcal{T}_{\alpha}\right]^{i j}\left\{\left[Y_{i} Y_{j}^{*} \mathcal{M}_{F}+t p .\right]+4 Y_{i} \mathcal{M}_{F}^{*} Y_{j}\right\}+ \\
& +3\left[\mathcal{T}^{\alpha} \mathcal{T}_{\alpha} \varphi\right]^{i}\left\{\left[Y^{j} Y_{j}^{*} Y_{i}+t p .\right]+4 Y^{j} Y_{i}^{*} Y_{j}\right\}+2 Y^{j} Y_{i}^{*} \mathcal{M}_{F} Y_{j}^{*} Y^{i}+ \\
& -2 Y^{i} Y^{j *} \mathcal{M}_{F} Y_{j}^{*} Y_{i}-\left\{Y^{j} \mathcal{M}_{F}^{*} Y^{\ell} Y_{\ell}^{*} Y_{j}+t p .\right\}-\frac{1}{8}\left\{Y^{i} Y_{j}^{*} Y^{j} Y_{i}^{*} \mathcal{M}_{F}+t p .\right\}+
\end{aligned}
$$




$$
\begin{aligned}
-2 \mathcal{V}_{i j k}^{\prime \prime \prime}(\varphi) Y^{i} Y^{j *} Y^{k}-\mathbb{Y}^{i j}\left\{Y_{i} \mathcal{M}_{F}^{*} Y_{j}+\frac{3}{8}\left[Y_{i} Y_{j}^{*} \mathcal{M}_{F}+t p .\right]\right\}+ \\
+Y^{i} \varphi^{j}\left\{\left[\frac{49}{4} \operatorname{tr}\left[e_{\alpha} e_{\beta}\right]-\frac{1}{4} \operatorname{tr}\left[\mathcal{T}_{\alpha} \mathcal{T}_{\beta}\right]-\operatorname{tr}\left[\mathfrak{f}_{\alpha} \mathfrak{f}_{\beta}\right]\right]\left[\mathcal{T}^{\alpha} \mathcal{T}^{\beta}\right]_{i j}-\frac{21}{2}\left[\left(\mathcal{T}^{\alpha} \mathcal{T}_{\alpha}\right)^{2}\right]_{i j}+\right. \\
\quad+\frac{1}{12} \lambda_{i k l n} \lambda_{j}{ }^{k l n}-\frac{5}{2} \operatorname{tr}\left\{\mathfrak{f}^{\alpha} \mathfrak{f}_{\alpha} Y_{i}^{*} Y_{j}+c c .\right\}-\frac{3}{4} \operatorname{tr}\left\{Y_{i} Y_{j}^{*} Y^{\ell} Y_{\ell}^{*}+c c .\right\}+ \\
\left.\quad-\frac{1}{2} \operatorname{tr}\left\{Y_{i}^{*} Y^{\ell} Y_{j}^{*} Y_{\ell}+c c .\right\}\right\} .
\end{aligned}
$$

Finally, those of the gauge couplings read [48-51]

$$
\begin{aligned}
\check{\beta}_{(2)}^{T_{\alpha}}=T_{\kappa}\{ & -\frac{1}{3}\left\{34 \operatorname{tr}\left[e^{\kappa} e^{\beta}\right]-\operatorname{tr}\left[\mathcal{T}^{\kappa} \mathcal{T}^{\beta}\right]-10 \operatorname{tr}\left[\mathfrak{f}^{\kappa} \mathfrak{f}^{\beta}\right]\right\} \operatorname{tr}\left[e_{\beta} e_{\alpha}\right]+ \\
& \left.+2 \operatorname{tr}\left[\mathcal{T}^{\kappa} \mathcal{T}_{\alpha} \mathcal{T}^{\beta} \mathcal{T}_{\beta}\right]+\operatorname{tr}\left[\mathfrak{f}^{\kappa} \mathfrak{f}_{\alpha} \mathfrak{f}^{\beta} \mathfrak{f}_{\beta}+c c .\right]+\frac{1}{2} \operatorname{tr}\left[\mathfrak{f}^{\kappa} \mathfrak{f}_{\alpha} Y_{i}^{*} Y^{i}+c c .\right]\right\} .
\end{aligned}
$$

\section{F The Standard Model case}

In this appendix we list the Standard Model two-loop $\beta$ functions in the $\Lambda$ - $\overline{\mathrm{MS}}$ scheme, the two-loop coefficient of quadratic divergence of the scalar fields and the factors $\theta$ relating renormalized parameters in this scheme to the ones in the DimReg- $\overline{\mathrm{MS}}$ scheme. We use the notation in which the scalar potential has the form

$$
\mathcal{V}(H)=m_{H}^{2} H^{\dagger} H+\lambda_{1}\left(H^{\dagger} H\right)^{2} .
$$

The normalization of the $H$ field VEV is such that $\left\langle H_{i}\right\rangle=\frac{1}{\sqrt{2}} v_{H} \delta_{i 2}$. The tree-level masses of the (usually most relevant) heavy states read

$$
M_{t}=\frac{1}{\sqrt{2}} y_{t} v_{H}, \quad M_{W}=\frac{1}{2} g_{w} v_{H}, \quad M_{Z}=\frac{1}{2} \sqrt{g_{w}^{2}+g_{y}^{2}} v_{H} .
$$

The strong coupling constant $g_{s}$ is normalized so that the adjoint representation generators $e_{\alpha_{c}}$ of the $\mathrm{SU}(3)_{C}$ group satisfy the relation

$$
\operatorname{tr}\left(e_{\alpha_{c}} e_{\beta_{c}}\right)=-3 g_{s}^{2} \delta_{\alpha_{c} \beta_{c}}
$$

All the Yukawa couplings other than $y_{t}$ are neglected.

The beta functions of the gauge couplings are the same in both schemes and read (see the explanation below (7.38))

$$
\begin{array}{ll}
\beta_{(1)}^{g_{w}}=-\frac{19}{6} g_{w}^{3}, & \beta_{(2)}^{g_{w}}=\frac{1}{6} g_{w}^{3}\left(72 g_{s}^{2}+35 g_{w}^{2}+9 g_{y}^{2}-9 y_{t}^{2}\right), \\
\beta_{(1)}^{g_{y}}=\frac{41}{6} g_{y}^{3}, & \beta_{(2)}^{g_{y}}=\frac{1}{18} g_{y}^{3}\left(264 g_{s}^{2}+81 g_{w}^{2}+199 g_{y}^{2}-51 y_{t}^{2}\right), \\
\beta_{(1)}^{g_{s}}=-7 g_{s}^{3}, & \beta_{(2)}^{g_{s}}=-\frac{1}{6} g_{s}^{3}\left(156 g_{s}^{2}-27 g_{w}^{2}-11 g_{y}^{2}+12 y_{t}^{2}\right) .
\end{array}
$$


The one- and two-loop pieces of the beta functions of the $\Lambda$ - $\overline{\mathrm{MS}}$ scheme coupling $\lambda_{1}$ read

$$
\begin{aligned}
\beta_{(1)}^{\lambda_{1}}= & 24 \lambda_{1}^{2}-3 \lambda_{1}\left(3 g_{w}^{2}+g_{y}^{2}-4 y_{t}^{2}\right)+\frac{9}{8} g_{w}^{4}+\frac{3}{4} g_{w}^{2} g_{y}^{2}+\frac{3}{8} g_{y}^{4}-6 y_{t}^{4} \\
\beta_{(2)}^{\lambda_{1}}= & -312 \lambda_{1}^{3}+\lambda_{1}^{2}\left(3\left(12+c_{3}\right)\left(3 g_{w}^{2}+g_{y}^{2}\right)-36\left(4+c_{5}\right) y_{t}^{2}\right)+ \\
& +\lambda_{1}\left\{\frac{1}{8}\left(\frac{3}{2} c_{15}-73\right) g_{w}^{4}+\left(\frac{39}{4}-36 c_{0}\right) g_{w}^{2} g_{y}^{2}+\right. \\
& +\frac{1}{24}\left(\frac{3}{2} c_{10}+629\right) g_{y}^{4}+\left(\frac{3}{2} c_{2}-3\right) y_{t}^{4}+ \\
& \left.+\left(8\left(10-c_{6}\right) g_{s}^{2}+\frac{9}{4}\left(10-c_{8}\right) g_{w}^{2}+\frac{1}{12}\left(170-c_{12}\right) g_{y}^{2}\right) y_{t}^{2}\right\}+ \\
& -\frac{1}{16}\left(9\left(4-c_{1}\right) g_{w}^{4}-6\left(28+c_{1}\right) g_{w}^{2} g_{y}^{2}+\left(76-3 c_{1}\right) g_{y}^{4}\right) y_{t}^{2}+ \\
+ & {\left[-8\left(4+12 c_{0}\right) g_{s}^{2}+27 c_{0} g_{w}^{2}+\left(c_{0}-\frac{8}{3}\right) g_{y}^{2}\right] y_{t}^{4}+\left(c_{4}+30\right) y_{t}^{6}+} \\
& +\frac{1}{192}\left\{3\left(1220+3 c_{9}\right) g_{w}^{6}-\left(1156+3 c_{16}\right) g_{w}^{4} g_{y}^{2}-\left(2236-3 c_{13}\right) g_{w}^{2} g_{y}^{4}+\right. \\
& \left.-\left(1516+3 c_{11}\right) g_{y}^{6}\right\}
\end{aligned}
$$

The one- and two-loop pieces of the beta function of the $\Lambda$ - $\overline{\mathrm{MS}}$ scheme mass parameter $m_{H}^{2}$ are

$$
\begin{aligned}
\beta_{(1)}^{m_{H}^{2}} / m_{H}^{2}= & 12 \lambda_{1}+6 y_{t}^{2}-\left(\frac{9}{2} g_{w}^{2}+\frac{3}{2} g_{y}^{2}\right) \\
\beta_{(2)}^{m_{H}^{2}} / m_{H}^{2}= & -60 \lambda_{1}^{2}+\left(24+\frac{3}{2} c_{3}\right) \lambda_{1}\left(3 g_{w}^{2}+g_{y}^{2}\right)+\left(\frac{3}{32} c_{14}-\frac{145}{16}\right) g_{w}^{4}+ \\
& +\left(\frac{15}{8}-9 c_{0}\right) g_{w}^{2} g_{y}^{2}+\left(\frac{c_{17}}{32}+\frac{557}{48}\right) g_{y}^{4}-\left(72+18 c_{5}\right) \lambda_{1} y_{t}^{2}+ \\
& +\frac{1}{24}\left[96\left(10-c_{6}\right) g_{s}^{2}+27\left(10-c_{8}\right) g_{w}^{2}+\left(170-c_{12}\right) g_{y}^{2}\right] y_{t}^{2}+\left[\frac{3}{4} c_{2}-\frac{27}{2}\right] y_{t}^{4} .
\end{aligned}
$$

The beta function of the $\Lambda$ - $\overline{\mathrm{MS}}$ scheme top quark Yukawa coupling reads

$$
\begin{aligned}
\beta_{(1)}^{y_{t}}=y_{t} & \left\{\frac{9}{2} y_{t}^{2}-8 g_{s}^{2}-\frac{9}{4} g_{w}^{2}-\frac{17}{12} g_{y}^{2}\right\} \\
\beta_{(2)}^{y_{t}}=y_{t} & \left\{y_{t}^{2}\left[\left(36-4 c_{7}\right) g_{s}^{2}+\frac{1}{32}\left(450-9 c_{21}\right) g_{w}^{2}+\left(\frac{131}{16}-\frac{c_{19}}{96}\right) g_{y}^{2}-12 \lambda_{1}\right]+\right. \\
& -12 y_{t}^{4}+\left(\frac{c_{18}}{3}-108\right) g_{s}^{4}+g_{s}^{2}\left(9 g_{w}^{2}+\frac{19}{9} g_{y}^{2}\right)+ \\
+ & {\left.\left[\left(\frac{c_{20}}{64}-\frac{23}{4}\right) g_{w}^{4}-\frac{3}{4} g_{w}^{2} g_{y}^{2}+\left(\frac{c_{22}}{16}+\frac{1187}{216}\right) g_{y}^{4}\right]+6 \lambda_{1}^{2}\right\} }
\end{aligned}
$$


The coefficients $c_{n}$ appearing in these beta functions read

$$
\begin{aligned}
& c_{0}=\frac{1}{3}, \quad c_{1}=1+12 \ln 2, \quad c_{2}=7+12 \ln \frac{3}{2}, \\
& c_{3}=2+3 \ln \frac{32}{9}, \quad c_{4}=3\left(1+6 \ln \frac{4}{3}\right), \quad c_{5}=3+4 \ln 2, \\
& c_{6}=5+12 \ln 2, \quad c_{7}=2+3 \ln \frac{16}{3}, \quad c_{8}=11+3 \ln \frac{9}{2},
\end{aligned}
$$

and

$$
\begin{aligned}
c_{9} & =257 \ln 2-9(19+26 \ln 3), & c_{10} & =145+82 \ln \frac{9}{2}, \\
c_{11} & =59+373 \ln 2-18 \ln 3, & c_{12} & =139+69 \ln 2+54 \ln 3, \\
c_{13} & =13-553 \ln 2+90 \ln 3, & c_{14} & =101-2(101 \ln 2-106 \ln 3), \\
c_{15} & =53-2(101 \ln 2-106 \ln 3), & c_{16} & =13 \ln 2+63(1+\ln 9), \\
c_{17} & =193+82 \ln \frac{9}{2}, & c_{18} & =103-348 \ln 2+216 \ln 3, \\
c_{19} & =298+411 \ln 2-42 \ln 3, & c_{20} & =295-6(101 \ln 2-106 \ln 3), \\
c_{21} & =26+3 \ln 18, & c_{22} & =\frac{13931}{108}+\frac{41}{18}(23 \ln 2+18 \ln 3) .
\end{aligned}
$$

Setting them to zero one recovers the beta functions of the DimReg- $\overline{\mathrm{MS}}$ scheme.

The two-loop coefficient in front of the quadratic divergence, normalized as in eqs. (7.18) and (9.1), has the form

$$
\begin{aligned}
f^{(2)}= & \frac{1}{2}\left(-3\left(\bar{c}_{3}-\bar{c}_{5}+6 \bar{c}_{6}+2 \bar{c}_{9}\right) g_{w}^{4}+6 \bar{c}_{5} g_{w}^{2} g_{y}^{2}-\left(\bar{c}_{3}-\bar{c}_{5}+10 \bar{c}_{6}\right) g_{y}^{4}\right)+ \\
& -\frac{1}{6}\left(96\left(\bar{c}_{7}+\bar{c}_{8}\right) g_{s}^{2}+27 \bar{c}_{7} g_{w}^{2}+\left(17 \bar{c}_{7}+8 \bar{c}_{8}\right) g_{y}^{2}\right) y_{t}^{2}+18 \bar{c}_{1} y_{t}^{4}+ \\
& +72 \bar{c}_{2} \lambda_{1} y_{t}^{2}+36 \lambda_{1}^{2} \ln \frac{4}{3}-3 \bar{c}_{4} \lambda_{1}\left(3 g_{w}^{2}+g_{y}^{2}\right),
\end{aligned}
$$

where

$$
\begin{aligned}
& \bar{c}_{1}=\frac{1}{12}(64 \ln 2+15 \ln 3-25 \ln 5-11 \ln 11) \\
& \bar{c}_{2}=\frac{1}{2}\left(-1+9 \ln 2-\frac{45}{2} \ln 3+\frac{25}{2} \ln 5\right) \\
& \bar{c}_{3}=\frac{17}{8}-\frac{51}{4} \ln 2+\frac{459}{16} \ln 3-\frac{125}{8} \ln 5 \\
& \bar{c}_{4}=\frac{29}{16}-\frac{1901}{72} \ln 2-\frac{369}{32} \ln 3+\frac{125}{72} \ln 5+\frac{847}{72} \ln 11, \\
& \bar{c}_{5}=-\frac{1}{8}+\frac{189}{32} \ln 2-\frac{45}{16} \ln 3 \\
& \bar{c}_{6}=-\frac{13}{4}+\frac{61}{2} \ln 2-54 \ln 3+25 \ln 5, \\
& \bar{c}_{7}=-\frac{13}{8}+\frac{667}{18} \ln 2+21 \ln 3+\frac{25}{9} \ln 5-\frac{187}{9} \ln 11,
\end{aligned}
$$




$$
\begin{aligned}
& \bar{c}_{8}=\frac{1}{2}\left(-\frac{9}{4}-21 \ln 2+9 \ln 3\right) \\
& \bar{c}_{9}=\frac{39}{4}-\frac{9695}{96} \ln 2+\frac{81}{4} \ln 3-\frac{625}{24} \ln 5+\frac{847}{24} \ln 11 .
\end{aligned}
$$

Finally, the factors $\theta$ appearing in the formula (6.3) relating the renormalized parameters of the $\Lambda$ - $\overline{\mathrm{MS}}$ and DimReg- $\overline{\mathrm{MS}}$ schemes read

$$
\begin{aligned}
\theta_{(1)}^{g_{s}}(g)= & {\left[\frac{29}{4} \ln 2-\frac{9}{2} \ln 3-\frac{47}{48}\right] g_{s}^{3}, } \\
\theta_{(1)}^{g_{w}}(g)= & {\left[\frac{49}{12} \ln 2-3 \ln 3-\frac{371}{288}\right] g_{w}^{3}, } \\
\theta_{(1)}^{g_{y}}(g)= & -\frac{1}{288}(887+984 \ln 2) g_{y}^{3}, \\
\theta_{(1)}^{\lambda_{1}}(g)= & -\frac{1}{8}\left(2 g_{w}^{2} g_{y}^{2}+3 g_{w}^{4}+g_{y}^{4}\right)+\frac{1}{8}\left[2+3 \ln \frac{32}{9}\right] \lambda_{1}\left(3 g_{w}^{2}+g_{y}^{2}\right)+ \\
& -\left[\frac{9}{2}+6 \ln 2\right] \lambda_{1} y_{t}^{2}, \\
\theta_{(1)}^{m_{H}^{2}}(g)= & m_{H}^{2}\left\{\frac{1}{16}\left[2+3 \ln \frac{32}{9}\right]\left(3 g_{w}^{2}+g_{y}^{2}\right)-\frac{3}{4}(3+4 \ln 2) y_{t}^{2}\right\} \\
\theta_{(1)}^{y_{t}}(g)= & y_{t}\left\{\frac{4}{3} g_{s}^{2}+\frac{3}{32}\left[3 \ln \frac{32}{9}-2\right] g_{w}^{2}+\left[\frac{7}{144}+\frac{3}{32} \ln \frac{32}{9}\right] g_{y}^{2}+\right. \\
& \left.\quad-\left[\frac{5}{4}+\frac{3}{4} \ln \frac{16}{3}\right] y_{t}^{2}\right\} .
\end{aligned}
$$

\section{G Basic 2-loop integrals}

Here we list the nine basic 2-loop integrals to which the genuine 2-loop vacuum graphs $A$ - $J$ shown in figure 1 (section 8) regularized using the prescription of section 3 can be reduced. We also calculate their divergent parts.

The integrals are

$$
\begin{gathered}
I_{\Lambda}^{\mathrm{tot}}\left(m_{1}, m_{2}, m_{3} \mid n_{1}, n_{2}, n_{3}\right) \equiv \\
=i^{2} \int \frac{\mathrm{d}^{4} k}{(2 \pi)^{4}} \int \frac{\mathrm{d}^{4} q}{(2 \pi)^{4}} \frac{e^{\left(n_{1}-1\right) \frac{k^{2}}{\Lambda^{2}}}}{\mathcal{R}(k)^{2}-m_{1}^{2}} \frac{e^{\left(n_{2}-1\right) \frac{q^{2}}{\Lambda^{2}}}}{\mathcal{R}(q)^{2}-m_{2}^{2}} \frac{e^{\left(n_{3}-1\right) \frac{(k+q)}{\Lambda^{2}}}}{\mathcal{R}(k+q)^{2}-m_{3}^{2}}, \\
J_{\Lambda}^{\mathrm{tot}}\left(m_{1}, m_{2} \mid n_{1}, n_{2}, n_{3}\right) \equiv \\
=i^{2} \int \frac{\mathrm{d}^{4} k}{(2 \pi)^{4}} \int \frac{\mathrm{d}^{4} q}{(2 \pi)^{4}} e^{n_{3} \frac{(k+q)^{2}}{\Lambda^{2}}} \frac{e^{\left(n_{1}-1\right) \frac{k^{2}}{\Lambda^{2}}}}{\mathcal{R}(k)^{2}-m_{1}^{2}} \frac{e^{\left(n_{2}-1\right) \frac{q^{2}}{\Lambda^{2}}}}{\mathcal{R}(q)^{2}-m_{2}^{2}}, \\
N_{2, \Lambda}^{\mathrm{tot}}\left(m_{1}, m_{2} \mid n_{1}, n_{2}, n_{3}\right) \equiv \\
=i^{2} \int \frac{\mathrm{d}^{4} k}{(2 \pi)^{4}} \int \frac{\mathrm{d}^{4} q}{(2 \pi)^{4}} e^{n_{3} \frac{(k+q)}{\Lambda^{2}}} \frac{e^{\left(n_{1}-1\right) \frac{k^{2}}{\Lambda^{2}}}}{\mathcal{R}(k)^{2}-m_{1}^{2}} \frac{e^{\left(n_{2}-1\right) \frac{q^{2}}{\Lambda^{2}}}}{\mathcal{R}(q)^{2}-m_{2}^{2}} \times \frac{(k \cdot q)^{2}}{k^{2} q^{2}}, \\
M_{1, \Lambda}^{\mathrm{tot}}\left(m_{1}, m_{3} \mid n_{1}, n_{2}, n_{3}\right) \equiv \\
=-i^{2} \int \frac{\mathrm{d}^{4} k}{(2 \pi)^{4}} \int \frac{\mathrm{d}^{4} q}{(2 \pi)^{4}} e^{n_{2} \frac{(k+q)^{2}}{\Lambda^{2}}} \frac{e^{\left(n_{1}-1\right) \frac{k^{2}}{\Lambda^{2}}}}{\mathcal{R}(k)^{2}-m_{1}^{2}} \frac{e^{\left(n_{3}-1\right) \frac{q^{2}}{\Lambda^{2}}}}{\mathcal{R}(q)^{2}-m_{3}^{2}} \times \frac{k \cdot q}{q^{2}},
\end{gathered}
$$




$$
\begin{aligned}
& I_{1, \Lambda}^{\mathrm{tot}}\left(m_{1}, m_{2}, m_{3} \mid n_{1}, n_{2}, n_{3}\right) \equiv \\
& =i^{2} \int \frac{\mathrm{d}^{4} k}{(2 \pi)^{4}} \int \frac{\mathrm{d}^{4} q}{(2 \pi)^{4}} \frac{e^{\left(n_{1}-1\right) \frac{k^{2}}{\Lambda^{2}}}}{\mathcal{R}(k)^{2}-m_{1}^{2}} \frac{e^{\left(n_{2}-1\right) \frac{q^{2}}{\Lambda^{2}}}}{\mathcal{R}(q)^{2}-m_{2}^{2}} \frac{e^{\left(n_{3}-1\right) \frac{(k+q)^{2}}{\Lambda^{2}}}}{\mathcal{R}(k+q)^{2}-m_{3}^{2}} \times \frac{1}{(k+q)^{2}}, \\
& I_{2, \Lambda}^{\text {tot }}\left(m_{1}, m_{2}, m_{3} \mid n_{1}, n_{2}, n_{3}\right) \equiv \\
& =i^{2} \int \frac{\mathrm{d}^{4} k}{(2 \pi)^{4}} \int \frac{\mathrm{d}^{4} q}{(2 \pi)^{4}} \frac{e^{\left(n_{1}-1\right) \frac{k^{2}}{\Lambda^{2}}}}{\mathcal{R}(k)^{2}-m_{1}^{2}} \frac{e^{\left(n_{2}-1\right) \frac{q^{2}}{\Lambda^{2}}}}{\mathcal{R}(q)^{2}-m_{2}^{2}} \frac{e^{\left(n_{3}-1\right) \frac{(k+q)}{\Lambda^{2}}}}{\mathcal{R}(k+q)^{2}-m_{3}^{2}} \times \frac{1}{(k+q)^{2} q^{2}}, \\
& J_{1, \Lambda}^{\text {tot }}\left(m_{1}, m_{2} \mid n_{1}, n_{2}, n_{3}\right) \equiv \\
& =i^{2} \int \frac{\mathrm{d}^{4} k}{(2 \pi)^{4}} \int \frac{\mathrm{d}^{4} q}{(2 \pi)^{4}} e^{n_{3} \frac{(k+q)^{2}}{\Lambda^{2}}} \frac{e^{\left(n_{1}-1\right) \frac{k^{2}}{\Lambda^{2}}}}{\mathcal{R}(k)^{2}-m_{1}^{2}} \frac{e^{\left(n_{2}-1\right) \frac{q^{2}}{\Lambda^{2}}}}{\mathcal{R}(q)^{2}-m_{2}^{2}} \frac{1}{k^{2}}, \\
& J_{2, \Lambda}^{\text {tot }}\left(m_{1}, m_{2} \mid n_{1}, n_{2}, n_{3}\right) \equiv \\
& =i^{2} \int \frac{\mathrm{d}^{4} k}{(2 \pi)^{4}} \int \frac{\mathrm{d}^{4} q}{(2 \pi)^{4}} e^{n_{3} \frac{(k+q)^{2}}{\Lambda^{2}}} \frac{e^{\left(n_{1}-1\right) \frac{k^{2}}{\Lambda^{2}}}}{\mathcal{R}(k)^{2}-m_{1}^{2}} \frac{e^{\left(n_{2}-1\right) \frac{q^{2}}{\Lambda^{2}}}}{\mathcal{R}(q)^{2}-m_{2}^{2}} \frac{1}{k^{2} q^{2}}, \\
& M_{2, \Lambda}^{\text {tot }}\left(m_{1}, m_{3} \mid n_{1}, n_{2}, n_{3}\right) \equiv \\
& =-i^{2} \int \frac{\mathrm{d}^{4} k}{(2 \pi)^{4}} \int \frac{\mathrm{d}^{4} q}{(2 \pi)^{4}} e^{n_{2} \frac{(k+q)}{\Lambda^{2}}} \frac{e^{\left(n_{1}-1\right) \frac{k^{2}}{\Lambda^{2}}}}{\mathcal{R}(k)^{2}-m_{1}^{2}} \frac{e^{\left(n_{3}-1\right) \frac{q^{2}}{\Lambda^{2}}}}{\mathcal{R}(q)^{2}-m_{3}^{2}} \times \frac{k \cdot q}{q^{2} k^{2}} .
\end{aligned}
$$

All these integrals are convergent provided all $n_{i}$ 's in the exponents are nonnegative and at least two of them are strictly positive. The integrals arising in the decompositions of the genuine 2-loop vacuum diagrams of figure 1 all fulfill these conditions.

Strictly speaking, only the integrals (G.1)-(G.4) are independent; the remaining ones are their linear combinations. For instance,

$$
\begin{aligned}
& I_{2, \Lambda}^{\mathrm{tot}}\left(m_{1}, m_{2}, m_{3} \mid n_{1}, n_{2}, n_{3}\right)=\frac{1}{m_{2}^{2} m_{3}^{2}}\left\{I_{\Lambda}^{\mathrm{tot}}\left(m_{1}, m_{2}, m_{3} \mid n_{1}, n_{2}-1, n_{3}-1\right)+\right. \\
& \quad-I_{\Lambda}^{\mathrm{tot}}\left(m_{1}, m_{2}, 0 \mid n_{1}, n_{2}-1, n_{3}-1\right)-I_{\Lambda}^{\mathrm{tot}}\left(m_{1}, 0, m_{3} \mid n_{1}, n_{2}-1, n_{3}-1\right)+ \\
& \left.\quad+I_{\Lambda}^{\mathrm{tot}}\left(m_{1}, 0,0 \mid n_{1}, n_{2}-1, n_{3}-1\right)\right\} .
\end{aligned}
$$

However, because the integrals on the right hand side of (G.10) have lower values of $n_{i}$ 's, they can in principle be divergent even if the one on the right hand side is not. In the case of the diagrams $A-J$ of figure 1 the integrals (G.1)-(G.4) arising on right hand sides of decompositions analogous to (G.10) have still nonnegative $n_{i}$ 's but in some cases more than one $n_{i}$ vanishes. The decompositions like (G.10) are then justified if one makes the replacement

$$
n_{i} \rightarrow n_{i}+c_{i} \varepsilon, \quad c_{i}>0, \varepsilon>0,
$$

first. Singularities arising for $\varepsilon \rightarrow 0$ cancel out in the sums like (G.10). Below we give explicit expressions only for the integrals (G.1)-(G.4).

It is convenient to start with the following auxiliary integral

$$
\mathbb{I}_{\Lambda} \equiv I_{\Lambda}\left(m_{1}, m_{2}, m_{3} \mid n_{1}, n_{2}, n_{3}\right) \equiv i^{2} \int \frac{\mathrm{d}^{4} k}{(2 \pi)^{4}} \int \frac{\mathrm{d}^{4} q}{(2 \pi)^{4}} \frac{e^{n_{1} \frac{k^{2}}{\Lambda^{2}}}}{k^{2}-m_{1}^{2}} \frac{e^{n_{2} \frac{q^{2}}{\Lambda^{2}}}}{q^{2}-m_{2}^{2}} \frac{e^{n_{3} \frac{(k+q)^{2}}{\Lambda^{2}}}}{(k+q)^{2}-m_{3}^{2}} .
$$


In the Schwinger parametrization $\mathbb{I}_{\Lambda}$ takes the form

$$
(4 \pi)^{4} \mathbb{I}_{\Lambda}=-\int_{\mathbb{R}_{+}^{3}} \mathrm{~d}^{3} \xi e^{-\xi_{1} m_{1}^{2}} e^{-\xi_{2} m_{2}^{2}} e^{-\xi_{3} m_{3}^{2}} H_{\Lambda}(\xi),
$$

where $\left(\epsilon_{i} \equiv n_{i} / \Lambda^{2}\right)$

$$
H_{\Lambda}(\xi)=\left[\left(\xi_{1}+\epsilon_{1}\right)\left(\xi_{2}+\epsilon_{2}\right)+\left(\xi_{1}+\epsilon_{1}\right)\left(\xi_{3}+\epsilon_{3}\right)+\left(\xi_{2}+\epsilon_{2}\right)\left(\xi_{3}+\epsilon_{3}\right)\right]^{-2} .
$$

With the help of the identity

$$
e_{1} e_{2} e_{3}=\left\{-2+\sum_{i} e_{i}\right\}+\sum_{i, j ; i<j}\left(1-e_{i}\right)\left(1-e_{j}\right)-\prod_{i}\left(1-e_{i}\right),
$$

$\mathbb{I}_{\Lambda}$ can be split into several pieces:

$$
\mathbb{I}_{\Lambda}=\mathbb{I}_{\Lambda}^{\text {div }}-\sum_{i, j ; i<j} \mathbb{I}_{\Lambda}^{(2) i j}+\mathbb{I}_{\Lambda}^{(3)}
$$

where

$$
(4 \pi)^{4} \mathbb{I}_{\Lambda}^{\mathrm{div}}=-\int_{\mathbb{R}_{+}^{3}} \mathrm{~d}^{3} \xi\left\{-2+\sum_{i=1}^{3} e^{-\xi_{i} m_{i}^{2}}\right\} H_{\Lambda}(\xi),
$$

etc. Using the inequalities:

$$
0 \leq H_{\Lambda}(\xi) \leq H_{\infty}(\xi)=\left[\xi_{1} \xi_{2}+\xi_{1} \xi_{3}+\xi_{2} \xi_{3}\right]^{-2}
$$

and $1-e^{-x} \leq 2 x /(1+x)$ (the latter valid for $\left.x \geq 0\right)$ it is easy to prove that the integrals $\mathbb{I}_{\Lambda}^{(2) i j}$ and $\mathbb{I}_{\Lambda}^{(3)}$ have finite limits for $\Lambda \rightarrow \infty$. Aiming at computing the divergent parts of the diagrams $A$ - $J$ in figure 1 we focus, therefore, on $\mathbb{I}_{\Lambda}^{\text {div }}$ only.

Two out of the three integrals over $\xi_{i}$ in $\mathbb{I}_{\Lambda}^{\text {div }}$ are elementary. Taking them we get

$$
\begin{aligned}
(4 \pi)^{4} \mathbb{I}_{\Lambda}^{\text {div }}= & -\mathcal{K}_{\Lambda}\left(m_{1} \mid n_{1}, n_{2}, n_{3}\right)-\mathcal{K}_{\Lambda}\left(m_{2} \mid n_{2}, n_{1}, n_{3}\right)-\mathcal{K}_{\Lambda}\left(m_{3} \mid n_{3}, n_{1}, n_{2}\right) \\
& +2 \mathcal{K}_{\Lambda}\left(0 \mid n_{1}, n_{2}, n_{3}\right),
\end{aligned}
$$

where

$$
\mathcal{K}_{\Lambda}\left(m \mid n_{1}, n_{2}, n_{3}\right)=\Lambda^{2} \int_{0}^{\infty} \mathrm{d} t \exp \left(-s_{m} t\right) f_{n_{1} n_{2} n_{3}}(t), \quad s_{m} \equiv \frac{m^{2}}{\Lambda^{2}},
$$

with

$$
\begin{aligned}
f_{n_{1} n_{2} n_{3}}(t)=\frac{1}{\left(t+n_{1}\right)^{2}}\{ & \ln \left(t+n_{1}+n_{3}\right)+\ln \left(t+n_{1}+n_{2}\right)+ \\
& \left.-\ln \left[t+\frac{n_{1} n_{2}+n_{1} n_{3}+n_{2} n_{3}}{n_{2}+n_{3}}\right]-\ln \left(n_{2}+n_{3}\right)\right\} .
\end{aligned}
$$


To find the required terms in the expansion of the Laplace transform (G.16) the Handelsman-Lew theorem [19] can be employed. It gives

$$
\mathcal{K}_{\Lambda}\left(m \mid n_{1}, n_{2}, n_{3}\right)=-\Lambda^{2} \mathcal{Q}\left(n_{1}, n_{2}, n_{3}\right)-\mathscr{L}_{\Lambda}\left(m \mid n_{2}, n_{3}\right)+\mathcal{O}\left(\Lambda^{0}\right),
$$

where

$$
\mathcal{Q}\left(n_{1}, n_{2}, n_{3}\right)=\left\{\sum_{l=1}^{3} \frac{1}{n_{l}}\right\} \ln \left\{\sum_{i=1}^{2} \sum_{j=i+1}^{3} n_{i} n_{j}\right\}-\sum_{i=1}^{2} \sum_{j=i+1}^{3}\left\{\frac{1}{n_{i}}+\frac{1}{n_{j}}\right\} \ln \left(n_{i}+n_{j}\right),
$$

and

$$
\mathscr{L}_{\Lambda}\left(m \mid n_{2}, n_{3}\right)=m^{2} \ln \frac{m^{2}}{\Lambda^{2}}\left\{\frac{1}{2} \ln \frac{m^{2}}{\Lambda^{2}}+\gamma_{E}-1+\ln \left(n_{2}+n_{3}\right)\right\} .
$$

Thus, the integral (G.11) has the form

$$
\begin{aligned}
I_{\Lambda}\left(m_{1}, m_{2}, m_{3} \mid\right. & \left.n_{1}, n_{2}, n_{3}\right)=\frac{1}{(4 \pi)^{4}}\left\{\Lambda^{2} \mathcal{Q}\left(n_{1}, n_{2}, n_{3}\right)+\right. \\
+ & \left.\mathscr{L}_{\Lambda}\left(m_{1} \mid n_{2}, n_{3}\right)+\mathscr{L}_{\Lambda}\left(m_{2} \mid n_{1}, n_{3}\right)+\mathscr{L}_{\Lambda}\left(m_{3} \mid n_{1}, n_{2}\right)\right\}+\mathcal{O}\left(\Lambda^{0}\right) .
\end{aligned}
$$

Note that the integral $I_{\Lambda}\left(0,0,0 \mid n_{1}, n_{2}, n_{3}\right)$ is infrared convergent and elementary (in the Schwinger parametrization):

$$
I_{\Lambda}\left(0,0,0 \mid n_{1}, n_{2}, n_{3}\right)=\frac{1}{(4 \pi)^{4}} \Lambda^{2} \mathcal{Q}\left(n_{1}, n_{2}, n_{3}\right) .
$$

Combining the above formula with the inequality

$$
\frac{e^{-(n-1) k_{E}^{2} / \Lambda^{2}}}{k_{E}^{2} e^{k_{E}^{2} / \Lambda^{2}}+m^{2}} \leq \frac{e^{-n k_{E}^{2} / \Lambda^{2}}}{k_{E}^{2}+m^{2}}+2 \frac{m^{2}}{\Lambda^{2}} \frac{e^{-n k_{E}^{2} / \Lambda^{2}}}{k_{E}^{2}},
$$

and monotonicity of the first term on the r.h.s. in $m$, it is easy to show that the difference between $I_{\Lambda}$ (G.11) and $I_{\Lambda}^{\text {tot }}$ (G.1) is a bounded function of $\Lambda$ for $\Lambda \rightarrow \infty$. Thus, in this limit,

$$
I_{\Lambda}^{\text {tot }}\left(m_{1}, m_{2}, m_{3} \mid n_{1}, n_{2}, n_{3}\right)=I_{\Lambda}\left(m_{1}, m_{2}, m_{3} \mid n_{1}, n_{2}, n_{3}\right)+\mathcal{O}\left(\Lambda^{0}\right) .
$$

The integral $\mathbb{J}_{\Lambda}=J_{\Lambda}^{\text {tot }}\left(m_{1}, m_{2} \mid n_{1}, n_{2}, n_{3}\right)$ defined in (G.2) can be conveniently calculated as a power series in $n_{3}$ :

$$
(4 \pi)^{4} \mathbb{J}_{\Lambda}=\sum_{s=0}^{\infty} \frac{\left(2 n_{3}\right)^{2 s}}{(2 s) !} \tilde{K}_{s} F_{s}\left(\alpha_{1}, m_{1}^{2}\right) F_{s}\left(\alpha_{2}, m_{2}^{2}\right), \quad \alpha_{i} \equiv n_{i}+n_{3},
$$

where

$$
\tilde{K}_{n}=\frac{\Gamma\left(n+\frac{1}{2}\right)}{\Gamma(n+2) \Gamma\left(\frac{1}{2}\right)},
$$

and

$$
F_{s}(\alpha, x) \equiv 2 \int_{0}^{\infty} \mathrm{d} k k^{3} \frac{k^{2 s}}{\Lambda^{2 s}} \frac{e^{-(\alpha-1) k^{2} / \Lambda^{2}}}{e^{k^{2} / \Lambda^{2}} k^{2}+x}=\sum_{n=0}^{\infty} x^{n} G_{n}(\alpha, s, x),
$$


with

$$
G_{n}(\alpha, s, x)=\int_{0}^{\infty} \mathrm{d} t \frac{t^{s+1}}{\Lambda^{2 s}} \frac{e^{-\alpha t / \Lambda^{2}}}{(t+x)^{n+1}}\left(1-e^{-t / \Lambda^{2}}\right)^{n} .
$$

Since $G_{0}=\mathcal{O}\left(\Lambda^{2}\right)$ and $G_{3}=\mathcal{O}\left(\Lambda^{-4}\right)$, it is easy to show that the series (G.24) can be replaced by the sum of its first three terms at most (the remaining terms give to the sum (G.22) contributions of order $\mathcal{O}\left(\Lambda^{-1}\right)$ ). It is also easy to determine with required accuracy the asymptotic, for $\Lambda \rightarrow \infty$, forms of $G_{0}, G_{1}$ and $G_{2}$ (the cases $s=0, s=1$ and $s \geq 2$ have to be considered separately). With these approximations the series (G.22) can be summed. Thus,

$$
\begin{aligned}
J_{\Lambda}^{\mathrm{tot}}\left(m_{1}, m_{2} \mid n_{1}, n_{2}, n_{3}\right)=\frac{1}{(4 \pi)^{4}} \frac{1}{2 !}\{ & \left\{\tilde{J}_{\Lambda}^{\mathrm{tot}}\left(m_{1}^{2}, m_{2}^{2} \mid n_{1}+n_{3}, n_{2}+n_{3}, n_{3}\right)+\right. \\
& \left.+\tilde{J}_{\Lambda}^{\mathrm{tot}}\left(m_{2}^{2}, m_{1}^{2} \mid n_{2}+n_{3}, n_{1}+n_{3}, n_{3}\right)\right\},
\end{aligned}
$$

where the divergent part of $\tilde{J}_{\Lambda}^{\text {tot }}$ is

$$
\begin{gathered}
\tilde{J}_{\Lambda}^{\text {tot }}\left(x_{1}, x_{2} \mid \alpha_{1}, \alpha_{2}, n_{3}\right)=-\frac{\Lambda^{4}}{n_{3}^{2}} \ln \left(1-\frac{n_{3}^{2}}{\alpha_{1} \alpha_{2}}\right)+\delta_{\Lambda}^{2} x_{1} x_{2}+ \\
+\delta_{\Lambda}\left\{\frac{n_{3}^{2} x_{1}^{2}}{\alpha_{2}^{2}}-\frac{2\left(\alpha_{1}+2\right) x_{1}^{2}}{\alpha_{2}}-2 x_{1} x_{2}\left[\ln \frac{1+\alpha_{1}}{2}+\ln \frac{x_{1}}{\mu^{2}}-1\right]\right\}+ \\
+2 \Lambda^{2} \frac{x_{1}}{\alpha_{2}}\left\{\ln \frac{x_{1}}{\mu^{2}}-\left(2+\delta_{\Lambda}+\ln 2\right)+\ln \left(1+\alpha_{1}-\frac{n_{3}^{2}}{\alpha_{2}}\right)+\right. \\
\left.-\alpha_{2} \frac{1+\alpha_{1}}{n_{3}^{2}} \ln \left(1-\frac{n_{3}^{2}}{\alpha_{2}+\alpha_{1} \alpha_{2}}\right)\right\}+\mathcal{O}\left(\Lambda^{0}\right) .
\end{gathered}
$$

(The dependence on $\mu$ is spurious - it cancels between logarithms and $\delta_{\Lambda}$ ). Terms of order $\mathcal{O}\left(\Lambda^{0}\right)$ can be determined in the same way (with the aid of expansion of the dilogarithm $\left.\mathrm{Li}_{2}(z)\right)$.

The remaining two integrals, (G.3) and (G.4), can be calculated in precisely the same way as $J_{\Lambda}^{\text {tot }}$ :

$$
\begin{aligned}
& N_{2, \Lambda}^{\mathrm{tot}}\left(m_{1}, m_{2} \mid n_{1}, n_{2}, n_{3}\right)=\frac{1}{(4 \pi)^{4}} \frac{1}{2 !}\left\{\tilde{N}_{2, \Lambda}^{\mathrm{tot}}\left(m_{1}^{2}, m_{2}^{2} \mid n_{1}+n_{3}, n_{2}+n_{3}, n_{3}\right)+\right. \\
& \left.+\tilde{N}_{2, \Lambda}^{\text {tot }}\left(m_{2}^{2}, m_{1}^{2} \mid n_{2}+n_{3}, n_{1}+n_{3}, n_{3}\right)\right\},
\end{aligned}
$$

where

$$
\begin{aligned}
& \tilde{N}_{2, \Lambda}^{\mathrm{tot}}\left(x_{1}, x_{2} \mid \alpha_{1}, \alpha_{2}, n_{3}\right)=\frac{\Lambda^{4}}{2 n_{3}^{4}}\left\{-3 n_{3}^{2}+\left(n_{3}^{2}-3 \alpha_{1} \alpha_{2}\right) \ln \left(1-\frac{n_{3}^{2}}{\alpha_{1} \alpha_{2}}\right)\right\}+ \\
& -\frac{1}{2} \delta_{\Lambda}\left\{x_{1}^{2} \frac{\alpha_{1}+2}{\alpha_{2}}-\frac{n_{3}^{2} x_{1}^{2}}{\alpha_{2}^{2}}+x_{1} x_{2}\left[\ln \frac{1+\alpha_{1}}{2}+\ln \frac{x_{1}}{\mu^{2}}-1\right]\right\}+\frac{1}{4} \delta_{\Lambda}^{2} x_{1} x_{2}+ \\
& +\Lambda^{2} \frac{x_{1}}{2 \alpha_{2}}\left\{\ln \frac{x_{1}}{\mu^{2}}-\left[3 \alpha_{2} \frac{1+\alpha_{1}}{n_{3}^{2}}+\delta_{\Lambda}+\ln 2+\frac{1}{2}\right]+\ln \left(1+\alpha_{1}-\frac{n_{3}^{2}}{\alpha_{2}}\right)+\right. \\
& \left.+\alpha_{2} \frac{1+\alpha_{1}}{n_{3}^{4}}\left(2 n_{3}^{2}-3 \alpha_{2}\left(1+\alpha_{1}\right)\right) \ln \left(1-\frac{n_{3}^{2}}{\alpha_{2}+\alpha_{1} \alpha_{2}}\right)\right\}+\mathcal{O}\left(\Lambda^{0}\right) .
\end{aligned}
$$


and

$$
M_{1, \Lambda}^{\mathrm{tot}}\left(m_{1}, m_{3} \mid n_{1}, n_{2}, n_{3}\right)=\frac{1}{(4 \pi)^{4}} \tilde{M}_{1, \Lambda}^{\mathrm{tot}}\left(m_{1}^{2}, m_{3}^{2} \mid n_{1}+n_{2}, n_{2}, n_{3}+n_{2}\right),
$$

where

$$
\begin{gathered}
\tilde{M}_{1, \Lambda}^{\text {tot }}\left(x_{1}, x_{3} \mid \alpha_{1}, n_{2}, \alpha_{3}\right)=-\frac{\Lambda^{4}}{\alpha_{1} n_{2}^{3}}\left\{n_{2}^{2}+\alpha_{1} \alpha_{3} \ln \left(1-\frac{n_{2}^{2}}{\alpha_{1} \alpha_{3}}\right)\right\}+ \\
+\delta_{\Lambda} \frac{n_{2}}{6 \alpha_{1}^{3} \alpha_{3}}\left\{3 x_{1}^{2} \alpha_{1}^{3}+3 x_{1} x_{3} \frac{\alpha_{1}^{3} \alpha_{3}}{1+\alpha_{1}}+x_{3}^{2} \alpha_{3}\left(2 n_{2}^{2}-3 \alpha_{1}\left(2+\alpha_{3}\right)\right)\right\}+ \\
+\frac{\Lambda^{2}}{4 n_{2}^{3} \alpha_{1}^{2}}\left\{2 x_{3} n_{2}^{4} \ln \frac{x_{3}}{\mu^{2}}-2 n_{2}^{2} \alpha_{1}\left(x_{3}+2 x_{1} \alpha_{1}+x_{3} \alpha_{3}\right)-n_{2}^{4} x_{3}\left(3+2 \delta_{\Lambda}+\ln 4\right)+\right. \\
+2 x_{3} n_{2}^{4} \ln \left(1+\alpha_{3}-\frac{n_{2}^{2}}{\alpha_{1}}\right)-2 x_{3} \alpha_{1}^{2}\left(1+\alpha_{3}\right)^{2} \ln \left(1-\frac{n_{2}^{2}}{\alpha_{1}+\alpha_{1} \alpha_{3}}\right)+ \\
\left.+4 x_{1} \alpha_{1}^{2}\left(n_{2}^{2}-\alpha_{3}\left(1+\alpha_{1}\right)\right) \ln \left(1-\frac{n_{2}^{2}}{\alpha_{3}+\alpha_{1} \alpha_{3}}\right)\right\}+\mathcal{O}\left(\Lambda^{0}\right) .
\end{gathered}
$$

Open Access. This article is distributed under the terms of the Creative Commons Attribution License (CC-BY 4.0), which permits any use, distribution and reproduction in any medium, provided the original author(s) and source are credited.

\section{References}

[1] G. 't Hooft and M.J.G. Veltman, Regularization and renormalization of gauge fields, Nucl. Phys. B 44 (1972) 189 [InSPIRE].

[2] P.H. Chankowski, A. Lewandowski, K.A. Meissner and H. Nicolai, Softly broken conformal symmetry and the stability of the electroweak scale, Mod. Phys. Lett. A 30 (2015) 1550006 [arXiv: 1404.0548] [INSPIRE].

[3] A. Latosinski, A. Lewandowski, K.A. Meissner and H. Nicolai, Conformal Standard Model with an extended scalar sector, JHEP 10 (2015) 170 [arXiv:1507.01755] [INSPIRE].

[4] G. Kleppe and R.P. Woodard, Nonlocal Yang-Mills, Nucl. Phys. B 388 (1992) 81 [hep-th/9203016] [INSPIRE].

[5] D. Evens, J.W. Moffat, G. Kleppe and R.P. Woodard, Nonlocal regularizations of gauge theories, Phys. Rev. D 43 (1991) 499 [InSPIRE].

[6] Y.-M.P. Lam, Perturbation Lagrangian theory for scalar fields: Ward-Takahasi identity and current algebra, Phys. Rev. D 6 (1972) 2145 [INSPIRE].

[7] Y.-M.P. Lam, Equivalence theorem on Bogolyubov-Parasiuk-Hepp-Zimmermann renormalized Lagrangian field theories, Phys. Rev. D 7 (1973) 2943 [INSPIRE].

[8] J.H. Lowenstein, Normal product quantization of currents in Lagrangian field theory, Phys. Rev. D 4 (1971) 2281 [inSPIRE].

[9] J.H. Lowenstein, Differential vertex operations in Lagrangian field theory, Commun. Math. Phys. 24 (1971) 1 [INSPIRE]. 
[10] C.P. Martin and D. Sánchez-Ruiz, Action principles, restoration of BRS symmetry and the renormalization group equation for chiral non-Abelian gauge theories in dimensional renormalization with a nonanticommuting $\gamma_{5}$, Nucl. Phys. B 572 (2000) 387 [hep-th/9905076] [INSPIRE].

[11] D.R.T. Jones, Comment on "bare Higgs mass at Planck scale", Phys. Rev. D 88 (2013) 098301 [arXiv: 1309.7335] [INSPIRE].

[12] M.S. Al-sarhi, I. Jack and D.R.T. Jones, Quadratic divergences in gauge theories, Z. Phys. C 55 (1992) 283 [INSPIRE].

[13] Y. Hamada, H. Kawai and K.-Y. Oda, Bare Higgs mass at Planck scale, Phys. Rev. D 87 (2013) 053009 [Erratum ibid. D 89 (2014) 059901] [arXiv: 1210.2538] [INSPIRE].

[14] D. Feldman, Z. Liu and P. Nath, The Stueckelberg $Z^{\prime}$ extension with kinetic mixing and milli-charged dark matter from the hidden sector, Phys. Rev. D 75 (2007) 115001 [hep-ph/0702123] [INSPIRE].

[15] C. Becchi, A. Rouet and R. Stora, Renormalization of the Abelian Higgs-Kibble model, Commun. Math. Phys. 42 (1975) 127 [INSPIRE].

[16] D. Binosi, J. Papavassiliou and A. Pilaftsis, Displacement operator formalism for renormalization and gauge dependence to all orders, Phys. Rev. D 71 (2005) 085007 [hep-ph/0501259] [InSPIRE].

[17] S. Weinberg, The quantum theory of fields, volume 1, Cambridge University Press, Cambridge U.K. (1995).

[18] M. Abramowitz and I.A. Stegun, Handbook of mathematical functions, U.S.A. (1972).

[19] R.A. Handelsman and J.S. Lew, Asymptotic expansion of Laplace transforms near the origin, SIAM J. Math. Anal. 1 (1970) 118.

[20] G. Bonneau, Some fundamental but elementary facts on renormalization and regularization: a critical review of the eighties, Int. J. Mod. Phys. A 5 (1990) 3831 [InSPIRE].

[21] O. Piguet and S.P. Sorella, Algebraic renormalization, Springer-Verlag, Berlin Heidelberg Germany (1995).

[22] J. Zinn-Justin, Renormalization of gauge theories, in Trends in elementary particle theory: international summer institute on theoretical physics in Bonn 1974, Lect. Notes Phys. 37 (1975) 1, Springer-Verlag, Berlin Germany (1975).

[23] W. Zimmermann, Normal products and the short distance expansion in the perturbation theory of renormalizable interactions, Annals Phys. 77 (1973) 570 [Lect. Notes Phys. 558 (2000) 278] [INSPIRE].

[24] G. Bandelloni, A. Blasi, C. Becchi and R. Collina, Nonsemisimple gauge models: 1. Classical theory and the properties of ghost states, Annales Poincaré Phys. Theor. 28 (1978) 225 [INSPIRE].

[25] G. Bandelloni, A. Blasi, C. Becchi and R. Collina, Nonsemisimple gauge models: 2. Renormalization, Annales Poincaré Phys. Theor. 28 (1978) 255 [InSPIRE].

[26] E. Kraus, Renormalization of the electroweak Standard Model to all orders, Annals Phys. 262 (1998) 155 [hep-th/9709154] [INSPIRE]. 
[27] W. Hollik, E. Kraus, M. Roth, C. Rupp, K. Sibold and D. Stöckinger, Renormalization of the minimal supersymmetric Standard Model, Nucl. Phys. B 639 (2002) 3 [hep-ph/0204350] [INSPIRE].

[28] P.A. Grassi, T. Hurth and M. Steinhauser, Practical algebraic renormalization, Annals Phys. 288 (2001) 197 [hep-ph/9907426] [INSPIRE].

[29] P.A. Grassi, T. Hurth and M. Steinhauser, The algebraic method, Nucl. Phys. B 610 (2001) 215 [hep-ph/0102005] [INSPIRE].

[30] P.A. Grassi and T. Hurth, On the two loop electroweak amplitude of the muon decay, hep-ph/0101183 [INSPIRE].

[31] P.A. Grassi, T. Hurth and M. Steinhauser, Noninvariant two loop counterterms for the three gauge boson vertices, JHEP 11 (2000) 037 [hep-ph/0011067] [INSPIRE].

[32] P. Breitenlohner and D. Maison, Dimensional renormalization and the action principle, Commun. Math. Phys. 52 (1977) 11 [inSPIRE].

[33] P. Breitenlohner and D. Maison, Dimensionally renormalized Green's functions for theories with massless particles. 1, Commun. Math. Phys. 52 (1977) 39 [INSPIRE].

[34] P. Breitenlohner and D. Maison, Dimensionally renormalized Green's functions for theories with massless particles. 2, Commun. Math. Phys. 52 (1977) 55 [INSPIRE].

[35] D. Sánchez-Ruiz, BRS symmetry restoration of chiral Abelian Higgs-Kibble theory in dimensional renormalization with a nonanticommuting $\gamma_{5}$, Phys. Rev. D 68 (2003) 025009 [hep-th/0209023] [INSPIRE].

[36] I. Fischer, W. Hollik, M. Roth and D. Stöckinger, Restoration of supersymmetric Slavnov-Taylor and Ward identities in presence of soft and spontaneous symmetry breaking, Phys. Rev. D 69 (2004) 015004 [hep-ph/0310191] [InSPIRE].

[37] C. Becchi, On the construction of renormalized gauge theories using renormalization group techniques, hep-th/9607188 [INSPIRE].

[38] M. Bonini, M. D'Attanasio and G. Marchesini, BRS symmetry for Yang-Mills theory with exact renormalization group, Nucl. Phys. B 437 (1995) 163 [hep-th/9410138] [INSPIRE].

[39] M. D'Attanasio and T.R. Morris, Gauge invariance, the quantum action principle and the renormalization group, Phys. Lett. B 378 (1996) 213 [hep-th/9602156] [INSPIRE].

[40] M. Bonini, M. D'Attanasio and G. Marchesini, BRS symmetry from renormalization group flow, Phys. Lett. B 346 (1995) 87 [hep-th/9412195] [INSPIRE].

[41] M. Bonini, M. D'Attanasio and G. Marchesini, Renormalization group flow for $\mathrm{SU}(2)$ Yang-Mills theory and gauge invariance, Nucl. Phys. B 421 (1994) 429 [hep-th/9312114] [INSPIRE].

[42] M.B. Fröb, J. Holland and S. Hollands, All-order bounds for correlation functions of gauge-invariant operators in Yang-Mills theory, arXiv:1511.09425 [INSPIRE].

[43] M.B. Fröb and J. Holland, All-order existence of and recursion relations for the operator product expansion in Yang-Mills theory, arXiv:1603.08012 [INSPIRE].

[44] G. Barnich and M. Henneaux, Renormalization of gauge invariant operators and anomalies in Yang-Mills theory, Phys. Rev. Lett. 72 (1994) 1588 [hep-th/9312206] [INSPIRE].

[45] P.A. Grassi, The Abelian anti-ghost equation for the Standard Model in the 't Hooft background gauge, Nucl. Phys. B 537 (1999) 527 [hep-th/9804013] [INSPIRE]. 
[46] Particle Data Group collaboration, K.A. Olive et al., Review of particle physics, Chin. Phys. C 38 (2014) 090001 [InSPIRE].

[47] C.-S. Chu, P.-M. Ho and B. Zumino, Non-Abelian anomalies and effective actions for a homogeneous space G/H, Nucl. Phys. B 475 (1996) 484 [hep-th/9602093] [INSPIRE].

[48] I. Jack and H. Osborn, General background field calculations with Fermion fields, Nucl. Phys. B 249 (1985) 472 [INSPIRE].

[49] M.E. Machacek and M.T. Vaughn, Two loop renormalization group equations in a general quantum field theory. 1. Wave function renormalization, Nucl. Phys. B 222 (1983) 83 [INSPIRE].

[50] M.E. Machacek and M.T. Vaughn, Two loop renormalization group equations in a general quantum field theory. 2. Yukawa couplings, Nucl. Phys. B 236 (1984) 221 [inSPIRE].

[51] M.E. Machacek and M.T. Vaughn, Two loop renormalization group equations in a general quantum field theory. 3. Scalar quartic couplings, Nucl. Phys. B 249 (1985) 70 [InSPIRE].

[52] P.H. Chankowski, Lectures on quantum field theory, unpublished.

[53] K. Fujikawa, Remark on the subtractive renormalization of quadratically divergent scalar mass, Phys. Rev. D 83 (2011) 105012 [arXiv:1104.3396] [inSPIRE].

[54] A.L. Kataev and K.V. Stepanyantz, Scheme independent consequence of the NSVZ relation for $N=1$ SQED with $N_{f}$ flavors, Phys. Lett. B 730 (2014) 184 [arXiv:1311.0589] [INSPIRE].

[55] S.S. Aleshin, I.O. Goriachuk, A.L. Kataev and K.V. Stepanyantz, The NSVZ scheme for $N=1 S Q E D$ with $N_{f}$ flavors, regularized by the dimensional reduction, in the three-loop approximation, arXiv:1610.08034 [INSPIRE].

[56] M.J.G. Veltman, The infrared-ultraviolet connection, Acta Phys. Polon. B 12 (1981) 437 [INSPIRE].

[57] P.H. Chankowski, S. Pokorski and J. Wagner, $Z^{\prime}$ and the Appelquist-Carrazzone decoupling, Eur. Phys. J. C 47 (2006) 187 [hep-ph/0601097] [INSPIRE].

[58] S.P. Martin, Two loop effective potential for a general renormalizable theory and softly broken supersymmetry, Phys. Rev. D 65 (2002) 116003 [hep-ph/0111209] [INSPIRE].

[59] K.A. Meissner and H. Nicolai, Conformal symmetry and the Standard Model, Phys. Lett. B 648 (2007) 312 [hep-th/0612165] [INSPIRE].

[60] K.A. Meissner and H. Nicolai, Neutrinos, axions and conformal symmetry, Eur. Phys. J. C 57 (2008) 493 [arXiv: 0803.2814] [INSPIRE].

[61] D. Buttazzo et al., Investigating the near-criticality of the Higgs boson, JHEP 12 (2013) 089 [arXiv: 1307.3536] [INSPIRE].

[62] A. Blasi, O. Piguet and S.P. Sorella, Landau gauge and finiteness, Nucl. Phys. B 356 (1991) 154 [INSPIRE]. 